\title{
Mandenkan
}

MANDENIKAN Bulletin semestriel d'études linguistiques mandé

$60 \mid 2018$

Numéro 60

\section{Dictionnaire mwan-français}

Mwan-French Dictionary

МУАН-ФРАНЦУЗСКИЙ СЛОВАРЬ

\section{Elena Perekhvalskaya et Moïse Yegbé}

\section{(2) OpenEdition}

Journals

Édition électronique

URL : https://journals.openedition.org/mandenkan/1873

DOI : 10.4000/mandenkan. 1873

ISSN : 2104-371X

Éditeur

Llacan UMR 8135 CNRS/Inalco

\section{Référence électronique}

Elena Perekhvalskaya et Moïse Yegbé, « Dictionnaire mwan-français », Mandenkan [En ligne], 60 | 2018, mis en ligne le 30 janvier 2019, consulté le 08 juillet 2021. URL : http://journals.openedition.org/ mandenkan/1873 ; DOI : https://doi.org/10.4000/mandenkan.1873

Ce document a été généré automatiquement le 8 juillet 2021.

\section{cc) (†) (-)}

Les contenus de Mandenkan sont mis à disposition selon les termes de la Licence Creative Commons Attribution - Pas d'Utilisation Commerciale - Partage dans les Mêmes Conditions 4.0 International. 


\title{
Dictionnaire mwan-français
}

\author{
Mwan-French Dictionary \\ МУАН-ФРАНЦУЗСКИЙ СЛОВАРЬ
}

Elena Perekhvalskaya et Moïse Yegbé

\section{NOTE DE L'ÉDITEUR}

Avec la collaboration de : Vincent Yegbé [contributeur]

\section{Avant-propos}

\section{Informations générales}

1 La langue mwan (hhbib lgcode : Mwa ; ISO 639-2 [moa]) appartient au groupe mandésud de la famille linguistique mandé. Les Mwan vivent dans 21 villages dans les souspréfectures de Kongasso et Kounahiri (région de Béré). Environ un tiers d'entre eux vit dans des centres urbains en dehors de la région, l'immigration interne des Mwan considérablement augmenté pendant la période de la guerre civile (2002-2011). Le mwan est parlé par plus de 20.000 personnes (Simons \& Fennig 2018).

2 La majorité des Mwan vivant dans les villages parlent aussi le gouro et le dioula qui sont des langues importantes et prestigieuses. Certains Mwan, sourtout des hommes, parlent aussi le français. L'occupation principale des Mwan est aujourd'hui la plantation de noix de cajou, et toutes les interactions commerciales se font en dioula ou en français.

3 Les données de ce dictionnaire ont été recueillies au cours de notre travail entre 2003 et 2018. Les auteurs tiennent à remercier les assistants de langue mwan, Antoine Yegbé, Too Yegbé, Amos Gogbé et de nombreux habitants des villages mwan (surtout de Bambalouma et Kongasso) pour leur aide inestimable et leur volonté de nous aider dans notre travail. 
Nous exprimons également nos remerciements à la branche ivoirienne de la Société Internationale de Linguistique, et tout particulièrement à Mme Margrit Bolli.

\section{Lettres et sons}

L'élément prosodique principal du mwan est le pied, unité d'un niveau supérieur à la syllabe, cf. (Vydrine $2005 ; 2010$ ). C'est l'unité prosodique qui peut se composer d'une ou deux syllabes qui se comportent phonétiquement comme une structure unique. Le pied en mwan est porteur du ton; il est caractérisé aussi par la nasalité / oralité et l'harmonie des voyelles (dans un pied, une voyelle semi-ouverte, / $\varepsilon /$ ou /o/, ne peut pas se combiner avec une voyelle semi-fermée, /e/ ou /o/). postérieures), l'aperture (fermées, mi-fermées, mi-ouvertes, ouvertes) et la nasalisation. Les voyelles postérieures sont arrondies.

Tableau 1. Les voyelles

\begin{tabular}{|l|l|l|l|l|l|}
\hline \multicolumn{2}{|l|}{ orales } & \multicolumn{2}{l|}{ nasalisées } \\
\hline & antérieure & postérieure & y & antérieure & postérieure \\
\hline fermée & i & u & & i & $\underset{\sim}{\text { u }}$ \\
\hline mi-fermée & e & o & & & \multirow{2}{*}{} \\
\hline mi-ouverte & E & J & & থ \\
\hline ouverte & a & & a & \\
\hline
\end{tabular}

11 2.2.2. Voyelles longues. Phonétiquement, toutes les voyelles sauf $\eta$ peuvent être brèves ou longues. Les voyelles longues sont considérées comme des combinaisons des voyelles identiques. Il y a des paires minimales : bo 'rester' - bo

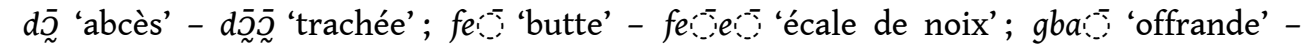
gbai:iai:' 'cadavre'.

12 Les voyelles longues sont désignées par les lettres redoublées.

13 2.2.3. Nasalisation. En mwan la nasalisation est un trait pertinent du pied entier, c.-àd. que dans un pied nasalisé, l'ensemble des voyelles et des consonnes sont nasalisées. À l'écrit, la nasalisation n'est marquée dans le pied qu'une seule fois. Il y a deux façons différentes pour marquer la nasalité vocalique : 1) par l'utilisation des lettres «m » et « $\mathrm{n}$ » pour les pieds nasaux avec les phonèmes / $\mathrm{b} / \mathrm{ou} / \mathrm{d} / ; 2$ ) par la lettre « $\mathrm{n}$ » après une voyelle : / $\mathrm{b}^{\bar{\varepsilon}} \mathrm{d} \overline{\tilde{c}} /$ 'serpent' est écrit $m l \varepsilon$, la nasalité du pied étant notée par la lettre

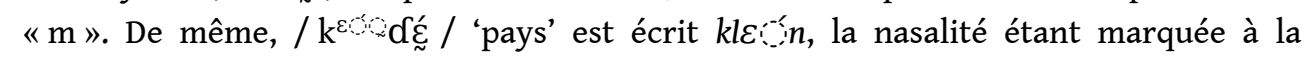
française par la lettre « $\mathrm{n}$ » après la voyelle correspondante. Dans la transcription 
scientifique, les voyelles nasalisées sont désignés par le signe «" au-desous de la lettre : a. position médiane dans un pied de types CVLV, CvLVy.

Tableau 2. Les consonnes

\begin{tabular}{|l|l|l|l|l|l|l|}
\hline & & labiale & apicale & palatale & vélaire & labio-vélaire \\
\hline \multirow{3}{*}{ occlusive } & non voisée & $\mathrm{p}$ & $\mathrm{t}$ & & $\mathrm{k}$ & $\mathrm{kp}$ \\
\cline { 2 - 8 } & voisée & $\mathrm{b}$ & $\mathrm{d}$ & & $\mathrm{g}$ & $\mathrm{gb}$ \\
\hline \multirow{3}{*}{ fricative } & non voisée & $\mathrm{f}$ & $\mathrm{s}$ & & & \\
\cline { 2 - 8 } & voisée & $\mathrm{v}$ & $\mathrm{z}$ & & & \\
\hline \multirow{2}{*}{ implosive et sonore } & & $\mathrm{6}[\mathrm{6}, \mathrm{m}]$ & $\mathrm{d}[\mathrm{l}, \mathrm{n}]$ & $\mathrm{j}[\mathrm{j}, \mathrm{n}]$ & & $\mathrm{w}[\mathrm{w}, \mathrm{w}]$ \\
\hline \multirow{2}{*}{ affriquée } & non voisée & & & $\mathrm{t}$ & & \\
\cline { 2 - 8 } & voisée & & & $\mathrm{d}$ & & \\
\hline
\end{tabular}

Les sonantes [m], [n], [n] et [w] n'ont pas de statut phonémique, étant les réalisations positionnelles des phonèmes non nasaux. Les réalisations nasales ou orales des phonèmes $/ \mathrm{d} /, / \mathrm{b} /, \mathrm{j} /$ et $/ \mathrm{w} /$ dépendent de la qualité nasale / orale du pied. Dans l'orthographe mwan l'allomorphe non-nasal de l'implosive / $\mathbb{C} /$ est représenté par la lettre « $1 »$.

\subsection{Les tons}

mwan comporte trois tons phonologiques de base : haut (H), moyen (M) et bas (B). Dans la transcription scientifique, les tons sont désignés par des signes diacritiques : le ton haut par l'accent aigu (v́); le ton moyen par le macron ( $\bar{v})$; le ton bas par l'accent grave (ì).

Exemples de triplets minimaux :

- kùe 'ratel', ku ie 'néré', kúe 'fardeau';

- kàà 'lime'; ka í 'poisson', káá 'gratter'.

Les pieds de types CVV, ClVV, CVy peuvent porter neuf combinaisons de tons : HH (v́v),

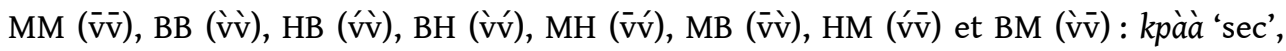
kpáa ' 'arbre (sp.)', kpáá 'hangar' ; tàa 'grand-père', ta ià 'ou (particule)', ta áa 'il faut' ; wE introduites : « 0 » et « $\varepsilon$ » pour les voyelles semi-ouvertes, et « $y$ » pour la voyelle nasale complètement fermée. 

«bh» ou la lettre « $m$ » selon l'oralité / nasalité du pied; l'implosive / $\mathfrak{d}$ / est marquée par « 1 » ou « $\mathrm{n}$ ». Les consonnes bifocals sont marquées par les digraphs « gb » et « $\mathrm{kp}$ ». Les consonnes palatales sont marquées comme suit : $/ \mathrm{t} / \mathrm{par}$ « $\mathrm{c} », / \mathrm{d} / \mathrm{par} \ll \mathrm{j} », \mathrm{j} / \mathrm{par}$ «y».

L'alphabet mwan :

a b bh cde $\varepsilon$ fggbijk kplm n yo o prstuvwyz

\subsubsection{Désignation des tons}

Les tons haut et bas sont marqués par des signes diacritiques, le ton moyen n'est pas marqué :

\begin{tabular}{|l|l|l|}
\hline ton haut & $\mathrm{H}$ & accent aigu (v́) \\
\hline ton moyen & $\mathrm{M}$ & $(\mathrm{v}) ;$ \\
\hline ton bas & $\mathrm{B}$ & accent grave (v). \\
\hline
\end{tabular}

\subsubsection{Règles de lecture : de la lettre au son}

Tableau 3. Lecture des lettres

\begin{tabular}{|c|c|c|c|}
\hline Lettre & Son & Exemple & Remarques \\
\hline A a & /a/ & ca / ţā/ 'dette' & \\
\hline $\mathrm{Bb}$ & $/ \mathrm{b} /$ & bie /biē/ 'éléphant' & \\
\hline $\mathrm{Bh}$ bh & /6/ & bhlele /Gđđēđē/ 'manger' & consonne implosive bilabiale \\
\hline $\mathrm{Cc}$ & $/ \mathrm{t} S /$ & càn / țà/ 'or' & affriquée palatale sourde \\
\hline D d & $/ \mathrm{d} /$ & do /dō/ 'un' & \\
\hline E e & /e/ & yele /jēōè/ 'voir' & \\
\hline$\varepsilon \varepsilon$ & $/ \varepsilon /$ & 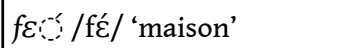 & voyelle ouverte antérieure \\
\hline F f & /f/ & fle $\varepsilon^{\prime}$ /fớć / 'peau' & \\
\hline Gg & /g/ & gaan /gāā/ 'pied' & \\
\hline Gb gb & $/ g b /$ & gbaan /gbāāa / 'babouin' & labio-vélaire bifocale sonore \\
\hline I i & /i/ & yi /ji/ 'eau' & \\
\hline $\mathrm{Jj}$ & $/ d+3 /$ & jiè /dżiè/ 'grand-mère' & affriquée palatale sonore \\
\hline K k & $/ \mathrm{k} /$ & kaa /kāā/ 'poisson' & \\
\hline Kp kp & $/ \mathrm{kp} /$ & kpéé /kpéé/ 'ventre' & labio-vélaire bifocale sourde \\
\hline L 1 & $/ \mathrm{d} /$ & lo:le /đòdè/ 'acheter' & $\begin{array}{l}\text { consonne implosive alvéolaire / sonante } \\
\text { lateral }\end{array}$ \\
\hline $\mathrm{M} \mathrm{m}$ & $/ \mathrm{m} /$ & $m l \varepsilon / \complement^{\bar{\varepsilon}} \mathrm{d} \overline{\tilde{d}} /$ serpent & sonante nasale \\
\hline $\mathrm{N} \mathrm{n}$ & $/ \mathrm{d} / \mathrm{n} /$ & $n \varepsilon \varepsilon / \mathcal{d} \bar{c} \bar{c} \bar{c} /$ 'mère' & $\begin{array}{l}\text { consonne implosive alvéolaire / sonante } \\
\text { nasale }\end{array}$ \\
\hline
\end{tabular}




\begin{tabular}{|c|c|c|c|}
\hline$y_{y}$ & $/ y /$ & $\dot{y} / \hat{y} /$ 'moi' & \\
\hline Oo & /o/ & koo /kōō/ 'dos' & \\
\hline ว כ & /ว/ & go : /gò/ 'chimpanzé' & voyelle ouverte postérieure \\
\hline $\mathrm{Pp}$ & $/ \mathrm{p} /$ & po-inle /póddē/ 'percer' & \\
\hline $\mathrm{R} \mathrm{r}$ & $/ \mathrm{r} /$ & rájó /rádzó/ 'radio' & dans les mots empruntés \\
\hline $\mathrm{Ss}$ & /s/ & sàà /sàà/ 'natte' & \\
\hline $\mathrm{Tt}$ & $/ \mathrm{t} /$ & $t \varepsilon^{-i n n} / \mathrm{t} \mathcal{c} /$ 'rouge' & \\
\hline $\mathrm{Uu}$ & $/ \mathrm{u} /$ & $d u / d \bar{u} /$ 'buffle' & \\
\hline $\mathrm{Vv}$ & $/ \mathrm{v} /$ & 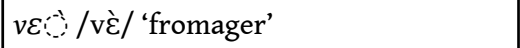 & \\
\hline $\mathrm{W} w$ & $\begin{array}{l}/ \mathrm{w} /, \quad / \\
\text { w/ }\end{array}$ & 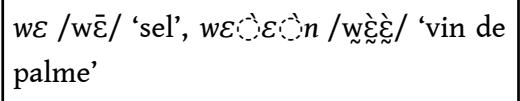 & sonante sonante nasale \\
\hline Y y & $/ \mathrm{j} /, / \mathrm{n} /$ & 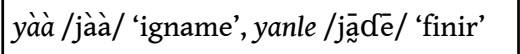 & sonante sonante nasale \\
\hline $\mathrm{Zz}$ & /z/ & $z i / \mathrm{z}$ / 'chemin' & \\
\hline
\end{tabular}

2.6. L'orthographe précédente. L'alphabet créé par la Société Internationale de Linguistique (Orthographe 2000) a été utilisée dans plusieures publications mwan, et surtout dans la traduction du Nouveau Testament (2011).

La nécessité du changement d'orthographe a pour le but de trouver une solution aux problèmes suivants.

Dans l'orthographe 2000, le marquage des tons des mots dissyllabiques était ambigu. Si les deux voyelles portent le même ton, seul le ton de la première voyelle était marqué : 'peni /pénîi/ 'dard'; bie /bīē/ 'éléphant'; -vako /vàkò/ 'canne à sucre'. Si le ton de la première voyelle est bas et le ton de la seconde est «plus haut» (H ou M), le mot est suivi d'une apostrophe:-gbaan'/gbàa / 'chien'; -soo' /soó/ 'cheval'. Si le ton de la première voyelle est haut, et le ton de la deuxième est "plus bas » (B ou $M)$, la fin du mot est marquée par un trait d'union : 'pubJ- /púbj̄/ 'saluer' ; 'kpata- /kpátà/ 'claie'.

Pour les mots de trois ou plus de trois syllabes seul le ton de la voyelle initiale est noté : -amasroyi /àmāasròyí/ 'parce que'; laanima /lāānî́mā// 'en haut'; ' 'pkena /ýkènà / 'bonjour'.

Tableau 4. Phonèmes et leur représentation orthographique

\begin{tabular}{|c|c|c|c|c|}
\hline Son & Lettre & Exemple & $\begin{array}{l}\text { orthographe } \\
2000\end{array}$ & $\begin{array}{l}\text { orthographe } \\
2009\end{array}$ \\
\hline \multicolumn{5}{|c|}{ Voyelles } \\
\hline /a/ & a & $/$ ţā/ 'dette' / sàà/ 'natte & $\mathrm{ca}$-saa & ca sàà \\
\hline$/ \varepsilon /$ & $\varepsilon \varepsilon \varepsilon$ & 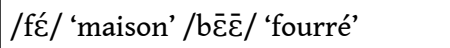 & 'f $\varepsilon$ bec & 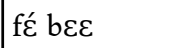 \\
\hline /e/ & e ee & /jēe/ 'voir /Gēē/ 'piège' & ye bhee & ye bhee \\
\hline /i/ & i ii & /ji/ 'eau /sîii/ ‘clan' & -yi ‘sii & yì sîí \\
\hline /o/ & ว วว & /gò/ 'chimpanzé' /gbòj̀/ 'ancêtre' & -go -gbวo & gò gbòગ̀ \\
\hline
\end{tabular}




\begin{tabular}{|c|c|c|c|c|}
\hline /o/ & o oo & /tó/ 'laisser / kōō/ 'dos & 'to koo & tó koo \\
\hline$/ \mathrm{u} /$ & u uu & /dī/ 'taureau' /fúú/ 'sueur' & du 'fuu & du fúú \\
\hline /a a / & $\mathrm{m}, \mathrm{n}+\mathrm{a}, \mathrm{an}$ & 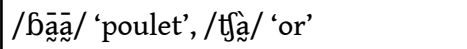 & maa-can & maa càn \\
\hline$/ \varepsilon /$ & $\mathrm{m}, \mathrm{n}+\varepsilon, \varepsilon \mathrm{n}$ & 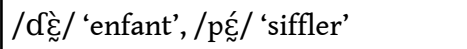 & $-n \varepsilon ' p \varepsilon n$ & nè pén \\
\hline$/ \mathrm{i} /$ & $m, n+i$, in & /6̧ĩi/ 'moi (Emph.)' /gbĩ / 'chasser' & 'mi -gbin & mí gbìn \\
\hline /כ2/ & $m, n+0$, on & 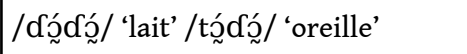 & 'nono 'tron & nró trón \\
\hline$/ \mathrm{u} /$ & $m, n+u, u n$ & $\begin{array}{l}\text { /Gū } / \text { marqueur de pluriel /kú / } \\
\text { 'attraper' }\end{array}$ & mu 'kun & mu kún \\
\hline$/ y /$ & $y$ & $/ y ́$ / ‘je, moi’ & 'y & $\dot{y}$ \\
\hline \multicolumn{5}{|c|}{ Consonnes } \\
\hline /6/ & {$[6]-\mathrm{bh}[\mathrm{m}]-\mathrm{m}$} & /6lē/ 'manger' /61్ㅠ/ 'serpent' & bhle mle & bhle mle \\
\hline$/ \mathrm{d} /$ & $\begin{array}{l}{[\mathrm{d} / \mathrm{l}]-1[\mathrm{r}]-\mathrm{r}[\mathrm{n}]} \\
-\mathrm{n}\end{array}$ & 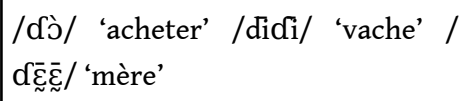 & -lo dri nec & 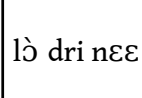 \\
\hline$/ \mathrm{j} /$ & $y$ & /jàà/ 'igname' /nāa / 'finir' & -yaa yan & yàà yan \\
\hline /w/ & w & 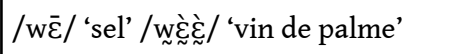 & we $-w \varepsilon \varepsilon n$ & we wèc̀n \\
\hline$/ \mathrm{p} /$ & $\mathrm{p}$ & /pó / 'percer' & 'pon & pón \\
\hline$/ \mathrm{b} /$ & $b$ & /biēé/'éléphant' & bie & bie \\
\hline /f/ & $\mathrm{f}$ & /f'́d'́́/ 'peau' & 'fle & flé \\
\hline /v/ & $\mathrm{v}$ & /vغ̀/ 'fromager' & $-\mathrm{v} \varepsilon$ & vغे \\
\hline$/ \mathrm{t} /$ & $t$ & $/ \mathrm{t} \varepsilon_{\alpha}^{\prime} /$ 'rouge' & 'ten & tén \\
\hline$/ d /$ & $d$ & /dō/ 'un' & do & do \\
\hline /s/ & $\mathrm{s}$ & /sàà/ 'natte' & -saa & sàà \\
\hline /z/ & $\mathrm{z}$ & /zì/ 'chemin' & $\mathrm{zi}$ & zi \\
\hline$/ \mathrm{k} /$ & $\mathrm{k}$ & /kứ/ 'attraper' & -kun & kún \\
\hline /g/ & $g$ & /gāāa / 'pied' & gaan & gaan \\
\hline $\begin{array}{l}\text { / } \\
\mathrm{kp} /\end{array}$ & $k p$ & /kpéé/ 'ventre' & 'kpee & kpéé \\
\hline $\begin{array}{l}\text { / } \\
\mathrm{gb} /\end{array}$ & $g b$ & /gbāāa / 'babouin' & gbaan & gbaan \\
\hline$/ \mathrm{tg} /$ & c & $/ \mathrm{t}$ à $/$ 'or' & -can & càn \\
\hline $\begin{array}{l}/ \\
\mathrm{d} 3 /\end{array}$ & $j$ & /dżiè/ 'grand-mère' & -jie & jiè \\
\hline
\end{tabular}

\subsubsection{Exemples de texte écrit dans les différents systèmes orthographiques}

- Transcription phonologique :

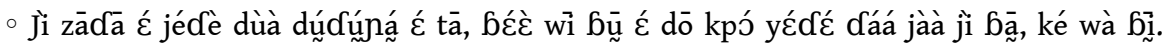

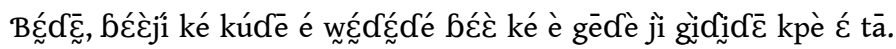


- L'orthographe 2009 :

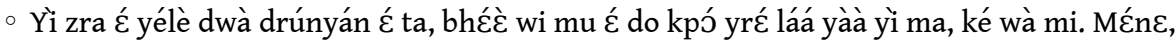
bhéčyí ké Kúle, é wléćnlé bhéc̀ ké è gelè yì glinle kpè ć ta.

- L'orthographe 2000 :

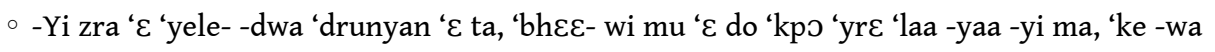

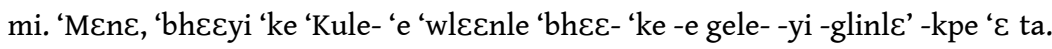

- Traduction :

- C'était la sécheresse partout dans le monde et tous les animaux ne pouvaient pas trouver d'eau à boire. Ma chère, c'est ainsi que Tortoise s'est levée et est allée chercher de l'eau dans la brousse.

\section{Composition du dictionnaire}

L'objectif de ce Dictionnaire est d'inclure toutes les couches du vocabulaire de la langue mwan, y compris les néologismes, les emprunts ainsi que les différents registres stylistiques. Sont exclus du Dictionnaire les mots de circonstance, y compris des emprunts occasionnels (surtout $\mathrm{du}$ français) que les citadins, surtout éduqués, emploient couramment, mais qui ne sont pas encore bien enracinés dans la langue mwan.

\subsection{Sources}

Nous avons utilisé les publications en mwan, surtout les livres éducatifs de la SIL, et les ouvrages linguistiques suivants: les dissertations de Caroline Fleming (1995) et d'Antoine Yegbé (2000) ; l'article sur la phonologie mwan de Margrit Bolli et Eva Flik (1978). La majeure partie de l'information a été recueillie au cours du travail avec des assistants de langue en 2003-2018. Des aspects différents de la grammaire mwan sont décrits dans les publications de l'un des auteurs (Perekhvalskaya 2004; 2007; 2010; $2011 ; 2014 ; 2016 ; 2017)$. Dans le travail sur ce dictionnaire, les auteurs se sont inspirés par les autres dictionnaires des langues mandé-sud, en particulier, (Vydrine \& Kességbeu 2008).

\subsection{Formes dérivées et composées}

Les formes dérivées sont présentées comme des entrées séparées. Les dérivés réguliers (ceux dont le sémantisme est en correspondance directe avec le sémantisme des composants, sans qu'un sens supplémentaire y soit rajouté) ne sont pas indiqués. Il s'agit surtout des formes dérivées par les suffixes et les suffixoïdes comme :

- $\mathbf{n} \mathbf{E}^{-1}$ - à valeur diminutif (de $n \varepsilon^{-} ;$'enfant')

- le - féminin (des êtres humains ; de le 'femme')

- da - féminin (des animaux ; de da 'femelle')

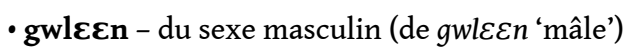

\subsection{Marques des parties de discours (catégories grammaticales)}

Les homonymes lexico-grammaticaux sont donnés sous des entrées différentes :

- lìi $n$ 1. $r n$ (contr) bouche $\diamond$ yintìi narine ; yonlíi mamelle 2. porte $\diamond f \mathcal{E}^{-}$ maison

- lìi pstp (contr) 1. en, dans 2. sur (juste une partie de l'objet est en contact avec la surface)

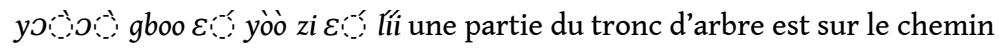

Marques des parties du discours en mwan :

- adj-adjectif 


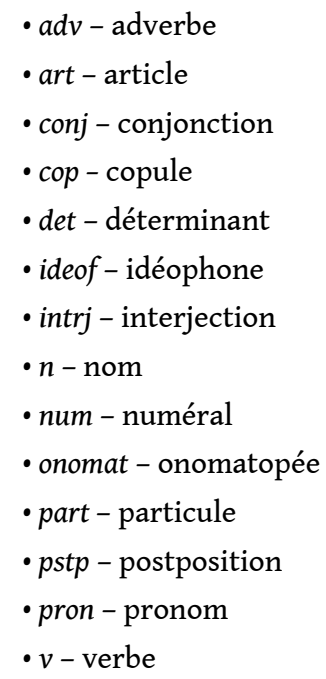

3.3.2. Les verbes sont distingués par rapport à la présence ou l'absence du complément d'objet direct :

- vi-verbe intransitif ;

- $v t$ - verbe transitif (le critère formel de la transitivité est la présence du complément d'objet direct devant le verbe) ;

- $v r$ - verbe réfléchi (verbe dont le sujet et le complément d'objet direct coïncident).

Les autres valences des verbes sont présentées de façon explicite autant que possible: la postposition qui marque souvent la fonction d'un complément d'objet indirect est placée entre parenthèses et précédée d'une préposition française qui en indique le sens approximatif, par ex. :

- pele vt... dire (à - ni)

Si l'équivalent français comporte une préposition qui n'est pas mise entre parenthèses, cela veut dire que l'argument introduit par la préposition française correspond en mwan au complément d'objet direct, par ex. :

- lé.jále $v p$... 3. vi rencontrer (qui - lé)

3.3.3. Pour les noms, deux marqueurs sont utilisés pour indiquer les types de valence différents :

- $r n$ - «nom relationnel»: substantif qui entre dans une construction génitive-possessive comme second élément sans être précédé par la marque possessive $a$, par ex. : le gbe 'la main de la femme' - cf. «nom libre » le a dunc'; 'le vêtement de la femme'.

- $n$ - «nom libre» : substantif autosémantique. Dans le dictionnaire, tout nom qui n'est pas marqué comme rn est a priori un «nom libre».

3.3.4. Pour les pronoms personnels, la série est indiquée (cf. Tableau 6). Là où des pronoms de séries différentes ont la même forme, ils sont présentés dans la même entrée, par exemple :

-ý pron 1. je (1 pers. sg., la série sujette de base) ; 2. me (1 pers. sg., la série non-sujette)

Les formes contractées des pronoms sont données dans la même entrée, quoique formées de différentes composantes.

Pour les pronoms français, seules les formes masculines sont indiquées. Ainsi, dans l'entrée suivante, il correspond à il, elle :

- è pron il (3 pers. sg. ; série sujet de base) 
3.4. Le sémantisme du mot polysémique (et de chaque homonyme lexicogrammatical) est donné par sens, dont chacun est marqué par un chiffre arabe.

-gbe $n$ 1. $r n$ bras, patte avant (d'animal, d'insecte), aile (d'oiseau)... 2. branche (d'arbre)... 3. côté... 4. manière, façon

51 3.4.1. Là où cela est nécessaire, l'équivalent français peut être précisé par un commentaire entre parenthèses et en italiques (une sémantisation). Chaque sens du mot mwan est traduit par un équivalent français ou par plusieurs synonymes. Si un mot n'a pas d'équivalent français, son sens est interprété par un commentaire en italiques.

- moso $\mathrm{n}$ n boule magique (médicament traditionnel : des petites boules de couleur noir ou brun foncé)

Si l'équivalent français lui-même a plus d'un sens ou si sa correspondance au sens du mot mwan n'est qu'approximative, il peut être accompagné de commentaires spécifiants (des sémantisations) qui sont mis entre parenthèses et en italiques.

- nòó $r n$ trompe (d'éléphant)

3.4.2. Le "sens» peut avoir aussi les subdivisions suivantes: marque d'usage, [commentaires ethnographiques et encyclopédiques]; (commentaires grammaticaux).

• matón <ma+tón> adj 1. aigre, tourné, gâté (des aliments)... 2. Fig. mécontent, qui a l'air mécontent (de personnes)

- so $n$ 1. tissu $\diamond$ sotànmi tisserand [traditionnellement, parmi les Mwans il n'y pas de tisserands, le tissage est généralement fait par les Dioula]

- ómoo pron 1. nous (1 pers. pl. excl., série emphatique)

3.4.3. Les unités phraséologiques et phrases plus ou moins figées sont précédées du signe de diamant $\Delta$

- màyà $n$ lutte (sportive) $\diamond$ màyà dánle lutter

Les exemples de l'usage sont précédés par le signe « $\square$ : l'exemple mwan est donné en italique ; la traduction française est donnée en caractères réguliers :

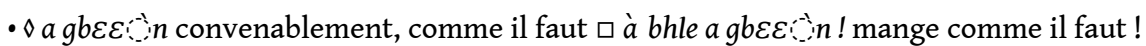

3.4.4. Les synonymes sont notés Syn. :

- lezip $\varepsilon<$ le+zi+p $\varepsilon>\mathrm{n}$ dot (les biens donnés selon le contrat de mariage pour l'épouse)... Syn. flú

57 3.4.5. Les noms de faune et de flore sont accompagnés des noms latins en italiques (à l'exception de ceux qui n'ont pas encore été identifiés) et d'un commentaire entre parenthèses. Le terme français est indiqué si disponible.

- sáa n 1. Alestes marcolepitodus aleste (poisson)

\subsection{Traduction}

Les sens des mots polysémiques sont présentés, selon l'histoire dérivative, en hiérarchie. La place du sens particulier dans cette hiérarchie est codée par des chiffres arabes.

Si les verbes diffèrent par leur transitivité, les sens sont indiqués par des chiffres arabes suivis de parenthèse : 1), 2) etc.

- siánle $v(m o b)$ 1) vr éternuer... 2) $v t$ faire éternuer 
61 3.5.2. Les expressions idiomatiques et les exemples servent à illustrer l'usage des mots. Les commentaires grammaticaux en italiques sont donnés si le mot est caractérisé par des irrégularités de forme ou d'usage.

Les unités idiomatiques sont disposées conformément à leur complexité et à leur sémantisme : les syntagmes attributifs sont donnés en premier lieu; puis suivent les locutions verbales.

\begin{tabular}{|c|c|c|c|c|c|c|}
\hline (1) & Záki & ò & go:ili & $n a ́-z i-i$ & |é & $n i$ \\
\hline & Jacques & Cop & argent & donner-Prog & $2 S g$ & Dat \\
\hline
\end{tabular}

\subsection{Caractéristiques stylistiques}

\subsubsection{La liste des marques de style et d'usage :}

- Fig. - figuré ; concernant les croyances traditionnelles des Mwan);

- Vulg. - vulgaire.

\section{Structure de la langue}

\subsection{Une caractéristique typologique}

Ex. :

Tous les registres du vocabulaire mwan sont inclus dans le dictionnaire. Le but est de fournir à chaque mot et expression mwan un équivalent français le plus proche possible par son sémantisme et ses caractéristiques stylistiques. Uniquement pour le vocabulaire des niveaux les plus bas (vocabulaire vulgaire et obscène), nous avons eu recours à des traductions françaises du registre neutre. Tous les mots et expressions qui ne sont pas de style neutre sont indiqués par les marques appropriées. Si la marque précède l'équivalent français, elle se rapporte au mot mwan; si elle suit l'équivalent français, elle est indicative des caractéristiques stylistiques de l'équivalent français.

- Animist. - mot/sens courant dans le langage du milieu animiste ;

-Arch. - archaïque (mot, sens ou emploi de l'ancienne langue, incompréhensible ou peu compréhensible de nos jours et employé pour obtenir un effet stylistique) ;

- Afr. - français africain (mot, sens ou emploi caractéristique du français africain) ;

- Christ. - mot/sens courant dans le langage du milieu chrétien ;

- Iv. - français ivoirien (mot, sens ou emploi caractéristique de l'usage ivoirien du français) ;

- Trad. - mot/sens courant dans le langage du milieu traditionnel (il s'agit surtout des termes

Le mwan est une langue isolante avec des éléments du synthétisme. Par le type d'expression des valeurs grammaticales, c'est une langue analytique; les formes synthétiques sont caractéristiques des pronoms et du verbe.

Les morphèmes dérivatifs et flexionnels sont agglutinants, il y a des cas de fusion.

4.2. L'ordre des mots est très strict. Il suit le modèle suivant : S (aux) od V Oi

1 L'ordre des mots est stable et grammaticalement significatif; toutes les positions syntaxiques obligatoires doivent être remplies, et aucune omission d'éléments n'est admise. 


\section{Tableau 5. Pronoms de base : personnel, démonstratif, anaphorique}

\begin{tabular}{|c|c|c|c|c|c|c|c|}
\hline & \multicolumn{3}{|c|}{ Singulier } & \multicolumn{4}{|c|}{ Pluriel } \\
\hline & 1 & 2 & 3 & $\begin{array}{l}1 \\
\text { excl }\end{array}$ & $1 \mathrm{incl}$ & 2 & 3 \\
\hline Sujet & \multirow{3}{*}{$\dot{y}$} & \multirow{3}{*}{ é } & $\grave{e}$ & \multirow{3}{*}{ ó } & \multirow{3}{*}{$k 0$} & \multirow{3}{*}{ ká } & \multirow{3}{*}{$\grave{o}$} \\
\hline Non-Sujet & & & $\grave{a}$ & & & & \\
\hline Réfléchi & & & é & & & & \\
\hline Emphatique & mí & $6 i$ & yéé & ómว̄ว̄ & 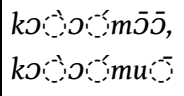 & $\begin{array}{l}\text { kámōj, } \quad \text { ká } \\
m u i_{\bar{j}}\end{array}$ & $\begin{array}{l}\text { wó } \bar{i} \\
(m u)\end{array}$ \\
\hline Impératif & - & 0 & - & - & - & $k \grave{a}$ & - \\
\hline $\begin{array}{l}\text { Contrastif } \\
\text { Démonstratif }\end{array}$ & - & - & $6 \varepsilon^{\prime-3}$ & - & - & - & $m u ́$ \\
\hline Anaphorique & - & - & $a ́$ & - & - & - & - \\
\hline
\end{tabular}

La distinction entre les formes sujette et non-sujette est manifestée uniquement pour les formes de $3 \mathrm{Sg}$ (les pronoms de base, réfléchi et anaphoriques). Les autres personnes utilisent les mêmes formes sans tenir compte du rôle syntaxique du participant codé.

5.1.2. Formes fusionnées. Les pronoms personnels forment des unités fusionnées avec certains mots fonctionnels (voir Tableau 6). Toutes les formes ne sont pas tout à fait morphologisées; la plupart d'entre elles peuvent être défusionnées lorsqu'elles sont prononcées lentement.

Tableau 6. Pronoms personnels fusionnés

\begin{tabular}{|c|c|c|c|c|c|c|c|c|}
\hline & \multirow{2}{*}{$\begin{array}{l}\text { Élément } \\
\text { fusionné }\end{array}$} & \multicolumn{3}{|c|}{ Singulier } & \multicolumn{4}{|c|}{ Pluriel } \\
\hline & & 1 & 2 & 3 & 1 excl & $1 \mathrm{incl}$ & 2 & 3 \\
\hline Fusionné avec la copule & ò & ò & ýóò & yóò & wóo & kJטים & káò & wòò \\
\hline Négatif & la & la & náá & yáá & wáá & $\begin{array}{l}\text { kJá } \\
\text { láa }\end{array}$ & $\begin{array}{l}\text { ká } \\
\text { láá }\end{array}$ & wa \\
\hline Négatif avec copule & la & náá ò & yáò & ya & wáo & ka & $\begin{array}{l}\text { ká } \\
\text { láo }\end{array}$ & waio \\
\hline
\end{tabular}




\begin{tabular}{|c|c|c|c|c|c|c|c|c|}
\hline $\begin{array}{l}\text { Négatif avec copule et } \\
\text { pronom non sujet } 3 S G\end{array}$ & la $a_{\text {ò }}$ & náà & yáa & $y a \hat{a}$ & wáa & $\begin{array}{l}k 00 \\
\text { làa }\end{array}$ & ká & $w a$ \\
\hline Possessif & $a-\bar{i}$ & $a$ & $y a ́$ & $y a$ & wáa & 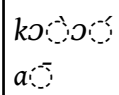 & $\begin{array}{l}k a ́ \\
a \bar{c}\end{array}$ & wa \\
\hline
\end{tabular}

5.1.3. Les pronoms focalisés sont formés par la contraction des pronoms de base avec les marqueurs de focus lè (sujet) et là (non-sujet).

Tableau 7. Pronoms focalisés (personnel, démonstratif et interrogatif)

\begin{tabular}{|c|c|c|c|c|}
\hline & \multicolumn{2}{|l|}{ Singulier } & \multicolumn{2}{|c|}{ Pluriel } \\
\hline & sujet & non-sujet & sujet & non-sujet \\
\hline Pronoms personnels (3 personne) & yélè & yélà & wóle & wólà \\
\hline Contrastifs démonstratifs & besile, $6 \varepsilon^{-1}$ & $6 \varepsilon^{\prime}-i l a ̀$ & múlè & múlà \\
\hline Pronoms interrogatifs dè 'qui' ; $m \varepsilon^{\prime-j}$ 'que' & dèle $m \varepsilon$ Silè & dèlà $m \varepsilon$ ì̀ & - & - \\
\hline
\end{tabular}

5.1.4. Pronoms portemanteau sujet-objet (voir Tableau 8). Certaines combinaisons sont prévisibles, mais pas toutes. Certaines de ces formes sont grammaticalisées (dans le tableau elles sont présentées en gras); dans les autres cas, la prononciation séparée est également acceptable.

\begin{tabular}{|c|c|c|c|c|c|c|c|}
\hline $\begin{array}{l}\text { Objet } \rightarrow \\
\text { Sujet } \downarrow\end{array}$ & & lé & $\grave{a}$ & & 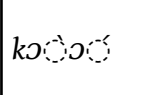 & ká & ò \\
\hline$\dot{y}$ & ý & gééé & $a_{z}$ & ǵó & ǵ k k & ǵ ká & ǵo \\
\hline é & yén & yéé & $y a ́$ & yéó & é ko & é ká & yéo \\
\hline$\grave{e}$ & yèn & yèé & $y \grave{a}$ & yòó & è k- & è ká & yòo \\
\hline ó & wón & óé & wá & wóó & 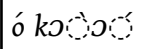 & ó ká & wóo \\
\hline או- & 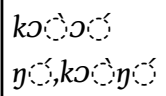 & $\begin{array}{l}k 00 \\
e\end{array}$ & $k \partial: a ́$ & 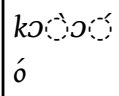 & אَ- & $\begin{array}{l}\text { kJ' } \\
\text { ká }\end{array}$ & $\begin{array}{l}k 0 \\
\text { o }\end{array}$ \\
\hline$k a ́$ & káń & káé & $k a ́ a ́$ & káó & ká ka & ká ká & káo \\
\hline ò & wòn & wò é & $w \grave{a}$ & wò ó & 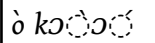 & ò ká & wò̀ \\
\hline
\end{tabular}

\section{Tableau 8. Pronoms portemanteau}

\section{5.2. Nom}

\subsubsection{Constructions génitives-possessives :}

-1) GN + a + GN (deux noms sont reliés par la postposition a i).

-2) GN + GN (deux noms se suivent directement).

La première construction est utilisée avec des noms autosémantiques en deuxième position ; la seconde est utilisée avec les noms relationnels.

\subsubsection{Constructions d'attribut nominal}


Dans les constructions d'attribut nominal le nom dépendant est préposé au nom principal et lui sert de modificateur (2b).

Une construction génitive-possessive :

• (2a) tùàbù a sroàsé 'soldat d'un Blanc'.

Une construction d'attribut nominal :

- (2b) tùàbù sroàsé 'soldat blanc'.

\subsubsection{Structure du groupe nominale}

Tableau 9. Structure du groupe nominale

\begin{tabular}{|c|c|c|c|c|c|c|c|}
\hline \multicolumn{2}{|c|}{$\begin{array}{l}\text { modificateur de pré- } \\
\text { noyau }\end{array}$} & \multicolumn{2}{|l|}{ Noyau } & \multicolumn{4}{|c|}{ Périphérie } \\
\hline modificateur & $a$ & $\begin{array}{l}\text { GN } \\
\text { principal }\end{array}$ & ATTR & $\begin{array}{l}\text { PL } \\
\text { NUM }\end{array}$ & $\begin{array}{l}\text { kpletrick } \\
\text { kpo té }\end{array}$ & $\begin{array}{l}\text { lé } \quad \text { ART } \\
\text { FOC REL }\end{array}$ & $\begin{array}{l}\text { bèbe } \\
k p \varepsilon a k e\end{array}$ \\
\hline-2 & $\mid-1$ & 0 & +1 & +2 & +3 & +4 & +5 \\
\hline
\end{tabular}

5.2.3.1. Le Noyau lexical d'un groupe nominal peut être constitué d'un groupe nominal (simple ou complexe) et d'une épithète suivant le groupe nominal.

5.2.3.2. La position +2 (nombre) peut être occupée par le marqueur de pluriel $m \bar{u}$ ou par un numeral cardinal.

5.2.3.3. La position +3 (spécificateurs de nombre) n'est présente que lorsque la position 2 est occupée par un numeral cardinal. Les spécificateurs des numeraux sont : kpo 'seulement, juste', kple 'n 'seulement (un)', té 'exactement, précisément'.

5.2.3.4. La position +4 (statut pragmatique) est occupée par les éléments suivants : démonstratif $l \in \varepsilon^{\prime}$ 'ceci' ; article $\varepsilon$; ; marqueurs de focus lè (sujet) et là (non-sujet) ; relativisateur lá 'lequel'.

5.2.3.5. La position +5 est occupée par des quantificateurs : bèbè 'beaucoup', sā 'peu', $k p \varepsilon^{--i n}$ 'tout', ké 'plusieurs'.

\begin{tabular}{|c|c|c|c|c|c|c|c|c|c|}
\hline (3) & 0 & Gbàgbo io & $a$ & màfá & glo: & $m u$ & $\varepsilon$ & kpe & síc-la. \\
\hline & $3 \mathrm{PL}$ & Gbagbo & POSS & fusil & dur & PL & ART & tout & casser-PRF \\
\hline & & -2 & -1 & 0 & +1 & +2 & +4 & +5 & \\
\hline
\end{tabular}

\subsection{Verbe}

5.3.1. Tout verbe, sauf dans la construction du 2 Sg Impératif, doit être précédé d'un sujet.

Les verbes transitifs doivent avoir le complément d'objet direct ouvertement exprimé, à la limite, par un élément fictif (pronom non-Sujet 3Sg à, les mots $p \bar{\varepsilon}$ 'chose', $m \bar{\varepsilon} \bar{\varepsilon}$ 'personne' etc.).

Les verbes réfléchis ont le complément d'objet direct exprimé par un pronom personnel correspondant au sujet ou par le pronom réfléchi é pour les formes de $3 \mathrm{Sg}$. 


\subsubsection{Formes verbales non-finies :}

\begin{tabular}{|l|l|}
\hline Nom verbal & V-lei \\
\hline Supin & V-lē \\
\hline Converbe conjonctif & V-lé $/ l e$ \\
\hline
\end{tabular}

\subsubsection{Constructions prédicatives :}

\begin{tabular}{|l|l|}
\hline habituel & S od $\bar{V}$ (ton moyen) \\
\hline perfectif & S od Và (forme perfective) \\
\hline progressif & S ò od V-zí (forme progressive) \\
\hline futur & S nui od $\mathrm{V}-l \bar{\varepsilon}$ \\
\hline résultatif & Od V-le ì ò \\
\hline impératif & Od V \\
\hline
\end{tabular}

Tableau 10. Conjugaison du verbe à $l \grave{\text { ' }}$ 'acheter'

\begin{tabular}{|c|c|c|c|c|}
\hline & \multicolumn{2}{|c|}{ Forme affirmative } & \multicolumn{2}{|l|}{ Forme négative } \\
\hline & $2 \mathrm{Sg}$ & $3 \mathrm{Sg}$ & $2 \mathrm{Sg}$ & $3 \mathrm{Sg}$ \\
\hline Habituel & yá & yà lō & yáá & ya: \\
\hline Perfectif & yá lúà & yà lùà & yáá lơ: & ya \\
\hline Progressif & yá lo zisión & yà lo: zí & yáa lo zí & ya $a$ \\
\hline Futur & é nư & è nu & yáá nu à lo & ya \\
\hline Resultatif & é lo:ilè ò & à loulè ò & é lo & à loule la \\
\hline Imperatif & àlo & - & 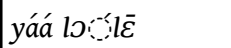 & - \\
\hline
\end{tabular}

5.3.5. Modèles de modifications tonales

Les mots mwans affichent trois types de changements du ton selon le ton du mot précédent, s'ils sont liés syntaxiquement.

-1) Modèle constant : le ton du mot ne change pas.

-2) Modèle contrastif :

\begin{tabular}{|l|l|}
\hline Ton du mot précédent & Ton du mot suivant \\
\hline Haut & Haut \\
\hline Moyen & lexical \\
\hline
\end{tabular}




\begin{tabular}{|l|l|}
\hline Bas & Haut \\
\hline
\end{tabular}

-3) Modèle coordinatif :

\begin{tabular}{|l|l|}
\hline Ton du mot précédent & Ton du mot suivant \\
\hline Haut & Tombant \\
\hline Moyen & lexical \\
\hline Bas & Bas \\
\hline
\end{tabular}

(mob). Les verbes du paradigme constant ne modifient leur ton lexical, des verbes du paradigme mobile changent leur ton selon le ton du mot précédent par des règles complexes (les modèles contrastif et coordonné sont utilisés en différentes formes aspecto-temporels).

- klìgle $v(m o b)$ 1) vi 1. se coincer...

- zinàle $v$ (const) 1$)$ vi descendre...

\section{Abréviations}

\begin{tabular}{|l|l|}
\hline 1 pers. & première personne \\
\hline adj & adjectif \\
\hline adv & adverbe \\
\hline Afr. & français africain \\
\hline Animist. & mot/sens courant dans le langage du milieu animiste \\
\hline Arch. & archaïque \\
\hline ART & l'article défini \\
\hline aux & mot auxiliaire \\
\hline
\end{tabular}




\begin{tabular}{|c|c|}
\hline B & ton bas \\
\hline const & verbe du paradigme constant \\
\hline Christ. & mot/sens courant dans le langage du milieu chrétien \\
\hline conj & conjonction \\
\hline cop & copule \\
\hline Dat & postposition à valeur d'adressée \\
\hline det & déterminant \\
\hline excl. & exclusif \\
\hline Fig. & figuré \\
\hline FOC & focalisateur \\
\hline FUT & futur \\
\hline GER & nom verbal \\
\hline $\mathrm{H}$ & ton haut \\
\hline ideof & idéophone \\
\hline incl. & inclusif \\
\hline intrj & interjection \\
\hline IRR & irréel \\
\hline Iv. & français ivoirien \\
\hline mob & verbe du paradigme mobile \\
\hline M & ton moyen \\
\hline $\mathrm{n}$ & nom \\
\hline NEG & négation \\
\hline num & numéral \\
\hline od & complément d'objet direct \\
\hline Oi & complément d'objet indirect \\
\hline onomat & onomatopée \\
\hline OPT & optatif \\
\hline part & particule \\
\hline PERF & perfectif \\
\hline pl. & pluriel \\
\hline POSS & marqueur possessif \\
\hline PRF & perfectif \\
\hline PROG & progressif \\
\hline pron & pronom \\
\hline pstp & postposition \\
\hline
\end{tabular}




\begin{tabular}{|l|l|}
\hline REL & marqueur relatif \\
\hline rn & nom relationnel \\
\hline S & sujet \\
\hline sg. & singulier \\
\hline Trad. & mot/sens courant dans le langage du milieu traditionnel \\
\hline V, v & verbe \\
\hline vi & verbe intransitif \\
\hline vr & verbe réfléchi \\
\hline vt & verbe transitif \\
\hline Vulg. & vulgaire \\
\hline
\end{tabular}

\section{Références} Yulia Mazurova, Andrej Kibrik \& Elena Markus (eds.), Jazyki mira: Jazyki mande (языки МИРА: ЯЗЫКИ МАНДЕ) [Languages of the world: Mande languages], 718-765. St. Petersburg: Nestor-Historia.

115 Simons, Gary F. \& Charles D. Fennig (eds.). 2018. Ethnologue: Languages of the World. $21^{\text {st }}$ ed. Dallas, Texas: SIL International. https://www.ethnologue.com/ (4 December, 2018).

116 Vydrine, Valentin. 2005. Quelques recommendations méthodologiques concernant la description des langues mandé-sud. Mandenkan 41. 1-22. 
117 Vydrine, Valentin. 2010. Le pied métrique dans les langues mandé. In Franck Floricic (ed.), Essais de typologie et de linguistique générale. Mélanges offerts à Denis Creissels, 53-62. Lyon: ENS Éditions. http://halshs.archives-ouvertes.fr/halshs-00715537. Vydrine, Valentin \& Mongnan Alphonse Kességbeu. 2008. Dictionnaire Dan-Français (dan de l'Est) avec une esquisse de grammaire du dan de l'Est et un index français-dan. St. Petersburg: Nestor-Istoria. http://halshs.archives-ouvertes.fr/halshs-00715560. Yegbé, Koffi Antoine. 2000. Processes of nominalization in Mwan. Nairobi: Nairobi Evangelical Graduate School of Theology Masters thesis.

\section{Dictionnaire mwan-français}

\section{$A-a$, comme a, á, à, à ż̀ć lè}

a conj marqueur de la possession $\square$ kòó a gba notre champ

á pron série contastive focalisée le, lui (3 pers. sg., en position de complément d'objet direct ou indirect)

à pron série non-sujet 1. le, la (3 pers. sg., en position de complément d'objet direct ou indirect) $\square$ ò nwà à gliple ils sont venus le regarder 2 . son (le possesseur avec un nom en relation) $\square$ è jà à gbe púbole tùàbù tré ta il est allé visiter son fils en ville $\diamond$ à bhغ̀c̀ké autre

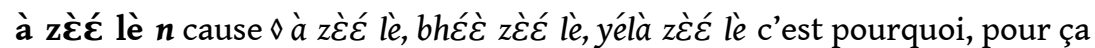

aa part particule emphatique $\square$ è bhé cíc ć kpén gwà bhé ma pléypléy aa elle l'a plumée entièrement!

áà, áá, á part est-ce que, n'est-ce pas? (marque de question rhétorique ou de précision) jóo à pelè bhí ni áa ? est-ce que c'est à toi que je dois le raconter ? $\square$ Zàle maa ć gwàn áá ? Zalé a vendu le poulet, non?

àànni intrj oui, si (réponse affirmative à une question négative ou affirmative) $\square$ yáá pcbhlele

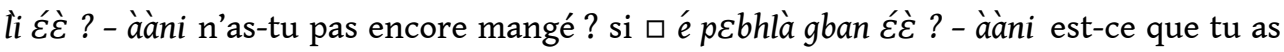
déjà mangé ? oui

ah intrj ah! (soupir de souffrance, douleur, fatigue)

áli conj même si

áliké conj même si, bien que, malgré (souvent avec la particule oo); $\square$ áliké la ò banzí oo, nغ̀ $m u$ ò $f \mathcal{E}$ toflà $\varepsilon$ ba bien qu'il pleuve, les enfants se baignent dans le marigot

àlùkú $n$ danse (de l'ethnie bété)

àma $a d v$ absolument, exclusivement $\square$ è blàán pon là bhlà àma il n'a mangé que du foutou banane $\square$ Zàle yáláà Màni kóó àma Zalé dois épouser Mani absolument (la dot est déjà payée)

àmasròyi <Dioula k'a măsذ̀ro> conj parce que, car $\square$ waa mí yé le àmasròyí mí mí magàànnà ils ne m'ont pas vu parce que je m’étais caché

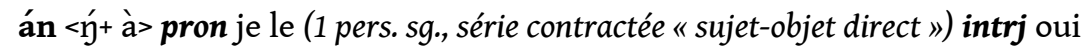

án $<\grave{y}+a>$ pron mon (1 pers. sg., série possessive)

Aoobààd $\varepsilon$ intrj bonjour, bonsoir (réponse d'une femme à la salutation d'un homme)

Aoobàànc̀ intrj bonjour, bonsoir (la réponse d'une femme à la salutation d'une femme) 


\section{$B-b$, comme ba, bàà, bààle}

ba $n$ 1. dioula (ethnie, langue) $\square$ ba mu a fétí yi le tabasakîi lé La Tabaski est la fête des Dioulas 2. musulman

ba $n$ 1. espace à l'intérieur de qqch 2. domicile, demeure (d'une personne) $\square$ Zinì ba lre c'est joli chez Jules 3. période de temps $\square$ voo bla ba laa so ý ni je n'aime pas la saison de l'harmattan

ba pstp 1. dans, à l'intérieur (dans un liquide, un feu, ou un milieu homogène) $\square$ yì ba dans l'eau $\square \grave{y} d \varepsilon$ sukalo klàa yaa gáfe yí $\varepsilon$ ba mon père a mis du sucre dans son café $\square$ kaa ò baá ć ba il y a du poisson dans le riz préparé 2. parmi $\square$ gbăádúá ple yàa maa mu ć ba il y avait deux canards parmi les poulets 3. chez, avec $\square$ kòó ba mwa ta chez nous les

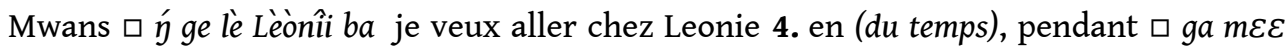
kun voo bla ba les gens tombent malade pendant l'harmattan $\diamond$ wáátí kpén ba toujours

bà $\boldsymbol{r n}$ poche (d'un vêtement ou d'un sac) [dans le vêtement traditionnel, souvent devant, sur le ventre] $\square$ gòli ò é bà yí l'argent est dans ta poche

bàà $n$ habillement, parure

bàá $\boldsymbol{n}$ riz (préparé) [est considéré comme le repas préféré pour un visiteur respectable, en particulier servi avec du poulet] $\square$ ý báá bhle tó lé je mange le riz avec la cuillère $\diamond$ bááyí baka (bouillie de riz avec du sucre) $\square$ ò bááyí mi zr Jan là zi ébèbèlé c'est le matin qu'on a l'habitude de consommer le baka; gbànglàn bàá purée de banane plantain mélangée avec de l'arachide grillée et pilée

bàá $\boldsymbol{r n}$ venin $\nabla m l \varepsilon$ bàá venin de serpent

bààle v 1) vt habiller, parer, saper $\square$ mí ý lú bààlà dunÈ dre ć lé j’ai bien habillé ma fille avec une nouvelle robe 2) $\operatorname{vr} \mathbf{1}$. se parer, se vêtir $\square$ mí ý bààla je me suis paré $\nabla$ bàa gbrya manière de s'habiller, vêtements $\square$ ba mu bàa gbeya laa ò mùa mu bàa gbcya gbec̀n les Dioulas s'habillent autrement que les Mwans; bàa $p \varepsilon$ parure 2. se moquer (de - ta) $\square$

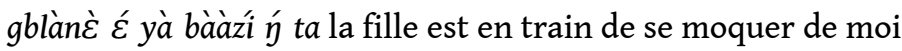

baán $\boldsymbol{r n}$ supplément, ajout, augmentation $\square$ ga é baán ò klázíi à ba sa maladie s'aggrave

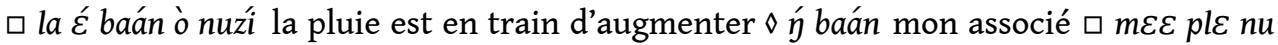
nulc, bhéc $m u k l \varepsilon$ ý baán lé deux personnes vont venir et ils se joindront à moi

bààp $\varepsilon n$ vêtement élégant, toilette de fête, parure $\square$ ké sró tán yi bhwà ò bààpe bèbè lo quand le jour de danse arrive, on achète beaucoup de toilettes élégantes

báfù $<$ Fr. baffle $>\boldsymbol{n}$ tambour (espèce) [droit en forme, souvent bilatéral, sa carcasse peut être de bois ou de métal, son son est plus bas que le son des autres tambours; il est consideré comme un instrument moderne]

baglinle <ba+glinle> vt observer, examiner $\square$ o kàkáó baglin pé póli ta bhéc̀ wà lo à zan kj̀j̀ on examine d'abord le cacao au port et on l'achète chez son propriétaire

bagole <ba+gole> $\boldsymbol{v t}$ trier $\square$ ó wáa kaa mu ć bago yi goon é ta nous trions nos poissons sur la rive de la rivière 2 . choisir $\square$ le yaga lá gè ò bagole la ǵ ta il est difficile pour moi de choisir parmi ces trois filles 
bajanwole $<$ ba+jan+wole $>\boldsymbol{v t}$ parler du comportement de qqn (avec un peu de critique) $\square \grave{e}$ mí là bajanwozi c'est de moi qu'il parle $\square$ Màní à drwànè Táátoo bajanwozí à de ni Mani discute le comportement de son neveu Tato avec son père

bakánle <ba+kánle> vt éclairer (rendre compréhensible) yá jàn ć bakán! éclaire ta parole ! sois lucide dans ta parole ! 2. s'éclaircir $\square$ yrEma ć bakààn le ciel s'est éclairci $\nabla a ̀$ yré bakààn il a quitté l'étape de bébé

bákì $<\mathrm{Fr}$. bac $>\boldsymbol{n}$ baccalauréat $\diamond$ báki yele avoir le baccalauréat

balàle, baale $v \mathbf{1}$ ) vi tomber $\diamond$ balàle ò être couché (après être tombé) $\square$ magròo balàle ò le manguier est tombé 2) vt 1. laisser tomber $\square$ ǵ tásá bàláá j'ai laissé (fait) tomber la

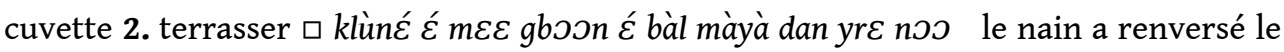
géant dans la lutte

bàlí $<$ Fr. baril $>\boldsymbol{n}$ baril

bàli $n$ victoire

bàlíkón $<$ Fr. baril+kóy $>\boldsymbol{n}$ baril

bàlò $<$ Dioula $>\boldsymbol{n}$ causerie, bavardage $\diamond$ bàlo klele bavarder, causer

bàlò̀ $<$ Fr. ballon $>\boldsymbol{n}$ ballon

bàloỳzonmi <bàlò̀+zon+mi> $\boldsymbol{n}$ joueur de football $\square$ ý dì mi ع́ gbe ò bàloỳzonmi gbènc̀ lé le fils de mon voisin est un grand joueur de football

bami $<$ ba+mi> $\boldsymbol{n}$ 1. Dioula (représentant de cette ethnie) 2. musulman $\square$ yaa yàà zi bhii è kle bami lé il ne voulait pas devenir musulman

bándí $<$ Fr. bandit> $\boldsymbol{n}$ bandit

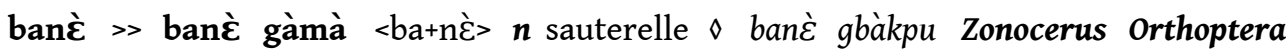
sauterelle (parasite des champs) $\square$ Syn. gàmà

banle $v$ 1. vi pleuvoir $\square$ yòo bhla zi la ban ébèbè lé pendant la saison des pluies il pleut beaucoup 2. se jeter (sur - ta) $\square$ sroónè bààn moo ta le chat s'est jeté sur la souris $\square$ ké ò pebhle é dùláá, yáá banle à ta, ká kpén a pe le kéle quand on dépose la nourriture, ne te jette pas dessus, elle appartient à tous

bàylí $n$ Argemona mexicana argémone, figuier infernal, pavot épineux

baya <ba+ya> $\boldsymbol{n}$ 1. traditions des Dioula 2. Islam [les Dioula sont des musulmans

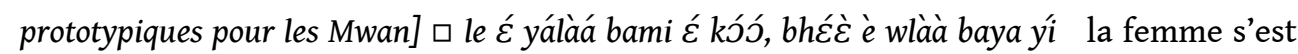
mariée avec un Dioula et elle est devenue musulmane

bèbè adj nombreux $\square$ gò do laa yàa lè, gò bèbè lè yàa ce n’était pas un seul chimpanzé, il y avait beaucoup de chimpanzés $\square$ ò kààlon gon ébèbè lé Bíjàn plé mu yí on vend beaucoup de crabes sur les marchés d'Abidjan

bébé $\boldsymbol{n}$ moucheron (petit, qui irrite avec son bourdonnement)

bénî $\boldsymbol{n}$ canne

beý $\boldsymbol{n}$ grenier

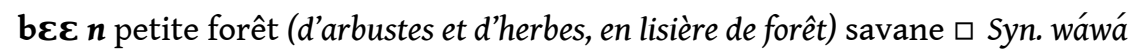

bè̀े $\boldsymbol{r n}$ poitrine (de personne, d'animal) $\diamond$ bè $\dot{\varepsilon}$ dele se frapper la poitrine (se vantant de bravoure)

béźgbé $\boldsymbol{n}$ Arch. tissu (industriel), coupon (de tissu)

béćgoli biviàn $n$ Perodicticus potto potto $\square$ Syn. twà 

mille francs beaucoup de fleurs pas le casser) petites souris

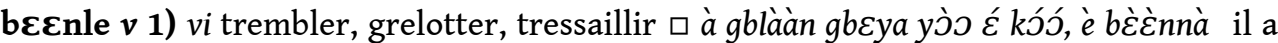

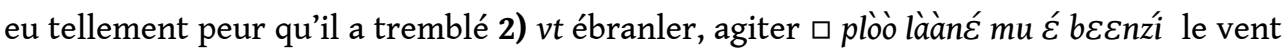
fait trembler les feuilles

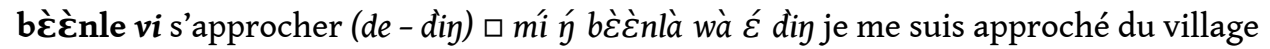

béli $<$ Fr. beurre> $\boldsymbol{n}$ beurre

bènwóle < ?+wole> $\boldsymbol{v} 1$. vi arriver à temps 2. s'accorder, se mettre d'accord (sur - ta) $\square$ wáa pléś gon jan é wóle blaan, ó bèn wòa après la discussion concernant notre commerce, nous nous sommes mis d'accord $\square$ ó pegée pólisié mu é bènwòà gòli wáádo gbawole là ta les policiers et moi, nous sommes tombés d'accord sur l'amende de cinq

bì $\boldsymbol{n}$ fleur [traditionnellement les fleurs n'ont pas de signification symbolique] $\diamond$ bi klele, bi kánle être en fleur, fleurir $\square$ lec là kwlécn bì kààn ébèbè lé cette année le coton a donné

bì $\boldsymbol{n}$ nuit (quand il fait sombre: de 19 à 5 heures) $\diamond$ bipóle faire (de la nuit), s'anuiter, être surpris par la nuit $\square m \varepsilon \varepsilon m u$ ć bi pwà gba la les gens étaient surpris par la nuit au champ ; bì pwà la nuit est tombée (il fait nuit); bi lá zan bhe la nuit suivante ; bì kpén zi toute la nuit ; bi dodo zi chaque nuit

bi part 1. comme (condition irréelle) ם ké mí lè bi léćmi lé, ý yáà nu gole lèc̀ya é yí si j'étais le président, j'aurais la tête à l'envers 2. marqueur de contrafactif $\square$ Zàan á gbú

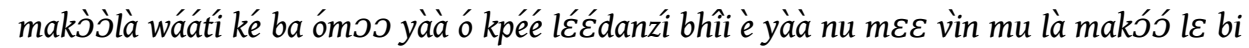
Jean s'est défendu lui-même, alors que nous pensions qu'il défendrait plutôt les autres

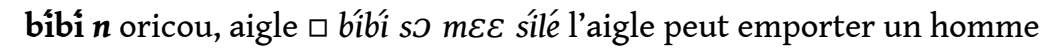

bie bèlì wó yrì <calque du dioula sàmá bèrì $\boldsymbol{n}$ arbre (esp. : si souple que l'éléphant ne peut

bie, biye $n$ Loxodonta africana éléphant $\square$ bie nè enfant d'éléphant $\diamond$ bie són ivoire ; bie zikùy les fesses d'éléphant (de qqn qui n'a pas de fesses)

bielenغ̀, biele $\boldsymbol{n}$ souris (très petite) [est considérée mangeable; on les prépare en brochettes sur le feu ou dans les marmites; les enfants les chassent en creusant leurs trous, d'abord on fait du feu devant le trou et on souffle sur le feu pour que la fumée les étouffe] 口 ó vòtàné É jà tépléy ta bhéc è bielenc̀ bèbè dià notre cadet est allé à la chasse et il a tué beaucoup de

biì part est-ce que (marque de question avec une nuance de doute) $\square$ ý yáa wà la wááti lá ýi Záàn jàn lá wóa é bhé yáán go bii ? est-ce que tu sais ce que Jean a dit quand j'étais au village ? व mèle klà yée Yrié láá nule, è rúsi mu ć núle é máà bii pourquoi Irié n'est pas venu, n'a-t-il pas appris l'arrivée des Russes?

biile $v(m o b)$ 1) vt 1. tordre, tourner $\square$ nè $\varepsilon$ yè án wla é lé bìilà l'enfant a courbé le bout de mon couteau $\square$ sЈ É bii é zi bhéc̀ ká lòmmli ć kan mets le pagne autour de tes reins et et allez cueillir les oranges 2 . tourner, tordre $\square$ maa $\varepsilon$ bhló bii! tords le cou du poulet $\square$ yà gbe biilà il a tordu le bras 3. bander, enrouler 4. tourner (cheval, vélo) $\square$ ǵ sòo $\varepsilon$ bhlo biilà gbe kòo ta j'ai tourné le vélo à gauche 2) vi 1. se courber, être courbé $\square$ án wlà $\varepsilon$ bìilà mon couteau s'est tordu 2. tourner, tordre, se rouler $\square$ zi É yòo biizíi le chemin tourne $\square$ à koo biile ò son dos est tordu $\diamond a ̀$ kpée bii son estomac tourne (fait mal)

bíkì $<$ Fr. bic $>\boldsymbol{n}$ stylo (à bille) 
binàle $v \boldsymbol{t}$ (const) soulever (des choses lourdes, des parties du corps) $\square$ nè É fúu láá sつ yrì gboo é binàle ta l'enfant est incapable de soulever le tronc d'arbre $\square$ mí ý gaan bina bhéc̀ án kpa tàbàli ć ta bhéċ wòn gaan ma bhe ć tékpa je soulève mon pied, je le mets sur la table pour qu'on soigne la plaie qui est desus

bijle $\boldsymbol{v t}$ (const) enterrer (personne, animal) $\diamond m \varepsilon \varepsilon b i y y r \varepsilon$ cimetière $\square$ mùà ta ò yàà $m \varepsilon \varepsilon m u$ biy f́́ zànta autrefois chez les Mwans on enterrait les gens derrière les maisons

bísì <Fr. (auto)bus> $\boldsymbol{n}$ autobus $\square m \varepsilon \varepsilon$ kpÉn bo bísi yí Bijian à Abidjan tout le monde prend le bus

bla $n$ 1. course $\square$ bla É yààn la course est terminée $\square$ ví̀̀n $\varepsilon$ si bla lè ké bhe kan maanc̀ ta l'avion est plus rapide que l'oiseau $\diamond$ blasanle courir, fuir $\square$ mle blasààn le serpent s'est évadé 2. vitesse

blà $n$ plante épineuse, "faux bambou». [les fibres qui sont extraites de ses feuilles sont utilisées pour faire les jupes des masques mwan] b blà bùu fibre de «faux bambou» $\square$ ò gbàgba diní kle blà bùu lé la jupe du masque est faite de fibres de «faux bambou »; blà yí vin de «faux bambou »

\section{blalè, blaalè $a d v$ tout de suite, rapidement}

blaale vi grossir, être en forme $\square$ le blaale lè so mwan gwláan mu ni les hommes mwan aiment les femmes en forme

blaale vt éplucher (fruit, légume) $\square$ súglùú blaa ! épluche le manioc !

blaan $r n$ 1. trace, empreinte $\diamond \grave{a}$ gaan blaan ses traces de pas ; à gbe blaan son écriture, la main de; blaan gò̀̀nle imiter la manière (des personnes, animaux) 2. blaan ta conséquence $\square$ à blaan ta nu klele à ma éyj̀o lé les conséquences seront mauvaises pour lui (lit. sur lui)

blàán, blàànná $\boldsymbol{n}$ banane (surtout plantain) $\diamond$ blaán bhe do une banane; blàán diin bananier, régime de bananes; blàán vlòj̀ main de bananes; tùàbú blàán banane (douce); blaán gba plantation de banane; blaán kplooo banane bouillie; blaán yáále chips de banane ; bláán pon foutou de banane

blaan, blan pstp 1. après $\square$ gwle blaan pebhle pléć klâ après la guerre la nourriture est devenue plus chère 2. sans $\square$ náá so yewole bhí blaan je ne peux pas travailler sans toi $\diamond$ bhé blaan à part ça

blàángbakpámi <blàan+gba+kpá+mi> $\boldsymbol{n}$ cultivateur de bananes

bláká $<$ Dioula $>\boldsymbol{n}$ force, santé

blàko $\boldsymbol{n}$ tige de «faux bambou » [s'utilise pour la fabrication des chaises, des lits et des

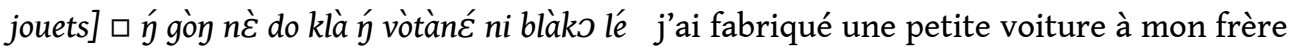
cadet avec du « faux bambou »

blay $\boldsymbol{n}$ caleçon traditionnel (mâle) [pièce d'étoffe que l'on porte drapée autour des hanches et qui couvre le sexe des hommes]

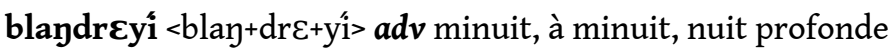

blasanle $<$ bla+sanle $>\boldsymbol{v}$ courir

bléy, bénî $n$ bâton, canne 口 yrèwimu ć tááwo béní ma les aveugles marchent à l'aide d'un bâton

blè, blèc̀ $n$ Arachis hypogaea arachide [légumineuse annuelle, cultivée pour son fruit, la cacahouète, qui constitue sa graine et qui se développe sous terre] $\diamond$ blè yí zicn sauce 
d'arachide; blè pii pâte d'arachide ; blè gole déterrer l'arachide ; bònyí blè Desmodium adscendens arachide de brousse [non mangeable]

blinà loc gorge, dans la gorge $\square$ kaa bhelé dwà à blinàn l'arrête est coincée dans sa gorge $\square$ ǵ blinàn ò làzi ma gorge me fait mal $\square$ fétí ć ta bamu bhlàa bèbè blinàn kààn pendant la fête, les Dioulas ont égorgé beaucoup de moutons $\diamond$ blinàyicn tristesse ; è jàn wo à blinà il mâche les mots

bliy rn gorge (partie extérieure; de personnes, animaux) $\diamond$ bliy kúnle saisir la gorge, serrer la gorge

blinle, blinle $v \boldsymbol{t}(\mathrm{mob})$ lancer, jeter $\square$ Táatóo kpoti blinnà Blo ć ba Tato a lancé le caillou dans la rivière de Béré

bliyta loc à la décharge, aux ordures [il y en'a dans chaque village] $\square$ Màní klélé blin blinta Mani jette des ordures à la décharge

blò adv ideo boum (son de chute)

blòo $n$ Thryonomys sp. aulacode, agouti (Afr.) [gros rongeur dont la chair est très appréciée,

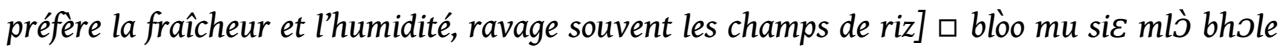
ma les agoutis ravagent les plantations de riz

bloole $v$ 1) vi 1. manquer (de - avoir besoin ma) $\square$ ó bloole laa ò pebhle ma nous ne

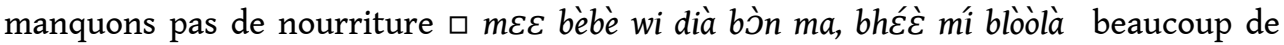
personnes ont tué du gibier à la chasse et moi, je n'ai rien eu 2. priver, dépouiller (de ma) $\square$ ý pebhle bloo é ma je te prive de nourriture

bloole vi 1. être débrousaillé (chemin) 2. casser, labourer, débroussailler (espace nouveau avec la machette, houe) $\diamond$ zi bloole frayer le chemin $\square$ ké ò zi kpáalé ò yre ma bloo còyyò lé quand on fraye un chemin, on débroussaille une place avec la machette

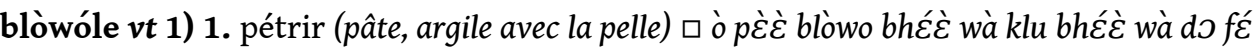
lé on pétrit l'argile, on la roule et on en construit des maisons 2 . brûler à cloques (les mains etc.) $\square$ gbo téle É ý gbelàa é blowòà le canari chaud m'a brûlé les mains à cloques 2) se brûler à cloques $\square y ́$ gbelàa é blowòa ma main s'est brûlée à cloques

blòn I $\boldsymbol{n}$ singe (poilu comme un mouton, avec une queue blanche)

blòn II $a d v$ pas du tout, pas l'ombre, pas une goutte

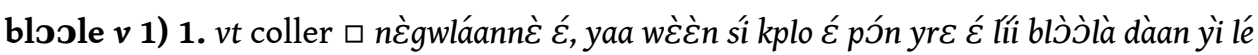
le vieil homme a collé le trou de sa gourde de vin avec du caoutchouc 2. attacher fortement $\square$ kplo $\varepsilon$ bloole ò sóo $\varepsilon$ ma la goude est attachée au vélo 2) vi 1. se coller $\square$ làanè $\varepsilon$ blòòla tásá $\varepsilon$ ma la feuille s'est collée à la cuvette $\diamond$ yrè líi bloo les yeux se collent; è bloว zà ma il exagère! 2. être attaché fortement 3. coller (à $m a) \square n \varepsilon ̀$ mu bloo ò neE mu ma les enfants se collent à leurs mères

blú, brúú $<$ Fr. boule $>\boldsymbol{n}$ pain [surtout un pain long] $\diamond$ blú yrí baguette ; blú yrí do un pain ; blú pii farine

bluy I $\boldsymbol{r n}$ nombril $\diamond$ bluy bhole avoir une hernie ombilicale $\square n \varepsilon \dot{\varepsilon}$ ć blun bhole ò ce petit a une hernie ombilicale

bluy II $\boldsymbol{n}$ jachère $\square$ ǵ gòo bole lec là án blun $\varepsilon$ ta cette année je vais semer du maïs sur mon terrain en jachère

bole $\boldsymbol{v}$ (const) 1) vi 1. rester $\square$ bo fé lé ma reste à la maison! 2. vivre $\square$ è bo wàla mle do là il reste au village un mois (tous les ans) $\diamond$ bole tré ta vivre 3. prospérer $\square$ bhé nój̀ bo àà ?

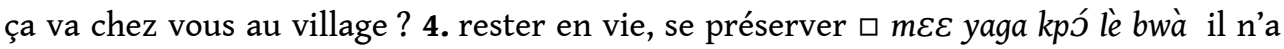


que trois personnes qui sont restées $\square$ zà ké do kpó láá bole sàyaàn mu ć kánle blaan ý de a fálà rien n'est resté dans la maison de mon père après le passage des assaillants 5. surveiller, veiller (sur - ba) $\square$ bo nc̀ ć ba surveille l'enfant 6. garder en mémoire (de qui - kpéé) $\square$ sò za mu ć bèbè bwà ý kpée j'ai gardé en mémoire beaucoup de pratiques d'autrefois 7. è bùa sa ké presque (lit. il restait un peu que) पè bwà sa ké ý báláá j'ai failli tomber 2) vi rester $\square \dot{y} n \bar{\varepsilon} \varepsilon$ ć bo Bíjà mon enfant va rester à Abidjan

bòníjazíi $\boldsymbol{n}$ danse (un des types de) [danse de réjouissance sans masques accompagnée de plusieurs instruments musicaux y compris la guitare; venue des Baoulés; les jeune filles nonmariées prennent part à cette danse activement]

bòò adj immature, non formé (fruit) $\diamond$ bòonć immature et petit $\square$ mángròo $\varepsilon$ wòo ébòonć lé ces deux mangues sont petites et non-formées

boo bènغ̀ $\boldsymbol{n}$ Sterculia tragacantha sterculia (arbre aux larges feuilles)

boo $r n$ 1. beau-parent (de l'homme; plus agé) 2. beau-père, belle-mère (d'homme) $\diamond$ boogwlesn beau-père (d'homme); boole belle-mère (d'homme); boomu beaux-parents (d'homme) 3. père du beau-fils ou de la belle-fille, mère du beau-fils ou de la belle-fille boolà $a d v$ après, plus tard $\square$ ké yá pià à ni boolazi ý gbú lè nu à kpén yoondele si tu le lui racontes, moi-même je traduirais après $\diamond$ na boolazimi femme cadette (en famille polygame); wóo boolazi à bientôt !

booya $<$ boo $+y a>n$ relations entre les beaux-parents (const) 1) vt 1. mettre (dans qqch) प 'y yá gòn ć bwà garazi yí j'ai mis ta voiture au garage 2. mettre (sur le poignet : bracelet, montre etc.) $\square$ kà ý mágbe, mí án mótró bo ý gbe ta attendez-moi, je vais mettre ma montre $\diamond$ drón bole emprunter $\square$ Màní gòli drón bwà à náágwlen kóó Mani a emprunté de l'argent à son grand frère ; win bole envoyer qqn $\square$ án wîn bwà gòli sí yre nò̀ je l'ai envoyé pour chercher de l'argent; ygblo bole opiner de la tête ; wee bole frétiller de la queue, remuer la queue ; zruy bole enrouler, s'enrouler $\square$ mle á zruy bo le serpent s'enroule ; wàanni bole jouer à l'awalé ; wànmi bole préparer des beignets de banane ; bàlo bole bavarder, causer 2) vi 1. monter (cheval, bicyclette - ta) $\square \dot{\eta}$ bo sòo ta je monte à vélo $\diamond$ bole gwláan $m u$ ba passer l'initiation $\square$ náágwlęn gbe bwà gwláan mu ba gban le fils de mon grand frère est déjà initié ; làkluù yí bole être scolarisé 2. se poser, se placer $\square$ gò $\varepsilon$ ह́ bo gàràzi yí d'habitude la voiture est dans le garage

bòmóó <Fr. bonbon> $\boldsymbol{n}$ bonbon

\section{bòmóóyàànnî $\boldsymbol{n}$ Mantis religiosa mante religieuse (insecte)}

bòn I $\boldsymbol{n}$ 1. savane, brousse $\diamond$ bònyí sauvage, de brousse ; gele bònma aller à la chasse $\square$ $m \varepsilon \varepsilon m u$ ć jà bòn $m a$ les hommes sont allés à la chasse ; bòn klcle faire la chasse 2. herbe

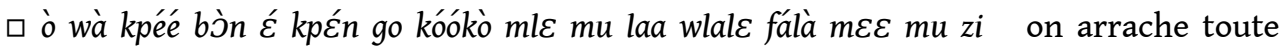
l'herbe dans le village afin que des serpents n'entrent pas dans les maisons 
bòn II $\boldsymbol{r n}$ 1. cerveau, cervelle (d'homme, d'animal) $\square$ ò bloo ýgblò wi bhéc̀ wà bòn bhle on casse la tête d'agouti et on mange sa cervelle 2 . moelle (d'arbre; des arbres à la moelle molle)

bòn pú $\boldsymbol{n}$ herbe (nom générique pour toutes sortes de grandes herbes vert claires)

bonle vi pourrir (de viande, fruits, légumes) $\square$ yromazi bhole plè̀ wi $\varepsilon$ nu bonle la viande va se gâter vers le soir $\square$ wi $\varepsilon$ bwàn bhee é líi l'animal a pourri dans le piège

bònmat $\varepsilon$ <b̀̀n+ma+tź> $\boldsymbol{n}$ chenille (esp. : couleur de feuilles, le contact est urticant)

bònml⿷ $n$ Dendroaspis viridis mamba vert (petit serpent vert : venimeux et très dangereux) 口 mle yòo le bònmle lé àmasròyí waa mado vè̀ bhćyaćyí à fle ć yré pegéé bòn yré ò do

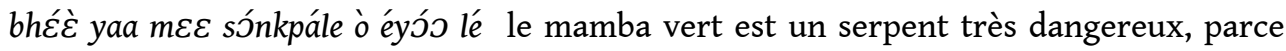
que il est de la même couleur que l'herbe, et c'est très difficile de le remarquer, tandis que sa morsure est très dangereuse pour les hommes $\square$ bònmle waa bhé bhle le mamba vert, on ne le mange pas

bòtò <Dioula> $\boldsymbol{n}$ sac $\square \diamond$ mlò bòtò sac de riz; bòtòné sachet, porte-monnaie (traditionnel); gbcýi bòtò sac qui est accroché au bras

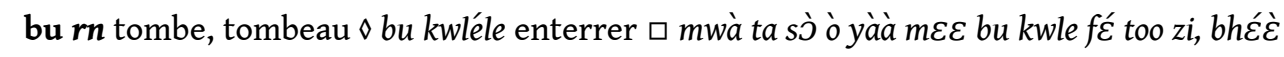
ò yàa nc̀ $m u$ bu kwle bòn ýi autrefois les Mwans enterraient les adultes derrière la maison, les enfants étaient enterrés dans la brousse

búàtî $<$ Fr. boite $>\boldsymbol{n}$ boîte (de conserve)

bùkénge $\boldsymbol{n}$ tortue d'eau $\square$ bùkénge mu ò ó ba wàla Blo ba, ké wôo kwân, wóo docie bhéċ wóo bhle il y a des tortues dans notre rivière de Béré, quand nous les attrapons, nous les faisons frire et les mangeons

bùybhàà, bùmàà $\boldsymbol{n} 1$. étoile la plus brillante (peut être une planète) 2. étoile du matin

bùsábùsá adv ideo grand

bútèlí $<$ Fr. bouteille $>\boldsymbol{n}$ bouteille

bùtìkí, bùtíkì $<$ Fr. boutique> $n$ boutique

bùu $n$ 1. tille (d'un bois) 2. fibre (d'un bois ou tissu) 3. planure, sciure (déchets de coupe)

búùné $n$ piège à poisson

bùzii $<F r$. bougie $>n$ bougie

bwee, bwe $n$ 1. poussière (sur la surface) $\diamond$ bwe gblè غ̀n poussière en air (lit. odeur de poussière) $\square$ voj bhla zi bwe gblèn ò ébèbè lé il y a beaucoup de poussière pendant l'harmattan 2. sable (sec qui couvre la terre)

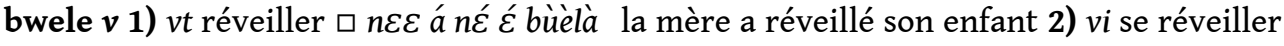
$\square$ wàla mo kpén bwe épléclé on se réveille tôt au village

\section{Bh - bh, comme bhaa, bhaa yá táá $\varepsilon$ nráa}

Bhaa é do <bhaa+é+do> intrj bonne chance!

bhaa $n$ fruit (d'arbre), noix $\square$ kpáko bhaa noix de coco $\diamond$ yri bhaa fruit

bhaa part marqueur d'optatif

bhaa $r v$ fructifier, porter des fruits (de plantes) $\square$ mángroó é bhaa le manguier porte fruits $\square$ án páá ć yà bhààlà do kpó ma calebasse a déjà porté des fruits 
bhaa yá táá $\varepsilon$ nráa <soi+ton+route+Art+bon> intrj bon voyage

bhaagbezu $n$ oiseau (esp.: petite poule de brousse à plumage roussâtre qui chante avant la pluie)

bhaama adv 1. pour rien, sans aucune raison $\nabla$ bhé láá sánle bhaama cela n'a commencé pour rien (le debut d'un conte traditionnel) 2. gratuitement $\square$ ǵ saflée nàa à ni bhaama je l'ai donné du savon gratuitement

\section{bhaapley $<$ bhaa+pley> $\boldsymbol{n}$ être humain}

bhaapú <bhaa+pú> $\boldsymbol{n}$ albinos [la naissance d'un albinos est considérée comme un malheur] $\square$ le é,è bhaapú do yàà la femme a mis au monde un albinos

bhàkúú adv ideo édenté

bhe adv 1. ici (par rapport à l'interlocuteur) 2. particule de discours $\diamond$ wáátí lá ba bhe dans le temps quand

bhee $\boldsymbol{n}$ funérailles, obsèques, commémoration d'un mort $\diamond$ bheekpàle funérailles

bhee $n$ piège, lacs (de liane, de corde) $\diamond$ glùyí bhee un piège posé à l'intérieur d'un trou (pour les animaux plus gros)

bhèèle $v(m o b)$ 1) vt 1. guérir (par un médicament, un remède) [dans la vision mwan, ce n'est pas le médecin, mais son médicament qui guérit] $\square$ nivakini ý bhééla la nivaquine m'a guéri

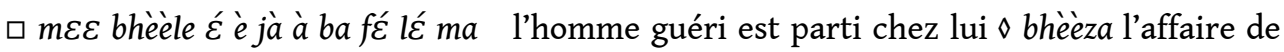
guérison 2. sauver $\diamond$ Christ. bhèeza salut; Bhèemi Sauveur 2) vi être guéri $\square$ gademi $\varepsilon$ ò bhéézí le malade va mieux

bhé kle ké conj ça veut dire, par conséquent, donc $\square$ ké wi é ven dwà bhé kle ké à śićle ò si la viande pue, ça veut dire qu'elle est gâtée

bhe $n$ 1. rn grain (du riz, maïs, sorgo), noyau (de mangue, d'avocat), tubercule (de manioc,

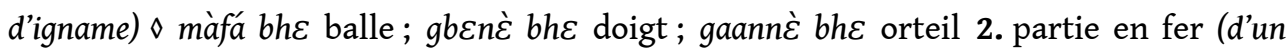
instrument tranchant) $\diamond$ wla bhe lame d'un couteau 3. un (indique la singularité des objets formant une paire ou des objets perçus comme une masse) $\diamond$ zrò bhe une abeille; yrغ̀ bhe do un œil 3. particule qui unit les numéraux des vingtaines avec les unités $\diamond$ mia ple bhe vu ta srwáple cinquante sept

bhe $n$ plaie $\square$ Nènć a bhe mláánà la plaie de Nené a enflé

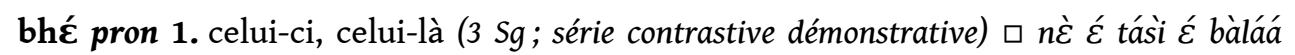

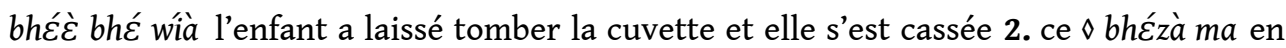
effet, à cause de ça ; bhéyí donc $\square$ kà ge bhéyí allez donc !

bhe $\boldsymbol{r n}$ ami, amie, camarade (d'habitude partie d'un mot complex) $\diamond$ bhegwlen ami ; ý bhenغ̀ $m u$ mes petites amies

bhéc̀ <bhé+là> pron celui, susmentionné pronom anaphorique contracté avec le marqueur du focus

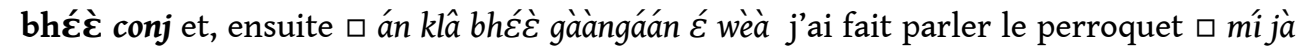
bhéċ è yi đià je suis allé et il s'est endormi

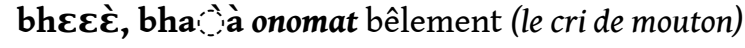

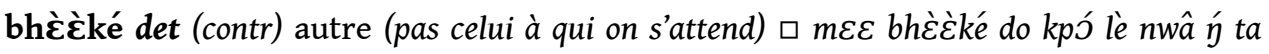
c'est une seule personne qui est venue chez moi (pas celle qui était attendue) $\square m \varepsilon \varepsilon$ bèc̀ ké $n \varepsilon \varepsilon$ lè kéle c'est la mère d'une autre personne $\diamond$ bhèc̀ké do l'autre (des deux), 
bhc̀c̀ké nále, bhغ̀c̀ké pwśle rembourser (rendre l'équivalent de ce qui avait été pris) $\square$ ý Táatóo à mlo ć bhéćké náa J’ai remboursé le riz à Tato

bhéغ̀male conj c'est pourquoi

bhéc̀yílè conj donc, c'est pourquoi $\square$ ǵ gòli bèbè yà bhéčyíle ý fé gbèntèn do dwà j'ai gagné beaucoup d'argent, c'est pourquoi j'ai construit une grande maison

bhéżzèćlè conj c'est pourquoi

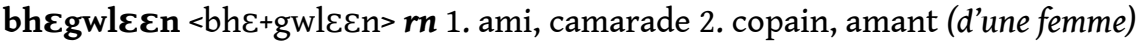

bhegwleqnya $<b h \varepsilon+g w l \varepsilon \varepsilon n+y a>n$ amitié

bhéláálè <bhé+láa+lè> conj ou, sinon

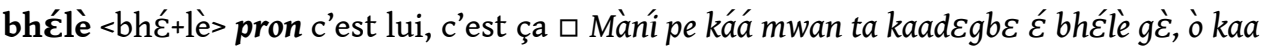
biná leyroy lé yi lâ Mani dit qu'en pays mwan, la manière de pêcher est comme ça: on repêche les poissons dans le fleuve avec les épuisettes

bhele $<\mathrm{bh} \varepsilon+\mathrm{le}>\boldsymbol{r n} 1$. amie 2. copine, amante

bhel lé $\boldsymbol{n}$ os (d'homme, d'animal) $\diamond$ wi bhelé os d'animal ; dreyí bhelé colonne vertébrale

bhémalè, bhéc̀malè <bhé+ma+lè> conj c'est pourquoi, à cause de ça $\square$ ga yàa à ma bhémalè yaa yàà so yewolè il était malade, c'est pourquoi il ne pouvait pas travailler

bhenغ̀ $n$ 1. $r n$ beau-frère (frère cadet du mari), ["mari à plaisanterie" qui est le mari potentiel en cas de décès du mari] 2. belle-sœur (femme du frère aîné), ["femme à plaisanterie »] \Syn. gbààṅ̀

bhéyaćyí <bhé+ya+ćtyí> conj car, en effet, puisque $\square$ waa mado vè̀ bhéyaćyí à flé pegéé bòn yré o do on ne le (serpent) repère pas tout de suite parce que la couleur de sa peau est la même que celle de l'herbe $\square$ Syn. àmasròyí

bhéyí <bhé+yí> adv ainsi, en effet, donc

bhezàmalè conj c'est pourquoi, à cause de ça

bhî pron toi (série emphatique)

bhîi conj comme (pareil) $\square$ à ýgblo ò bhîi kpákò bhaa sa tête est comme une noix de coco $\checkmark$ yò bhîi il semble, comme si $\square$ à ta ò édòj̀le zòn, yò bhîi ga à ma il est atonique aujourd'hui, on dirait qu'il est malade $\diamond$ yò bhîi épegée il faut que $\square$ yòo bhîi épegéé ge il faut que je parte

bhîi pron te (2 pers. sg., série emphatique non-sujet)

bhìle $v(m o b)$ 1) $v t$ allumer (lampe, électricité, gaz), mettre en marche (l'ordinateur ou qqch

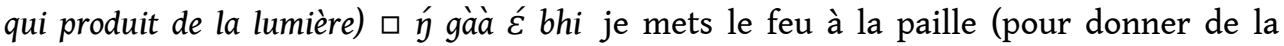
lumière) 2) vi s'allumer $\square$ gàà $\varepsilon$ bhi élrelé la paille donne beaucoup de lumière

bhlà manger (Perf. bhle)

bhla mensonge $\diamond$ bhla pele mentir $\square$ bhla pele ò éyóo lé mentir est mauvais; bhla kpále calomnier, diffamer (qui-ma) $\square$ ý ma bhlakpámi nwà l'homme qui parle mal de moi est venu; bhlapemi menteur $\square$ bhlapemi do à piâ ý ni káá bie mu si un menteur m'a dit que les éléphants savaient voler

bhla, mla $\boldsymbol{n}$ bêtise, manque de bon sens (propre aux enfants) [les enfants ne distinguent pas entre ce qu'on peut dire et ce qu'on ne peut pas dire; ils ne savent pas retenir leur gloutonnerie] $\checkmark$ bhla ò é yí tu es bête $\square$ gblanè é fálà jàn kpén pwe péc lii ; bhla laò à yì sa la jeune fille raconte tous les secrets de la maison, elle est trop bête 
bhla $\boldsymbol{n}$ temps, moment $\square$ pebhle bhla bhwà il est l'heure du déjeuner $\diamond$ bhla $\varepsilon$ dèla ba quand ? à quel moment ? वé nu yáa ye é léyan le bhla é dèla ba quand auras-tu achevé ton travail ?; bhla ké ba souvent ; bhla ع́ kpén ba toujours $\square$ Syn. wáátí

bhlà, bhlàà $\boldsymbol{n}$ mouton, brebis $\square$ flámu bhlà gblee les Peuls élèvent des moutons $\diamond$ bhlààda brebis ; bhlààgwleEn mouton ; bhlààné agneau ; bhlàgblèemi éleveur de moutons

bhlààtagbìnmi <bhlàà+ta+gbìn+mi> $\boldsymbol{n}$ Christ. pasteur $\square$ Syn. pàseli

bhlè $\boldsymbol{n}$ termite $\diamond$ bhlenáa reine des termites $\square$ Syn. bhlewín kpàa

bhlele $\boldsymbol{v t}(\mathrm{mob})$ manger $\diamond$ pebhlele manger (vi); gว̀li bhléle gaspiller (argent); yrèn bhléle souffrir; lè bhléle bouger les lèvres (d'habitude des animaux); lìi bhléle faire semblant de manger en remuant les lèvres

bhlemazi $\boldsymbol{r n}$ bordure (de chemin, champ, rivière)

bhlèggbè $\boldsymbol{n} 1$. dirigeant, chef [autrefois, un chef sacré qu'on transportait en hamac] 2. Christ. roi $\square$ Syn. fúá $\diamond$ bhlèngbèle reine; bhlèngbè f́ć palais ; bhlèngbè kpe trône 3. richard

bhlèngbèya $n$ Christ. royaume $\square$ án bhlègbèya, yaa yre laa ò gè ; án bhlèggbèa, yaa yre ò laaníima mon royame n'est pas ici, il est dans les cieux

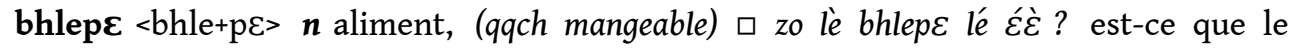
margouillat est mangeable?

bhlewi <bhle+wi> $\boldsymbol{n}$ viande, animal (mangeable) $\square$ bhlewi láá kwlen lé le caméléon n'est pas mangeable

bhlèwínkpàà $n$ reine des termites $\square$ waa bhle bhle dúy ò bhlèwínkpàa bhle, bhéċ bhé nra on ne mange pas termites, mais on mange la reine des termites, elle est bonne au goût 口 Syn. bhlènàa

bhle $\boldsymbol{n}$ 1. corde $\square$ Táatóo dri yrèla bhle lé Tato a attaché la vache avec une corde $\diamond$ zrugbhle coussinet (un morceau d'étoffe roulé en rond pour supporter une charge qu'on porte sur la tête); gbònbhle intestin; bhle bole attacher avec la corde (à - ma, sur - ta) 2. amulette au cou (cordon en cuir)

bhlé $\boldsymbol{n}$ Cephalophus sp. céphalophe (petite antilope)

bhleklo <bhle+ ?> $\boldsymbol{n}$ liane (grosse)

bhlén zro $n$ Tragelaphus angasi nyala (antilope de taille moyenne)

bhlíngán-bhlíngán adv ideo ça et là (de qqn se roulant par terre)

bhlo adj très jeune $\square$ gòo diin $\varepsilon$ bhlo nc̀ lè c'est un très jeune plant de maïs

bhlo $n$ assiette (en argile) $\square$ mwà mu bhlo lo gbàtó le mu kòj̀ les Mwans achètent les terrines des femmes sénoufo

bhlòó adv ideo miam (en mangeant à pleines poignées)

bhlò̀le $v \boldsymbol{t}(\mathrm{mob})$ presser, tordre $\diamond$ yi bhlóóle presser, extraire le jus, tordre (vêtement) ; lغ bhlóóle presser le bout de tissu mouillé

bhlo $n$ 1. $r n$ cou (d'homme, d'animal) $\diamond$ gbebhlo poignet; gaanbhlo cheville 2. guidon (de vélo, moto) $\diamond$ sóo bhlo attelage de chevaux

bhlù $n$ vipère [mangeable; la viande est parmi les plus recherchées; totem des Yahiri et de certains autres clans] $\square$ ý bhlù yren bhlà, bhlù yren nana j'ai mangé les œufs de la vipère, ses œufs sont bons

bhlùwlènné $\boldsymbol{n}$ chenille-vipère (esp.) 
bhlùzúỳ $\boldsymbol{n}$ Channa obscura lotte (poisson)

bhóbhó $n$ muet, sourd-muet (personne) $\square$ bhóbhó ple ò ó ba wàla il y a deux sourdsmuets dans notre village $\diamond$ bhóbhónć muet

bhodrùú $n$ Tilapia aurea tilapia bleu (poisson)

bhóflótò $\boldsymbol{n}$ pain rond, beignet (boules de farine frites à l'huile)

bhoo $\boldsymbol{n}$ cabri, chèvre (générique) $\diamond$ bhoo nàà chèvre (féminine) ; bhoo gwlecn cabri

bhòòlaple $n$ mercredi $\diamond$ bhöòlaple ma le mercredi

bhòkú-bhòkú adv ideo mou, moelleux (du pain, fruit très mûr, coussin, fauteuil etc.)

bhole $\boldsymbol{v}(\mathrm{mob}) \mathbf{1})$ vi $\mathbf{1}$. pousser (des plantes), germer 2. arriver u ò gòa blasànle É lé Bíjà ò bhwà gban Tùmòdi ils ont quitté Abidjan en courant, et ils sont déjà arrivés à Toumodi 3. arriver (de temps, saison) 4. aider (à - ba) $\square$ Màní nwâ án fé ć lâ kóókò ké è bho ý ba Mani est venu à ma maison pour m'aider $\diamond$ bhóńbami aide, Christ. diacre 2) vt 1. faire posser (des plantes) 2. tourner (une toupie), conduire (moyen de transport; ne se dit pas de monture ni de bête de trait) $\square$ ǵ gòn bhole daanzí j'apprends à conduire la voiture $\diamond$ trébhole être fatigué; tóbhole Christ. glorifier $\square$ kòó Zèzú tóbhó, Wàànbhaa gbe le glorifions le nom de Jésus, le fils de Dieu

bhu adj nombreux $\square$ ý $m \varepsilon \varepsilon$ bhu mu yà j'ai vu beaucoup de personnes $\square$ Sobbísi nránc̀ mu ò ébhulé les petits-enfants de Sobisi sont nombreux

bhulàle $v$ 1) vi se courber, se pencher $\square$ le mu bhùláá mlo kán yre noj̀ les femmes se sont courbées pour moissonner le riz 2. couver (d'oiseau) $\square$ maa bhulá á yren ta la poule couve ses œufs 2) $v t \mathbf{1}$. courber, pencher, faire se courber, faire se pencher $\mathbf{2}$. couvrir (un récipient) $\square$ ý tásá $\varepsilon$ bhúlàa gbo ć lii j'ai renversé la cuvette et couvert la marmite

bhwèle $\boldsymbol{r n}$ Péjor. coépouse (du même homme) $\diamond n \varepsilon \varepsilon$ bhwèle marâtre, autre femme du père (dans la famille polygame)

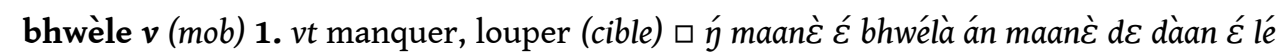
j'ai loupé l'oiseau avec mon lance-pierre 2. laisser échapper (du piège) $\square$ án gayyan É blò ć bhwélà mon piège a laissé échapper l'agouti

\section{C - c, comme ca, cácó, can}

6 ca $\boldsymbol{n}$ dette (de ce qu'on rembourse sans le rendre : argent, nourriture, etc.) $\diamond$ ca kpále prêter $\square$ Táatóo mlò ca kpàa ý ma Tato m’a prêté du riz; ca gbawole rembourser une dette $\square \dot{\eta}$ Táatóo à ca é gbawòa j'ai remboursé Tato ; ca lé à crédit

ca diin $\boldsymbol{n}$ Blighia sapida pommier d'aki, akée [arbre au tronc épais, dont les fruits sont très appréciés par les tisserins, qui y font leurs nids] càcà intrj attention! (avertissement)

cácó $\boldsymbol{n}$ cigarette (improvisée), [les feuilles sèches du tabac écrasées et enroulées dans des feuilles sèches de bananier]

9 can $\boldsymbol{n}$ 1. conte, récit (imaginaire) $\diamond$ can bole raconter des contes; glawò pegéé loonغ̀ a can le conte de l'hyène et du lièvre 2. Fig. événement irréel $\diamond$ yò bhîi can c'est comme un conte

càn $\boldsymbol{n}$ or (métal précieux et objets en or) $\diamond$ càn bh $\varepsilon$ grain d'or

cárá $n$ entonnoir [traditionnellement fait de feuilles de manguier ou de calebasses] 
cé adv 1. calmemant, sans bruit $\square$ ý nरे ple yaàle ye cर́ je vois deux enfants assis calmemant $\square$ bo cर́ ! soi calme ! 2. soigneusement, avec précaution $\square$ Zàle ò cèké tásá $\varepsilon$ ́ dulàzi cé Zalé place doucement la cuvette d'atiéké

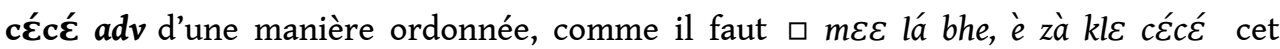
homme, il fait toutes choses de façon ordonnée

cغ̀ké <Baulé> $\boldsymbol{n}$ atiéké (Afr.) [sorte de couscous de manioc râpé et fermenté; traditionnellement inconnu des Mwans, ils l'ont emprunté aux peuples akan du sud]

cÈmò̀̀ $<$ Dioula $>\boldsymbol{n}$ vieux

Cغ̀saà $n$ mardi

ciè diin $\boldsymbol{n}$ Piliostigma thonningii pied-de-boeuf (arbre)

ciee $a d v$ sur le feu (en préparation)

cié, ciéé $n$ 1. invité, étranger (Iv.) $\diamond$ cié klele accueillir, honorer $\square$ kùnlii É yè ý cié klâ élrele le chef m'a bien accueilli 2. étranger

ciéya $<$ cíé+ya> $\boldsymbol{n}$ hospitalité $\diamond$ ciéya klele bien accueillir

cie rn 1. poil (d'animal, sur le corps humain) [les poils sur le corps sont vus comme un signe de

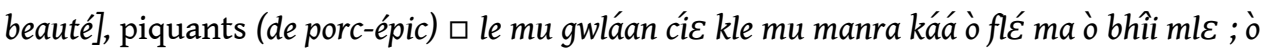
cí bhe do kpó láo ò ma les femmes se moquent des hommes sans poil, elles pensent que leur corps ressemble à celui du serpent, privé de poil $\diamond$ lèta cíc moustache; lèzàa cíi

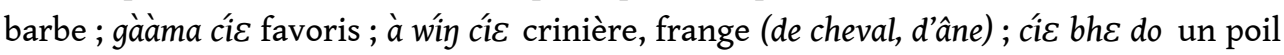
2. plume $\diamond$ maa cíc bhe do une plume d'oiseau 3. aile (d'insecte)

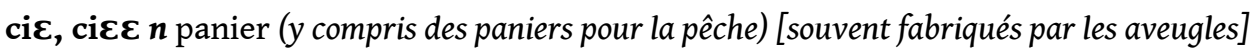

cién, ciéćn $\boldsymbol{r n}$ héritage (qu'on laisse après sa mort) [chez les Mwans l'héritage est distribué entre les enfants, par ordre d'âge (la part de l'aîné est plus importante), on ne fait pas de testaments. On hérite des champs de café, de cacao, des vetêments, et des fétiches, le plus souvent, l'argent n'est pas considéré comme objet d'héritage] $\square$ à cién kpén bòa à lù ni tout son héritage est revenu à sa fille

ciénle v 1) vt casser (en deux un objet long et dur, e.g. bras, bâton, pain dur) $\square$ le mu yj̀̀̀ cien les femmes cassent des fagots 2) vi se casser (d’objet long dur) $\square$ à gbe É ciénlà il s'est cassé le bras

cílo $n$ voir kílo

cóy-cóy onomatop tchac-tchac (bruit produit lorsqu'on coupe du bois)

còyyò, cuycuy, còygò $n$ machette (de fabrication industrielle)

có adv même

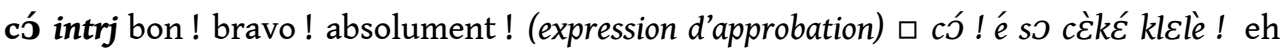
oui ! tu sais faire de l'atiéké !

cùycùy $n$ Arch. machette

\section{$D$ - d, comme da, dàan, dàancèneèné}

da $\boldsymbol{n}$ femelle (d'animal) $\square$ Syn. nàa

dà $n$ Hibiscus sabdariffa hibiscus (plante herbacée) [les feuilles fraîches sont la base de la sauce] $\diamond$ dà tén bissap (Afr.) 
dàan $\boldsymbol{n}$ 1. caoutchouc, plastique (matériaux élastiques et articles qui en sont faits) 2. lancepierre $\diamond$ maanc̀d $\varepsilon$ dàan lance-pierre pour chasser les oiseaux 3. seau en caoutchouc

dàancèyeèné $\boldsymbol{n}$ Ficus elastica caoutchoutier, plante à caoutchouc (de brousse) [plant à feuilles larges, épaisses et luisantes, utilisé pour produire le caoutchouc] $\diamond$ dàancèyè̀né yí jus du caoutchouc (utilisé comme colle)

daanlè $v$ 1. $v t$ enseigner qqn (faire qqch - Verb-le lé) $\square$ yà daanzí bhee bole lé il lui apprend à poser des pièges 2. apprendre qqch (ou faire qqch) $\square$ ý mùà wli daan j'apprends la langue mwan $\square$ ý bole daanzí sóofali ta j'apprends à monter à cheval $\diamond$ daanleya

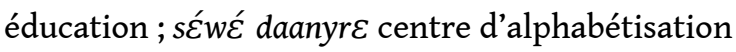

daanlere $n$ Acacia ataxacantha acacia (esp. piquant)

daanmi $<$ daan+mi> $\boldsymbol{n}$ maître, enseignant $\diamond$ tùàbù wli daanmi enseignant en langue française

dáású n Hystrix sp. porc-épic, hérisson (Afr.) [est chassé pour sa viande qui est très appréciée. Il est considéré un animal dangereux à cause de ses piquants qu'il lance dit-on quand on l'approche]

dáású wééwéné, dáású wéléwélé $n$ Atherurus africanus petit porc-épic, hérisson (IV.) [le poids 3-4 kg, mangeable, il est chassé pour sa viande qui est très appréciée]

dágbé <Dioula> $\boldsymbol{n} 1$. Hippotragus equinus coba, rufifron, antilope-cheval

dáídai adv ideo s'étalant, se vautrant (le façon d'être assis)

dale $v$ 1. vi venir de, revenir de $\square \grave{y}$ dazíi wáa wà $\varepsilon$ ta je reviens de notre village 2. être issu de, descendre $\square$ lenc̀ $\varepsilon$ da wàla ké kwé lé cette femme vient du village avec des

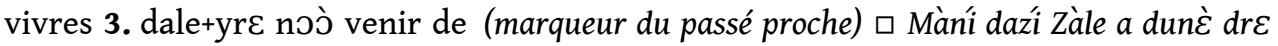
ple lò yre noj̀ Mani vient d'acheter deux nouvelles robes pour Zalé

dáma $<$ Dioula $>a d v$ plusieurs

dàn, dày $\boldsymbol{r n}$ limite, mesure $\diamond$ kánle dàn ta dépasser la mesure (Fig.) $\square$ è kààn dàn ta zòn,

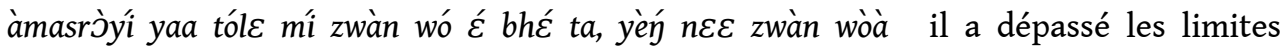
aujourd'hui car il ne s'est pas limité à m'injurer, il a insulté ma mère; ékánle dàntalé dépassant les limites, outre mesure $\square$ ǵn ne zwàn wó ć sía ý yí ékánle dàn ta lé l'injure de ma mère m'a blessé trop

dángán $<$ Dioula $>\boldsymbol{n}$ malédiction

danle vt (const) 1. peser, mesurer $\square$ án mlò bhe $\varepsilon$ dan! pèse mon riz! $\diamond$ danle kíló ta peser sur une balance 2. goûter $\square$ náá kutuku danle li do je n'ai jamais goûté de koutoukou $\diamond$ gwle danle se battre, lutter; danle à ǵgbhlo yì imaginer, planifier $\square$ yá pe ye lá bhí bhé dààné ýgbhlo yi ye bhe le travail que tu as planifié

dàwàà $a d v$ ideo étendu sur la terre (à la renverse)

dàwlí <Dioula> $\boldsymbol{r n}$ ruse, subterfuge $\square$ kéé dàwli ò zà láá é bhle si tu es malin, tu n'as pas de problème

dàwlíya $<$ dàwlí+ya> $\boldsymbol{n}$ ruse, malice $\square$ Loonc̀ a dàwliya laò sa la ruse du Lièvre est énorme dayre $<\mathrm{da}+\mathrm{yr} \varepsilon>\boldsymbol{n}$ nouvelles (de la maison, de village) $\square$ é dayre pe ó ni donne-nous de tes nouvelles

dè pron qui ? 
dèlà < dè+là> pron 1. qui (complément d'objet direct ou indirect) $\square$ è cùncùn drón bwà dèlà $b a$ ? à qui a-t-il emprunté la machette ? व é dèlà yezí laale ? qui vois-tu là-bas ? 2. quel ? à qui ? व dèlà nc̀ lè g̀̀ ? à qui est cet enfant ?

dèlè <dè+lè> pron qui ? (en fonction sujet) $\square$ dèle yá f́́ $\varepsilon$ dwà ? qui a construit ta maison ? $\diamond$ dèle kélè ? c'est qui ?

d $\varepsilon \boldsymbol{r n}$ 1. père $\diamond \dot{y} d \varepsilon$ zèzè mon propre père; $d \varepsilon m u$ parents ; nc̀ $d \varepsilon$ père d'enfant; $d \varepsilon$ ná femme du grand frère du père 2. Resp. père (adresse aux hommes de la génération des parents)

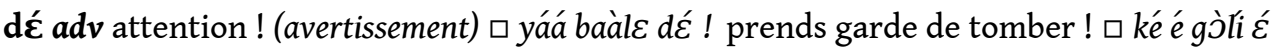
tóa gè è nu à fáánle dé attention, si tu laisses l'argent ici, il le volera

dè $\mathbf{\varepsilon} \boldsymbol{\varepsilon} \boldsymbol{\varepsilon} \boldsymbol{n}$ tracteur, bulldozer

$\mathrm{d} \boldsymbol{\varepsilon}$ gblan $<\mathrm{d} \varepsilon+$ gblan $>\boldsymbol{r n}$ oncle (grand frère du père)

dele $\boldsymbol{v t}$ (const) 1. tuer $\diamond$ kaa dele pêcher; wi dele chasser; yrì diin dele mettre le feu autour d'un gros arbre (pour le faire sécher en vue de faire un nouveau champ); yoon dele écrire; yi dele dormir; són dele se faire tatouer les gencives (Afr.) [pour des raisons d'esthétique; cette pratique des femmes mwans repandue auparavent était empruntée aux Dioulas] ; dewi animal (pour l'abattage) 2. battre, frapper $\diamond$ plin dele jouer le tambour ; gbe dele frapper dans ses mains, battre des mains, applaudir

deleya $<$ d $\varepsilon$ le+ya $>\boldsymbol{n}$ maladie, mal

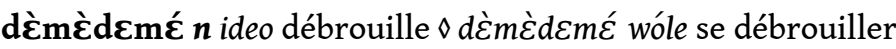

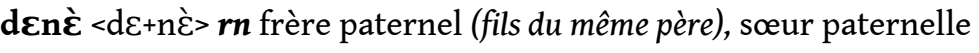

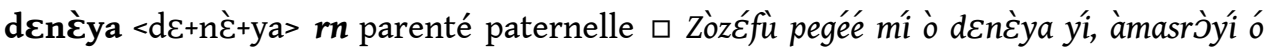
$n \varepsilon \varepsilon$ laa ò do Joseph et moi, nous sommes démi-frères, car nous ne sommes pas de même mère

di $\boldsymbol{n}$ inquiétude $\square \diamond$ di kan mú kpén ýi ils deviennent tous inquiets

di $\boldsymbol{n}$ lance, sagaie $\diamond$ di bhe sagaie, fer de lance

diin $n$ 1. plante $\square$ tùàbù yaannè diin la plante de pomme de terre $\diamond$ yrì diin arbre 2. régime (de banane, dattes etc.)

dímî $\boldsymbol{n}$ frustration $\diamond$ à dimí ò mEE ma personne frustré

dinî $\boldsymbol{r n}$ gencive $\diamond$ diní zon noircir les gencives [les femmes noircissent leurs gencives pour des raisons d'esthétique]

dìní $\boldsymbol{r n}$ jupe du masque (des hommes) [jupon de fibres faites de palmier raphia; ce jupon peut être long ou court selon le type de masque]

dìn $\boldsymbol{n}$ espace à côté, espace près de qqch $\square$ fर́ $\varepsilon$ diy $\varepsilon$ tríy ò à côté de la maison c'est sale

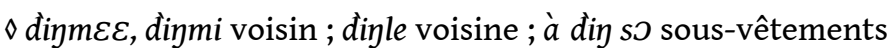

dìy pstp 1. à côté de $\square v \dot{\varepsilon}$ diin diń à côté du fromager

diy onomat boum! (bruit produit par qch. qui tombe)

diyme $\boldsymbol{n}$ voisin

diskó < $<\mathrm{Fr}$. disco $\boldsymbol{n}$ disco (type de la danse moderne)

Dìwì $\boldsymbol{n}$ fétiche (on lui offre un chien comme sacrifice) [en forme de cloche en fer avec une manche; un des féticheurs sonne le fétiche et les autres tuent le chien ; le sang du chien est versé 
sur le fétiche; la viande du chien est préparée et consommée par toute l'assistance] $\square$ Diwì gba mu waa yo gba gbàan lé ceux qui adorent le Diwi lui offrent un chien comme sacrifice

do num un $\diamond$ do $\varepsilon$, do kpó ć unique $\square$ mí án dunè pú lréle do ć sía j’ai mis mon unique joli habit blanc

do det 1. quelque, certain $\diamond m \varepsilon \varepsilon$ do quelqu'un, une personne; do kpó un seul, aucune

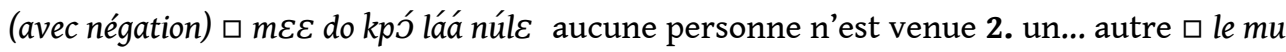
yalá gbe do, gwláan mu yalá gbe do les femmes s'assoient d'un côté, et les hommes s'assoient de l'autre côté 3. le même $\square$ yée wèc̀n do s̀ yéle kan bhé zi ć zi c'est le même bangui qui est servi à ces occasions

do do < do+do> det chaque $\diamond$ kpén do do chaque, chacun $\square$ nè bèbè ò ý kóó bhéċ o kpén do do ò làkluù yì j'ai beaucoup d'enfants, et chaqun va à l'école

dó $\boldsymbol{n}$ danse (danse des hommes; intensive et difficile à exécuter)

dòò $n$ courge

dóó $\boldsymbol{n}$ douche (à ciel ouvert), toilettes (là où on urine) [située derrière la maison au village; clôturée par un mur de feuilles de palmier, de bois ou de pellicule plastique]

doo $n$ perdrix, poulet de brousse

dò̀le < dòò+le> $\boldsymbol{r n}$ sœur aînée, grande sœur (Afr.)

dosò $<$ Dioula $>\boldsymbol{n} 1$. chasseur $\square$ Syn. widemi

dòwá ỳri $\boldsymbol{n}$ Holarrhena floribunda holarrhène du Sénégal

do $r n$ 1. membrane, bloc, barrage (de passage dans des trous d'animaux) $\square$ do moว ć do tii place-toi devant la sortie de secours de la souris 2 . hymen $\diamond$ do pónle déflorer

do $r n$ part

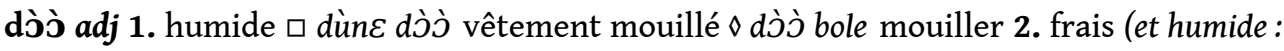
plantes, herbes, légumes, vent, temps) $\square$ plò dò kánzí un vent frais souffle $\square$ è dà dj̀̀̀dj̀ É yâ gba É ýi il a vu l'oseille de Guinée fraîche dans le champ 3. Fig. sain, en bonne santé $\square$ Zalè ò édj̀ lé Zalé est en bonne santé 4. clément, calme (personne) $\square$ Màni ta ò édj̀ò lé ż̀n Mani est calme aujourd'hui $\diamond$ dòj̀ bole être tranquille, Christ. être en paix $\square$ bhaa é dòj̀ bo ! sois en paix ! 5. agréable, plaisant

doo conj 1. que (introduit le discours direct) $\square$ è pe doo : il dit que : $\diamond$ doo ká doo que 2.je dis, il dit (marqueur reportatif)

doo $n$ Isoptera termite ailé

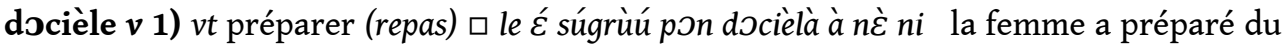
foutou de manioc pour son enfant 2) vi être préparé (nourriture) $\square p \varepsilon$ É docielà gban la nourriture est préparée

doole v 1) vi 1. être droit, devenir droit $\square$ zi $\varepsilon$ doole ò c'est un chemin droit $\square$ zi $\varepsilon$ doozí le chemin devient droit 2. être correct, être juste $\square$ à gbe plemi $\varepsilon$ dò̀̀la son deuxième fils s'est redressé 2) $v t$ redresser

dòòmá $<$ Dioula $>d t m$ la plupart

doon $\boldsymbol{r}$ trachée, œsophage [les Mwans ne distinguaient pas les voies respiratoires de l'œesophage] $\square$ yi ć káàn à doon yí il a bu de travers $\square$ yi ć bwâa à doon yí il s'est étranglé avec de l'eau

doondoon $n$ Sphecidae guêpe-maçonne $\square$ doondoon a fर́ kpa la guêpe-maçonne fait son nid 
doonle $v \boldsymbol{t}$ mettre en tas $\square$ ò kwléEn doon gba bhlemazi le coton est mis en tas en bordure du champ

$\operatorname{dogb\varepsilon } \boldsymbol{n}$ forme (aspect extérieur) $\diamond$ dogbeya vue externe; dogbe dre wole modeler,

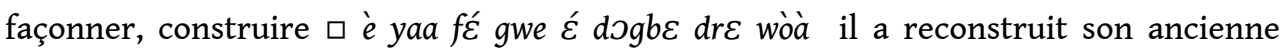
maison

dòké adv dòké... oo sans aucun doute, probablement, peut-être $\square$ bi pwà gban, dòké kòó $d \varepsilon$ ò zan zi líi oo la nuit est déjà tombée, notre père est probablement en train de venir $\square$ dòké è nu à lèpále oo sans doute, il va le rencontrer

dole v 1) vt 1. mettre (sur une surface qui n'est pas plate), accrocher (sur le mur) $\square$ p $\varepsilon$ ć do f́́ gwle ma! mets ça au mur ! $\diamond$ dole à wîin charger sur la tête de qqn $\square$ nu à do ý wîin viens me le charger sur la tête; dole à là être d'accord, approuver, accepter $\square$ è dùa dùtii a kítí É lâ il a approuvé le jugement du chef; do à zita soutenir qqn; dole kpéé se rappeler (après avoir oublié) $\square$ Màní a le síle zà dùa ý kpéé je me suis souvenu du mariage

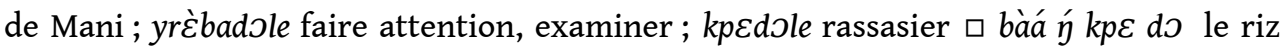
me rassasie 2. construire $\square \grave{\eta} d \varepsilon$ le fé léc dwà c'est mon père qui a construit cette maison 3. mettre (lunette, ceinture, foulard) $\square$ à do é yré ta mets-la (lunette) sur tes yeux $\square$ yá jrà É do é dì mets ta ceinture ! 2) vi 1. s'arrêter $\square m \varepsilon \varepsilon$ É dole ò vè diin là l'homme

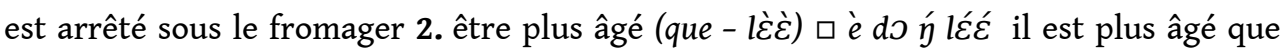
moi 3. être bâti (de ville, village) $\square$ Bijà dozí weỳi din Abidjan est construite au bord de la mer

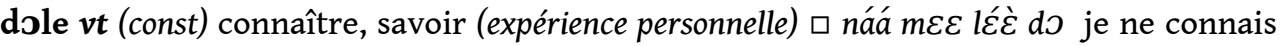
pas cette personne $\diamond$ zàdole intelligence $\square$ án zà dole yìya ý bhégwlen ni je suis plus intelligent que mon ami ; zàdowéć regret

don $\boldsymbol{n}$ furoncle, abcès

dòn $\boldsymbol{r n}$ pâteuse élastique, pâteuse gluante (qqch qui file: salive, fromage fondu, sauce gombo) $\diamond$ dòn kánle $v t$ abréger, couper (pâteuse gluante) $\square$ zièn $\varepsilon$ dònkán! coupe la sauce! 口 yá jàn é dònkán sois bref !

drà $n$ mare (dans la brousse; petite avec de l'eau stagnante)

dré $\boldsymbol{n}$ Ricinus communis palmcrist [les feuilles servent comme base de sauce]

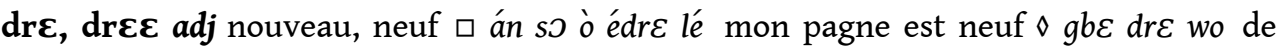

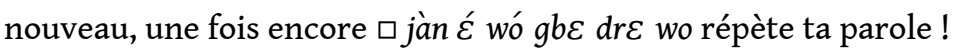

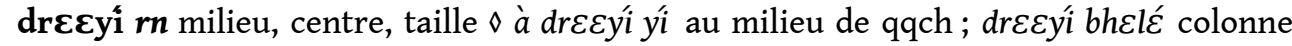
vertébrale; drecyí bhlele danse (espèce de)

drદyí gbèè $r n$ nageoire dorsale

dri $\boldsymbol{n}$ bœuf, vache (générique) [les Mwans n'élèvent pas de vaches] $\diamond$ gàa dri pile de paille

dridemi $\boldsymbol{n}$ boucher

drikJon $n$ Auchenoglanis occidentalis poisson-chat

driyoonle klo $n$ Anthia Carabidae carabe

drò $<$ Dioula $>\boldsymbol{n}$ vin rouge

dro $n$ buffle

droón $a d v 1$ 1. tout à fait, seulement, même $\square$ à kle droón fais seulement ça (ne te distrais pas) 2. du tout (avec négation) $\square$ bò̀ fiéntrònné lè, waa vli ye à ỳi droón c'est une petite forêt, on n'y trouve même pas un rat 
dròdróò $<$ Fr. docteur> $\boldsymbol{n}$ médecine, traitement (du type européen) $\diamond$ dròdrój bhe pilule, comprimé ; dròdró̀̀mi médecin ; dròdrój̀ fć hôpital

drón $\boldsymbol{n}$ dette (argent, riz etc., qu'on rembourse par la même somme ou la même quantité) $\diamond$ drónbole emprunter; drónkpále prêter

drùanع rn (coord) neveu (fils de sœur : pour l'homme qui est l'oncle maternel ti)

drùanc̀le $\boldsymbol{r n}$ nièce (fille de la sœur ; par rapport à un homme; la sceur du neveu drùanc̀)

drúnyán, drúyan <Dioula> $\boldsymbol{n}$ 1. monde (univers) 2. avenir (de qqn) प Yóo é kpéé lé-danzí yaa drúyán maà ? penses-tu à son avenir ?

droàle, drwàle $\boldsymbol{v}(\mathrm{mob})$ 1) vi être froid, se refroidir $\square$ yi drwâle na ý ni donne-moi de

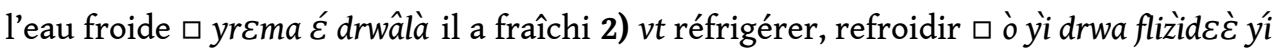
tùàbù tré ta en ville, on fait refroidir l'eau dans des frigidaires

du $n$ 1. Syncerus caffer buffle 2. Connochaetes taurinus gnou

dúàmásá <Dioula> $\boldsymbol{n}$ Gyps rueppellii gyps de Rüppell

dulàle $v$ (const) 1) vi 1. s'arrêter $\diamond$ dulà sa attends un peu; dulàle o être debout; dulàle yre Math. multiplier $\square$ ple dula yre ple, bhé kle yizic deux fois deux font quatre 2) vt 1. arrêter $\square$ ó ye é dùláá yrèkpáa nous avons arrêté le travail à midi 2. vt mettre, poser (sur la surface) $\square$ nEE tásá dùláá tàbàli ta ma mère a mis la cuvette sur la table

dúléý <Dioula> $\boldsymbol{n}$ ligne $\diamond$ dúlén bhe hameçon; dúléy yrí canne à pêche, ligne de pêche ; dúlén bole pêcher à la ligne u ò yàa gezí yi ć ta kón é yí, ké ò ge dúlén ć bole lé ils allaient sur l'eau dans la pirogue en pêchant à la ligne

dúmá $<$ Dioula $>\boldsymbol{n}$ bénédiction

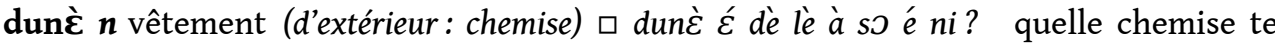
plaît? $\diamond$ dunc̀ gboon boubou (longue tunique ample, traditionnelle pour dioula, gouro et d'autres ethnies), robe ; dunc̀ kpeteekpe camisole (Afr.)

dunudu adv ideo Péjor. gros (personne, animal, insecte) $\square$ Zàle ò kpeteekpe bhéc̀ yò dunudu Zalé est petite et grosse $\square$ Syn. blaale

dùy adj profond (eau, réservoir) $\diamond$ yi dùn l'eau profonde $\square$ toflà ć dùy klâ le fleuve est devenu profond

dúy conj mais $\square$ Fónsì yaa gòy srá ć bhé sía gban, dúy yaa nule li Alphonse a déjà pris son transport, mais il n'est pas encore arrivé

dùynùybá, dùnùbá <Dioula> $\boldsymbol{n}$ tambour (esp. : cylindrique, à deux membranes)

dùyté $n$ Diceros bicornis rhinocéros

dùté $<$ Fr. du thé> $\boldsymbol{n}$ thé $\diamond$ dùté yí thé (boisson)

dùtìi <Dioula> $\boldsymbol{n}$ chef de village [désigné par le propriétaire de terre tré zan]

duu adv ideo de grande ouverture

duunle $v$ 1) $v t$ accrocher, pendre, suspendre $\square$ mí án bòtò $\varepsilon$ dùunlà yrì $\varepsilon$ ma j'ai accroché mon sac à l'arbre 2) vi être accroché, être suspendu $\square$ wò̀ dun yrì ma le singe s'accroche à l'arbre $\square$ dunc̀ $\varepsilon$ dunle ò la chemise est accrochée

dwà $n$ tourterelle

dwàléy $<$ Dioula $>\boldsymbol{n} 1$. miroir $\diamond$ dùàléy yí dans un miroir 2. vitre, verre (substance)

dwani, dwanii $\boldsymbol{n}$ araignée (venimeuse) $\square$ duani a páálà bhéc̀ nìini kláa à ta il a été piqué par une araignée et il a la fièvre 


\section{E - e, comme é, é taá, éblaan}

é pron 1. tu (2 pers., sing.; série sujet de base) 2. te, ton (série non-sujet)

é pron lui-même (3 pers., sing. ; pronom réfléchi)

è pron il (3 pers. sg. ; série sujet de base)

é taá <Dioula> $\boldsymbol{n}$ il faudra que tu... (formule de politesse) $\square$ náwé, é taá ge fétí bhléle ý ba grand frère, il faudra que tu viennes à notre fête

éblaan <é+blaan> $a d v$ encore, aussi, de nouveau (répétition d'action) $\square$ Syn. gbe dre wo

éke <é+ke> $\boldsymbol{n}$ l'un l'autre $\square$ wòo éke ye ils se voient $\square$ gaanle so $n \varepsilon \grave{m u} n i$ éke lèc̀ les enfants aiment se cacher les uns des autres $\square$ ò zàblé kle éke ni ils se font des cadeaux $\diamond$ éke lèyéle se rencontrer $\square$ Syn. ké

ékelé pron ensemble (deux participants d'action)

ékezi pron ensemble, tout le monde ensemble (plusieurs participants d'action) $\square$ ò sró kpa éke zi leglizi fálà on chante ensemble dans l'église

épegéé prep comme, comme si c'était $\square m \varepsilon \varepsilon$ do $\varepsilon$ bhé láàbo épegéé nc̀ l'autre le soulevait comme on soulève un bébé

éyáà $a d v$ hier

éyáàgè $a d v$ la dernière fois

éyatoma $a d v$ avant-hier

ézìy $a d v$ encore, aussi, de nouveau (répétition d'action) ч Syn. gbe dre wo

$\varepsilon-\varepsilon$, comme $\dot{\varepsilon}, \dot{\varepsilon} \varepsilon$

É det le, la (article défini)

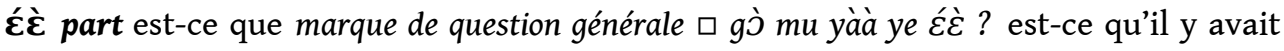
des chimpanzés?

\section{$F$ - f, comme fáá, fààn, fáánle}

fáá $\boldsymbol{n}$ chapeau (de l'homme) [le chapeau mwan traditionnel était le bonnet cousu de tissu traditionnel de coton] $\diamond$ fáa wlále mettre un chapeau

fàan $n$ vol (cambriolage) $\square$ ý fààn tó kpáa à ma je l'ai accusé de vol $\diamond$ fàan wóle voler, commettre un vol ; fàànwómi voleur ; fàanma $p \varepsilon$ chose volée

fáánle vt 1. voler (chiper) 2. détrousser qqn $\square$ náá yàa yáán go bhîi wò ý diymi ć fáánnà bi je ne savais pas que mon voisin avait été cambriolé

fàànnîí, fàànnî́ $\boldsymbol{n}$ voleur $\square$ Syn. fà̀nwómi

fáázan $<$ fáá $+z a n>\boldsymbol{n}$ policier, militaire

fàfà $\boldsymbol{n}$ matelas, natte (traditionnelle, grosse, faite de feuilles de palme) [utilisée aux champs pour le repos] $\square$ PJ yJ fáfa là tan à yilàpe lé Po tresse une natte de feuilles de palme pour dormir

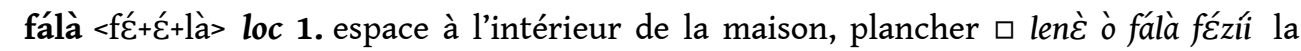
femme est en train de laver le plancher de la maison 2. dans la maison, à la maison 
fàlí $\boldsymbol{r n}$ sabot

fán $\boldsymbol{n}$ calao (oiseau) [possédant un bec très long ainsi qu'un plumage vivement coloré].

fáne $n$ Eutropius niloticus eutropius (esp. de poisson)

fansî $<$ Eng. fancy> $\boldsymbol{n}$ tissu de coton, fancy (Afr.)

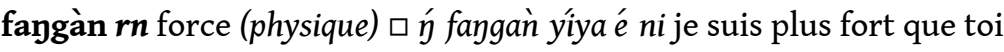

farínì $<$ Fr. farine $>\boldsymbol{n}$ farine (surtout la farine de blé importée)

fe $\boldsymbol{n}$ butte $\diamond$ fe yì kpále biner des buttes $\square$ yò fe yì kpázíi il est en train de biner des buttes

Fe pá mle $n$ mai (le mois de faire des buttes) $\diamond$ Fe pá mle lé au mois de mai

féé adv ideo un peu (en perçant une ouverture, un trou) $\square$ bhé pónle ò gèe feénć ceci est percé comme cela tout petit

fee $\boldsymbol{n}$ écale (de noix comestible), la première couche de la branche de palmier

fèlà $\boldsymbol{r n}$ sabot (d'animal) $\square$ Syn. fali $\diamond$ gaan felà sabot de derrière; $g b \varepsilon$ felà sabot de devant

féy-féy adv ideo entièrement, complètement (de qqch fini, dépensé, consommé)

fétغ̀n $\boldsymbol{n}$ poinçon (instrument pour percer le palmier pour prendre sa sève)

fé $\boldsymbol{n}$ maison, immeuble (la forme contractée avec l'article : fá) $\diamond$ félíi porte de la maison (baie) ; fÉkpéé chambre ; fÉwiiin toit ; pEgonfé boutique ; làkluu fékpée do classe (à l'école)

fédomi $<f \varepsilon^{\prime}+d O+m i>n$ maçon

féćntròn adj petit

fégwle <f́́t+gwle> $n$ mur $\square$ mwà $m u$ waa fégwle do pèc̀ lé les Mwan construisent les murs avec de l'argile $\square$ piibh $\varepsilon$ f́́gwle mur en fer

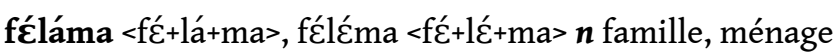

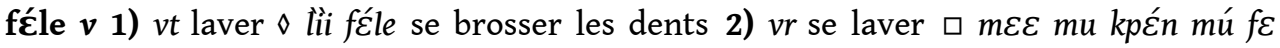
zrwanzi tout le monde se lave le matin $\diamond$ f́́le yi ba se baigner (dans le fleuve)

félíîkón <fé+lií̀+kóy> $\boldsymbol{n}$ porte (en bois)

feyget $\varepsilon$ adv ideo faible (homme, animal), léger (objet)

féti $<$ Fr. fête $>\boldsymbol{n}$ fête (pas traditionnelle)

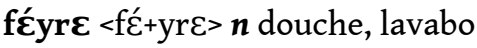

fién adj petit

fiénnè <fién+nc̀> adj petit

fii diin $\boldsymbol{n}$ arbre (esp. : donne des fruits mangés par les oiseaux)

finćtì <Fr. fenêtre>. $\boldsymbol{n}$ fenêtre [il n'y a pas de fenêtres dans les maisons traditionnelles des Mwans]

fitràn $n$ lampe à huile (traditionnelle) $\diamond$ fitràn kpá gbonc̀ jatte de la lampe à huile

flá $\boldsymbol{n}$ peul (ethnie, langue) $\diamond$ flámi Peul (représentant de cette ethnie)

flálron $n$ varan (grand lézard carnivore)

flán rn 1. personnes de même âge (personnes qui avaient fait l'initiation ensemble) $\diamond$ flánmado personne de même âge 2. génération

flànàflòlò $n$ 1. Anomalurus sp. anomalure, écureuil volant (rongeur arboricole de la famille des anomaluridés, à queue écailleuse, vivant en Afrique tropicale et pouvant effectuer des 
vols planés) 2. hermaphrodite 3. Fig. homme irrésolu (qui ne peut pas faire le choix; âne de Buridan)

flanغ̀ n Abelmoschus esculentus gombo $\square$ flanè zien sJ mo kpén ni tout le monde aime la sauce gombo

fláóflao $a d v$ = tén fláóflao orange, jaune (feux de circulation)

flééle v 1) vi être propre, devenir propre $\square$ mí tríy dwà, bhí fléelà moi, je suis sale, toi, tu es propre 2) $v t$ rendre propre $\square$ ý yáa dunc̀ ć flééla j'ai rendu ton habit propre

flè̀ adj (contr) vide $\diamond$ yi flè vide à l'intérieur $\square$ bútèli $\varepsilon$ yí fléy ò la bouteille est vide $\square$ klòy glù É yí fléy lè yàà le puits était vide; ma fley nu ; ta flè̀ dénudé (à torse nu); dì fley sans caleçon, à derrière nu; kóó fley pauvre (pas riche) ם zà la kóó fléy mu ta la vie est dure pour les pauvres ; kóf́flenmi pauvre, indigent

flé, fléć $r n$ peau $\square$ bhlàa flé $\varepsilon$ go enlève la peau du mouton $\diamond$ yrèflé paupière ; lèflé bout des lèvres, lèvres ; à lèflé ò il est bavard

flíy $n$ Digitaria exilis fonio [céréale cultivée dans le Sahel, qui donne un grain très fin et dont on prépare le couscous et des bouillies]

flísáflisaa adv ideo crépuscule $\diamond$ zrwanzi flisáflisaa crépuscule de matin

flizìd $\varepsilon \grave{\varepsilon}$, flizìd $\varepsilon \grave{\varepsilon}<$ Fr. réfrigérateur $\boldsymbol{n}$ réfrigérateur

flóy intrj nez percé ! (injure : signe d'esclave) $\square$ Vulg.é flóy tu as le nez percé !

flùgbà $n$ personne paralysée, paralytique $\square$ è baàle yrì ć yí ké è klelè flùgbà lé il est tombé de l'arbre et il est devenu paralysé

fóǹ $\boldsymbol{r n}$ faute $\square$ é fó̀ lè gè c'est ta faute $\diamond$ fón ta de la faute de, à cause de, de la fait de (qqn) $\square$ à fón ta té bùa gba é yí c'est de sa faute si le feu s'est allumé dans le champ

fóó adv ideo largement (en perçant une ouverture, un trou) $\square$ án pwàn fóo j’y ai percé un grand trou

fònó $<$ Fr. fourreau $>\boldsymbol{n}$ fourreau boîte $\diamond$ táklá foró boîte d'allumettes

fótó $\boldsymbol{n}$ banane (sorte: qui se colle) [banane plantain de forme spécifique; tabou pour certain groups totémiques]

fòtoò $<\mathrm{Fr}$. photo $>\boldsymbol{n} 1$. $r n$ photo, photographie 2. portrait, image $\square$ yòo griinc̀ géé, bhéc̀ $m \varepsilon \varepsilon$ fotoo ò à ta ce sont des petits ronds avec un portrait d'un homme à la surface 3. écran (de télévision)

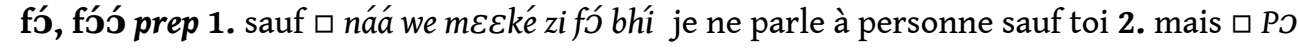
a fé ć yaa ò gàa fé lé fó tóli fé la maison de Po n'est pas une paillotte, mais une maison

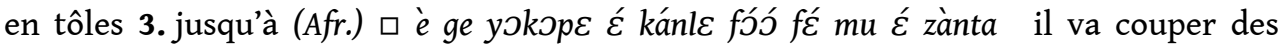
branches de palme jusqu'à derrière les maisons

\section{fòòfóó $\boldsymbol{n}$ poumon}

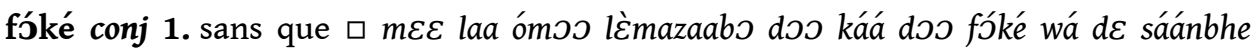
personne ne discute avec nous sans que nous ne le tuions sur place 2. si... ne, sauf si (Afr.) $\square$ ý nu à zonle fóké è gwà wà ć ta je vais le frapper s'il ne quitte pas le village

fòkú-fòkú adv ideo doux (pas dur)

fónfóné n Causus maculatus vipère de nuit [fréquente autour des villages]

fòy $a d v$ doucement, lentement $\diamond$ fònnć minutieusement, doucement ; à kle fòy! sois prudent, fais attention! $\square \grave{a}$ kle fòy yáá baàle fais attention, ne tombe pas! 

lezipe aussi l'autre

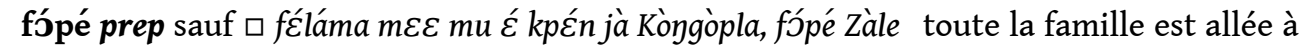
Kongasso, sauf Zalé

frò <Dioula> rn Vulg. bourses, scrotum (peau qui enveloppe les testicules)

frós $\varepsilon$ <Fr. forcé> $\boldsymbol{n}$ travail forcé

frú, frúú rn dot (biens payé par la famille de l'homme pour l'épouse) $\diamond$ frú wole payer la dote; frú kúnle accepter la dot $\square$ yà lù frúú ć kwân il a accepté la dot de sa fille $\square$ Syn.

frúú adv ideo vite

fùá $n$ roi $\square$ Syn. bhlèggbè

fúywóle $n$ plante (esp. ; les feuilles sont utilisées dans les sauces) $\square$ fúywóle kpée go élrèlé " funwolé » est bon pour le ventre

fúú n 1. rn force, capacité, possibilité $\diamond$ fúú òy ta j’ai de la force ;ýfúú so é ta je peux te

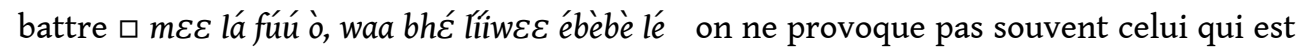
fort $\square$ a fúú swâ sánle ta yo ć yí il est arrivé à grimper jusqu'en haut du palmier $\square$ à fúu láá ò sólé bhoo é kúnle ta il n'est pas capable d'attraper le cabri (lit. : il n'arrive pas) ; à

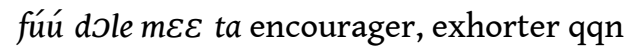

fúú $n$ sueur $\square$ fúú bho yewomi mlè ma la sueur apparaît sur le front du travailleur $\diamond$ fúúo ý ma j'ai chaud $\square$ fúú ven ò é ta tu sens la sueur

fway $\boldsymbol{n}$ aluminium, étain (métaux légers différents ou objets qu'on en fait)

\section{G - g, comme ga, gàà, gààbà}

ga $\boldsymbol{n}$ maladie $\diamond$ ga ò à ma il est tombé malade $\square$ ké ga ò $m \varepsilon \varepsilon$ ma yaa pe bhle si quelqu'un est malade, il ne mange pas $\diamond$ ga kpàa à ma il est devenu malade $\square$ dwani Dávidi páalà bhémale ga kpàa à ma une araignée venimeuse a piqué Davide et il est tombé malade

gàà $\boldsymbol{n}$ 1. herbe de brousse (esp.) [herbe dont les jeunes pousses sont très piquantes] 2. paille,

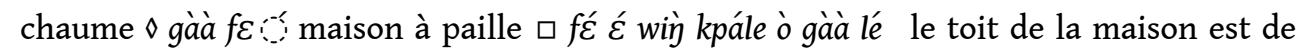
chaume 3. toit (de chaume)

gàà $r n$ joue $\diamond$ ka gàà branchies; gààma cíc favoris ; gààdole gifler $\square$ Christ. ké wò é gbe kò ta gàa dwà, é gbcyicta gàa do ò ni si quelqu'un te gifle sur la joue droite, tends-lui

gààbà $r n$ joue ronde $\square n c \grave{\varepsilon}$ gààbà láo sa cet enfant est très joufflu

gààbhelé rn mâchoire

gààkpámi <gàà+kpá+mi> $\boldsymbol{n}$ couvreur (spécialiste des toitures en chaume)

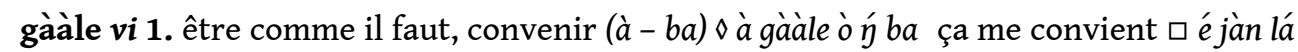
wózí bhe, bhé gààle lào é ba ce que tu dis ne te va pas $\diamond$ ò gààle ò éke yí ils se conviennent bien 2. aller (de vêtement; à qui - ba) $\square$ yá dunc̀ ć gààlà é ba ton vêtement te va 3. être conforme $(\grave{a}-b a)$

gaankpoygbo $<$ gaan+ ? $>\boldsymbol{n}$ talon

gaan $\boldsymbol{r n}$ pied (homme, objet), jambe (homme), patte de derrière $\diamond$ gaann⿳亠े bhe orteil, doigt de pied; gaanbhlo cheville; gaanwààn astragale; gòngaan roue; gaan glù trace $\square$ è sóó gaan glù yà il a vu la trace d'un vélo 
gààngáán $\boldsymbol{n}$ cloche, gong [dans cette fonction on utilise souvent n'importe quel objet métalique, par example, des jantes des vieilles voitures] $\square$ ò gààngáán de bhéć lèglizì $m \varepsilon \varepsilon m u$ $\varepsilon$ nu leglizi fálà dimànsí ma on frappe le gong, et les paroissiens viennent à l'église le dimanche

gààngáán $\boldsymbol{n}$ perroquet [la peste des champs de sorgo, maïs etc.]

gaanlè v 1) vt cacher (de - léć) 2) vr se cacher $\square$ nc̀ mu ò gàànnà kpe ć lâ les enfants se sont cachés sous la chaise

gaanma <gaan+ma> $a d v$ à pied $\square$ yàa gaanma il est à pied

gaanyre $r n$ cachette

gad $\boldsymbol{\varepsilon} \mathbf{m i}<\mathrm{ga}+\mathrm{d} \boldsymbol{\varepsilon}+\mathrm{mi}>\boldsymbol{n}$ personne malade

gàfé, kàfé $<F r$. café> $\boldsymbol{n}$ café $\diamond$ gàfé yí café (boisson); gàfé diin caféier

gàgú adv gban (ethnie, langue) จ gàgúmi Gban (représentant de ce groupe ethnique)

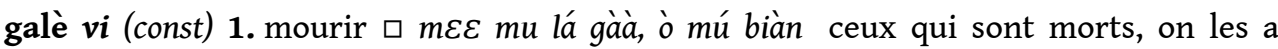
enterrés 2. sécher (des plantes) 3. être paralysé (des parties du corps) $\square$ à gbe gàà son bras est paralysé

gálíí $<$ Fr. gare $>\boldsymbol{n}$ gare

gàmà $\boldsymbol{n}$ sauterelle $\diamond$ maanc̀ gàmà grande sauterelle volante $\square$ Syn. banc̀ gbàkpu

gàmà bò móóne $\boldsymbol{n}$ grillon (mimétique au feuillage vert)

gàní $\boldsymbol{n}$ scorpion

gàní ygblokpápìtì $\boldsymbol{n}$ perce-oreille

ganyan $\boldsymbol{n}$ piège (aussi en métal) $\diamond$ ganyan lìipe appât ; ganyan lègbaanle tendre un piège

gáy onomat gan (le cri du oiseau bagbezu)

gaygle $n$ Numida meleagris pintade

gàsì <Dioula> $\boldsymbol{n}$ respect, estime $\diamond$ gàsì kpále respecter (qqn - lèc̀) $\square$ ý gàsì kpa dosò mu lè̀े j'ai du respect pour les chasseurs ; gàsi do a ma grâce à lui

gàtoò <Fr. gâteau> $\boldsymbol{n}$ gâteau

gàzè̀े $\boldsymbol{n}$ danse (type de)

gèé $a d v$ comme ça, de cette façon-ci, ainsi $\square$ à kle ò kle gbeya gbeغ̀n gèé fais comme ils le font

gele $\boldsymbol{v}$ (const) 1 . s'en aller, partir $\nabla$ gele à lé emporter, partir avec $\square$ è sJ gele án létré é lé

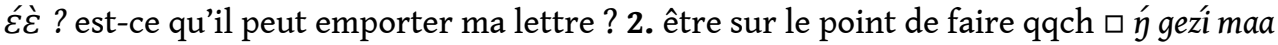
kúnle je vais attraper un poulet

genu $n$ enfant qui ressemble à un autre enfant de la même famille qui est mort [s'utilise aussi comme nom propre]

gesè $\boldsymbol{n}$ fil (fait du coton filé manuellement)

geza rn départ $\square$ Màní á geza pià ý ni Mani m'a annoncé son départ

gè $a d v$ 1. ici (proche au locuteur) $\diamond$ ká ba gè chez vous ici 2. machin (remplace un mot oublié) $\square$ ké wóo, gè $m u$ ć púclé, sàyaàn $m u$ ć et ils, les machins... ils ont surgi, les assaillants

gen $\boldsymbol{n}$ melon $\square$ è gen zikààn il a coupé la queue du melon

giglin adv ideo titubant 
giń $\boldsymbol{n}$ Myrianthus arboreus grand wounian, arbre à pain indigène (arbre)

glà $n$ 1. indigo [arbrisseau duquel on extrait une matière colorante de couleur bleue] $\diamond$ glà bò̀̀ bosquet d'indigo 2. indigo (matiére colorante) $\diamond$ glàba so pagne indigo

glaan $n$ veuvage $\diamond$ glaanle veuve [après la mort du mari la veuve ne sort pas de la maison pendant 7 jours; le mariage préférable pour la veuve est celui avec le frère cadet du mari] ; à na glaanle sa veuve; glaan sàa temps de deuil $\square$ yòo glaan sàa yí elle est en deuil ; glaanya veuvage

glaán $\boldsymbol{r n}$ cadeau (envoyé par qqn) $\diamond$ wi glaán « cadeau sans timbre " [viande enveloppée dans une feuille au champ et envoyée à qqn au village]

glàànle $v$ 1) vt 1. tirer, entraîner $\square$ ý wáa fétí ta bhlà É glàanzí bhle ma j'entraîne le mouton de notre fête par la corde 2. arracher (les main) $\square$ fàanníi $\varepsilon$ bòt ò $\varepsilon$ glàanlà gblàṅ̀ $\varepsilon$ kóó le voleur a arraché le sac des mains de la jeune fille 2) $r v$ se traîner $\diamond$ glàànle à zru ta ramper; glàànle à zi ta ramper sur les fesses 3) vi se précipiter, aller (précipitemment)

glawò, glaò $n$ Hyaena striata hyène $\diamond$ glawò klogbènè l'hyène est une grosse bête glè $\boldsymbol{n}$ grelot, clochette (un instrument musical et une décoration) $\square m \varepsilon \varepsilon$ É glè wézí l'homme est en train de sonner à la clochette $\checkmark$ gaanma gle grelot de pied

glèń $\boldsymbol{r n}$ gratin (partie d'un mets qui reste attachée au fond d'un plat, d'une casserole) $\diamond$ bàá glè gratin de riz

glèngbààn $\boldsymbol{n}$ arc-en-ciel $\square$ glèngbààn ć káàn l'arc-en-ciel est apparu glèpey $r n$ aine

glèwààn, grèwààn $\boldsymbol{n}$ hévéa (sauvage) [dont la sève sert de colle]

gligata adv ideo gros (corde, liane) $\square$ bhegwlesnya bhle ò gligata, yaa mon takánmi la corde de l'amitié est grosse, mais elle est facile à rompre

glii, grii $\boldsymbol{n}$ (contr) chose petite et ronde $\square$ yò griinغ̀ gèe c'est une chose ronde et petite comme ça (pièce de monnaie)

glinle vt 1. regarder 2. chercher $\square$ è ye gliyzí il cherche du travail $\diamond$ lìi glinle provoquer

glòló $n$ Dictyoptera cafard

glòló tén $\boldsymbol{n}$ coléoptère (esp.)

glò $\boldsymbol{n}$ cerf blanc [antilope menue avec des cornes courtes et aigues]

glò̀̀ adj 1. dur (rigide) $\diamond$ kóó glò̀̀ les mains dures (d’une personne avare) $\square$ le É kóó ò églj̀̀ lé cette femme est avare ; zru glò̀̀ courageux, impitoyable; wèle églòjlé sonner fort 2. important $\square$ mú a sóoń glòj̀ gbú é yaga bhé lè bhe c’étaient leurs trois totems les plus importants

glòòle $v t$ frotter, triturer, repasser $\square$ lenc̀ ć yòo blè púi glòj̀zí zièn kle pe lé la femme est en train de triturer des arachides pour la sauce $\diamond$ goodo piibh $\varepsilon$ fer à repasser

glon $n$ saignement du nez $\square$ glon kààn à yin zi il saigne du nez

glòn, glò̀̀n $\boldsymbol{r n}$ sorte, façon $\square$ waa bhé wi glò̀̀n $m u$ léċ bhle on ne mange pas des animaux de cette sorte $\square$ ò so mo kpén glòn yímálé quel que soit l'homme, on peut le comprendre

glù, glùù $\boldsymbol{n}$ trou, creux 口 yrìmà kpòkpò glù kan yrì ma le pic creuse des trous dans le bois $\diamond$ gboglù toilettes, latrine 

une ferme) tourne

gluy-gluy adv ideo très profond

glwáan, glóan, góna $\boldsymbol{n}$ homme, mâle, garçon (Iv.) $\diamond$ bole glwáan mu ba devenir un homme adulte (après des rites d'initiation) ; glwáanwecle coureuse d'hommes; le glin glwáan coureur de femmes ; glwáandokle une vièrge

glwغ̀, glò $\mathbf{n}$ Crocodilus niloticus crocodile $\diamond$ gwlè gbóngònć grand lézard terrestre

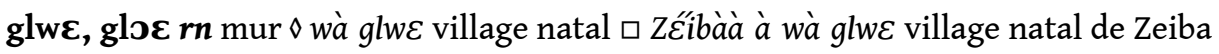

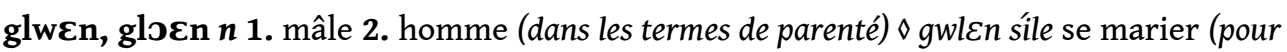

glwenna, gloعnna loc mariée, au foyer $\diamond$ yaale gwlenna se marier (pour les femmes)

glwennaya, gloعnnaya $\boldsymbol{n}$ mariage (des femmes; la vie conjugale)

gòfén $n$ flûte, harmonica

gole $v$ (const) 1) vi quitter (une place - ta) $\square$ ýgòa án wà $\varepsilon$ ta j'ai quité mon village $\diamond$ gole a yi se sauver de qqch $\square m \varepsilon \varepsilon$ ć gòa ga é yí nivakini kóó l'homme s'est sauvé de la maladie grâce à la nivaquine 2) $v t$ 1. enlever $\square$ lenc̀ $\varepsilon$ yaan $\varepsilon$ gòa tásá $\varepsilon$ yí la femme a pris l'igname de la cuvette $\diamond$ magole nettoyer; lèmagole essuyer la bouche; gbàbhe gole castrer 2. récolter (recueillir les produits de la terre) ๖ yáán go connaître, comprendre

gòmà $\boldsymbol{n}$ creux (en bas d'un arbre, termitière), grotte [souvent, la place où on adore les fétiches] 口 ò yo gba wáátí ké ba vle gòmà yí souvent on adore des fétiches dans la grotte

gòy $\boldsymbol{n}$ voiture $\square$ mí án gòn $\varepsilon$ gwân j'ai vendu ma voiture $\diamond$ gònsrá véhicule, moyen de transport ; $m \varepsilon \varepsilon$ sí gònnć taxi-brousse (Afr.)

gongoy adv ideo superbe

gòynréćmi <gòy+nréć+mi> $\boldsymbol{n}$ chauffeur, conducteur

Gòò bo mle $n$ mars (le mois où l'on plante le maïs)

goo $\boldsymbol{n}$ kola, cola [noix qui contient des alcaloïdes stimulants, utilisée dans la divination et dans de nombeux rites] $\square$ ké gòo é gbààmadwà wà pe é táà jeli sónsízíi si les noix de kola tombent «sur le dos", on dit que les esprits de tes ancêtres sont contents $\diamond$ goo diin kolatier ; goo bhe ple deux colas

gòò $n$ maïs $\diamond$ gòo yrí épi de maïs ; gòo diin pied de maïs $\square$ gààngáan mu ć yaàle ò gòo diin $\varepsilon$ yí les perroquets sont perchés sur les tiges de maïs

gooba rn surveillance $\diamond$ à gooba yaàle garder qqch, protéger qqn, surveiller; klele à gooba surveiller ; goobami gardien

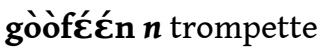

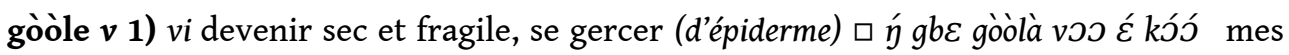
mains sont gercées par l'harmattan 2) $v t$ faire sec et fragile $\square$ vว ý gbe goo l'harmattan fait mes mains sèches et squameuses

go $\boldsymbol{n}$ brassoir (bâtonnet en bois pour malaxer la purée, la sauce) $\diamond$ go dwày kpéé mon ventre

gò $n$ Pan troglodytes chimpanzé (grand singe)

goo $\boldsymbol{n}$ queue (Afr.) (queue de cheval fixé au manche en bois ou en métal) [on estime qu'elle possède une force magique; elle est utilisée dans la danse, surtout celle des filles qui ont passé l'initiation ; elle peut être placée dans le le cercueil d'un mort]

goo yri $\boldsymbol{n}$ Terminalia glaucescens arbre (esp.) 

les fruits] des proverbes

gòkún $\boldsymbol{n}$ épée

gòòn diy $\boldsymbol{n}$ Borassus aethiopum rônier, palmier dattier [seulement des enfants en mangent

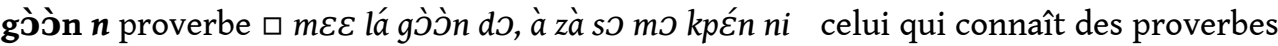
est très respecté par tout le monde $\diamond$ gòòn bole parler en utilisant des proverbes, dire

goon $n$ rocher, montagne $\diamond$ vlegJon rocher, montagne ; yi goJn berge, rive; gJonnè petite montagne ; génie de la montagne ; goon gbamu adorateurs de la montagne

goondo piibh $\varepsilon<\mathrm{g} J 0 \mathrm{n}+\mathrm{d} 0+$ pii + bh $\varepsilon>\boldsymbol{n}$ fer à repasser

goondole $v t$ repasser (habits)

gòlí $\boldsymbol{n}$ 1. argent $\square$ ké ǵ gòli náa é ni, é pebhle lo si je te donne de l'argent, tu achètes à manger $\diamond$ gòlibhe monnaie; gòli bòtòné porte-monnaie (traditionnel); gòli pú monnaie de nickel 2. cinq francs CFA $\diamond$ gòli kèmèdo cinq cents francs (lit. cent) [quand on compte l'argent, le numéral désigne un nombre qui est cinq fois plus élevé]

gon, goy $n$ partie, côté $\square$ án $p \varepsilon$ gon do na! donne-moi ma part! $\square$ f́́ $\varepsilon$ gon do klàláá un côté de la maison s'est renversé $\diamond$ gon do un côté

gònò voir gòylò faucille

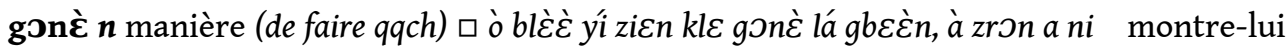
comment on fait la sauce arachide

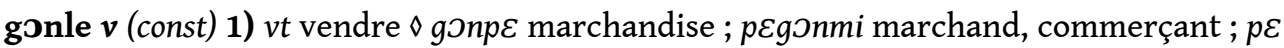
gonleya commerce 2) vi être en vente

gonwi $<$ gon $+w i>n$ viande pour la vente

gòylò, gònò $\boldsymbol{n}$ faucille $\square$ ò mlò kan gòylò lé on coupe le riz avec la faucille

grع $\boldsymbol{n}$ gueré (ethnie, langue) $\diamond$ gremi Gueré (représentant de cette ethnie)

gruu adv ideo en grand cercle

Gù do mle $\boldsymbol{n}$ août (le mois de la disette)

Gù $n$ 1. Gou (masque emprunté à l'ethnie wan) [avec une large bouche ouverte par laquelle il voit; il porte un jupon fait de liane et une peau d'animal sur le dos; il se lupe le dos avec un bâton, et l'assembée crie autour de lui] 2. danse du masque Gou $\diamond$ Gù tánle pratiquer la danse du masque Gou

gu $\boldsymbol{n}$ chat noir (sauvage) [il est interdit de le consommer pour les clans qui ont la panthère ou le lion comme totem] $\square$ gu ò bòn yí sroónz̀ wi lé «gou » est un chat sauvage

gù $\boldsymbol{n}$ famine, disette $\square$ ké gù dwà mé laa é zruma pe bhle pendant la disette on ne mange pas ce qu'on veut

gù̀̀̀̀n $a d v$ ideo d'un air moqueur

gùmàsàsà $n$ géant (un des génies de brousse) [génie de brousse de grand taille, cracheur de feu, qui est capable de voler les âmes des gens; inspire une forte peur lorsque on revient de nuit du champ; est censé être à l'origine des feux lointains vus dans la brousse la nuit] $\square$ ké gùmàsàsà á líiploólà té wian si le géant ouvre la bouche, il y a un jet de feu

gùn, gùy $a d v$ immédiatement, tout de suite, vite (surtout dans les ordres) $\square$ kà ge gùy wàla

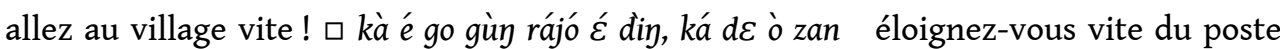
radio, votre père arrive ! 

régler les querelles éjaculer

gbàádwáá $\boldsymbol{n}$ canard qui m'a fait grandir

gùú $n$ Combretum racemosum fausse bougainville (esp. de buisson)

gwà $\boldsymbol{n}$ chevrons (du toit de la maison africaine traditionnelle) [en rafia ou en bois] $\square$ mùa ta ò gàa kpa f́́ gwà ta chez les Mwans les chevrons des toits sont couverts avec de la paille gwà $n$ Chlorophora excelsa iroko [très grand arbre, dont le bois a une grande valeur]

gwàànseńné n Acinonyx jubatus guépard

gwe adj 1. ancien (d'habitude pas par rapport à des personnes) 2. Péjor. vieille bique, vieux con $\diamond \grave{a}$ na gwe son ancienne femme (première mariée)

gwè $\boldsymbol{n}$ chat de brousse (le poil est blanc et noir)

gwèèle $\boldsymbol{v t}$ raser $\square \grave{a}$ wiy gwèèle ò il a les cheveux rasés $\diamond$ win gwèepe rasoir pour les cheveux ; lèzaa gwèè rasoir pour la barbe

gwle, gloe $\boldsymbol{n} 1$. bagarre $\diamond$ gwle danle se battre $\square$ gwláan pegée le gwle dan un homme et une femme se battent 2. guerre $\diamond$ gwle danpe arme 3. querelle, palabre, discussion, débat $\square$ wàta $m \varepsilon \varepsilon$ gwe mu éke lèyâ gwle dan zà yí les anciens du village se sont réunis pour

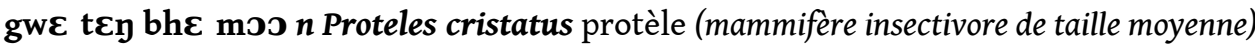

gwElóon $\boldsymbol{n}$ lièvre (esp.)

gwÈnย̀ $n$ petite houe

gwÈsé n Bitis nasicornis, Bitis gabonica vipère rhinocéros, vipère du Gabon

\section{Gb - gb, comme gbà, gbaa, gbàádwáá}

gba $\boldsymbol{n}$ 1. offrande, don (à l'église) 2. amende [le chef du village et son administration infligent une amende à ceux qui violent les lois du village] 口 ké é fàanwòà wàla, ò gba gbènnc̀ bàláá é ta si tu voles au village, on t'inflige une grosse amende

gba $\boldsymbol{n}$ champ, jardin $\diamond$ gbabhe terrain de champ [la brousse est défrichée, brûlée et nettoyée et le terrain est prêt pour la culture] ; gbakpami cultivateur ; zièn gba potager

gbà $r n$ pénis $\diamond$ gbà kpu kán wlanc̀ couteau pour la circoncision; gbà bhe gland (Anat.); gbà cíc; gbà frò testicules; gbà pínàle avoir une érection; gbà yí sperme; gbà yí kláále

gbaa $\boldsymbol{r n}$ cadavre $\diamond$ gbaama so pagne pour couvrir le cadavre [dur et noir, fait de coton]; gbaa wla... yì s'engourdir $\square$ gbaa wlàà ý gaan yì j'ai la jambe engourdie

gbààkóó $\boldsymbol{n}$ célibataire $\square$ Syn. leklezan

gbààma loc $r n$ à la renverse $\square$ ý yílàle ò ǵ gbààma je suis couché à la renverse $\diamond$ gbààma dole être couché à la renverse (sur le dos face en l'air)

gbaan $\boldsymbol{n}$ 1. babouin [peste des champs de maïs; est consideré comestible] 2. Babouin (masque et danse) [pendant la danse le masque imite les manières des babouins]

gbàan $\boldsymbol{n}$ chien [ne sont pas mangés par les Mwans, à la différence de certains groups éthniques voisins] $\diamond$ widegbàan chien de chasse

gbaand $\varepsilon v 1$. vi grandir 2. $v t$ faire grandir $\square$ yaan pon ý gbaandià c'est le foutou igname

gbàànè <gbàà+nè> voir bhenè brother-in-law 
gbààngàà $n$ poisson (esp. : poisson d'eau douce; ces nageoires peuvent produire des piqûres douloureuses)

gbaanle $v$ 1) vt enfoncer, piquer $\square$ yri gbaan tré yí enfonce le piquet dans le sol $\diamond$ yrì gbaanle piquet $\square$ mí ý gbe gbàànlà à mlè líi j'ai mis mon doigt sur son front; gbaanle à kpó ta marcher à quatre pattes 2) vi courir après, marcher après $(q q n-z i) \square \diamond n \dot{\varepsilon} m u$ gbaan ò nEE mu zi táń les enfants suivent toujours leurs mères

gbààyglò $n$ vallée

gbàbú $<$ Dioula $>\boldsymbol{n}$ cuisine

gbàgami <gbà+ga+mi> $\boldsymbol{n}$ homme impuissant [insulte pour un homme qui n'a pas d'enfants]

gbàgba $n$ masque (en général), l'homme qui porte un masque $\diamond$ gbàgba ýgbhlo masque (objet)

gbáká <Dioula> $\boldsymbol{n}$ taxi-minibus (à itinéraire fixe)

gbàkóóya <gbàkóó+ya> $\boldsymbol{n}$ statut du non-marié

gbàkpú $n$ 1. Zonocerus orthoptera sauterelle (peste des champs) 2. Vulg. pénis coupé (en parlant des Dioula qui pratiquent la circoncision)

gbale $v t$ 1. se partager avec, partager (donner une partie de ce qu'on a) 2. sacrifier, adorer (un fétiche)

gban $a d v$ déjà, tout à l'heure, juste maintenant $\square$ Táátoo gbèn ć yâ gban ganyan É líi Tato a juste vu l'écureuil dans le piège

gbane $\boldsymbol{n}$ chauve-souris [comestible dans les sauces; autrefois on les attrapait en mettant de la sève d'hévéa sauvage sur l'arbre où ils vivent]

gbanegbelàà <gban-gbe-làà> $\boldsymbol{n}$ parapluie $\square$ la ò banzí bhémalè Màlí gbanegbelàà ć sía il pleut, c'est pourquoi Marie a pris une parapluie $\diamond$ gbanegbelàanć petit parapluie

gbay $\boldsymbol{n}$ claie (pour sécher les plantes)

gbàybháálú $\boldsymbol{n}$ Ardea cinerea héron cendré

gbaybhle $\boldsymbol{n}$ cravache [faite en corde de rafia, on l'utilise pendant les danses pour dispercer les gens afin de frayer le passage au masque]

gbàybhlè $\boldsymbol{n}$ rouquin [on les considère comme jolis, à la différence des albinos]

gbànglànbàá $\boldsymbol{n}$ bouillie de banane et d'arachide (mélange de bouillie de banane et d'arachide grillée et broyée)

gbaò $\boldsymbol{n}$ Viverra civetta civette (petit carnassier)

gbào onomat (bruit de coup de fusil)

gbàtó $\boldsymbol{n}$ sénoufo (ethnie, langue) $\diamond$ gbàtómi Sénoufo (représentant de cette ethnie)

gbawole vt payer (à - ni ; pour - ma; pour faire qqch - za yí) $\square$ è gòli wáádo gba wòa yaa sosràmi ni dunè dre srá za ýi elle a payé 5000 francs à son tailleur pour la couture de sa nouvelle robe $\square$ Màní wáá vu gba wòa sòo $\varepsilon$ ma Mani a payé 50000 francs pour le vélo

gbe rn 1. fils 2. fils (forme d'adresse à un homme de la plus jeune génération, sans lien de parenté)

gbe $\boldsymbol{n}$ bras de fer $\diamond$ gbe tánle faire bras de fer $\square$ sò $m \varepsilon \varepsilon$ mu $\varepsilon$ yàa gbe tan auparavant les gens faisaient le bras de fer

gbè $\boldsymbol{n}$ cri (seulement d’homme) $\diamond$ gbè kánle crier, exclamer 

gibier] kola] semaine sont noires épineux] $60 \mathrm{~cm}$.]

gbee $\boldsymbol{n}$ piège (sorte de) [fait d'un caillou fixé sur un tronc vertical; le caillou tombe sur le

gbèè $r n$ nageoire $\diamond$ gbèe dole faire malin, prendre des airs $\square$ yà gbèe dozí á ma il prend des airs d'importance

gbèèsáàn $n$ guêpe $\square$ gbèesáàn $m \varepsilon \varepsilon$ paa la guêpe pique l'homme

gbèlúú adv ideo en biais, du coin de l'œil (regarder)

gbèy $\boldsymbol{n}$ cour (à l'intérieur de la clôture) $\diamond$ wláma gbèy cour commune (Afr.)

gbètégbèné $\boldsymbol{n}$ génie de brousse [de petite taille, avec la peau jaunâtre et des cheveux longs, ils apparaissent pendant la nuit]

gbe $n$ 1. $r n$ bras, patte avant (d'animal, d'insecte), aile (d'oiseau) $\diamond$ gbesrò ongle, griffe, serre; gbenc̀ bhe doigt; gbesàa paume (tendue); gbelàà paume (non tendue); gbekpùn

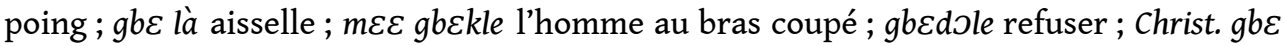
kpále wì̀ imposer les mains (pour faire un miracle, etc.); à gbe zi laa ò il est maladroit

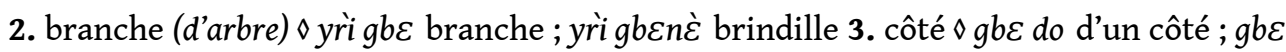

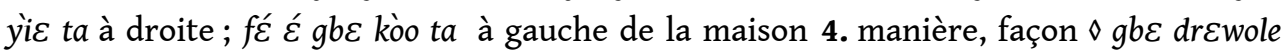
faire de nouveau ; án kle gbe drewoà je l'ai fait de nouveau ; ye gbe façon de voir

gb̀̀ $\boldsymbol{n}$ punaise (de lit)

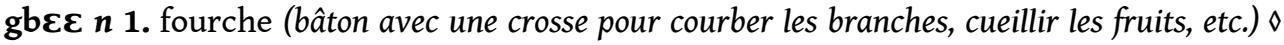

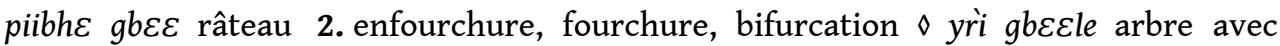
bifurcation ; zi gbec carrefour, bifurcation de route

gbèc̀fì $\boldsymbol{n}$ perles (parure traditionelle)

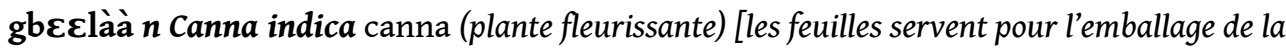

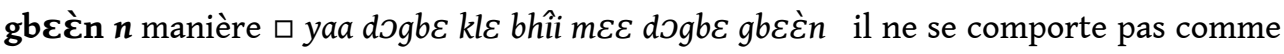
un être humain $\square$ Màní kpále ò Zàan yí bhîi à gbú gbeغ̀n Mani a confiance en Jean comme en lui-même $\diamond$ a gbec̀n convenablement, comme il faut $\square$ à bhle a gbec̀n! mange

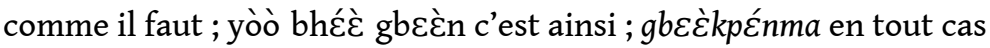

gbèz̀yi $\boldsymbol{n}$ semaine $\diamond$ gbèżyi lá zan bhe la semaine prochaine; gbèżyi

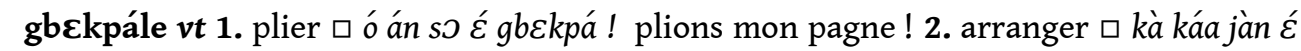
gbekpá bhéc̀ ká Wàànbhaa púbo arrangez vos affaires et remerciez Dieu

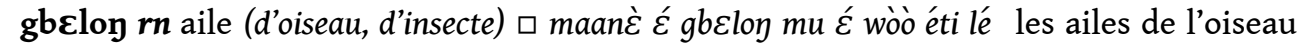

gbemadiní $n$ Malapterurus electricus poisson-chat électrique, silure électrique,

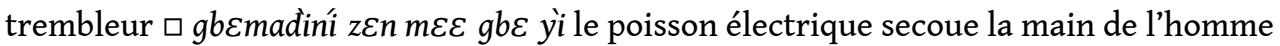

gbèn $n$ Paraxerus palliatus rat palmiste [petit rongeur proche de l'écureuil, aux poils durs et

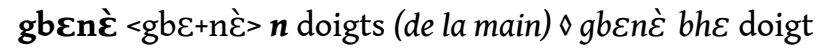

gbèngbènnì $n$ Vulpes famelicua, Fennec Chama renard, fennec [ressemblant à un petit renard, pourvu de longues oreilles et habitant au Sahara et au sud du Sahara; mesure environ 
gbenle vt attendre, guetter $\square$ sroónè moว gben glù ć líi le chat attend la souris près du trou $\square$ góo ý nój gbenzí zi ć líi j’attends mon mari sur la route $\diamond$ zi gben yre barrage routier

gbènnè adj grand $\diamond$ jàn gbènnc̀ parole sérieuse

gbenta loc en voyage $\diamond \grave{e}$ jà gbenta il est allé en voyage ; gbenta ge mu voyageurs

gbèntèn adj grand

gbèntغ̀nné $\boldsymbol{n}$ Funisciurus pyrrhopus écureuil à pattes rouges, écureuil rouge

gbéy $\boldsymbol{n}$ enclume $\square$ tònmi piibhe pii gbéy ta le forgeron bat le fer sur l'enclume

gbepààloy $r n$ épaule $\square$ bhle do ò é gbepààlon ta il y a une termite sur ton épaule

gbéwóle adj clair, juste, honnête (sans défaut moral) $\diamond$ jàn gbéwóle parole lucide

gbewole vi refuser

gbéwóleya $\boldsymbol{n}$ justesse, honnêteté (absence de défauts moraux)

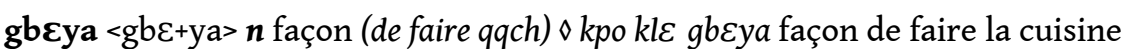

gbìi adv ideo brrr ! (bruit d'oiseaux qui s'envolent)

gbiin, gbiy $\boldsymbol{n}$ rêve $\diamond$ gbindzle rêver $\square$ ý gbiydia pò̀mi lé j'ai rêvé d'untel $\square$ ké $m \varepsilon \varepsilon$ gbindià ké yò wi bhlezí, bhé kle sróónníi mu ò à zi si quelqu'un rêve d'être en train de manger de la viande, cela veut dire qu'il est poursuivi par des sorciers $\diamond$ gbigla en rêve $\square$ mí ý ná yâa gbiy la j'ai vu ma femme en rêve

gbìnle $\boldsymbol{v t}$ (const) chasser (éloigner) $\square$ sranè mu gbìy za yí, è màfá wía laanima pour chasser les tisserins il a tiré en l'air

gbiynàle vi (const) rugir (lion), gronder (tonnerre), râler (personne malade) $\square$ gblàn gbinnáá le tonnerre a grondé

gbla, gblaa $n$ hernie

gblà $\boldsymbol{n}$ jeune fille (pas mariée, qui n'a pas d'enfant) จ lù gblà jeune fille (non-mariée)

gblàànle $v 1$. vi avoir peur, respecter (qqn - léć) $\square$ le mu gblaan à nò léć les femmes ont peur de leurs maris $\square$ ǵ gblaan dosò mu ma j'ai du respect pour les chasseurs 2. se méfier $($ de $q q n-m a) \square$ sò $m \varepsilon \varepsilon$ tí yàa gblaan tùàbù $m u$ ma auparavant les Africains se méfiaient des Blancs

gblà-gblà-gblà onomatop boum (bruit des bottes)

gblàn $n$ peur $\diamond$ gblàndole faire peur à $\square$ ò $n \varepsilon \grave{~ m u ~ g b l a ̀ n ~ y i ́ d o ~ b i ̀ z i ~ o n ~ f a i t ~ p e u r ~ a u x ~ e n f a n t s ~}$ la nuit ; gblàn gole enlever la peur $\square$ ké è gblàn gwà ý yí, ý nu gele Dalowa s'il dissipe ma peur, j'irai à Daloa

gblan, gblaan, gbana adj (contr) 1. grand, gros 2. important 3. Fig. adulte, vieux (respectueusement) $\diamond n \dot{\varepsilon}$ gblannغ̀ vieillard; le gblannc̀ vieille; $n \varepsilon \varepsilon$ gblan grand-mère

gblànluu $n$ éponge (traditionnelle, en fibres de bois)

gblanya <gblan+ya> $\boldsymbol{n}$ 1. orgueil $\square$ Setrán a gblanya ma Wàànbhaa á gbiàn à cause de l'orgueil de Satan, Dieu l'a rejeté 2 . vieillesse $\square$ lenc̀ $\varepsilon$ le gblanya dià la vieille femme a beaucoup vieilli

gblày $\boldsymbol{n}$ tonnerre $\diamond$ gblày $p \varepsilon \varepsilon$ il tonne; gbláy bhóbhó éclair

gblasata adv ideo haut et élancé (homme, arbre) Fig., Péjor. 
gblè $\boldsymbol{n}$ baobab [fruits sont mangeables] $\diamond$ gble bhe fruit de baobab, pain de singe; gble diin le baobab

gblèbà <Dioula> $\boldsymbol{n}$ chemin, route (grande), rue $\diamond$ gblèbà gbèntèn bloole ple É ta route à deux voies

gblè̀le $v t$ 1. éduquer, élever 2. se charger de l'éducation d'un enfant, éduquer (un enfant d'une autre famille) $\square$ è twénc̀ É gblèèà il a éduqué l'enfant orphelin 3. élever, domestiquer (animal, oiseau) $\square$ ý bhle maa do nàa Màní ni bhéċ è maa É gblèèà j'ai donné le poulet à Mani pour manger, et il l'a apprivoisé

gblèma kúùkúù $\boldsymbol{n}$ Polistes Vespidae, Hymenoptera frelon

gblèn $n$ 1. odeur $\square$ pebhle É gblèn nra l'odeur de la nourriture est bonne 2. matière en suspension $\diamond$ té gblèn fumée ; bwe gblèn poussière dans l'air

gblèý n Annona squamosa anone, pomme cannelle (arbuste) [arbre de la famille des anonacées, poussant dans les régions équatoriales et faisant penser au pommier; fruit charnu aussi appelé pomme cannelle, sucré, parfumé, issu de cet arbre]

gblè̀ pú $\boldsymbol{n}$ Chrysichthys nigrodigitatus mâchoiron (poisson d'eau douce)

gblè̀ pú tén $\boldsymbol{n}$ Clarotes laticeps clarote (poisson)

gbli bhaa le gwlećn $n$ Khaya grandifoliola acajou d'Afrique, acajou blanc

gbliy $n$ Python sebae, Eryx thebaicus python africain

gblò $\boldsymbol{n}$ ténèbres, obscurité $\square$ gblo wlàa les ténèbres sont tombées

gblò $r n$ cuisse

gblúy $\boldsymbol{n}$ pilori, sabots en bois (pour les fous) [cadre en bois dur, avec des trous dans lesquels on met les pieds des fous]

gbo $n$ 1. $r n$ excrément (des humains, des animaux) $\diamond$ gbo glù toilettes; gbo kli anus; gbowole déféquer 2. déchet 3. sécrétion solide $\diamond$ trón gbo cérumen; yrغ̀ gbo chassie, caca des yeux (Iv.)

gbòflàà $\boldsymbol{n}$ caillou pour foyer (de couleur marron)

gboglù $r n$ toilettes, latrine, WC

gbòý $\boldsymbol{n}$ toupie (fleur de fromageur qui sert de jouet aux enfants) $\diamond$ gbòn bhóle tourner la toupie

gbóngbó $n$ seau (métallique)

gbòò $n$ Phacochoerus africanus phacochère [cochon sauvage d'Afrique possédant deux défenses recourbées]

gbòò $\boldsymbol{n}$ son, balle $\square$ \mlò gbòo le son du riz ; yri gbòo sciure de bois

gboo $\boldsymbol{r n}$ corps $₫ m \varepsilon \varepsilon$ gboo corps de personne; yri gboo tronc (d'un arbre tombé); vì gboo fromager tombé $\square$ è maa gboo do bhlà il a mangé le poulet entier

gbòogbe $n$ Heliosciurus gambianus écureuil (esp.)

gbòòle $v$ 1) vi être paralysé $\diamond$ è gbòò̀ il est paralysé 2) $v t$ paralyser $\square g a m \varepsilon \varepsilon$ gboo la maladie peut paralyser une personne

gboóóó, gbóló $n$ serrure $\diamond$ gboóló bh $\varepsilon$ cadenas $\square$ ý gbe án kàyàn gbóólóbhe riì mon fils a perdu le cadenas du coffre

gbòonè n Xerus erythropus écureuil de terre [la peste des champs d'arachide] 
gbòsró $\boldsymbol{n}$ tabac (aussi la plante) $\diamond$ gbòsró míle fumer ; gbòsró wlále chiquer du tabac ; gbòsró glòle chiquer de la feuille fraîche de tabac

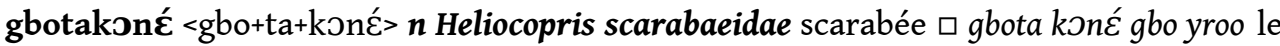
scarabée roule la boule de fumier

gbotakwé <gbo-ta-kwé> n 1. Torgos tracheliotus vautour 2. Gyps bengalensis condor africain

gbo $\boldsymbol{n}$ canari (Afr.), marmite, pot (en argile) $\diamond$ yì klá gbo canari à eau, jarre

gbò̀̀ $\boldsymbol{r n}$ ancêtre, aïeul (personne à l'origine d'une famille) $\diamond$ gbò̀̀gwláan arrière-grand père ; gbòj̀le arrière-grand-mère

gboon adj 1. long, étendu (personne, vêtement, objet, chemin) $\square$ zi É yòo égboonle le

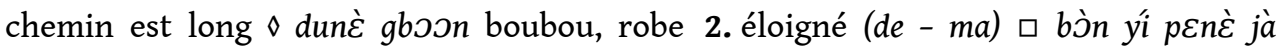
égboonle wà ć ma sánsí gblèz̀n ć kóó les esprits de brousse se sont éloignés du village à cause de l'odeur d'essence

gbògbò quant beaucoup $\square$ zààn gbògbò yàa làànć láá bhlézíi zi ć líi beaucoup de gazelles mangeaient les feuilles d'arbre sur la route $\square$ Syn. bèbè

gbòlòkó $\boldsymbol{n}$ pian (maladie de peau contagieuse, se manifestant par des lésions cutanées).

gbòlù adj terre infertile $\diamond$ le gbòlù femme après l'âge de procréation

gbon $\boldsymbol{n}$ faim $\diamond$ gbon ò $y ́ d \varepsilon z i$ j'ai faim ; gbòndemi personne affamée

gbon $r n$ intestins $\diamond$ gbonbhle intestin ; kpée gbon intestins ; maagbon spaghettis

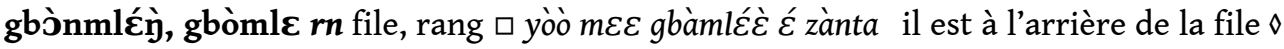
gbònmléc̀ ma par ordre ; dole éke gbònmléc̀ma disposer par ordre ; kà do éke gbònmléc̀

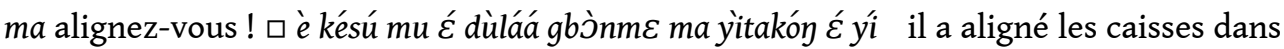
la pirogue

gbowì $\boldsymbol{n}$ lézard (venimeux)

gboyròó $\boldsymbol{n}$ Labeo senegalensis carpe d'Afrique

gbú adj même $\square$ ý gbú ò nè ć félé je vais laver le bébé moi-même $\square$ è klà mí gbú náágwlećn do ni c'était arrivé à mon propre grand frère $\square$ à gbú ò zrá gba ć yí lui-même est au champ de tabac

gbù quant moitié $\diamond$ à gbù lè c'est la moitié de qqch u blè tásá yàn do gbù demi-cuvette d'arachide $\square$ loonè bàá kòo ć bèbè bhlà, bhéć yà gbù tòa le lièvre a mangé beaucoup de riz, et il en a laissé la moitié

\section{$\mathrm{J}$ - j, comme jàrà, jàwà, jह̀kwàjó}

jàmànà <Dioula> $\boldsymbol{n}$ pays $\square$ MEE ti jàmànà l'Afrique $\square$ Syn. klén

jàmàtìi <Dioula> $\boldsymbol{n}$ chef de canton [chef du group éthnique sur lequel l'administration coloniale s'appuyait]

jàn, jan $n$ parole $\diamond$ jànwóle parler $\square$ ò Zèzù là a jàn wózíi c'est de Jesus qu'on parle ; jànwomi narrateur ; jànma táâné devinette

3 jàrà, jààrà, jlà $\boldsymbol{n}$ lion $\diamond$ jàràmá jàrà chef des lions (animal mythique qui est plus fort que le lion), « tigre »

jàrànàmà $<$ Dioula $>\boldsymbol{n}$ Christ. enfer $\diamond$ jàrànàmà té feu d'enfer 
795 jásá, jósó <Dioula> $\boldsymbol{n}$ palissade, clôture (en bambou, en roseau, en paille)

796 jàwà $<$ Dioula $>\boldsymbol{n}$ oignon

797 jàwànì $<$ Dioula $>\boldsymbol{n}$ henné [les femmes l'utilisent pour se teindre les pieds et les ongles selon les pratiques des femmes dioulas]

798 jàwòflá, jààflá <Dioula> $\boldsymbol{n}$ oignon $\square$ jàwòflá gblèn $m \varepsilon \varepsilon$ yre yí bho l'odeur de l'oignon donne des larmes aux yeux

799 jèkwàjó < Gouro> $\boldsymbol{n}$ paludisme

800 jèkwàjó diin $\boldsymbol{n}$ Newbouldia laevis hysope africaine

801 jè̀kwàjód $\boldsymbol{\varepsilon} \mathbf{m i}<\mathrm{j} \dot{k} k w a ̀ j o ́+d \varepsilon+m i>n$ paludéen

802 jesìn <Gouro> $\boldsymbol{n}$ dracunculose $\diamond$ jesìíbhe Dracunculus medinensis ver de Guinée (parasite)

803 jiè, jièè $\boldsymbol{r n}$ grand-mère (paternelle et maternelle)

804 Jì , Jì $\boldsymbol{n} \boldsymbol{n}$ 1. Djé (masque) [le plus dangereux masque sacré qui sort pendant les funérailles des hommes iniciés; les femmes ne doivent pas le voir: la femme, qui a vu ce masque, meurt. C'est le seul masque qui est mis non dans le village, mais dans la forêt. Celui qui porte ce masque est capable de danser dans le feu et sur des charbons ardents.]

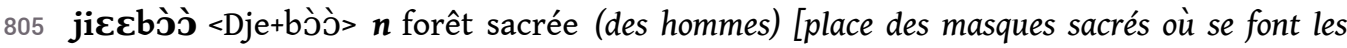
cérémonies d'intiation des garçons]

806 jiclii $n$ Animist. 1. esprit, fantôme (principalement, l'esprit d'un ancêtre) $\square$ mí ý táa jiclii yà j'ai vu l'esprit de mon grand père 2. Djé (homme en masque Djé) $\square$ ké le jeli yà è ga àma si une femme voit ce masque, elle va mourir absolument

807 jilalè vi 1. partir, disparaître $\square$ fàanníi $\varepsilon$ jilàa bhéć à gbe nri laa bole le voleur est disparu sans laisser des empreintes digitales 2. partir (pour toujours)

808 jìlòỳ $a d v$ très (noir) $\diamond$ tí j̉ilò̀y, tén jỉlòỳ noir

809 jìmàsí $<$ Fr. dimanche $>\boldsymbol{n}$ dimanche

810 Jòjàn <Dioula> $\boldsymbol{n}$ masque-géant, «Jojan » (masque échassier et sa danse)

811 jómóró $\boldsymbol{n}$ balafon [instrument musical de type du xylophone formé de lames de bois] $\diamond$ jómóró dele jouer du balafon ; jómóró deyri baguette de balafon

812 jóó $\boldsymbol{r n}$ comportement, conduite $\square$ mo kpén pegée mú jóo lè chacun a son comportement

813 jósó $n$ palissade

814 jrà $\boldsymbol{n}$ ceinture (faite de tissu ou de fil de coton; utilisée par les femmes)

815 juwìnn $\boldsymbol{n}$ hérisson

\section{K - k, comme kaa, kààlón, kaamj̀j}


káá (ká+laa) pron vous ne... pas (2 pers. pl.)

káá conj que (de proposition explicative) $\square$ Màlìi pe Zàle ni káá yà win yigo Marie demande à Zalé de la coiffer $\square$ Syn. káádoo

kàà $\boldsymbol{n}$ lime (instrument) $\square$ ò wla lèkpa kàa lé on aiguise le couteau avec la lime

kaa $n$ poisson $\diamond$ kaa wee queue de poisson $\diamond$ kaa klo écaille; kaa gàa branchies; kaa dele pêcher ; kaadzmi pêcheur

kaabhle maanc̀ <kaa+bhle+maa+nغ̀> $\boldsymbol{n}$ oiseau pêcheur

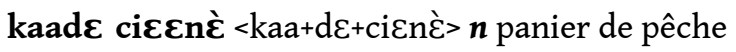

$\operatorname{kaad} \varepsilon$ maanc̀ $<\mathrm{kaa}+\mathrm{d} \varepsilon+\mathrm{maa}+\mathrm{n} \grave{\varepsilon}>\boldsymbol{n}$ oiseau pêcheur

kaagblòó $n$ Tilapia galilaea tilapia (poisson d'eau douce) $\square$ Syn. tataanغ̀

kaale manè $n$ Ardea (goliath) héron (goliath)

káále v 1) vt gratter $\square$ mí ý lèzàa káází je me gratte le menton 2) vi 1. démanger $\square$ ý yin zi ò káázii le nez me démange 2. donner démangeaison $\square$ gàà kaa la paille donne des démangeaisons

kààlóy $n$ écrevisse, crabe

káámà <Dioula> pstp à cause de, contre (avec substantif, gérondif) $\square$ ý nwâé káámà je suis venu à cause de toi.

kaamòò <Dioula kàramıgo> $\boldsymbol{n}$ marabout (enseignant dans une école coranique; l'homme savant)

káánle vi marcher (des hommes, des animaux, de transport), se promener (des hommes) $\square$ bísì mu kannáá tùàbù wà kpée dans les villes européennes circulent des bus

káárá $\boldsymbol{n}$ abri de chasseur

kaato $n$ pêche

káfé, café voir gafé café

kàkàó, kàókàó $\boldsymbol{n}$ Theobroma cacao cacaoyer, cacao (fruit et graines du cacaoyer) $\diamond$ kàkàóbhe grain de cacao

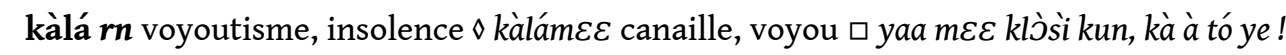
kàlá $m \varepsilon \varepsilon$ lè il ne respecte pas les gens, laissez-le ! c'est un voyou ! ; kàláya voyoutisme

káli $<$ Fr. car $>n$ bus

kámoo, ká mu pron vous (2 pers. pl,, série emphatique)

kànkàn $a d v>$ tÉn kànkàn rouge

kánle v 1) vi 1. passer $\square$ à gbe kààn kò diin ć lâ son fils est passé sous l'arbre de koo

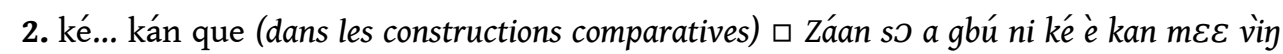
mu ta Jean s'aime plus qu'il n'aime les autres $\square$ ǵ kwé kpíinle gbènè si ké bhé kan yée ta je peux soulever un fardeau plus lourd que lui $\diamond$ kánle dàn ta dépasser la mesure (Fig.) 2) $v t$ passer à travers, traverser $\diamond$ yikánle nager ; yita kánle nager à la surface de l'eau

44 kánle $v$ 1) vt couper $\diamond$ wànyán kánle faire la cicatrice 2) vi être coupé $\square$ à gbe kpu kánle ò il a la main coupée

kánpémá $<$ Fr. campement $>\boldsymbol{n}$ campement

kányaanné $n$ Genetta sp. genette

Káygople $\boldsymbol{n}$ dimanche [jour de marché de Kongasso] 
kaykay onomatop sonnerie (de la cloche)

kari $\varepsilon<$ Fr. carrière $>\boldsymbol{n}$ loc carrière

kàsò $<J u r a<$ Fr. prison> $\boldsymbol{n}$ prison $\square$ wà bwà kàsò yí on l'a mis en prison $\diamond$ kàso fé prison

Kawaa $n$ Kaoua (masque) [appelle aux funérailles, on s'adresse à lui pour trouver le voleur]

kàyàn $<$ Dioula $>\boldsymbol{n}$ coffre (de metal)

ké 1. certain, quelconque, quelque $\square$ mángròo bèbè o é kóó, à ké ná ý ni tu as beaucoup de mangues, donne m'en une $! \nabla m \varepsilon \varepsilon$ ké quelqu'un, personne (avec négation); $p \varepsilon$ ké quelque chose, rien (avec négation); $p \varepsilon$ ké láá ò ý ma je vais bien $\square$ Syn. $p \varepsilon$ do

ké conj si, quand $\square$ ké yén dià bhí pegéé dèle nu yewole? si tu me tues, avec qui travailleras-tu?

ké prep 1. avec (comitatif) $\square$ è soófali náà do lwà ké soófâli né do il a acheté une ânesse avec un ânon 2. en num. marqueur de connexion des noms de centaines et dizaines $\square$ kemeyaga ké mia do bhe vu ta srwáa trois cent trente huit 3. partie de la construction équative ke... lé

kébhéláálè conj sinon a gbanegbelàa é sí ké bhé láále la é nu é dòj̀ bole prends le parapluie sinon la pluie te mouillera

ké pron ce

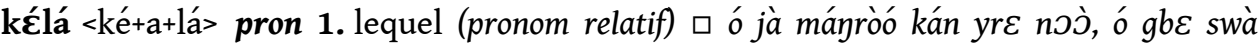
kÉmulá $m a$, mú lè gè nous sommes allés cueillir des mangues, voici celles que nous avons pu trouver 2. quel ? (pronom interrogatif) $\square$ é gwà kélá gbeغ̀n wà ह́ ta? de quelle façon as-tu quitté le village?

kélè <ké+à+lè> pron 1. avec cette chose $\square$ ké é jà nu kéle si tu viens, viens avec lui 2. c'est $\diamond$ mèle kéle ? qu'est-ce que c'est?

kèlíkèlí adv ideo à grandes enjambées

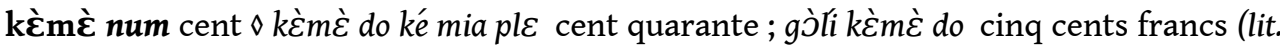
cent)

kemedomi adj centième

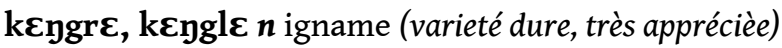

kíkí adv ideo (contr) plein (complètement) (de - kóó) ם kòo É yòo kíkí kaa É kóó le panier

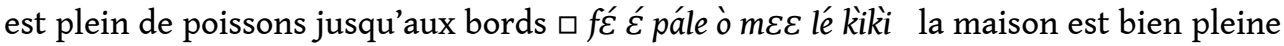
de gens

kíló $<$ Fr. kilogramme, kilomètre> $\boldsymbol{n}$ 1. kilogramme 2. kilomètre 3. balance $\diamond$ danle kíló ta monter sur la balance ; kíló gbana bascule

kítî $n$ jugement (moderne ou traditionel, effectué par le chef de village) $\diamond$ kití kánle juger $\square$ dùtìi ć Màní na a kití kánzí le chef du village est en train de juger la femme de Mani

klá $n$ Tauritragus derbianus, Alcelaphus major bubale, élan du Cap

Klàágò $\boldsymbol{n}$ vendredi

kláále $v$ 1) vi se renverser, chavirer (pirogue) $\square$ kóy ć klálàa la pirogue s’est renversée 2) $v t$ verser, mettre $\square$ mlò É klálá bàlikóy ć yí verse du riz dans le baril !

klàfà $n$ qui est confié $\diamond$ klàfà kpále donner pour garder, transmettre, confier $\square$ Zàle Sità né ć kláfa kpáa Dwàle ma Zalé a confié l'enfant de Sita à Duale

klàgbà $n$ Siphunculata 1. pou de corps $\diamond$ wiý klàgbà puce (des animaux) 2. punaise 
klàkla adv ideo vite

klàkó $\boldsymbol{n}$ petite galette ronde [fait d'un mélange de pâte de banane et de farine de manioc; frite dans l'huile de palme dans une poêle spéciale avec des cavités]

klále vi 1. mettre $\diamond$ klále à ba surprendre $\square$ ò gele wàla é kláa ý ba leur départ pour le village m'a surpris ; klále éke ba se rassembler, se rencontrer u ò klàa éke ba ye é wó za yí il se sont rassemblés pour faire le travail 2. porter $\square$ è dunc̀ gboon kla a ta táy, bhéċ è dunÈ kpeteekpe klàa à ta zòn il porte habituellement un boubou, mais aujourd'hui il a mis une chemise à manches courtes 3. ajouter $\square$ ý wE Éké klàa à ba j'y ai ajouté du sel ; tré klále couvrir de terre $\square$ sroónc̀ tré kla gbo ta le chat couvre ses excréments avec du terre

klálekéta $\boldsymbol{n}$ groupe d'assistance mutuelle (pour un travail en commun dans les champs)

klànklánn $\boldsymbol{\varepsilon} n$ moucheron

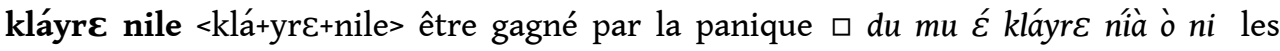
buffles se sont dispersés en panique

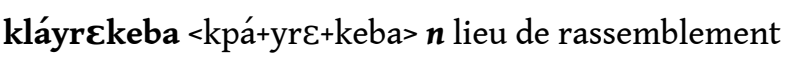

kle $\boldsymbol{n}$ message (oral, envoyé par qqn à qqn) $\diamond$ kle bole envoyer un message, faire une commission (Iv.) $\square$ ý kle bo Màní ta Zàle ni j'envoie un message à Zalé par Mani

kle part 1. sans (particule privative) $\diamond$ srònż̀ wee klee un chat sans queue; zà yaan do kle idiot 2. non (négation non verbale) $\square$ à maza ò wèc̀nmími kle gwláan do ma il a besoin d'un homme qui ne boit pas d'alcool

kléé adv fort (de sons, bruit) $\square$ gbèkánle klée des cris forts

klélé $\boldsymbol{n}$ balayures, ordures $\diamond$ klélé kláá jeter des ordures $\square$ o klélé klaláá blinta wà ma égboon lè on jette les ordures loin du village

klèdòò $n$ pie u lenc̀ É lé ma nra bhiii klèdòj̀ les lèvres de la femme sont sucrées comme celles d'une pie (elle parle beaucoup)

$\mathbf{k l}$ k̇̀ेloy $<$ Fr. clairon> $\boldsymbol{n}$ trompette

klegbeya $<\mathrm{kl} \varepsilon+\mathrm{gb} \varepsilon+\mathrm{ya}>\boldsymbol{n}$ manière, façon (de faire qqch) $\square$ yaa kpo klegbeya sa façon de faire la cuisine

klèklè $\boldsymbol{n}$ piment $\square$ klèklè sra le piment pique

klele 1) vt 1. faire, fabriquer $\square$ kpe kle ý ni fabrique-moi une chaise $\diamond$ dogbe klele se comporter 2. faire (qn faire qch) $\square$ tuàbù mu mwà mu klà silikún yewomi lé les Blancs ont contraint les Mwans aux travaux forcés 2) vi 1. être (au futur, progressif, imperatif, optatif), exister $\square$ Táatóo nu klele Yòúgò̀n gbèc̀yi lá zan bhe Tato sera à Yopougon la

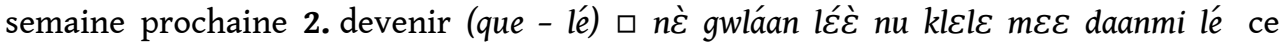
garçon sera enseignant $\square$ kle élré lé sois gentil ! $\diamond \hat{y} k$ kâ ý ta j'ai tressailli de surprise

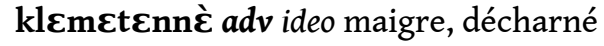

klen $a d v$ > dole klen être sur ses gardes, être vigilant

klén $\boldsymbol{n}$ excision $\diamond$ klén klele Trad. exciser $\square$ ò le mu a klén kle mwà ta les femmes étaient excisées chez les Mwans

klén $n$ pays, territoire $\square$ gwle nwà CI klén yí la guerre est venue en Côte d'Ivoire $\square$ Syn. jàmànà

klénbòò <klén+bò’̀> $\boldsymbol{n}$ forêt sacrée (où l'on fait l'excision des femmes) 
klénjèc̀ <klén+jèc̀> $\boldsymbol{n}$ association secrète des femmes [les membres fabriquent et utilisent les masques sacrés cachés des hommes] $\diamond$ klénjèc̀le membre de cette association

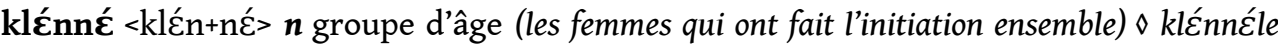
femme initiée

klènné n petit grain, granule, petit cristal

klénya $n$ santé (bonne) ৩ o klénya yí être en bonne santé ; klényá wóle état de santé $\square \grave{y}$ klényá wóle ò égbana lé je me sens bien

klèygbéjoro $n$ Bubulcus ibis héron garde-bœuf [ils accompagnent les groupes de bovins]

klèyglè $\boldsymbol{n}$ igname (sorte), klinklin (Afr.)

kli $\boldsymbol{r n}$ pied (arbre, montagne) $\square$ ýjà à kpále vle goon ć kli ma je suis allé pour le mettre au pied de la montagne

kli $\boldsymbol{n}$ amulette (en cuivre portée au reins ou en écharpe) [protection contre la maladie, particulièrement, la maladie néphrétique] bhle kli amulette au cou $\square$ è bhle kli do dwà à bhloo ma ga $\varepsilon$ léć il a mis une amulette à son cou contre la maladie

kli adv ideo ferme

klìnle $v(m o b)$ 1) vi $\mathbf{1}$. se coincer $\square$ glawò klinnà loonغ̀ glù líi l'hyène s'est coincée dans le

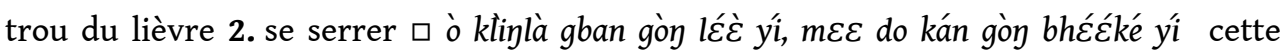
voiture est déjà remplie, il faut que quelqu'un prenne une autre voiture 3. faire (jour) $\diamond$ too klinnà il a fait jour 4. avoir des problèmes 2) vt bourrer, fourrer $\square n \varepsilon \dot{\varepsilon}$ ć lomlin klinlà bذ̀tว̀ É ýi l'enfant a bourré le sac d'oranges $\square$ ý blàko kpu kliglà bùtèli líi j'ai enfoncé le bouchon dans la bouteille

klo adj inutile, sans valeur Péjor. $\diamond m \varepsilon \varepsilon k l o$ personne qui n'est bonne à rien; peklo vieillerie (vêtement, chaussure, vaisselle)

klo $\boldsymbol{n}$ écorce (arbre), pelure (banane, igname), écaille (poisson, serpent, crocodile), carapace (tortue, pangolin), coquille (œuf) $\diamond$ klo gole enlever (écorce, pelure, écaille etc.); klo bhE bouton; klo lèbole boutonner; klo lègole déboutonner

klogbènè $n$ Potamochoerus porcus potamochère

kloya $<$ klo+ya $>\boldsymbol{n}$ sottise, bêtise

klo $\boldsymbol{n}$ >> bhl⿷ klo rouleau (de corde, de liane grosse et sèche)

klóo $\boldsymbol{n}$ maître $\square$ klóo ò bhéċ lu ò il y a des maîtres et il y a des esclaves

klòy $\boldsymbol{n}$ puits [un trou d'eau couvert ou souvent non-couvert, on y puise de l'eau] $\diamond$ klòy glù puits

klòsì <Dioula> $\boldsymbol{n}$ attention, respect $\diamond$ klòsì kúnle remarquer $\square$ yaa zà ké do kpó klòsì kun à diy il ne remarque rien autour de lui; respecter $\square m \varepsilon \varepsilon$ é léćmi klòsi kun il faut respecter les plus âgés

klù $n$ hibou

klu $\boldsymbol{r n}$ race, descendant, rejeton $\diamond$ yaan klu rejetons d'un tubercule d'igname

klùbhòbhò $n$ hibou [selon les croyances, des sorciers se transforment en hibou pour voler les âmes des gens]

klùgbé $\boldsymbol{n}$ souris (de la maison)

klúklú adv ideo puant, repoussant (d’une forte puanteur) $\square$ wi vEn dwà klúklú la viande sent trop mauvais 
klùné n pygmée, nain Péjor. courtaud (personne de petite taille)

klùta $n$ cour, tribunal $\square m \varepsilon \varepsilon$ mu lá flámu a dri É fáánlà bhe, ò jà ò le klùta ceux qui ont volé le boeuf du Peul, on les a emmenés à la justice

klúúle $v$ (const) 1) vt rouler (la boue d'argile), enrouler (la natte, la liane) $\square$ ò pèc̀ kluu, bhéċ ò fź do kélé on roule l'argile en boules et on construit la maison (avec elles) 2) vr se rouler, se plier $\square$ mí ý yí klúúla je me suis plié 3) vi se tourner $\square$ gòn ć klúulà jásá ć zi la voiture a tourné autour de la palissade

klwà, klòà $\boldsymbol{n}$ terrain saline [naturel ou artificiel fait pour attirer des animaux sauvages]

klwáále, klòàle vi (mob) 1. maigrir, être maigre $\square$ è kwlààà il s'est amaigri 2. vieillir

klwààná <Sénoufo ?> $\boldsymbol{n}$ fétiche (fétiche importé qu'on enterre pour tuer ou chasser les sorciers)

klwáánle, klóánle v 1) vt coller 2) vi 1. s'accrocher (à - ma) yro ć klwáánnà yrì gbe do $m a$ le filet s'est accroché à une branche 2. se coller $\diamond$ yrè kwlánle ò les yeux sont collés (sommeil ou maladie) 3. être incapable de s'arrêter (par exemple, la parole) $\square$ Gùle a jà láá yan, è kwlan jan ma Gulé ne peut pas s'arrêter de parler

klwàn, klòàn $r n$ coude $\diamond$ gbe klwàn coude

klwééle, klóéle $v t$ creuser $\square$ kwè glù gbèntèn kwlee le blaireau creuse de gros trous $\diamond$ bu kwlééle creuser la tombe ; moว kwléle creuser des trous de souris (pour les chasser)

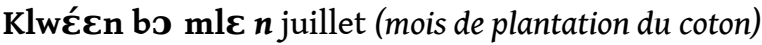

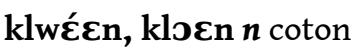

klwEn $\boldsymbol{n}$ caméléon [n'est pas mangeable]

kóbhílííi $\boldsymbol{n}$ sous-préfecture

kójó $<$ Akan> $\boldsymbol{n}$ sous-vêtement de femme

kókó n Colocasia esculenta taro (plante et tubercule) [plante cultivée pour son large tubercule, comestible et riche en amidon]

kòlay $<$ Fr. courant $>\boldsymbol{n}$ électricité, courant, ampoule $\square$ kòlay té $\varepsilon$ bhí ! allume l'ampoule !

kòmì $<$ Fr. commis $>\boldsymbol{n}$ commis (agent de l'état)

kóy $\boldsymbol{n}$ pirogue $\diamond$ yitakón pirogue

kóń $\boldsymbol{n}$ porte (en bois) $\diamond$ f́́líikón porte de la maison

kòo adj gauche $\diamond \grave{a}$ gbe kòo ta à sa gauche $\square$ gwláan mu yalàle ò ý gbe kòo ta les hommes

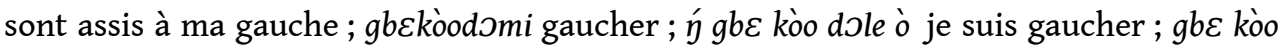
ta gaan le pied gauche

kòò $n$ 1. cuvette (en bois) 2. $r n$ portion, plat $\diamond$ báa kòo do un plat de riz

koo $n$ 1. $r n$ reins, lombes 2. rein

kóókò ke conj pour, à cause de $\square$ è nwà gè kóókò ké è jàn nrále wo kòó ni il est venu pour nous donner de bonnes nouvelles

kòókòó $n$ hémorroïde

kòònغ̀ $\boldsymbol{n}$ cuvette traditionnelle (en bois) [hors d'usage depuis longtemps]

kòrògbelèlè n Marcusenius psittacus, Marcusenius ihuysi marcusenie (poisson d'eau douce)

kósò <Dioula kósòn $>\boldsymbol{n}$ cause 
kòá (kòó+à) pron nous (incl.)... le (série contractée « sujet-objet direct »)

kòò diin $\boldsymbol{n}$ arbre (esp.)

kòó láò (kòó+láá+ò) pron nous ne... pas (1 pers. pl., incl., série contractée négative)

kJo $\boldsymbol{n}$ cage (pour transporter des poulets) $\square$ kée maa kúnlé, à kJo é líigo si tu veux attraper le poulet, il faut ouvrir sa cage. $\diamond$ maakoozi fond de la cage (par laquelle on commence à la tresser) ; volant de voiture

kòò $n$ (contr) main (paume de main) $\diamond$ à kذ̀j̀ ò flè̀ il est pauvre $\square$ à kذ̀j̀ ò éflè̀lé il n'ya rien dans les mains

Kòó ò bhe oo intrj au revoir ! (adieu à quelques personnes)

Kòó ò yi kèlé intrj à bientôt !

kòó pron nous (1 pers. pl., incl, série de base; série non-sujet ; série impérative)

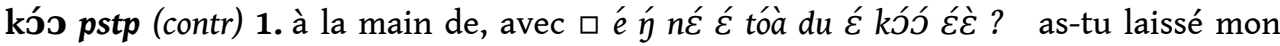
enfant avec le buffle ? 2. à cause de $\square$ Tóónc làa gbú ta gblàn kóó Toné s'est accusé luimême de peur $\square$ ýdE a ga ć kóó náá yàà so gelè Pàlii à cause de la maladie de mon père, je n'ai pas pu aller à Paris $\square$ le blaale ò pebhlele bèbè ć kóó la femme est devenue grosse à force de manger beaucoup 3. par (la valeur de l'agent dans la phrase passive) $\square$ án sćwé bhlele ò moว mu kóó mon papier est rongé par des souris $\square$ zò ć gonle ò ý ná kóó le pangolin a été vendu par ma femme 4. (marque le possesseur dans la construction possessive) $\square$ f́́ do ò ý kóó j'ai une maison

kóóle vi trembler, frétiller, se battre

kóófléy <kóó+fléy> $\boldsymbol{n}$ pauvre $\diamond$ kóófléy $m \varepsilon \varepsilon$ personne pauvre

kòòláá <kòò+láá> $\boldsymbol{n}$ (contr) paume

kòòláálííi <kò̀̀+láá+liîi> $\boldsymbol{n}$ (contr) paume (ouverte)

kòómoo, kòó mu pron nous (2 pers. pl., incl., série emphatique)

koon $n$ Bagrus bajad poisson argenté

kOOn ti $n$ Synodontis eupterus, Synodontis clarias, Synodontis ocellifer poisson-chat

kóoné $n$ colibri

kòònkoon $\boldsymbol{n}$ petit poisson (usually sold in the market)

kóówóle adj personne invulnérable au mauvais sort ou à la sorcellerie (grâce à l'utilisation des plantes médicinales magiques)

koko $a d v$ admirablement, très (intensificateur ; $\mathrm{a}-$ lre)

koloybài $\boldsymbol{n}$ chien de brousse

kolè $\boldsymbol{n}$ métier, machine-outil $\diamond$ s tan kJlè métier de tisserand (des tissus traditionnels)

kona $n$ Lemniscomys souris zèbre (à raies) [interdite à la consomation pour les clans qui ont l'antilope rayée ou la panthère comme totems]

koné $\boldsymbol{n}$ scarabée, coléoptère, bestiole

kònî $\boldsymbol{n}$ harpe-luth (instrument musical) $\square$ Soma kòní wézíi Soma joue de la harpe-luth

kòniì <Dioula> intrj c'est ça, vraiment

kònnakònna n Mycteria senegalensis jabiru (oiseau échassier à gros bec)

kòý (kòó+ý) pron nous (incl.)... me (série contractée « sujet-objet direct ») 
koygo, koyo $\boldsymbol{n}$ bosse (homme, animal)

koygod $\boldsymbol{\varepsilon}$ wlènné $\boldsymbol{n}$ chenille bossue

koygodemi $\boldsymbol{n}$ bossu

kòwlùu $<$ Dioula $>$ n Aonyx capensis loutre

kùkù $\boldsymbol{n}$ grande corbeille (pour garder des vêtements; faite de palmier) $\square$ à gbe ò kùkù tánzíi

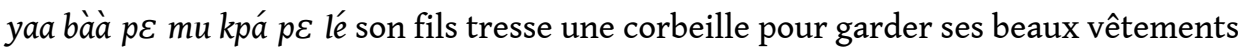

kulàle vi s'incliner, se baisser (comme en passant sous la branche d'arbre) $\square$ kula pé bhéć é kan yri gbe ć lâ abaisse-toi d'abord avant de passer sous la branche d'arbre

kúlè $\boldsymbol{n}$ tortue

kumada $<$ Fr. commandant $>\boldsymbol{n}$ sous-préfet

kùnáá $n$ famille (étendu), lignage (paternel) $\square$ YEgbe mu a kùnáá ò ple Bàànluma il y a deux familles des Yegbé à Bambaluma

kúnle $\boldsymbol{v t}$ (const) 1. attraper, saisir $\diamond$ klòsi kúnle respecter 2. remarquer $\square$ Zàle Pićli kwàn maa fàànwó yre noذ̀ Zalé a surpris Pierre en train de voler un poulet $\diamond$ gbú kúnle se comporter (faire attention au comportement) $\square$ é gbú kún kplankplan tiens-toi bien!

kuygbe $\boldsymbol{n}$ feu de brousse (type de chasse) [on met le feu à la brousse pour que les animaux se dirigent vers les chasseurs]

kùynì̀, kùylii, kúytìi <Dioula> $\boldsymbol{n}$ chef $\diamond$ swràsé mu kùgnii chef d'armée ; làkluù kùnnìi enseignant

kúlukúlu, kurukuru $n$ coléoptère (esp.)

kutuku $n$ koutoukou (boisson alcoolique forte)

kúu, kúù n souris (esp.: de brousse, mangeable, les poils un peu dressés, de couleur grise ; " petit agouti »)

kúúlu $n$ faucon

kuwî̀né $\boldsymbol{n}$ Atelerix albiventris, Erinaccus frontalis hérisson

kwa $n$ kaolin (argile blanche) [on l'applique sur le visage et le cou comme signe de joie]

kwà, kwàà $\boldsymbol{n}$ folie $\diamond$ kwàa $m a$ za acte de folie; kwàa ò à yí il est fou

kwâjàá $\boldsymbol{n}$ banane «Conakry » (Iv.)'(petite banane douce)

kwàníî, kwàní $\boldsymbol{n}$ fou $\square$ ò kwàníi bami do yà Bàànluma gblèbà ć ta on a vu un Dioula fou dans les rues de Bambalouma $\square$ ò kwàníi mu gaan wla gblún yí on met les pieds des fous dans des sabots en bois

kwe $\boldsymbol{n}$ bagage, charge $\diamond$ kwé kpále charger, faire le bagage (Afr.) $\square$ ò kwé kpa gòn $\varepsilon$ ta on met les bagages sur la voiture ; kúe kpá yre coffre à bagages

kwe $n$ Mellivora capensis ratel (animal)

kwe $\boldsymbol{n}$ Ostryoderris stuhlmannii, Xeroderris stuhlmannii arbre (esp.: aux fruits noirs et amers, dont l'huile est utilisée dans les lampes traditionnelles) $\diamond$ kwe yròn huile de " kwé "

kwéníkwé n Bucerotidae calao [mange des fruits de l'arbre ca « pommier d'aki »]

kwláan $n$ plie (poisson) 


\section{Kp - kp, comme kpáa, kpáán, kpákò}

marmite $\diamond$ lrò kpále demander ; bléć kpále envoyer un cadeau $\square$ ý náá gwlecn bléć kpàa à dò̀le ni mon frère aîné a envoyé un cadeau à sa sœur ; sćwé kpále envoyer une lettre ; yràla kpále avoir honte; són kpále mordre ; gbe kpále à kóó serrer la main de qqn $\square$ mí ý gbe kpàà à kóó je lui ai serré la main ; kpáledomaya union 2. cultiver, biner (avec la houe)

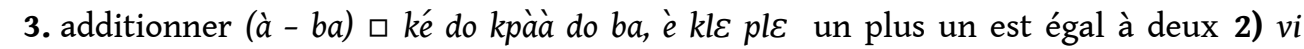
1. suivre $(q q c h-z i)$ 口 kpáá gaan glùu zi suis sa trace $\square$ ò kpàa kúu $\varepsilon$ zi bhéc̀ wà dià on a poursuivi la souris et on l'a tuée 4. attraper (maladie) $\square$ ga kpàa à ma il est tombé malade

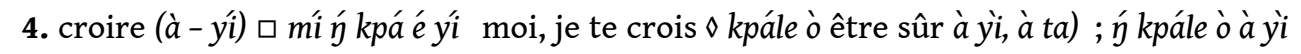
bhîi... je suis sûr que...

1013 kpálóy $\boldsymbol{n}$ fronde

1014 kpama adv 1. par force $\diamond$ kpama le yaàle mariage forcé, mariage par rapt [quand on vole la femme sans la demander en mariage auprès de ses parents; on retient la femme jusqu'à ce qu'elle tombe enceinte, puis on règle l'affaire avec ses parents et on paye la dot] 2. exprès

1015 kpàn $\boldsymbol{n}$ masque (de l'ethnie wan) 
1016 kpàn $\boldsymbol{n}$ place publique (du village) (où l'on célèbre les fêtes, et où les jeunes s'amusent)

1017 kpáykpáy adv ideo très dur

1018 kpátà $\boldsymbol{n}$ claie (table très haute composée de branches d'arbre, qui sert à faire sécher les légumes, les tissus, etc.)

1019 kpataakpa adv ideo clair, vrai $\diamond$ wlan kpataakpa vérité pure de noix ; ý kpée ma à mon avis; kpée léćdanle penser u yóo é kpée lé-danzí mèla ma éc̀ ? à quoi penses-tu? ; nile à kpéé oublier $\square$ jàn $\varepsilon$ niâ ý kpée j'ai oublié l'histoire ; kpéé léćnile être étonné $\square$ waa bhé yéle li do bhé o kpée léć-nía elles n’ont jamais vu ça, et elles en sont étonnées

1026 kpéélćníle $<$ kpéé+lé+níle> vt 1 . oublier 2. étonner

1027 kpéésíga $<$ kpéé+sít+ga> $\boldsymbol{n}$ diarrhée, dysenterie

1028 kpélikpélì $a d v$ >> ti kpélikpéli noir (précisément)

1029 kpéné $\boldsymbol{r n}$ segment (ce qui est segmenté naturellement: canne à sucre, bambou, tige de

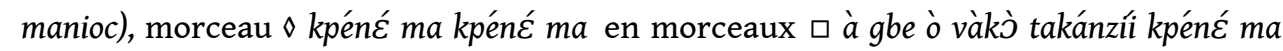
kpéné $m a$ son fils découpe des cannes à sucre en morceaux

1030 kpéy adv ideo avec les lèvres serrées, solidement $\square$ bo é líi tánle lé kpéy reste avec la bouche serrée bien fort

1031 kpengàn $\boldsymbol{n}$ arbre (esp.: perd ses feuilles, son bois n'est pas très dur, donne des petits fruits ronds non mangeables)

1032 kpeteekpe $a d v$ ideo court $\square$ zi $\varepsilon$ yòo kpeteekpe ce chemin est court

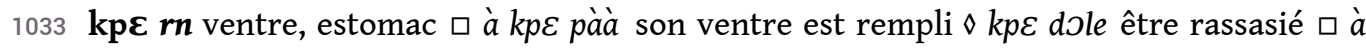
kpE laa do bhii maa il mange comme une poule (il ne peut pas se rassasier)

$1034 \mathbf{k p \varepsilon c i ̀ n \varepsilon} \boldsymbol{n}$ fourmi (petite et noire, habite dans la maison)

1035 kpè̀̀n $n$ épervier

1036 kpékpé $\boldsymbol{n}$ collant, épine, bardane (graine d'une plante sauvage qui se colle aux vêtements)

1037 kpele adj enceinte $\square$ à na kpcle ò sa femme est enceinte

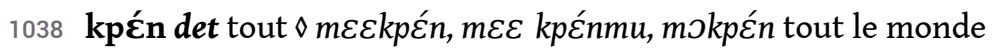

1039 kpén moo $n$ souris (de brousse, rouge)

1040 kpén $\boldsymbol{n}$ Cephalophus rufiatus céphalophe à flancs roux, biche, biche rouge

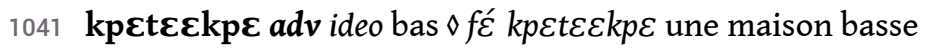

1042 kpiin adj lourd $\square$ kwé léċ kpiin ò nè $\varepsilon$ ma ce bagage est trop lourd pour l'enfant

1043 kpiinc̀ $\boldsymbol{n}$ aubergine amère

1044 kpla $n$ sorgho, gros mil (Afr.) (graminée, utilisée dans l'alimentation) 
1045

kplákásá n Paullinia pinnata liane pied de chameau, hippo cola [utilisé pour fabriquer des cure-dents]

kplankplan adj vrai, véritable, juste $\square$ jàn kplankplan vraie parole $\square$ moodomi kplankplan wlan pe le vrai guérisseur dit la vérité

kpló $\boldsymbol{n}$ anneau de bras

kplo $\boldsymbol{n}$ mille-pattes

kplélé adj odorant $\square$ làsiklò kplélé ò les parfums sentent bon

kpléykpléy n Psammophis sibilans serpent rayé de sable

kple adj 1. usé $\square$ án dunc̀ pú mu é wòo ékple lé mes chemises blanches sont usées 2. inutile, bon à rien (objets, personnes)

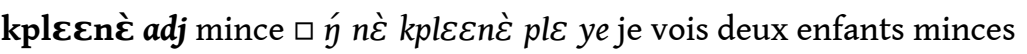

kplesetenè adj péjor. maigre, décharné

kplikplì $n$ varicelle

kplo $\boldsymbol{n}$ gourde [plante grimpante dont les fruits sont appelés courges calebasses; récipient fait de ce fruit]

kplùù onomat $\mathrm{t}$-rrr (son de voiture)

kpo $\boldsymbol{n}$ foyer (le foyer est fait de trois gros cailloux enfoncés dans le sol) $\diamond$ kpoyi te feu de foyer; kpo klele faire la cuisine $\square$ Zàlé a kpo klegbeya laa so ý ni je n'aime pas la manière dont Zalé fait la cuisine

kpokl $\varepsilon \mathrm{p} \varepsilon<\mathrm{kpo}+\mathrm{kl} \varepsilon+\mathrm{p} \varepsilon>\boldsymbol{n}$ ustensiles de cuisine (chaudières, marmites, vaisseau etc.)

kpokpàà $\boldsymbol{r n}$ association, grin [groupe de personnes qui s'entendent, qui forment des amitiés soudées et qui mangent ensemble; amis d'enfance) $\diamond$ ó kpokpàa notre association

kpolokpolo adv ideo à l'identique (par exemple, des morceaux de bois découpés)

kpomaglin $n$ Gryllotalpa africana taupe-grillon

kpònaà $\boldsymbol{n}$ igname (sorte)

kpóngán $n$ Aframomum alboviolaceum amomum (herbe) $\diamond$ kpóngán diin arbuste dont les fruits ont une pulpe comestible

kpòò $\boldsymbol{n}$ houe, daba $\diamond$ kpòo bhe la lame de la houe; kpòo yrì la manche de la houe

kpoo rn dos $\square$ le à né bo à kpoo ta la femme porte son enfant au dos

kpoti $n$ (contr) caillou

kpó det seulement, uniquement $\square$ ý bie yaga kpó là yà j’ai vu trois éléphants seulement

kpó n 1. rn genou $\diamond$ kpó yré pointe du genou ; kpó páánć rotule ;à kpó ta sur son genou ; yà kpó ziàn il s'est agenouillé 2 . cale

kpoo $n$ cicatrice $\diamond$ bhe kpoJ cicatrice ; bhle kpoJdole faire des nœuds, nouer

kpoo n plante (esp.) [plante herbacée à feuilles oblongues de 5-7 cm de long; est utilisée comme base pour les sauces]

kpò̀̀ $n$ cauri (coquillage) [coquillage ovale brillant, qui a servi de monnaie ou de decoration] $\diamond$ kpذ̀̀̀ bhe coquille de cauri

kpòkpòné $\boldsymbol{n}$ marteau

kpotookpo adv ideo petit 
kpoti $n$ silure

kpózènle vi agenouiller, être agenouillé $\square$ yòo kpózغ̀nzíi sáá $\varepsilon$ ta il est en train de s'agenouiller sur la natte

kplóò, kpro $\boldsymbol{n}$ bouillie $\diamond$ blàán kpro bouillie de banane

kploo, kproo adj mince et haut, élancé (personnes, arbre) $\square$ gwà $\varepsilon$ dwà kproo bòn dreyí l'iroko a poussé tout droit au milieu de la brousse

kplo, kpro $n$ pigeon

kpu $n$ morceau $\square$ à gbe kpu kààn on lui a coupé la main $\diamond$ lè kpu morceau coupé au bout kpúkpú adv ideo ff-ff (le bruit émis par souffler le trou du rat) $\square$ à gbe ò vli É péÉnzii kpúkpú kpúkpú son fils est en train de souffler dans le trou du rat : ff-ff

kpùn, kùỳ n 1. caillou $\diamond$ vle kpùn gbèntèn grand caillou 2. fruit (papaye, avocat; ovale, de dimension moyenne) 3. poing $\diamond \grave{a}$ gbe kpùn poing 4. objet ovale (n'importe quel objet de forme ovale et de grandeur moyenne)

\section{$L$ - I, comme la, làànć, laflé}

82 là <lè+à> pron c'est... que (marqueur de focalisation non-sujet) $\square$ ý kaa là bhlà zòn c'est du poisson que j'ai mangé aujourd'hui ; là wóle (marqueur de focalisation du prédicat) $\square$ ý kaa ć bhle là wòà zòn je l'ai mangé, le poisson, aujourd'hui (lit. c'est manger que je faisais avec le poisson aujourd'hui)

1083 lá mrph lequel (relativisateur) $\square$ mec lá sòo ć fáánlà, án yâ j’ai vu l'homme qui a volé le vélo

1084 la $n$ pluie $\diamond$ la ban il pleut ; la yrè bole la foudre jaillit $\square$ Syn. wiśi

1085 la pstp 1. sous $\square$ yri diin là sous l'arbre 2. pendant, au cours de $\diamond$ yi yaga là pendant trois jours

1086 làà $\boldsymbol{n}$ (contr) feuille (d'arbre) $\diamond$ gbelàà paume (de la main) ; gaanlàa semelle (du pied) ; Argo argent

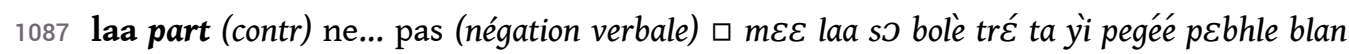
l'homme ne peut vivre sur terre sans eau ni nourriture

1088 laale $a d v$ là-bas (le plus éloigné) ১ laale bhe là-bas

1089 làànć n 1. feuille 2. médicament (herbe médicinale, comprimé, poudre, médicament liquide) $\checkmark$ lààć kpále soigner qqn (avec des médicaments)

1090 làànékpámi <làànć+kpá+mi> $\boldsymbol{n}$ docteur, médecin

1091 laaníma, laanííma, laanéma $\boldsymbol{n}$ loc haut, en haut $\square$ é gbe $\varepsilon$ wléċn laaníma lève la main en l'air $\square$ ý laaníma són la j'ai mal à une dent supérieure

1092 ladúázì $<$ Fr. ardoise $>\boldsymbol{n}$ ardoise

1093 laflé $\boldsymbol{n}$ ciel $\square$ laflé tílàá le ciel s'est assombri $\square$ Christ. Wàànbhaa ò laflé yí Dieu est au ciel

1094 làgale <là+gale> vi Euphém. être impuissant (homme) $\square$ Syn. gbà gale

1095 làglinle <là+glin+le> vt Fig. regarder au fond des choses, examiner, analyser $\square$ ké ká yaa jàn ć láglinnà, ká á yáán go épegée è jàn yóo wòa si vous observez le fond de sa parole, vous vous rendez compte qu'il a mal parlé 
1096 làkluù, làkrùù $<F r$. école $>\boldsymbol{n}$ école (institution) $\diamond$ ò làkluù yì aller à l'école ; làkluù wà école (place) ; làkluùya instruction scolaire

1097 làkluùné, làkrùn $\boldsymbol{n}$ élève (de l'école)

1098 làle vi (mob) 1. faire souffrir, faire mal $\square$ ý só ò làzí ý ma j'ai des maux de dents 2. blâmer $(q q n-t a)$ 口 Zàan làa á gbú ta Jean s'est blâmé 3. être désagréable, être difficile

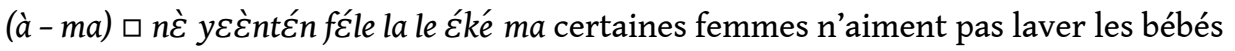

1099 làmà $n$ lampe à pétrole $\diamond$ làmà $\varepsilon$ ma wla ! baisse la lumière de la lampe

1100 lámátí $<$ Fr. amende> $\boldsymbol{n}$ amende, taxe, impôt $\diamond$ lámátí séwé facture, reçu

1101 làsìklò <Dioula lahkJlon < Fr. eau de Cologne> $\boldsymbol{n}$ parfums

1102 làsò $<$ Dioula $<$ Fr. la chaux $>\boldsymbol{n}$ ciment

1103 làwléc̀nle <là+wléc̀nle> $\boldsymbol{v}$ 1. vt ramasser $\square$ è wla É lâwléćnzíi tré ma il ramasse le couteau par terre 2. soulever $\square$ gòn $\varepsilon$ bwe é lâwlè $̇$ nlà la voiture a soulevé la poussière

1104 layrèbole $\boldsymbol{n}$ éclair

1105 le copula c'est, voilà (copule de présentation, élément de construction de la mise en relief) $\diamond$ ò gbe le ce sont leurs mains

1106 lè foc c'est... qui

1107 le $\boldsymbol{n}$ femme $\diamond$ le yalàle épouser (une femme)

1108 lé pstp avec (instr.) $\square$ ý yo po án zo lé j’abats le palmier avec ma pioche $\diamond$ núle... lé amener, apporter $\square$ mí nwà ý náá gwlen lé j'ai amené mon grand frère $\square$ à gbe yàa zan cienc̀ $\varepsilon$ lé à kóó son fils a apporté le panier

1109 le voir bhle manger

1110 lè yàà copula c'était

1111 leglízì <Fr. l'église> $\boldsymbol{n}$ église $\diamond$ leglizi fé l'église (bâtiment) $\square$ ò jà leglizì elles sont allées à l'église

1112 ledole $<\mathrm{le}+\mathrm{d}$ ole $>\boldsymbol{n}$ mariage

1113 leke $\boldsymbol{n}$ goitre $\square$ leke dwà à blinnà il a eu un goitre au cou

1114 leklezan <le+kle+zan> $\boldsymbol{n}$ célibataire $\square$ Syn. gbàà óó

1115 lenغ̀ $n$ 1. madame, femme 2. vieille

1116 Lèyonin $\boldsymbol{n}$ Leyonin (nom du masque) [un des plus dangereux masques sacrés, surtout pour les femmes]

1117 leyroy $\boldsymbol{n}$ épuisette (pour la pêche)

1118 lezip $\varepsilon<\mathrm{le}+\mathrm{zi}+\mathrm{p} \varepsilon>\boldsymbol{n}$ dot (les biens donnés selon le contrat de mariage à l'épouse) $\diamond$ lezipe klele payer la dot $\square$ wááti yekèlá ba ké wlo bhe le kle lezipe lé au temps jadis, on payait les dots avec des woro (argent traditionel) $\square$ Syn. flú

1119 lè $\boldsymbol{n} 1 . r n$ (contr) lèvre, lèvres $\diamond$ lèflé do une lèvre ; l̇̀goon menton; antenne (de l'insecte); lèmagole essuyer la bouche 2. bec (d'oiseau) $\diamond$ maagwlen lèláábà barbe du coq 3. tranchant $\square$ péćn É lé ò éyóo lé le tranchant de la hache est émoussé $\diamond$ à lè ò il est tranchant 4. partie, partie intérieure $\square$ ó bwà fé lé ma nous sommes restés à la maison

1120 lè $\boldsymbol{n}$ (contr) troupeau, troupe $\square$ zro lè gbènnè do $\varepsilon$ yaa ò sa pé ce troupeau de gazelles n'est pas petit du tout $\diamond$ lè do groupe, ensemble $\square$ dri lè do ò kánzíi laale le troupeau des vaches passe là-bas 
1121 lèbalàle $<l \grave{\varepsilon}+$ balàle $>\boldsymbol{v t}$ terminer (des paroles) $\square$ yaa yaa jàn lébalá vè̀ il ne termine pas sa parole vite

1122 lébole <lè+bole> vt nourrir, élever uǵ ká kpén yáà, bhéc̀ ý ká kpén lébwà je vous ai tous mis au monde, et je vous ai tous nourri

1123 lèbhìle $<l \grave{\varepsilon}+$ bhile $>\boldsymbol{v t}$ allumer par le bout, mettre le feu à, incendier $\square$ ò yànnì lébhi bhéć ò y $p \varepsilon \varepsilon$ kéle on allume le brandon fait de branches sèches de palmier et on brûle le cœur du palmier (pour extraire le vin de palm)

1124 lèbhúlàp $\boldsymbol{\varepsilon}<\mathrm{l} \dot{\varepsilon}+\mathrm{bhúlà+}+\mathrm{p} \varepsilon>\boldsymbol{n}$ couvercle (de marmite, casserole)

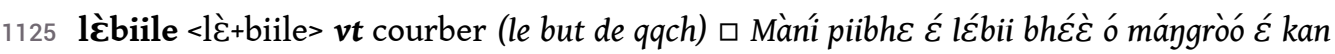
kélè Mani courbe le bout de bâton de fer pour que nous cueillions la mangue avec

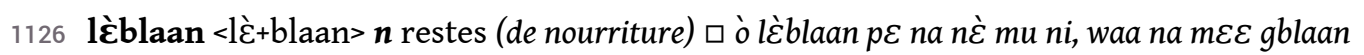
$m u$ ni on donne des restes de repas aux enfants mais pas aux adultes

1127 lèbole $<l \grave{\varepsilon}+$ bole $>\boldsymbol{v i}$ être bête, être sot (qqn immature, dont l'intelligence est attardée) $\square$ Soma lú Móólój̀ lébole ò Moloo, la fille de Soma, est bête $\square$ Syn. tabole

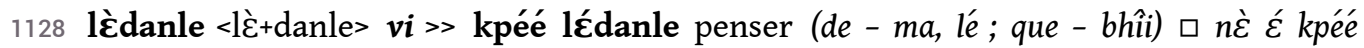

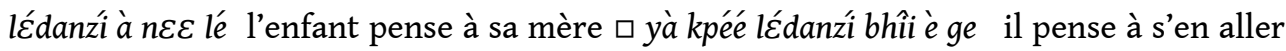
口é kpéé lédan pé bhéć é jàn wo pense avant de parler

1129 lèdoole vt 1) vt redresser (ce qui est tordu) 2) vi ajuster u é né É jóó láá lre, ké é jà kéle làkluù ýi, à lè nu doole le comportement de ton enfant n'est pas bon, si tu le fais fréquenter l'école, il va se corriger

1130 lèdole $<l \grave{\varepsilon}+$ dole $>\boldsymbol{v}$ raccommoder (remettre en bon état ce qui est coupé), rattacher (attacher de nouveau)

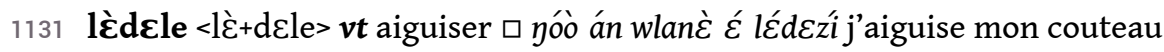

1132 lèغ̀ $\boldsymbol{n}$ (contr) devant $\diamond$ lè $\dot{\varepsilon} m i$ chef

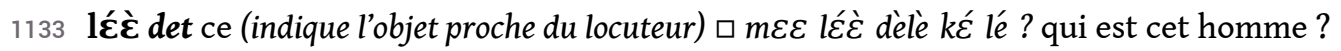

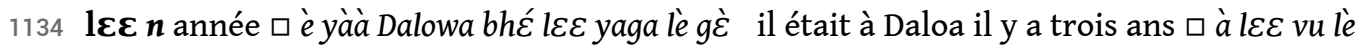

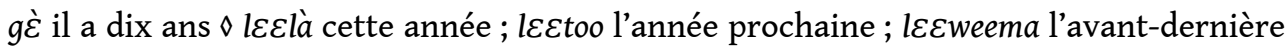

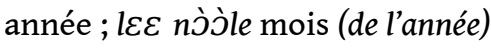

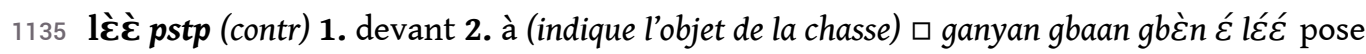
le piège à écureuils 3. de (indique la source de danger) $\square$ Táatóo wla le fé ć lâ ké yà gaanle Zàle lèc̀ quand Tato est entré dans la maison, il s'est caché de Zalé $\diamond$ gblànle à lè $\dot{\varepsilon}$ avoir peur de qqn. 4. que (indique l'étalon de comparaison) $\diamond$ dole à lè $\grave{\varepsilon}$ être plus âgé que lui

1136 lecli $\boldsymbol{n}$ année dernière, année passée $\diamond$ lecli $\varepsilon$ lé l'année dernière

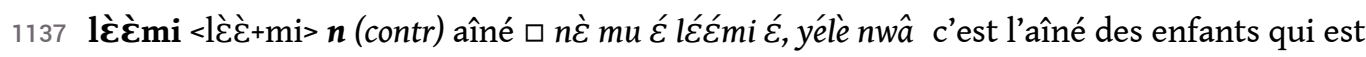
venu

1138 lecweema $<1 \varepsilon \varepsilon+w e e+m a>n$ l'avant-dernière année

1139 lèc̀ya $<l \grave{\varepsilon} \dot{\varepsilon}+y a>\boldsymbol{n}$ rôle dirigeant, autorité

1140 lègale $<1 \bar{\varepsilon}+$ gale> vi s'émousser (pour des objets en fer: couteau, machette etc.) प tré $\dot{\varepsilon}$ glò̀ya kóó, án kpòo ć légàà le sol est si dur que ma houe s'est émoussée

1141 lègbaanle <lè+baanle> $v \boldsymbol{t}$ tendre, dresser (piège) $\square$ yòo ganyan ć légbaanzí gbèn $\varepsilon$ léć il est en train de dresser un piège à l'écureuil 


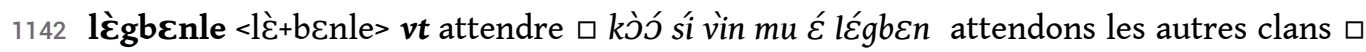
yóo ý nój̀ a súile lègbenzí j’attends que mon mari me téléphonne

1143 lèglinle <lغ̀+glinle> vt regarder si qqn arrive $\diamond$ ge à lèglin ! vas voir s'il arrive !

1144 lغ̀gole $<$ lغ̀+gole> $v \boldsymbol{t}$ enlever le son (de riz, de maïs etc.), pré-piler

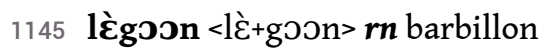

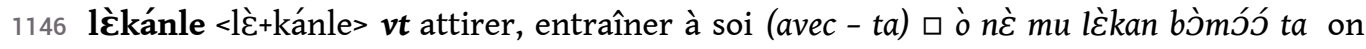
attire les enfants avec des bonbons

1147 lèklúúle <lغ̀+klúúle> vt tourner (par le bout) จ sooo léklúúle tourner le vélo $\square$ sòo ć lćklúu é $g b \varepsilon$ yic ta tourne le vélo à droite

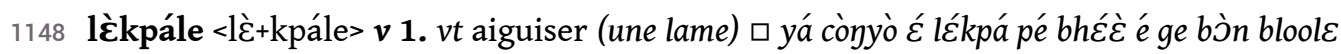
tu dois aiguiser ta machette avant d'aller défricher la brousse 2. rassembler $\diamond$ lèkpále éke ta ramasser

1149 lèkúnle <lè+kúnle> vi 1. répondre (les mots, les chansons) $\square$ náá ý náágwlecn a púbo ć lékúnle je n'ai pas répondu à la salutation de mon grand frère 2. allumer (du feu) $\square$ gázi $\varepsilon$ ́ lékún! allume le gaz ! 3. Animist. accepter l'offrande (des esprits)

1150 lèláàbà $r n$ barbe (de coq), jabot

1151 lèlâjanwomi <lغ̀+là+jan+wo+mi> n porte-parole $\diamond$ Wăànbhaa lèlâjanwomi prophète

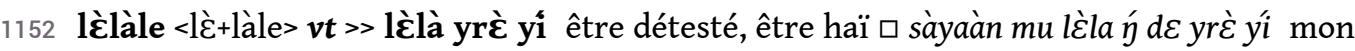
père hait les bandits

1153 lélé <Fr. l'heure> $\boldsymbol{n}$ heure $\square$ gwlaan gblan ténle ò fé $\varepsilon$ yí lélé do là le vieil homme reste adossé au mur pendant une heure $\square$ ó léćle sróa là klelè zi ć líi. nous avons huit heures de route à faire

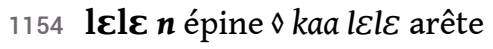

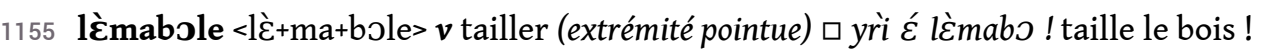

1156 lèmale <lغ̀+male> vi être trop mûr, blettir $\square$ vàngàn é lémáa bhéc̀ è klà bhòkúbhòkú, kà à na swâ mu ni la papaye est devenue trop molle, donnez-la aux cochons

1157 lèmazaabole $v \boldsymbol{t}$ contredire

$1158 \operatorname{l\varepsilon m\varepsilon } \boldsymbol{n}$ souris (esp. : très petite souris de brousse de couleur grisâtre)

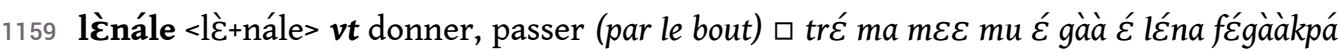
$m u$ ć ni les gens qui sont en bas donnent la paille à ceux qui la mettent sur le toit

1160 leygbe $\boldsymbol{n}$ souris (de brousse, grosse)

1161 lèpále <lغ̀+pále> vp 1. vt remplir (compléter jusqu’au bord) 2. accueillir, embrasser (un nouvel arrivé) $\square$ è nwây lépále wà É ma il est venu m'accueillir à l'entrée du village 3. vi

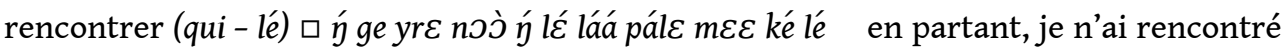
personne ১éke lèpále se rencontrer

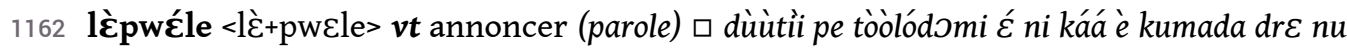
jan ć lépwe wàla mu ć ni le chef du village dit au crieur d'annoncer l'arrivée du nouveau sous-préfet aux gens du village

1163 lèsóle <lغ̀+sóle> vt ajouter, compléter, augmenter (jusqu’à une certaine mesure : un kilo, une cuvette) $\square$ yaa yaan ć lésó ajoute de l'igname !

1164 lèsónle vt allumer (feu du foyer) $\diamond$ té lésón ! allume le feu (de foyer) 
1165 lèsíle <lغ̀+síle> vi prendre de l'ampleur s'aggraver $\square$ Bùéne pegée à nòj̀ a sra ć lésia la querelle entre Bouéné et son mari a pris de l'ampleur

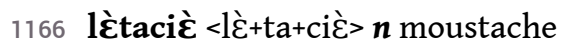

1167 létré <Fr. lettre> $\boldsymbol{n}$ lettre $\diamond$ létré dele écrire une lettre ; létré kpále envoyer une lettre

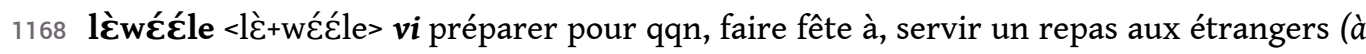
celui qui vient d'arriver) $\square$ mùà mu ba ò ćié mu lèweE bàá lé chez les Mwans, on sert du riz aux étrangers

1169 lèwìle <lغ̀+wiile> vt pré-piler (banane, igname bouillie)

1170 lغ̀wlale $<$ lغ̀+lale> $\boldsymbol{v}$ 1. vt boutonner, boucler (la ceinture) 2. faire entrer (les animaux dans leurs cages) $\square$ maa ć léwlá koj ć ýi ! fais entrer le poulet dans la cage !

1171 lèyanle <lغ̀+yanle> vi être achevé $\square$ f́é $\varepsilon$ léyáàn la maison est achevée

1172 lèyele <lغ̀+yele> vt se voir, se rencontrer $\square$ ó pegéé ý núâle laa éke lèye táý ma petite sœur et moi, nous nous voyons rarement

1173 lèzàà cíc̀ $\boldsymbol{n}$ barbe

1174 lèzàà $r n$ menton

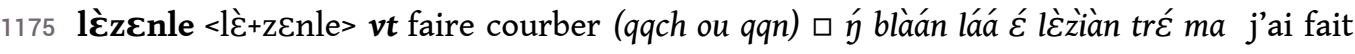
courber la feuille de banane par terre

1176 lì adv 1. pas encore $\square y ́ n \varepsilon \varepsilon$ laa yanle li gblànle lé màfá wìle lèc̀ ma mère n'a pas encore fini d'avoir peur des fusillades 2. plus (avec négation) $\square$ sj̀ ý de yàa tàmàtá mi dùn sáánníġ̀ yaa mi li auparavant mon père avait l'habitude de fumer la pipe, mais il ne fume plus 3. jamais (avec la négation) $\diamond$ li séké séké plus jamais $\diamond$ li do jamais $\square$ náa ge le lido Kòngòpla je n'ai jamais été à Kongasso

1177 liààle $\boldsymbol{v t}(\mathrm{mob})$ faire la leçon, indiquer le droit chemin, exhorter, conseiller $\square$ ké $n \dot{\varepsilon}$ ò gezí kolézi à de mu á liaa káá yà gbú kún élrele lé ye si l'enfant est admis pour le collège, ses parents lui conseillent de se comporter bien là-bas

1178 lidoò $<$ Fr. ludo $>\boldsymbol{n}$ ludo $\diamond$ lidoò zon jouer à ludo

1179 lìi $<$ Fr. lit> $\boldsymbol{n}$ lit $\square$ Syn. yilape

1180 lìi $\boldsymbol{n}$ 1. rn (contr) bouche $\diamond$ yinlìi narine; yònlíi mamelle 2. porte $\diamond$ f́́ $\varepsilon^{\prime}$ líi la porte de la maison

1181 lii $\boldsymbol{n}$ 1. $r n$ ombre $\square$ ý yáláa maygroó diin lii zi je me suis assis à l'ombre du manguier $\diamond$

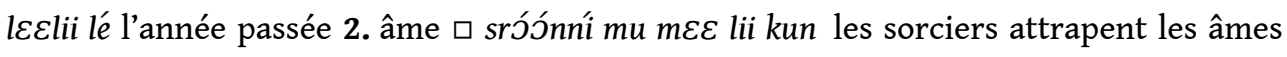

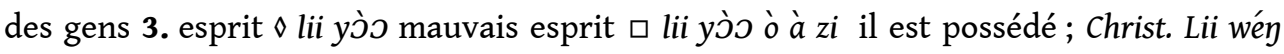
Saint Esprit 4. image, reflet $\square$ ýyóo ý gbú glìiyzí dùàlé é yí je me regarde dans le miroir

1182 lìi pstp (contr) 1. en, dans 2. sur (juste une partie de l'objet est en contact avec la surface) $\square$ yòj̀ gboo É yò zi ع́ líi une partie du tronc d'arbre est sur le chemin

1183 lìibhaale <liì+bhaale> $\boldsymbol{v}$ s'étonner, être stupéfié

1184 lìibhulale <lìi+bhulale> vt couvrir (récipient) $\square$ ý piibhe gbo ć líibhúlàá à lìitánpe ć lé j’ai couvert la marmite en fer avec un couvercle

1185 lì̀glinle $<$ liì+glinle $>\boldsymbol{v}$ provoquer, engueuler

1186 lì̀gole <lìi+gole> vt ouvrir $\square$ gòy líigole $\varepsilon$ yòo án fर́ dỉ la voiture ouverte est près de ma maison

1187 lìikáále <lìi+káále> rv bâiller $\square$ Màni é liikáálà Mani a bâillé 
1188 lìikánle <liì+kánle> vt couper la partie (d'un objet rond), pratiquer une ouverture $\square n \dot{\varepsilon} \varepsilon^{\prime}$ vàngàn ć líikáàn l'enfant a coupé le bout de la papaye

1189 lìiklwánle, lìiklóánle <lìi+kwánle> vt coller (la fermeture, y compris la plaie) $\square$ ké ò bhe tékpáa wà lìikwlaan làanć láá lé quand on soigne la plaie, on la panse avec une feuille médicinale

1190 lìikpále $v t$ pratiquer une ouverture, faire un trou débouchant (dans la gourde, faire la porte de la maison)

1191 lìile $\boldsymbol{r n}$ (contr) salive, crachat ১ lìile kláále cracher

1192 lìile $\boldsymbol{v}$ 1) $v t$ 1. effacer, annuler $\square$ wà tó $\varepsilon$ líila vóté sćwé $\varepsilon$ ta son nom a été radié de la liste électorale 2. éteindre (feu, bougie) 2) vi 1. s'éteindre 2. s'effacer

1193 Iìiplóóle <liì+plóóle> $\boldsymbol{v} 1$. vt ouvrir, détacher (qqch par le bout) 2. admirer

1194 lìisrále <lìi+srále> vt coudre pour fermer l'ouverture (ex. un sac de riz) $\square$ mlò bذ̀tò $\varepsilon$ líisráa bhćċ yá na é jiè ni couds le sac de riz et donne-le à ta grand-mère

1195 lìitánle <lìi+tánle> vt fermer

1196 lì̀tánp $\boldsymbol{\varepsilon}<$ lììtán+p $\varepsilon>\boldsymbol{n}$ couvercle

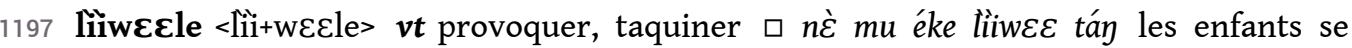
moquent les uns des autres tout le temps

1198 lìiyrééle <lìi+yrééle> $v \boldsymbol{t}$ attacher (en fermant l'ouverture) $\square$ kà kaa bòtò ć líiyrée attachez l'ouverture du sac de poisson

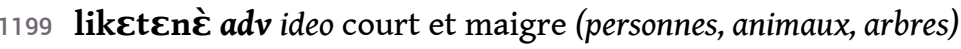

1200 lile $\boldsymbol{v}(\mathrm{mob})$ avoir pitié, gracier $(\grave{a}-m a) \square n \varepsilon \varepsilon n \varepsilon \dot{\varepsilon}$, yaa li à lù ma la mêre n'a pas pitié pour sa fille

1201 lívlù $<$ Fr. livre $>\boldsymbol{n}$ livre

1202 lòbòní $\boldsymbol{n}$ bouillie de banane (bouillie de bananes mûres melangées à de la pâte d'arachide: repas du matin habituel des Mwans au village)

1203 lòkó <Baule> $\boldsymbol{n}$ banane plantain frite à l'huile de palme aloko (Afr.)

1204 loko $n$ brume (du matin) $\square$ ké loko bwà pli kle quant la brume apparaît le matin, il y’a de la rosée

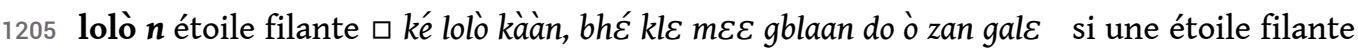
est passée, ça veut dire qu'un grand homme va mourir

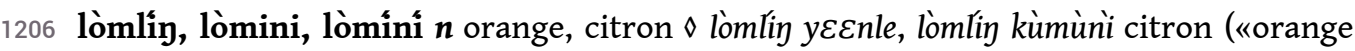
amère»)

1207 lómo n Naja nigricollis cobra cracheur

1208 lóná $\boldsymbol{n}$ taon

1209 looné $\boldsymbol{n}$ vieillarde, vieille

1210 loonغ̀, lóónغ̀ $\boldsymbol{n} 1$. Lepus sp. lièvre [personnage des contes, un trickster]

1211 lòami num le premier $\square \nabla \hat{y}$ gbe lòami lè gè c'est mon premier fils

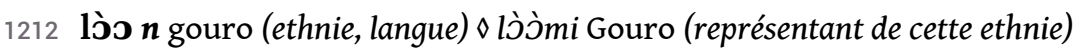

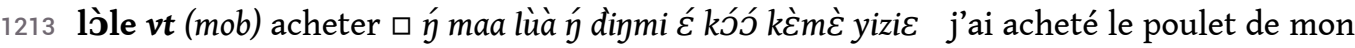
voisin à deux mille francs

1214 lòp $\boldsymbol{\varepsilon}<\mathrm{l} \grave{+}+\mathrm{p} \varepsilon>\boldsymbol{n}$ marchandise 
1215 lrèle $\boldsymbol{v}(\mathrm{mob})$ 1) vi être joli, être bon $\square$ ý lréle yiya é ni je suis plus joli que toi 2) vt faire joli, décorer, améliorer $\square$ nغ̀ mu waa f́́ lre fétí kpén lé les enfants décorent leur maison pour chaque fête

1216 lróy $\boldsymbol{n}$ mouche (grosse, qui pique)

1217 lróy $\boldsymbol{n}$ plante aquatique

1218 lrò $\boldsymbol{n}$ envie $\diamond$ à mi lrò soif $\square$ pebhle lrò ò à ma il a envie de manger; lròkpále demander (qqn - kóó)

1219 lrwale, lròàle $\boldsymbol{v}$ (mob) 1) vi 1. devenir souple, affaiblir $\diamond$ lròàle souple 2. s'apaiser $\square$ ò wi bhelé bliyni gbian ć ni bhéc̀ yaa yran ć lrwâlà on a jeté un os au chien enragé et il s'est apaisé 2) vt rendre souple, assouplir, affaiblir

\section{$\mathrm{M}-\mathrm{m}$, comme ma, maa, mabri}

ma pstp 1. à (la surface) $\square$ fotoò ć yòo fé gwle ma la photo est au mur 2. jusqu'à (sens temporel) $\diamond$ yromazi ma... jusqu'au soir $\square$ ké è san zrwanzi bhéċ è tEn yromazi ma wáá pebhlele li du matin jusqu'au soir, nous n'avons pas mangé

ma $\boldsymbol{r n}$ surface (corporelle) $\square$ ý ma É kpén kaa j’ai des démangeaisons partout

màá adv bien sûr, vraiment (commence la proposition) प màa ké yá klâ ý nu jilale á! vraiment, si tu le fais, je m'en vais

maa $n$ 1. poule, coq (générique), poulet $\diamond$ maa yren œuf (de poule); maa gbòn spaghetti ; maakJozi volant de voiture 2 . oiseau

màa $\boldsymbol{n}$ balai $\diamond$ màabhe do brindille de balai

maada $<$ maa + da $>\boldsymbol{n}$ poule

maagwlen $\boldsymbol{n} \operatorname{coq} \diamond$ maagwlen winta trò crête du coq ; maagwlen lèláàbà barbe du coq

maanغ̀ $<$ maa+nè> $\boldsymbol{n}$ oiseau

maanغ̀ gàmà $\boldsymbol{n}$ Locusta migratoria locuste

maan $\varepsilon$ d $\varepsilon$ dàan $<$ maan $\check{c}+\mathrm{d} \varepsilon+$ dàan $>\boldsymbol{n}$ lance-pierres

mabààle $<$ ma+bààle $>\boldsymbol{v t}$ choyer, chérir $\square$ yà $n \dot{\varepsilon} m u$ É mabaa ébèbè lé il chérit beaucoup ses enfants

maboole <ma+boole> $v$ 1) 1. vr se transformer (en - lé) $\square$ wlènné à mabj̀òlà tróontróongbà lé la chenille s'est transformée en papillon 2) 1. vt transformer $\square$ sróónní 
á gbe mabòj̀là soófalli lé le sorcier a transformé son fils en âne $\diamond$ jàn maboole changer d'avis $\square$ é jàn maboJ wáátí kpén ba tu changes d'avis tout le temps

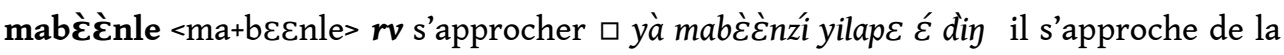
couchette

mabri $<$ Dioula $>\boldsymbol{n}$ camion

mabhole <ma+bhole> $\boldsymbol{v t} 1$. suffire $\square$ mlò bòt ̀̀ do ó mabho mle do là un sac de riz nous suffit pour un mois $\square$ Syn. yibhole 2. rincer (nettoyer à l'eau : des verres, des vêtements, des enfants) $\square$ le mu waa so mu mabho Bloo ba les femmes rincent leurs pagnes dans le Béré

1243 mabhèèle $<$ ma+bhèèle $>\boldsymbol{v} v$ s rétablir, être en voie de guérison $\square$ gadsmi $\varepsilon$ yáàle sààn à gbú ma àmasròyí à mabhèèa le malade arrive à s'asseoire par lui-même, parce qu'il est en voie de guérison

1244 mabhwèle <ma+bhwèle> $v t$ rater $\square$ ǵ bloo ś mabhwèla án di $\varepsilon$ lé j'ai raté l'agouti avec ma lance

1245 mablaale <ma+blaale> vi grossir un peu, prendre du poids a ga gwà Gboole ma, à mablààlà sááni gè Gboolé est guérie, elle a un peu grossi maintenant

1246 madole $<$ ma+dole> vt 1. reconnaître $\square$ ý drúanc̀ ć ge yre mùàn tùàbù tré ta, è nwà yièn lá lé náá madole mon neveu est allé en Europe depuis longtemps, le jour où il est arrivé, je ne l'ai pas reconnu 2. accompagner (aller avec qqn) $\square$ è jà nè $\varepsilon$ mádole wàla il a accompagné l'enfant jusqu'au village

$1247 \operatorname{mad} \boldsymbol{\varepsilon}$ sغ̀s $\grave{\varepsilon} \boldsymbol{n}$ plante (esp.)

1248 madínwóle <ma+dín+wole> vt blesser $\square$ è zro é madín wâ à gaan ma il a blessé l'antilope à la patte arrière $\diamond$ madigwóle dredre une blessure fraîche

1249 màfá $\boldsymbol{n}$ fusil $\diamond$ màfá bhe balle ; màfá wìle tirer (sur - ta)

mafáánle <ma+fáánle> $\boldsymbol{v t}$ Trad. 1 . coucher avec sa femme (avant la date reconnue par la loi des Mwans) [l'homme ne doit pas avoir de relations sexuelles avec sa femme avant que leur

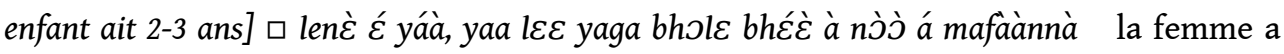
accouché d'un enfant, cela n'a pas atteint trois ans, et son mari l'a "violée avant terme » $\mathbf{2}$. violer la femme quand elle dort

1251 maféle <ma+fÉle> vt laver (les membres) $\square$ Syn. mabhole

1252 magaanle <ma+gaanle> $\boldsymbol{v}$ 1) vt $\mathbf{1}$. cacher (de lèc̀) $\square$ mí án càn yrí magaan ý né mu lèc̀ je cache ma canne d'or de mes enfants 2 . réparer $\square$ mí án gòn magaan je répare ma voiture 2) $v r$ se cacher

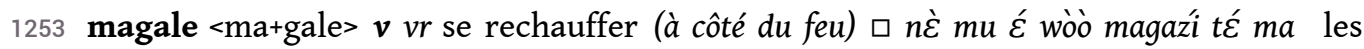
enfants sont en train de se chauffer près du feu

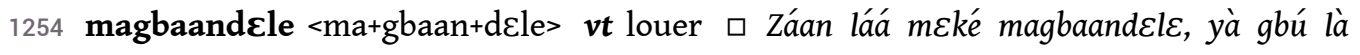
magbaandià Jean ne loue personne, il s'est loué soi-même

1255 magbaanle $<$ ma+gbaanle> $v 1$. vt retenir, retarder 2. vi se retarder $\square$ ý lú madole làkluù wà tale mí magbaannà le fait d'accompagner ma fille à l'école m'a retardé

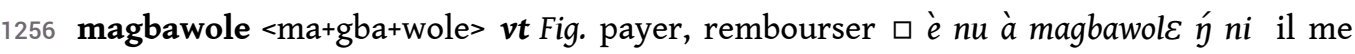
payera pour cela

1257 magbenle <ma+gbenle> vt attendre $\square$ à lù mágben sa ! attends sa fille un peu ! 
1258 magele <ma+gele> $\boldsymbol{v}$ 1. vi s'améliorer, avancer $\square$ ké è san kùnlii dre ć nu, ye ć majà depuis l'arrivée du nouveau chef, le travail est allé mieux 2. être acceptable $\square$ é wlanc̀ lá náa ý ni gè bhe, bhé mage le couteau que tu viens de me donner est acceptable maglàànle <ma+glàànle> $v \boldsymbol{t}$ masser, oindre $\square$ ò nè yíźntèn mu maglaan yròn pú lé on oint les bébés avec du beurre de karité

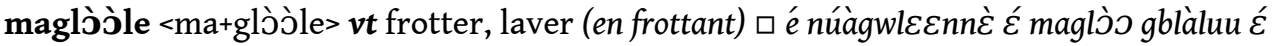
lé kplankplan lave bien ton petit frère avec l'éponge

1261 maglinle <ma+glinle> vt examiner (le corps) $\square$ dròj̀drój̀mi gademi maglin le docteur examine le malade

1262 maglòó, maygroy $\boldsymbol{n}$ mangue, manguier $\diamond$ magloó bhaa mangue (fruit); magloó bhe noyau de mangue

1263 magole $\boldsymbol{v}$ 1) $v$ t essuyer, nettoyer 2) $r v$ s'essuyer, se nettoyer

1264 majanwole <ma+jan+wole> vt parler mal $\square$ è mí là majanwozí c'est de moi qu'il parle mal

1265 makáále <ma+káále> v 1) vt gratter $\square$ mí ý lèzàa makààlà je me suis gratté le menton 2) vr se gratter $\square$ wò á makaa les singes se grattent

1266 makánle <ma+kánle> $\boldsymbol{v}$ 1) vt défricher, couper l'herbe autour de qqch $\square$ è f́ć makan il coupe les herbes autour de la maison 2) vi grandir (des enfants) [il est sous-entendu que l'enfant est déjà grand, et sa mère peut avoir des relations sexuelles avec son mari] $\square n \varepsilon \dot{\varepsilon}$ makààn l'enfant a grandi

1267 makóóle v 1. vt gratter 2. vi se sauver, s’évader $\square$ ké gwle bàláá mo kpén mú makoว quand la guerre éclate, tout le monde se sauve

1268 maklúúle <ma+klúúle> vt faire des boules (de terre, d'argile pour la construction des maisons) $\square n \dot{\varepsilon}$ mu pèc̀ makluu waa f́́ do pe lé les enfants font des boules de terre pour la construction de leur maison makpáále <ma+kpáále> vi s'apaiser, se calmer (phénomènes naturels) $\square$ Syn. mazinàle

1270 makpále <ma+kpále> $\boldsymbol{v}$ 1. vt guetter $\square$ dosòmi wi makpa bhćc̀ yà de le chasseur guette le gibier et le tue 2. vi vieillir $\square m \varepsilon \varepsilon$ makpále lè kéle c'est un homme âgé $\square$ ýde ma ò kpází mon père vieillit

1271 makúnle $v$ 1. vt choisir, trier, assortir, classer $\square$ Màní sàa dre do makwàn Mani a choisi une nouvelle natte 2. vi être choisi makwlééle $<$ ma+kwléle $>\boldsymbol{v t}$ creuser au pied (d'un arbre, d'un igname) malàle <ma+làle> vi 1. être malade 2 . faire le paresseux $\square$ ké ò Jábó winbwà yaa ge titìi à mala éyój̀lé quand on envoie Jabot, il n'y va pas du tout, il fait le paresseux

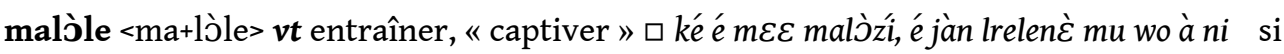
tu veux captiver quelqu'un, tu lui dis de bonnes paroles

1275 male (mob) 1. vt entendre $\square$ ý maa gwlen wèle mazí j'entends le coq qui chante 2. apprendre (facts) ם án máà kaa káá é nwâle yàláá gwlenna j'ai appris que ta petite sœur s'est mariée

1276 male vi (mob) être cuit $\square$ bàá $\varepsilon$ máà le riz est cuit

1277 málién <Fr. malien>n Malien

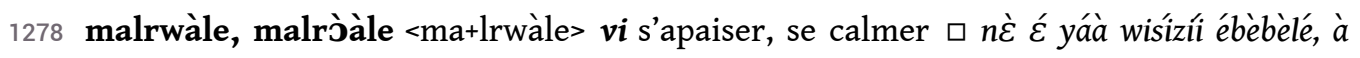
$n \varepsilon \varepsilon$ nuâ bhéc̀ yaa wisí ć malrwàlà l'enfant pleurait beaucoup, sa maman est arrivée, et ses pleurs se sont calmés 
1279 malrògole <ma+lrò+gole> $v \boldsymbol{t}$ taquiner [surtout les « cousins à plaisanterie »]

mamlààle $<$ ma+mlààle $>$ vi avoir des boutons sur le corps

mànévrù $<$ Fr. main d'oeuvre> $\boldsymbol{n}$ ouvrier

maniinàle <ma+niinàle> $r v$ se changer, changer l'aspect $\square$ wlènné à manìináá tróontróongbà lé la chenille s'est transformée en papillon

1284 manimani $a d v$ ideo >> manimani la grêle

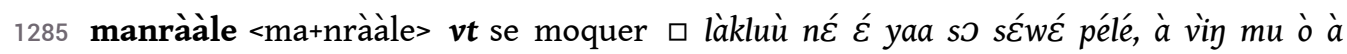
manräàzí l'élève ne sait pas lire, ses camarades se moquent de lui

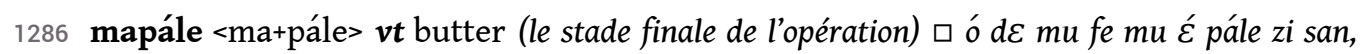
bhéċ ómoว wó fe mu $\varepsilon$ mapa nos parents commencent le buttage, et nous le terminons màsà $n$ roi

1288 masóle <ma+sóle> vi commencer à mûrir (fruits, légumes, tubercules) $\square$ yaan É maswà l'igname a commencé à mûrir

1289 masićle <ma+sićle> $v \boldsymbol{t}$ nuire (à qqn), gâter $\square$ à nùàgwlecn ý masì̀là son petit frère a gâté mon affaire

1290 masiíle $<$ ma + síille $>\boldsymbol{v t} 1$. s'adresser à quelqu'un par son nom clanique $\square$ yà masìilà Gògbe il s'est adressé à lui par son nom de famille Gogbé 2. respecter

1291 matánle <ma+tánle> $v \boldsymbol{t}$ clôturer qqch $\square$ Zذ̀ma yaa fé matààn Zoma a clôturé sa maison

1292 matón <ma+tón> adj 1. aigre, tourné, gâté (des aliments) $\square$ zièn é matón ò dozí la sauce est en train de tourner 2. Fig. mécontent, qui a l'air mécontent (de personnes)

1293 matráánle <ma+tráánle> vi commencer à mûrir

1294 matrúúy $\boldsymbol{n}$ enfance $\diamond$ matruync̀ enfant de 3-10 ans ; matrúy bhla période de l'enfance ; $y$ matrúy bhla zi dans mon enfance

1295 mavè̀̀le $<m a+v \varepsilon \grave{c} l e>v$ 1) $v t$ diminuer, raccourcir, réduire 2) vs être chétif, être maigre (homme, animal, plante) $\square$ à mavèc̀le ò il est chétif $\square$ à mavèc̀là il est affaibli $\square$ yrì diin léc mavèc̀le ò c'est un arbre chétif

1296 mawisíle <ma+wisile> vi quémander (qqch indirectement) $\square$ áli án mawisià rájó zà é yí, yaa nale ý ni j'ai même supplié pour la radio, mais il ne me l'a pas donnée

1297 mawlale $<$ ma+wlale $>\boldsymbol{v t}$ diminuer (mèche de lampe à huile)

1298 mawole $<$ ma+wole> $\boldsymbol{v t}$ crépir (la surface extérieure des murs de la maison) $\square$ kéfé dole yààn wà mawo quand on a fini de construire la maison, on la crépit

1299 màyà $n$ lutte (sportive) $\diamond$ màyà dánle lutter $\square$ Tóoné pegée Piéli màyà dan wáátí kpén ba Tone et Pierre luttent tout le temps

1300 mayààle $<$ ma+yààle $>\boldsymbol{v}$ réchauffer

1301 mayròòle $<$ ma+yròòle> $\boldsymbol{v} \mathbf{1}$. vt égratigner $\square$ Zàta a sroónè $\varepsilon$ ciémi $\varepsilon$ mayrò̀là le chat de Zata a griffé l'étranger 2. vi s'égratigner $\square$ mí bàláá, bhéè ý gbe mayròolà je suis tombé et je me suis égratigné le bras

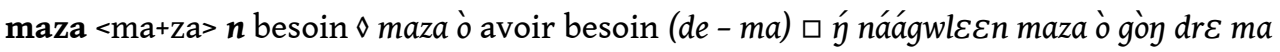
mon grand frère a besoin d'une nouvelle voiture ; zru maza désir, volonté

1303 màzí $<$ Fr. Maggie $>\boldsymbol{n}$ cube Maggi 
1304 mazinàle <ma+zinàle> vt diminuer, ralentir $\square$ yá pebhle gbsya ć mazinà il faut que tu manges d'une façon plus réservée $\square$ Syn. makpáále

mazroonle <ma+zrojnle> v 1. vt louer $\square$ è yaa gonpe mu ć mazroonzí il est en train de faire de la publicité pour ses marchandises 2. $r v$ se vanter $\square$ yà mazroonzí yaa gòn dre yí il se vante de sa nouvelle voiture

mè pron quoi ? que ? वé pe mè ? qu'est-ce que tu dis?

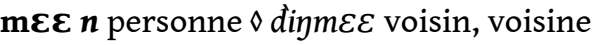

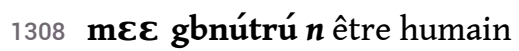

$\mathbf{m} \varepsilon \varepsilon \mid \grave{\varepsilon} \dot{\varepsilon} \mathbf{m i}<m \varepsilon \varepsilon+l \grave{\varepsilon} \dot{\varepsilon}+m i>n$ chef, dirigeant

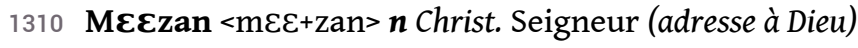

1311 mغ̀là pron quoi ? que ? (question à non-sujet) $\diamond$ mèlà ma pourquoi ? a mèlà ma é nwà Màní blaan ćć ? pourquoi est-ce que tu es venu en l'absence de Mani?

1312 méle intrj mon amie (adresse à une interlocutrice)

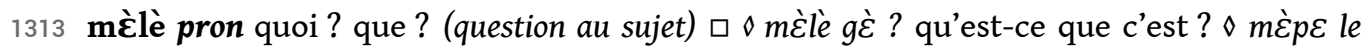
quoi ? que ? (animal) $\square m \dot{\varepsilon} p \varepsilon$ lè glù $\varepsilon$ zi ? qu'est-ce qu'il y a dans le trou ?; mèlè klà bhćغ̇... qu'est-ce qui s'est passé que...

1314 mèlì̀ <Fr. mairie> $\boldsymbol{n}$ mairie

1315 ménع, bhénè intrj mon ami (adresse à un interlocuteur homme)

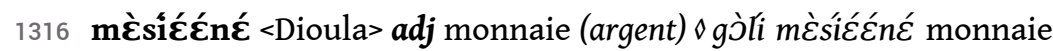

1317 métrè <Fr. mètre> $\boldsymbol{n}$ mètre

1318 mí pron 1. moi (1 pers. sg., série emphatique) 2. moi (1 pers. sg., série contrastive focalisée)

1319 mia num vingt $\diamond$ mia do vingt ; mia ple quarante ; gòli mia do cent francs

1320 mìile $\boldsymbol{v}($ mob) 1) $v t$ avaler $\square m l \varepsilon$ wi mìizi le serpent avale sa proie 2) vi être avalé (seulement au perfectif)

1321 mile $\boldsymbol{v}(\mathrm{mob})$ 1. vt boire $\square$ ò yàa yì mi páá yí autrefois on buvait l'eau de la calebasse $\diamond$ yimíyre abreuvoir 2. fumer (cigarettes) $\square \dot{\eta} n \varepsilon \varepsilon n \varepsilon \grave{\varepsilon} \varepsilon$ siklètí mi mon frère fume

1322 milízì <Fr. ministre> $\boldsymbol{n}$ ministre

1323 miyi $<$ mi+yi $>\boldsymbol{n}$ eau potable, eau buvable

1324 mizilí $\boldsymbol{n}$ mosquée

1325 mlààle $\boldsymbol{v}(\mathrm{mob}) \mathbf{1})$ vi se gonfler, s'enfler $\square$ ý plò kla bàloj̀ yí bhéc̀ bàlò̀ $\varepsilon$ mlaa je souffle dans le ballon, et il gonfle 2) vt gonfler $\square$ ý bàlò̀ mlaa je gonfle le ballon

1326 mle $n$ serpent

$1327 \mathrm{ml \varepsilon} \boldsymbol{n} \mathbf{1}$. lune 2. mois

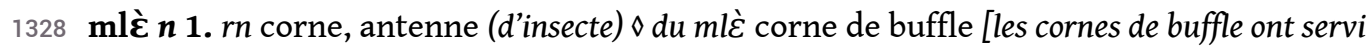
de verres pour le vin de palme] 2. flûte (instrument de musique) 3. ligne (en plantation) $\diamond$ kwléćn mlè ligne de coton

mlediin $<$ ml $\varepsilon+$ diin $>\boldsymbol{n}$ Parkia biglobosa néré (arbre avec des gousses jaunes) [arbre dont on utilise les racines et les graines en médecine traditionnelle]

$1330 \mathrm{ml} \boldsymbol{\varepsilon}$ gbli $\boldsymbol{n}$ Sida corymbosa herbe (esp.)

1331 mlekaa $<$ mle+kaa> $\boldsymbol{n}$ Gymnarchus niloticus gymnarche (grand poisson) [il fait des nids flottants et chasse les proies par localisation électrique] 

drúanc̀ ò mòzí Kànàdàa mon neveu se trouve au Canada depuis longtemps 2. avoir une longue vie $\square$ ýd$d \varepsilon$ lè wàla gblangblan lé, è mwà tré ta mon père est le plus vieux du

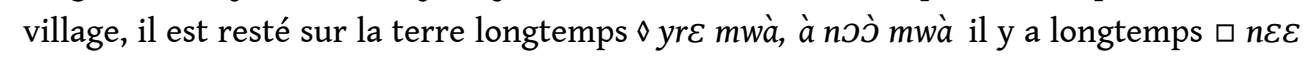

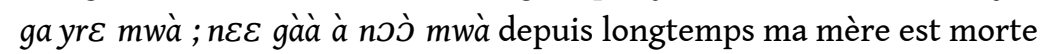

1350 mósón $\boldsymbol{n}$ boule magique (médicament traditionnel : des petites boules de couleur noir ou brun foncé) [faite d'écorce concassée, mélangée avec de la terre; est mise sur le front ou sur la plaie]

1351 mòsî $\boldsymbol{n}$ burkinabé (originaire de Burkina-Faso)

1352 mu det marque du pluriel

1353 mú pron ils (3 pers. pl., série contrastive focalisée) $\square$ mo kpén pegéé mú jóólè chacun a son comportement

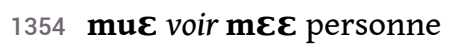

1355 muvùùy $\boldsymbol{n}$ mouche $\square$ muvùù $\varepsilon$ bwà niini yro é yí la mouche est prise dans la toile d'araignée

1356 mwà, mùà $\boldsymbol{n}$ mwan, mona (le groupe ethnique, la langue) $\diamond$ mwàmi Mwan (représentant de cette ethnie) 


\section{$\mathrm{N}$ - n, comme na, nààle, nàflò}

1358

1359 yaan mu lá yé zii bhe, nánil lè ò lè les ignames que vous voyez là sont des dons

1370 nanale voir nrale être doux

1371 náò $<\mathfrak{y}+l a ́ a ́+o ̀>$ pron je ne suis pas (1 pers. sg., série négative avec la copule)

ná part donc!

na rn (contr) épouse

náá <ý+láá> pron je ne... pas (1 pers. sg., série négative)

nàà $a d v$ au moment où, à la minute où

náa $a d v$ où?

nàà $\boldsymbol{n}$ (contr) 1. femelle (animaux) $\square$ Syn. da 2. Pejor. femme $\diamond$ nàazinغ̀ bâtard

nàágwlen, nàágloen $\boldsymbol{r n}$ (contr) grand frère, cousin (aîné) $\diamond$ náágwlen gbe neveu (fils $d u$ grand frère), fils du cousin (de la lignée du grand frère du père); nàágwlen lù nièce (fille $d u$ grand frère), fille du cousin (de la lignée du grand frère du père)

nààle $\boldsymbol{r n}$ (contr) belle-mère (par rapport à une femme)

nàflò $\boldsymbol{n}$ richesse (en argent)

nákpáa rn tante (sœur de la mère)

nále $a d v$ comment ?

nale $\boldsymbol{v t}(m o b)$ donner $(\grave{a}-n i) \square$ a ké ná $y ́$ ni donne m'en encore un $\diamond$ ná́ni cadeau $\square$ ká násáan $\boldsymbol{n}$ tante (scur cadette de la mère; fille d'un tí - un oncle maternel)

nàwé $\boldsymbol{r n}$ (contr) frère aîné (s'applique également aux fils des frères du père), grand frère (Iv.)

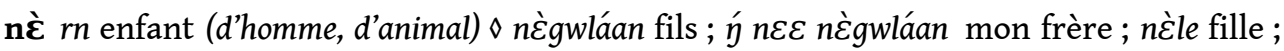

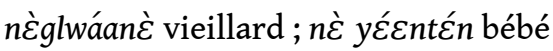

nè adj petit

nغ̀ mrph -ette suffixe diminutif

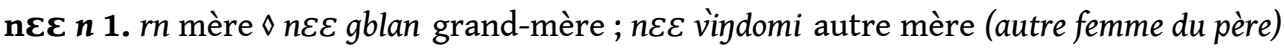

2. parente aînée, épouse du frère aîné

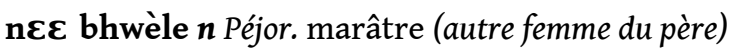

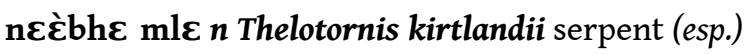

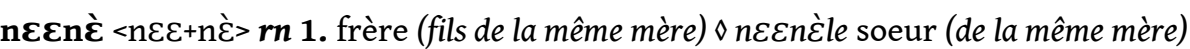

n $\varepsilon \varepsilon n \varepsilon ̀ y a<n \varepsilon \varepsilon+n \varepsilon ̀+y a>n$ parenté, liens du sang, lien entre la mère et l'enfant

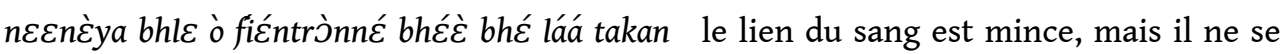
rompt pas

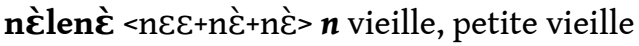

nèyákle $<$ nc̀+yá+kle> adj stérile (humains ou animaux)

nèyále $<$ nغ̀+yá+le> $n$ sage-femme

ni pstp à (valeur adversative)

nì̀ $\boldsymbol{r n}$ (contr) vie $\square$ mle nìi ò à wee yí la vie du serpent est dans sa queue $\diamond$ niima en pleine mesure, de tout coeur ; à nii ziàn ta il a soupiré ; é niigole zà do yì dédier sa vie à qqch 
1387 niinàle $\boldsymbol{v}(m o b)$ 1) vi 1. se retourner (en gardant la position couchée) 2. se retourner $\square$ ó níinàá ó zàn nous nous sommes retournés $\square$ náá níinàle li gban do wáa wà ć ta je ne suis jamais retourné à notre village 3. changer d'avis, cesser (de faire qqch) $\square$ Màní nì̀inàzíi yaan wloo yre noj̀ Mani n'a pas creusé d'igname (et se retourne à village) 2) vt 1. tourner $\square$ à niinà à gbe ta tourne-le sur l'autre côté 2. rendre $\square$ ý dunc̀ ć níinàá Màní ma j'ai rendu la chemise à Mani 3. mettre à l'envers (un vêtement) $\square$ dunc̀ ć niinàle ò le vêtement est mis à l'envers

1388 niini $\boldsymbol{n}$ araignée $\diamond$ niini yro toile d'araignée

1389 nìiníí $\boldsymbol{n}$ froid $\square m \varepsilon \varepsilon$ bèbè gàà nìiníi ć kóó beaucoup de gens ont péri par ce froid $\diamond$ nìiníi ò ; niìníi klele il fait froid ; nìiníi demi qui a la fièvre

1390 nile $\boldsymbol{v}(\mathrm{mob}) \mathbf{1})$ vi se perdre $\square$ nغ่ $\varepsilon$ nía l'enfant s'est perdu $\diamond$ nile à kpée être oublié $\square$ gòli ć zà nî̀ ý kpéé wàla j'ai oublié l'argent au village 2) vt perdre, faire disparaître $\square$ sróónní mu meE nu lii ni les sorciers prennent les âmes des gens

1391 nòó $\boldsymbol{r n}$ trompe (d'éléphant)

1392 nó $\boldsymbol{n}$ barrage (sur la rivière, pour la pêche)

1393 noj̀ $\boldsymbol{n}$ (contr) 1. endroit (n'est pas utilisé seul) $\diamond$ tóle à noj̀ abandonner; ò à noj̀ être présent 2. où $\square$ náá nój̀ do je ne sais pas où (réponse à une question)

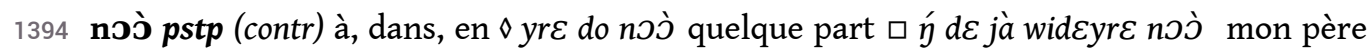
est parti à la chasse

1395 nò̀̀ $r n$ (contr) mari

1396 nòòle $\boldsymbol{v}(\mathrm{mob})$ 1) $v t$ compter $\square$ gbakpami yaa yaan bhle noว les cultivateurs comptent les

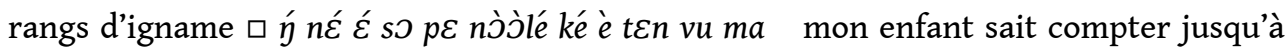
dix $\diamond m l \varepsilon$ nòj̀le mois (de l'année) 2) vi être compté $\square n \varepsilon \dot{\varepsilon} m u$ ć nóóla les enfants ont été comptés

nrààle $v(m o b)$ 1) vi être doux, être bon, devenir doux, devenir bon (à qui - ma) $\square$ wi nralà la viande est devenue bonne au goût $\square$ nràle éké ma laa ò wà É ta ils sont en mauvais termes dans ce village $\square$ Infant. bhlàa zru nana le foie du mouton est bon $\diamond$ jàn manrààle se taquiner, se moquer mutuellement (relations de cousinage de plaisanterie) $\square$ jàn ma nra ò lò̀̀ mu pegée yàòbá mu plè̀ il y a plaisanterie entre les Gouro et les Yacouba ; liii nráále dire des mots doux, chanter bien; Christ. Zèzù a jàn nráále La bonne nouvelle de Jésus 2) vt faire doux, faire bon $\square$ màzí zièn nraa le cube « Maggi » rend la sauce bonne

1398 nraleya $n$ douceur

1399 nranغ̀ $\boldsymbol{n}$ (contr) petit enfant, descendant

1400 nre $n$ langue (Anat.)

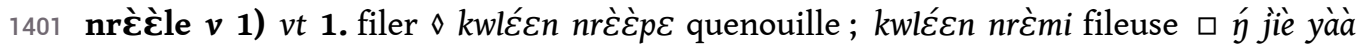
kwlécn nrèc̀mi gblan lé ma grand-mère était une grande fileuse de coton 2 . conduire (voiture) 2) vi être filé $\square$ kwlécn ć nrèlà ce coton est filé

1402 nri $n$ 1. trace, empreinte $\diamond$ gbe nri empreinte de doigts 2. ligne (trace d'écriture), plan $\square \hat{y}$ $f_{\varepsilon} \varepsilon^{\varepsilon}$ nri kan je trace le plan de la maison

1403 nró <Dioula> n lait $\diamond$ nró tónle lait aigre ; nró yí lait (y compris le lait condensé) ; nró pii lait en poudre

1404 nu $a d v$ ici (avec négation) $\square$ Olivier láò nu Olivier n'est pas là 
1405 nu mrph marqueur de futur

1406 nule vi (mob) venir $\square$ ý lú nu dimàsí ma ma fille vient le dimanche $\diamond$ nule à lé apporter, amener $\square$ ý nwâ án dunc̀ ć lé Bajo ni j'ai apporté mes habits à Badjo

1407 nwâ $\boldsymbol{r n}$ frère cadet, sœur cadette

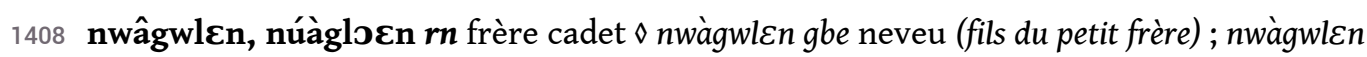
lù nièce (fille du petit frère)

1409 nwâle $r n$ sœur cadette

\section{$N-\eta$, comme ńgbhlo, ńgbhlokpàpe, ńkènà}

1410 ý pron 1. je (1 pers. sg., série sujet de base) 2. me (1 pers. sg., série non-sujet)

1411 ýciè <Dioula> intrj merci $\diamond$ ká ýcie merci (à de nombreuses personnes)

1412 yéé $<y ́+e ́>$ pron je te (série contractée "sujet-objet direct »)

1413 ýgbhlo, ýgblò $\boldsymbol{n}$ 1. $r n$ tête 2. régime (des fruits) $\diamond$ se ýgbhlo régime de graines de palme

1414 ýgbhlokpàp $\varepsilon<$ ýgbhlo+kpà+pe> $\boldsymbol{n}$ appui-tête (traditionel, en bois)

1415 ýkò intrj bienvenue, bonne arrivée, salut! (à qqn qui est venu du marigot ou du puits) $\diamond$ káýkj̀ salut (la même salutation adressée à plusieurs personnes)

1416 ýkغ̀nà intrj bon matin ! bonjour! (la salutation du matin adressée à une personne) $\square$ ýkغ̀nà oo ýkغ̀nà, Po bonjour, Po !

1417 ýkoygo intrj bienvenue, bonne arrivée, salut ! (à personne venue des champs)

1418 ýỳ intrj oui

1419 yóò, ýyóò <ý+ò> pron je suis (1 pers. sg., série contractée avec la copule)

1420 ýsغ̀ intrj mes condoléances $\diamond$ ká ýsc̀ ga ma acceptez mes condoléances pour

1421 ýsغ̀غ̀ye (Dioula) intrj soyez le bienvenu, bonne arrivée, salut! (à une personne qui est venue ou revenue) ýtèlè <Dioula> intrj bonjour ! (salutation de midi) ýténd $\varepsilon$ intrj bonjour (réponse à une salutation - un homme répond à un homme) ýtÉnnè intrj bonjour (réponse à une salutation - un homme répond à une femme)

\section{0 - o, comme ò, ómวد, oo}

ò copula être $\diamond$ ò à zi (bhîi) vouloir $\square$ ýò à zi bhîi ǵ ge je veux partir ; ò zan arriver $\square \grave{a}$ zrù ò zan sa tante arrive

ò pron 1. ils (3 pers.pl., série sujet de base) 2. les, leur, eux (3 pers.pl., série non-sujet)

1431 óó conj n'importe... $\square$ ké $m \varepsilon \varepsilon$ óó $m \varepsilon \varepsilon$ lá núa kà yì ná à ni peu importe qui est venu, donne-lui de l'eau ! 


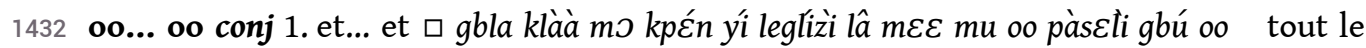
monde avait eu peur, les fidèles et le pasteur lui-même 2. (avec NEG) ni... ni $\square$ Màní oo, Zàle oo, ò do kpó láá ò làkruù wàla ni Mani, ni Zele ne vont à l'école

1433 oo part 1. est-ce que (marque de question générale avec doute; souvent avec dòké) $\square$ dj̀ké yaa $p \varepsilon$ ć yélè oo? peut être, c'est sa chose ? 2. expression du doute, incertitude, surprise $\square$ dذ̀ké è $p \varepsilon$ bhlele oo peut-être, il veut manger $\square n \varepsilon \grave{\varepsilon}$ ć bòmóó lúa oo figurez-vous, l'enfant a acheté des bonbons 3. marque de préventif $\square$ yáá é ténle fé $\varepsilon$ yi oo prends garde de ne pas t'adosser au mur 4. malgré $\square$ áliké à gaan ò làzí oo, è blasan a ta malgré le fait que son pied lui fait mal, il court

1434 óó part donc

\section{כ - כ, comme J́nวn}

\section{ónon intrj non}

\section{$\mathrm{P}-\mathrm{p}$, comme páá, paan, pàsclì}

páá $n$ calebasse [récipient formé du gros fruit du calebassier ou de différentes espèces de cucurbitacées, vidés et séchés]

páále vt (mob) piquer (d'insecte) $\square$ ý kúnle yri ma ké gbèesáàn ý páàlé quand j'ai saisi le bois, une guêpe m'a piqué

1438 paan $\boldsymbol{n}$ arbre (esp.: gros tronc et grandes feuilles)

1439 pààn $\boldsymbol{n}$ hie, dame (instrument de damage le plancher)

1440 pàcáyà $\boldsymbol{n}$ Heterotis niloticus heterotis, poisson-chien [poisson à corps long qui peut respirer l'air atmosphérique]

1441 pále $\boldsymbol{v}$ 1) vt 1. remplir $\square$ ò waa kóy É páa kaa ć lé ils ont rempli leur pirogue avec du poisson 2. butter (igname) $\diamond$ fe pále former des buttes 2) vi se remplir

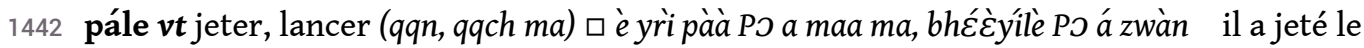
morceau de bois au poulet de Po, c'est pourquoi Po l'a frappé $\diamond$ lèpále rencontrer

1443 pánpán, pápán adv ideo très tranchant $\square$ Táatóo a còyyo ć lé ò pánpán gbú la machette de Tato est vraiment tranchante

1444 páypáy adv ideo vivement (de lumière) $\square$ yrèté bhíle páypáy ć la $m \varepsilon \varepsilon$ yrè ba la lumière vive du soleil fait mal aux yeux

1445 pápá ideoph très (intensificateur à té) पà té ò pápá c'est très chaud

1446 pàselì $<$ Fr. pasteur $>\boldsymbol{n}$ pasteur $\square$ Syn. bhlàatagbinmi

1447 pàsiàngà $\boldsymbol{n}$ danse (d'origine sud-américaine; de réjouissance, accompagnée par les tambours)

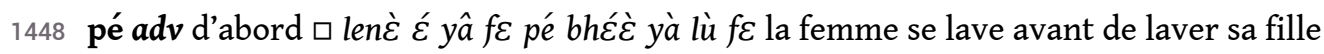

1449 péé $n$ morceau $\diamond$ pée dele déchirer $\square$ mí án dunÈ ć péćdia j’ai déchiré ma chemise (exprès)

1450 pegeé conj (contr) et, avec $\square$ yrì ع́ kpén pégeé bhé bhaa lè chaque arbre a son fruit

1451 pele $v(m o b)$ 1. vt dire $\square$ Màní á pía káá yà lòlé Mani a dit qu'il voulait l'acheter $\diamond$ zà pe demander qqn en mariage $\square \dot{y} d \varepsilon$ mu le plemi do zà pèà ý ni mes parents m'ont proposé une deuxième femme 2. ordonner (avec négation), interdire 3. avoir l'intention (de faire 
qqch) $\square$ Màní yàà pe yà lòlé Mani avait l'intention de l'acheter 4. il dit (la formule de quotation)

1452 pénî $\boldsymbol{n}$ voir pléy aiguillon

1453 péypéy adv intens $\diamond$ pú péppéy blanc

pépé $\boldsymbol{n}$ baluchon (petit paquet de vêtements)

péù adv ideo vite (à toute vitesse)

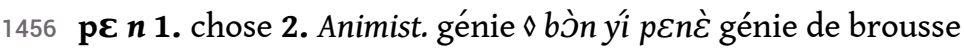

1457 p $\varepsilon$ pron 1. quelque chose $\square$ è $m \grave{\varepsilon}$ p là bhle? qu'est-ce qu'il mange? 2. quelqu'un 3. celui-ci $\square$ mlèsiennú do ná ý ni, án so é yísráa $p \varepsilon$ lé donne-moi une aiguille pour rapiécer mon pagne 4. quelque chose (substitut d'objet direct avec certains verbes bivalents) $\checkmark$ Zàan $p \varepsilon$ fàànnà pitikí do là Jean a cambriolé une boutique

1458 pebhle $<$ p $\varepsilon+b h l e>n$ nourriture

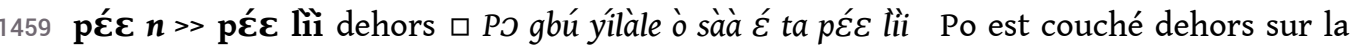
natte

1460 pè̀̀ $\boldsymbol{n}$ (coord) 1. argile 2. banco (Afr.) $\diamond p \grave{\varepsilon} \dot{\varepsilon}$ f́́ maison en terre battue 3. pâte $\diamond$ farini pćc̀ pâte de farine de blé

1461 péćle $\boldsymbol{v}(\mathrm{mob})$ 1) vi 1. éclater, exploser $\square$ bòm péćlà la bombe a éclaté $\square$ Zذ̀ma yaa bàlò̀

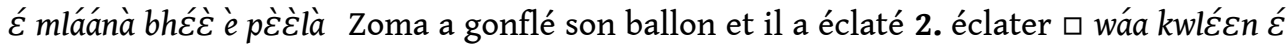
péćlà, wó ò gezí à gole notre coton a éclaté, nous allons le récolter 3. sonner, éclater $\square \grave{\eta}$ gblaan gblà péćle é léć j'ai peur du grondement du tonnerre 2) vt crever, rompre $\square n \grave{\varepsilon}$ ć bàlò̀ pèc̀là l'enfant a crevé le ballon

1462 péćn $\boldsymbol{n}$ petite houe (à manche court)

1463 péćnle $v$ 1) $v t$ 1. attiser (le feu) 2. nettoyer par soufflage, enlever qqch en soufflant 3. enfumer (façon de la chasse) ৩ plo péćnle souffler $\square$ nè ć plo péćnlà bàá $\varepsilon$ ma l'enfant a soufflé sur le riz 4. purger qqn avec un clystère 2) vi être soufflé, être ravivé ( $(u f e u) \square$ té $\varepsilon$ é $\varepsilon^{\varepsilon}$ nzii on ravive le feu en soufflant

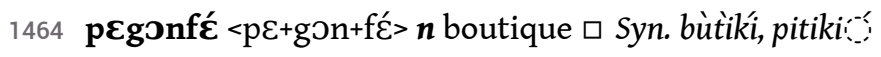

1465 peké pron rien, quelque-chose

1466 p $\boldsymbol{\varepsilon}$ lî̀p $\boldsymbol{\varepsilon}<\mathrm{p} \boldsymbol{\varepsilon}+$ liì $+\mathrm{p} \boldsymbol{\varepsilon}>\boldsymbol{n}$ couvercle

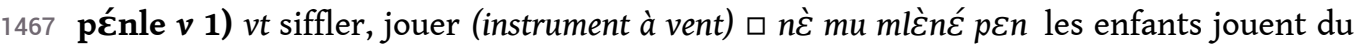
cor $\diamond$ srò pénle siffloter ; mlè pénle jouer de flûte 2) vi siffler (le sifflet), émettre un son, jouer (d'un instrument à vent)

1468 pè̀

1469 pérìmi $<$ Fr. père+mi $>\boldsymbol{n}$ catholique

1470 pérìya $<$ Fr. père+ya $>\boldsymbol{n}$ catholicisme

1471 pićsì $<$ Fr. pièce $>\boldsymbol{n}$ pièce, papiers

1472 pìgìnì $\boldsymbol{n}$ pigini (argent traditionel)

1473 pii $\boldsymbol{n}$ (contr) 1. poudre $\diamond$ màfá pii poudre de fusil 2. pâte $\diamond$ blèc̀ pii pâte d'arachide

1474 pii $r n$ vomissement $\diamond$ pii dozí ý zru ta j'ai la nausée

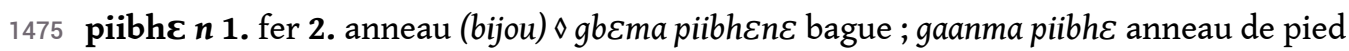

1476 piibh $\varepsilon$ gbo $<$ piibh $\varepsilon+$ gbo $>\boldsymbol{n}$ marmite en fer 


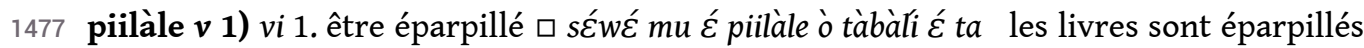
sur la table 2. s'en aller, se disperser (assemblée, assistance) $\square$ ké bhee é píiláá, mo kpén jila quand le public des funérailles se disperse, tout le monde rentre chez soi 2) $v t$ éparpiller

1478 pîile v 1) $r v$ vomir $\square$ ké ý wè̀̀n miì mí ý pi si je bois du vin, je vomis 2) vt provoquer des vomissements $\square$ wè̀̀n $m \varepsilon \varepsilon$ É píizíi le vin a fait vomir cet homme

1479 píile $\boldsymbol{v t}$ forger $\square$ ýò gannan píizii je suis en train de forger un piège

1480 pílá-pílá adv ideo vite (en trottinant)

1481 pinàle v 1) vi 1. sauter $\square$ pinà mángròo $\varepsilon$ zi bhéċ yá kan saute vers la mangue pour la cueillir $\diamond$ pinàle gaan do ta sauter sur un pied 2. sauter par-dessus $\square$ pinà yrì gboo $\varepsilon$ ta saute par-dessus le tronc 2) $v t$ faire sauter, laisser sauter $\square$ ý $n \grave{\varepsilon}$ É pinnáa yrì ta j'ai fait sauter l'enfant par-dessus un arbre $\diamond$ Euphém. lè ̇̀ pinàle avoir l'érection $\square$ ké bhoo lè̀े

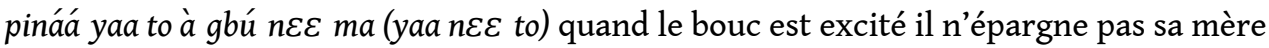

1482 pipii onomat bip-bip (son de klaxon)

1483 pitikí $<F r$. boutique> $\boldsymbol{n}$ magasin, boutique $\square$ Syn. bùtikí, pegonfé

1484 -pla mrph 1. village (dans des mots composés) 2. village (suffixe des noms des villages) $\triangleright$ yipla en sommeil $\square$ nc̀ mu ò yipla les enfants dorment

pláále vt 1. laper, lécher $\square$ zo do ò drò pli é pláázíi un margouillat lape la mousse de vin

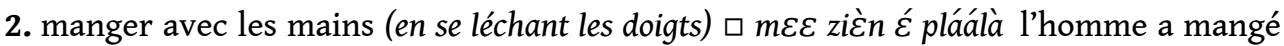
toute la sauce

plángle vt 1. attirer (une personne avec des mots) $\square$ preidaàn ò meE mu É pláánzíi le président est en train de attirer les gens 2 . draguer (une femme), séduire $\square$ án pláánlà yi do kpó ć lé j’ai dragué cette femme le même jour

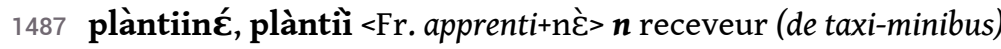

1488 pleka n Cephalophus jentinki céphalophe

1489 pley pstp 1. parmi, entre, chez $\square$ é né mu síi ó plè̀ appelle tes enfants chez nous 2. jusqu'à $\diamond$ yromazi bhole plè̀ jusqu'au soir

1490 pléy, péni $n$ 1. dard, aiguillon $\diamond$ zròbhé pléy l'aiguillon de l'abeille; gààplén, gààpéni chiendent (herbe piquante) 2. tuyau (pour recolter le jus du palmier)

1491 plè̀go <plèn+go> $v$ s'écarter

1492 pléypléy $a d v$ entièrement, totalement

1493 plé $n$ marché

ple num deux $\square$ do ké (bhéṡ) do bhé kle ple; do bole do ba bhé kle ple un plus un font deux $\diamond$ pleple deux fois $\square$ bhé láá kle pleple cela ne se répète pas

1495 plé rn prix, valeur $\square$ wòo dri ć do plé pézíi zrù ni, ò pégeé Sre lé la tante et Sre discutent le prix d'une des vaches $\diamond$ à plé láá ò ce n'est pas cher; pléle cher $\square$ ò gòy pléle mu gon Bíjàn on vend des voitures chères à Abidjan; plé péle marchander

1496 pléclé $a d v$ tôt $\square$ ý $n w a ̂$ éplé $\varepsilon$ lé je suis venu tôt

1497 plégonmi $<$ ple+gon+mi> $\boldsymbol{n}$ marchand $\square$ Mùà $m u$ ò sáánígè bhîi plégJnmu lé il y a très peu des Muan qui sont commerçants

1498 plégonyr $\varepsilon<$ pl $\varepsilon+g o n+y r \varepsilon>n$ place du marché

plemi adj deuxième 
1500 plí $n$ rosée $\square$ ké bie kààn yre lá nój̀, pli láá bo bhé nój̀ là où l'éléphant passe, il ne reste plus de rosée

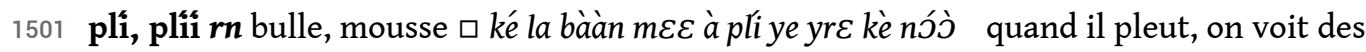
bulles d'eau par ci par là $\diamond \grave{a}$ plíi do fais-le mousser !

1502 plííle vi (mob) 1. avoir des bulles, bouillonner, écumer, faire des bulles (des enfants) $\square$ saflée plii le savon mousse 2. bouillir $\square$ se É plíizíi les graines de palme bouillent 3. être nerveux $\square$ à zru plíile yàà il était nerveux

1503 plímù <Fr. plume>. $n$ plume (pour écrire)

1504 plin $\boldsymbol{n}$ tambour $\diamond$ plin dele battre le tambour

1505 plò n 1. vent 2. air

1506 ploglámù <Fr. programme> $\boldsymbol{n}$ programme

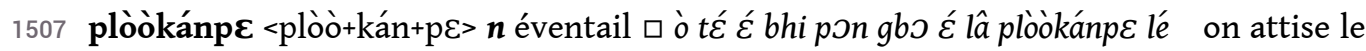
feu sous le canari de foutou avec un éventail

1508 plóóle $\boldsymbol{v}$ 1) $v t$ 1. détacher $\square m \varepsilon \varepsilon$ É yòo gàà dri ć plóozíi l'homme détache les piles de paille 2. laisser, libérer 2) vi se détacher

1509 plò $\boldsymbol{n}$ Saba senegalensis liane saba (plante grimpante)

1510 plotó >> plotó ye $\boldsymbol{n}$ travail forcé (au temps de la colonisation) $\square$ Syn. sitikúy

1511 poli $\boldsymbol{n}$ crapaud

1512 pòlisièmi $<$ Fr. policier + mi $>\boldsymbol{n}$ policier

1513 polu $n$ ampoule, cloque $\square$ polu dwà ý gaan ma j'ai une ampoule au pied

1514 poo $n$ hache

1515 pòòmi $\boldsymbol{n}$ un tel (remplace le prénom; est utilisé aussi comme salutation quand le prénom de la personne est inconnu ou oublié) $\square$ bhéċ é pe oo ! pòmi et tu dis : « Oh ! telle personne !»

1516 póó $\boldsymbol{n}$ vague $\diamond$ yi póó vague

1517 poonغ̀ $\boldsymbol{n}$ aubergine

1518 póónle $\boldsymbol{v}$ se libérer, se dispenser (de travail) $\square$ ké ká yáàn yewole lé bhéc̀ ká póón quand vous finissez le travail, vous êtes libres $\diamond$ à póónle ò il est libre, il n'est pas occupé ; à póónle laa ò il est préoccupé, il est triste $\square$ é wleyí póónle laa ò ton visage est triste

1519 póle $v$ (mob) 1) $v t$ abattre, faire tomber (arbre) $\diamond$ yripómi bûcheron; bi

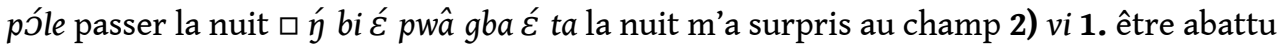

1520 póli $<$ Fr. port> $\boldsymbol{n}$ port

1521 pon $\boldsymbol{n}$ foutou [boulette de banane et manioc ou de manioc seul; est servie avec différentes sauces] $\square$ mwà ta waa súglùu pon na cié mu ni en pays mwan, on ne sert pas de foutou de manioc à un étranger pónbhé <Fr. pompe>n 1. pompe, seringue ১pónbhé ýi engrais (liquide)

pónle $v$ 1) vt 1. percer $\diamond$ trón pónle percer les oreilles; zi pónle tracer une voie, faire un chemin 2. piquer 2) vi (seulment Prf) être percé

$$
\text { pónní } n \text { pointe, clou }
$$

pòy $<$ Fr. pont $>\boldsymbol{n}$ pont

pósì $<$ Fr. poste $>\boldsymbol{n}$ radio, poste de radio

prótóye $n$ voir prótó travail forcé 
1528 prèc̀loy voir klè̀ेloy trompette

1529 preidaàn $<$ Fr. président $>\boldsymbol{n}$ président $\square$ Syn. meEwinmi

1530 pu $<$ Fr. pou $>$ n pou

1531 pú adj blanc, clair (couvre les couleurs très claires) [c'est un symbole de pureté et de bien : les gens enduisent les visages avec la cendre blanche en signe de joie; c'est aussi la couleur des forces magiques et supernaturels; celui qui va effectuer des danses rituelles tache son visage avec de l'argile blanche; les esprits de la brousse aussi sont blancs]

1532 púbole vt saluer $\square$ Kiló nú lá wóa ké è gelè ý de púbole Kilo est venu et est allé saluer mon père

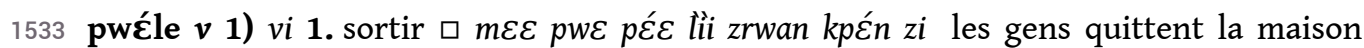

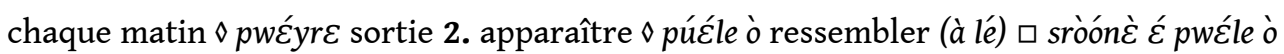
trwàné $\varepsilon$ lé le chat ressemble à la panthère 2) vt 1. sortir $\square p \varepsilon$ óó $p \varepsilon$ lá wòo gè, kà ò kpén púc péclì faites sortir toute chose qui se trouve ici

\section{$R-r$, comme ràábu, rájo, rúsi}

1534 ràábu, ràábí $n$ arabe

1535 rájó $<$ Fr. radio $>\boldsymbol{n}$ radio

1536 rúsì $<$ Fr. russe $>\boldsymbol{n}$ russe

\section{S - s, comme sá, saa, saad $\varepsilon$}

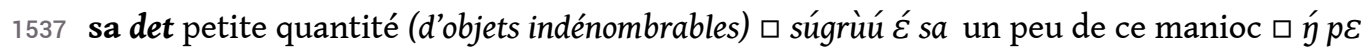
sa bhlà j'ai mangé un peu

1538 sá $\boldsymbol{n}$ arc $\diamond$ sá bhe flèche $\square$ è yaa sá pégeé à bhe mu ć sizíi il prend son arc et ses flèches

1539 sáa n 1. Alestes marcolepitodus aleste (poisson) 2. Lates niloticus perche du Nil

1540 sàà $\boldsymbol{n}$ (contr) 1. natte 2. surface plate (partie plate de la surface)

1541 saa $n$ Setaria barbata graminée sauvage (esp.)

1542 sáá rn groupe, attroupement (qui vont à la file, à la queue leu leu, en file indienne) $\diamond$ zro sáá ramassis des fourmis $\square$ ò sáá nwâ ý ta il sont venus nombreux chez moi

1543 saade $\boldsymbol{n}$ Thrasops occidentalis serpent noir arboricole

1544 sáánle $\boldsymbol{v}$ dépiquer, battre $\square$ ò yàa mlò saan yri lé sò avant on battait le riz avec des bâtons

1545 sáánni bhe adv tout de suite $\square$ ké ká kpá lòo mu lé droón ó yáa ká delè sáánbhe mais si vous êtes des Gouros, nous allons vous tuer tout de suite

1546 saanwli <Dioula> $\boldsymbol{n}$ mode $\square$ ý yóo saanwli so mu lòlé je veux acheter des pagnes à la mode

1547 sààwóle $\boldsymbol{v}(\mathrm{mob})$ annuler, abandonner $\square$ ò ge za wàla ć sáàwòa leur départ pour le village est annulé

1548 saflée $\boldsymbol{n}$ savon (traditionnel) $\diamond$ saflée ti savon noir

1549 sàkósì <Fr. sacoche> $\boldsymbol{n}$ sac

1550 sàkla $\boldsymbol{n}$ peigne (de type africain) $\square$ Syn. wíngope 
1551 sàládì $<F r$. salade $>\boldsymbol{n}$ salade (plant)

1552 sàmá $<$ Dioula $>\boldsymbol{n}$ éléphant

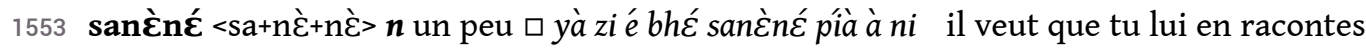
un peu

1554 sánigbòo $\boldsymbol{n}$ ganglion enflammé

1555 sánige <Dioula> conj 1. avant que $\square$ sánni ó nu gele, ó pebhlà pé avant de partir nous avons mangé 2 . au lieu de $\square$ é yáà so bhole ý ba sánnì é nu é gbú lii gliinle dwàtéy yí tu aurais dû m'aider au lieu de te regarder dans le miroir

1556 sánlè $\boldsymbol{v}(\mathrm{mob}) \mathbf{1})$ vi grimper, monter $\square$ wòo san yrì yí le singe grimpe à l'arbre $\nabla$ sánle sóo ta monter sur un vélo 2) vt trébucher $\square$ ké $m \varepsilon \varepsilon$ gbe kòo ta gaan sààn kpoti ma, bhé kle zàblé láá ò à lèc si ton pied gauche cogne un caillou, c'est qu'il n'y a pas de bonheur devant toi

sánle $\boldsymbol{v}(m o b)$ 1) vt commencer (à faire qqch - wóle) $\square$ gwláan ś so lùa à na É do ni bhéc̀ à na vìndomi zran wole sààn l'homme a acheté un pagne à une des ces femmes, et l'autre a commencé à être jalouse $\diamond$ ké è sán depuis $\square$ ké è sán ý le yaláá, náá ý núàgwlecnnè yéle depuis que je me suis marié, je n'ai pas vu mon petit frère 2) vi commencer $\square$ ye $\varepsilon$ san zrwanzi le travail commence le matin sánnì, sánnî́ $a d v$ (contr) 1. en ce moment, maintenant, actuellement $\nabla$ sánnì yromazi plé jusqu'au soir (lit. entre maintenant et ce soir) 2. tout de suite

1559 sánygansó <Dioula sánkanso> $\boldsymbol{n}$ bâtiment à étages $\square$ Syn. táási

1560 sánsi $<$ Fr. essence> $\boldsymbol{n}$ essence $\diamond$ sánsi gblè غ̀n odeur d'essence

1561 sayye $<$ Dioula sánke $>\boldsymbol{n}$ mousticaire

1562 sasa det un peu, un tout petit peu (Afr.) $\square$ jàn É wó sasa parle un peu $\square$ ǵ súglùu tòo $\varepsilon$ ́ sasa bhlà j'ai mangé un peu de ce tô de manioc

1563 sàwlà $<$ Arab. sabbaat. ou Port. sapata $>\boldsymbol{n}$ chaussure

1564 sàyaàn $<$ Fr. assaillant $>\boldsymbol{n}$ assaillant

1565 se $\boldsymbol{n}$ graine de palmier à huile (dont la pulpe est comestible) $\diamond$ se ýgblo régime de graines de palmier à huile

1566 séé $a d v$ absolument, complètement (intensif pour kpén) $\diamond m \varepsilon \varepsilon$ kpén séé tout le monde sans exception

1567 séébáá $<$ Dioula $>\boldsymbol{n}$ jeune femme

1568 sékéséké $a d v$ jamais plus (intensif à li pas du tout) $\square$ náá nu pebhlele li sékéséké à ba je ne mangerai plus jamais chez lui

1569 sélí $\boldsymbol{n}$ prière $\diamond$ séliwóle prier (pratique religieuse de toute religion) $\square$ kذ̀ó kذ̀ó séli wó prions ! 口 ráábi mu séli wo gbeya ò bhîi bamu les arabes prient comme les Dioulas (c'est-à-dire ils sont musulmans)

1570 selilk $\boldsymbol{\varepsilon}<$ Fr. cellulaire $>\boldsymbol{v}$ téléphone portable

1571 sélíwófé <sélí+wo+fé> $\boldsymbol{n}$ église $\square$ Syn. leglizi

1572 seluu bòn $\boldsymbol{n}$ Mariscus alternifolius herbe (esp.)

1573 seluu $\boldsymbol{n}$ déchets des graines de palmier à huile (les fibres des graines de palmier après l'extraction de l'huile de palme)

1574 semúù $\boldsymbol{n}$ crevette 
1575 sénsén $\boldsymbol{n}$ hoquet $\diamond$ sénsén bole donner le hoquet

1576 séy $<$ Dioula>n 1. pont (en bois) จ séygboo pont 2. capacité, possibilité

1577 sènsèý adv ideo sur un pied (marche de l'unijambiste)

1578 séywóle $\boldsymbol{v}$ vaincre, surmonter, avoir le pompon $\square$ Táátoo séy wóa Tóonc lé Tato a surmonté Toné

1579 Sètrán $<$ Dioula $>\boldsymbol{n}$ Christ. Satan, diable

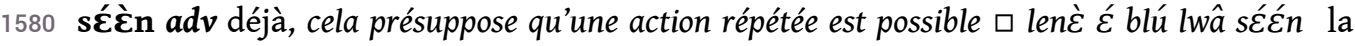
femme a déjà acheté du pain

1581 sहยn onomat chirrr (son émis par le grillon)

1582 sर́์ $\boldsymbol{\varepsilon} \mathbf{\varepsilon} \boldsymbol{n}$ petit carnassier (chacal, mangouste, chat)

1583 sćḱ $\mathbf{b}$ potasse [ajouté à la sauce pour la rendre claire; est produit de la cendre de pelures de cacao ou de bananes brûlés]

1584 sर́nné, sćlén $\boldsymbol{n}$ racine (d’une plante)

1585 sćtí <Fr. assiette> $\boldsymbol{n}$ assiette

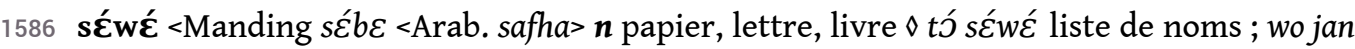
sÉwé yì manuel ; Christ. Zèzù séwé: Bible

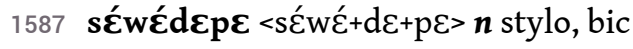

1588 sì $<$ Fr. scie> $\boldsymbol{n}$ scie

1589 sí $\boldsymbol{r n}$ clan, lignée, tribu $\diamond$ síya clanisme; sí mu parents, membres de la même tribu, famille

1590 sì $r n$ vie $\square$ ké yéé de mu klòsì kwàn, si gbojn ò é kóó si tu respectes tes parents, tu auras une longue vie

1591 sian $\boldsymbol{n}$ Capra ibex bouquetin, mouflon, cabri de brousse

1592 siánle $v(m o b)$ 1) $v r$ éternuer [quand quelqu'un éternue, d'autres prononcent son nom clanique; si quelqu'un éternue à droite c'est un bon signe, éternuer à gauche est un mauvais signe] $\square$ yée siáánlà tu as éternué $\square$ mí ý siáánlà klèklè ć kóó le piment m’a fait éternuer 2) $v t$ faire éternuer

1593 sie $\boldsymbol{n}$ fouet, chicotte (Afr.)

1594 siésíé gàma $\boldsymbol{n}$ sauterelle (esp.)

1595 sićle $\boldsymbol{v}\left(\right.$ mob) 1) vi 1. se gâter 2. se casser (y compris des appareils électromécaniques) $\square$ f́́ $\mathcal{\varepsilon}^{-}$

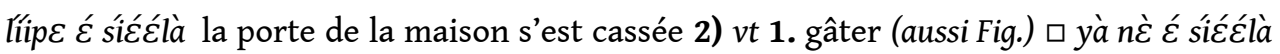

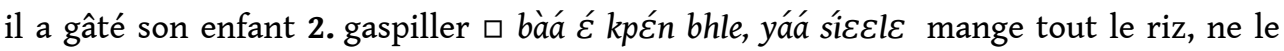
gaspille pas

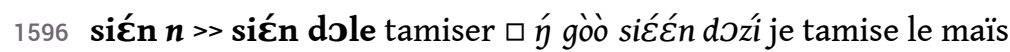

1597 sićn $\boldsymbol{n}$ côtes $\diamond$ siććnbhelé côte $\square$ ý sićnbhelé do takààn je me suis enfoncé une côte

1598 síí adv 1. peut-être, sans doute $\square$ ò yàa wèzí síi ká mu là lè sans doute, ils parlaient de

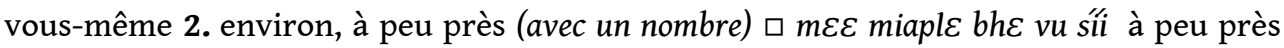
cinquante personnes

1599 sî́le v 1. vt appeler (par le prénom) $\square$ wò ý sii Móisí je m’appelle Moïse $\square$ yà gbe Gemi sìilà il a appelé son fils Guémi 2. inviter (chez - ba) 3. téléphonner

1600 síin $a d v$ auparavant 

dommage !; tákísí síle prendre le taxi 2. porter, emporter $\square$ nu bho ý ba cie síle lé viens m'aider à porter le panier $\diamond$ à gbeya síle copier la manière de qqn de faire qqch $\square m \varepsilon \varepsilon$ tááwógbeya síle copier la démarche de qqn 2) vi 1. s'emporter $\square$ yáa fáá é sía é ǵgbhlo ma ton chapeau s'est envolé de ta tête 2 . voler, s'envoler (d'oiseau) ฉ Syn. tánle

1611 sòófàlí $<$ Dioula $>\boldsymbol{n}$ 1. Equus asinus âne 2. cheval

1612 sóóle $v$ mâcher

1613 sóómi adj cinquième

1614 sóyonlima $<$ Dioula> $a d v$ accroupi $\diamond$ o sóyonlima être accroupi $\square$ ò yaàzí ò sóyonlima té $\varepsilon$ $z i$ ils sont accroupis à côté du feu

1615 sò $a d v$ 1. autrefois, auparavant $\square$ sò, ké bhlèggbè é yáà nu yre lá noj̀ mo kpén yáà nu à púbole jadis, quand le roi arrivait, tout le monde venait le saluer 2. en attendant $\square p \varepsilon \varepsilon$ ́́ ké bhlé sò ýóo zan mange en attendant, j'arrive

1616 so $n$ 1. tissu $\diamond$ sotànmi tisserand [traditionnellement, parmi les Mwans il n'y pas de tisserands, le tissage est généralement fait par les Dioula] 2. pagne [pièce d'étoffe ou de matière végétale tressée que l'on porte drapée autour des hanches et qui couvre le corps de la taille jusqu'aux mollets]

1617 sóón $\boldsymbol{r n}$ totem [un animal ou une plante considéré comme le sauveur de l'ancêtre du clan,

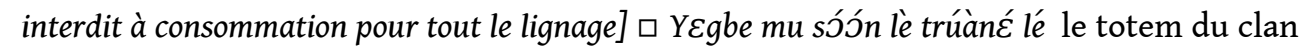
Yegbé est la panthère

1618 sòònnî́i $\boldsymbol{r n}$ ennemi

1619 sòònsòòn $\boldsymbol{n}$ épilepsie $\square$ sòònsòòn ò nè É ýi l'enfant a l'épilepsie

1620 SJgb $\boldsymbol{n}$ morceau de tissu

1621 sóle $\boldsymbol{v}$ 1) vt planter (plante) $\diamond$ sóle éke aplanir, niveler 2) vi être planté

1622 sóle $v$ 1. vi être aimé, plaire $(\grave{a}-n i) \square n \varepsilon \grave{\varepsilon} m u$ so ò $n \varepsilon \varepsilon$ ni la mère aime ses enfants tááwále bhlù wee ta laa so à ni la vipère n'aime pas qu'on marche sur sa queue 2. atteindre (qqn-ma)

1623 sóle vi 1. pouvoir $\square$ ý so yi kánlé je sais nager 2. devoir

1624 sólè vi être bien formé (bananes, concombres, maïs, manioc et autres plantes qui ne changent pas de couleur, qui sont mangés non mûrs, mais quand ils ont atteint une certaine taille) $\square$ blaán É sóle ò la banane est formée 
1625 són $\boldsymbol{r n}$ dent, défense $\diamond$ són bhe une dent; bie són bhe défense d'éléphant; són kpále mordre, piquer ; són dele (se) noircir les dents ; són yáánle tailler les dents ; són síle rire

sònmo $n$ Anacardium occidentale 1. anacardier, cajou [arbre tropical] 2. anacarde (noix de cajou)

1627 sònsî́ <sòn+sí> $\boldsymbol{n}$ rire

1628 sónsíle <sòn+síle> vi 1. rire 2. se moquer $(d e-m a)$

1629 sòý $n$ mutilation permanente, infirmité, défaut physique $\square$ sòý lá bhé ò à ma, bhéle ké à gbe gale lé son infirmité est au niveau de son bras qui ne fonctionne pas

1630 soy $\boldsymbol{r n}$ cœur [pour les Mwans c'est un organe sans grand importance; on croit que c'est le foie qui est le centre des caractéristiques spirituelles d'une personne] $\square$ ý soy ć yéle la ý kpéé c'est mon cœur qui me fait mal

1631 sotanmi $<$ SO+tan + mi $>\boldsymbol{n}$ tisserand

1632 srà n payement, salaire $\square$ yà klà à srà kpá za yí il l'a fait pour être payé

1633 sra $n$ querelle, dispute $\diamond$ sra dole faire querelle, disputer; sra dole à ma gronder

1634 sráále $v$ 1) vt 1. piquer (qqn, qqch) 2. coudre [occupation des hommes de préférence] $\diamond$ sosràmi, sosráámi tailleur 2) vi 1. piquer (piment, moutarde), être ardent (soleil) 2. être méchant (personnes) 3. (seulement Prf) être cousu

1635 sràbà <Dioula> $\boldsymbol{n}$ mèche (de lampe à pétrole)

1636 sráká <Manding sáráká <Arab șaḍaqa> $\boldsymbol{n}$ offrande $\diamond$ sráká gole sacrifier (que - lé)

1637 sràn $\boldsymbol{n}$ grenouille $\square m \varepsilon \varepsilon$ bhغ̀c̀ké mu sràn bhle certains gens mangent des grenouilles

1638 sranغ̀ $\boldsymbol{n}$ tisserin (oiseau) [oiseau passereau, capable de tisser un nid suspendu]

1639 sráygo n Combretum micrantum quinquéliba (plante médicinale)

1640 srázé $<$ Fr. chargement $>\boldsymbol{n}$ chargement

1641 sre $\boldsymbol{n}$ poivre, gingembre, piment souterrain

1642 sréć rn groupe, troupeau $\square$ dri sréć $\varepsilon$, wò yí mízíi drà $\varepsilon$ ba le troupeau de vaches boit l'eau du lac

1643 sréć rn 1. descendant (par le lignage du père, par lequel les tabous alimentaires sont transmises) $\square$ Tóo sréć mu descendants de Too $\diamond$ sréć do un descendant $\square$ zòn yi lèc̀ ma ómoว Zèíibàa sréć mu trúànć bhle, wáá trúànć to li de nos jours, nous, famille de Zeiba, mangeons la panthère, nous ne nous en abstenons plus 2. témoin (au jugement) $\diamond$ klele sréćlé témoignage

1644 sreygbete adv ideo mince, fin

1645 srii $\boldsymbol{r n}$ tristesse $\diamond$ sríiwóle être triste $\square$ mèlàma é wle yì sríiwóle ò ćc̀ ? pourquoi es-tu si triste?

1646 srínle $\boldsymbol{v}(\mathrm{mob}) \mathbf{1})$ vi rester pétrifié sur place, rester cloué sur place $\square$ è jàrà gbèntèn $\varepsilon$ yéle ké è sriplé gblàn ć kóó il a vu un gros lion et il était pétrifié par la peur 2) vt pétrifier sur place, clouer sur place $\square$ jàrà gbinnàle $\varepsilon$ $m \varepsilon \varepsilon$ É sríylà le rugissement du lion a pétrifié l'homme

1647 sró $\boldsymbol{n}$ chanson, danse $\diamond$ srókpále chanter ; srótánle danser $\square$ sróó kpále pégeé sróo tánle so à ni il aime chanter et danser

1648 srò $\boldsymbol{n}$ sifflet $\diamond$ srò pénle siffler

1649 sròmà $\boldsymbol{n}$ pardon $\diamond$ sròmà bole demander pardon ; $m \varepsilon \varepsilon$ a sròmà yánle pardonner à qqn 
1650 sróóle $v$ 1) vt oindre, frotter (avec - lé) $\square$ ò gademi ć sróóla ké kwa lé on a frotté le malade avec du kaolin 2) vr se pommader, se frotter [traditionnellement on frottait le corps avec de l'huile de palme ou du beurre de karité (surtout les femmes et les enfants)]

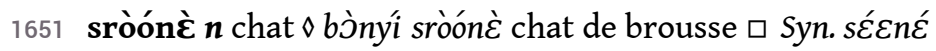

1652 srò $\boldsymbol{n}$ ongle, griffe

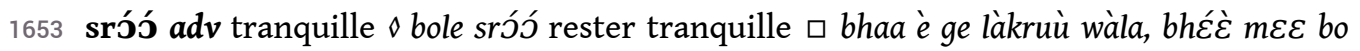
sróó qu'il aille à l'école et que les gens aient la paix ; tóle sróó laisser tranquille $\square$ gbàan ع́ tó sró laisse le chien tranquille!

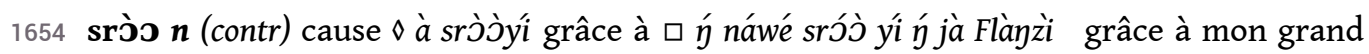
frère je suis allé en France

1655 sróónnî́i $n$ sorcier $\square m \varepsilon \varepsilon$ dele sว sróónníi $\varepsilon$ bhé $\varepsilon$ ni les sorciers aiment tuer les gens

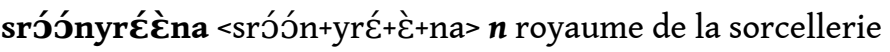

1657 srón $\boldsymbol{n}$ sorcellerie

1658 srónníí moo $\boldsymbol{n}$ Sorcidae, Rhynchocyon cirnei musaraigne, rat à trompe à damier [la souris-sorcier, on ne la mange pas]

1659 SrOpe n 1. Panthera pardus panthère $\square$ Syn. trwânć 2. carnassier

1660 srùòwóle, sròòwóle $\boldsymbol{v}$ (contr) 1. vt gagner, trouver, avoir (argent, lettre, cadeau etc.) $\square \dot{y}$ jà ye bhéċ mí án pe glaán sròj̀wòa je suis allé là-bas et j'ai reçu mon cadeau 2. récolter $\square$ kذ̀ó súgrùú bèbè sròj̀òa nous avons eu une bonne récolte de manioc 3. gagner (à lotterie) $\square$ nc̀ lá míliyón vu ta sóó srój̀wóa yélà yaàlele bhe l'enfant qui a gagné quinze millions est assis ici 4. réussir (dans)

1661 srú $\boldsymbol{n}$ forêt (près d'un cours d'eau)

1662 srùngbhoó $\boldsymbol{n}$ foulard

1663 sróa, srúa num huit

1664 sróami, srúami num huitième

1665 sróado, srúado num six

1666 sróadomi, srúadomi num sixième

1667 sróaple, srúaple num sept

1668 sróaplemi, srúaplemi num septième

1669 srùàsé $<$ Dioula sòrJdasi $<$ Fr. soldat $>$ n soldat, militaire

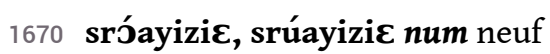

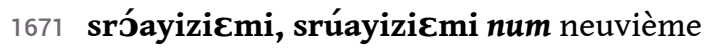

1672 sú $n$ cure-dent [un bois mâché destiné au nettoyage des dents] $\diamond$ sú gòj̀le se nettoyer les dents avec un cure-dent $\square$ ý sú $\varepsilon$ glò bhéċ án kan ý són ta je mâche le cure-dent et je le passe sur mes dents

1673 súà $\boldsymbol{n}$ cochon, porc

1674 súáá adv ideo lentement

suaan $a d v>>$ tén suaan jaune

súàgblèèmi $n$ éleveur de porcs

1677 súéle vt guérir miraculeusement [par exemple, par aspersion d'eau] 
1678 sùè $\boldsymbol{n}$ Mormyrus macrophthalmus mormyre (poisson électrique d'eau douce)

1679 súékò $\boldsymbol{n}$ Civetticis civetta civette (d'Afrique)

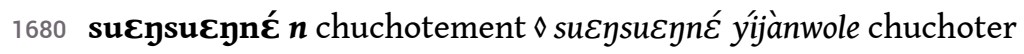

1681 súglùú $\boldsymbol{n}$ Manihot glaziovii manioc $\diamond$ súglùu kpáciéle manioc braisé ; súgluuu pon foutou de

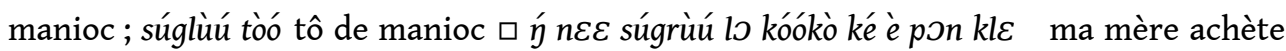
du manioc pour faire le foutou

1682 sukalo, sukaro $<$ Fr. sucre $>$ n sucre

1683 suluu adv ideo face à face

1684 súmawédèn $\boldsymbol{n}$ fétiche d'eau (nom d'un certain bassin naturel) [adoré par le clan de Yegbé] $\square$ ò súmawédèy gbale maa lé c'est avec le poulet qu'on adore le Sumaweden

1685 súy $n$ jeûne $\diamond$ súnwlále jeûner

1686 súymle $<$ súy+mle> $\boldsymbol{n}$ Ramadan

1687 susulusu adv ideo insensible, engourdi

1688 suù $<$ Fr. choux $>$ n chou

1689 súúsù $a d v$ habituellement

\section{$T-t$, comme tàà, táátáánć, tàbàlí}

1690 ta pstp 1. sur, à $\diamond$ tàbàli ta sur la table 2. à cause de $\diamond$ mò ... dàwliya ta survivre par la ruse 3. en numeraux $\diamond$ vutado onze

1691 taà conj ou, ou bien $\square$ è nc̀ lá yáà, gwláan lè taà le lè ? de qui est-ce qu'elle a accouché, d'un garçon ou d'une fille?

1692 tàà $\boldsymbol{n}$ (contr) 1. $r n$ grand père, aïeul $\diamond$ à tàa $m u$ ces grands-parents 2 . ancêtre

1693 táá n 1. marche, voyage $\diamond$ tááwóle marcher; tááwó yri canne; tááwó gbeya comportement, coutume 2. fois (des actions homogènes) $\diamond$ táá léċ ýi cette fois-ci

1694 Táá nránle <táá+nrále> intrj bon voyage !

1695 tááyíí, táîy toujours $a d v$

1696 táásì adj à étages (bâtiment) $\square$ Syn. sángansó

1697 taataa $a d v$ ideo vite-vite $\diamond$ à gbe ta ò taataa il est généreux

1698 táátááné n Citharinus citherus poisson-lune

1699 tàbàlí, tabalí <Fr. table $>\boldsymbol{n}$ table

1700 tabasakîi $<$ Dioula $>\boldsymbol{n}$ tabaski

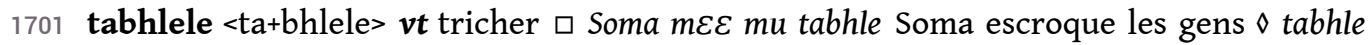

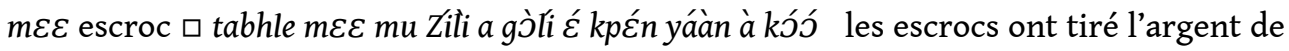
Jules

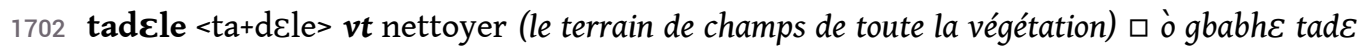
pé bhéc̀ ò fe pa on fait le débroussaillage du terrain avant de faire les buttes

1703 taféle $<$ ta+fele> vt Fig. casser la gueule à qqn, frapper $\square$ ýé tafe bhéc̀ zàké láo à yi je te casse la gueule certainement

1704 tàfò <Dioula> $\boldsymbol{n}$ amulette de tissu (de bras ou de pied; fait de tissu, ou de fil de coton) [il est utilisé pour les danses rituelles, comme amulette pour les personnes malades et fétiche 
protecteur pour les enfants] $\diamond$ táá wó tàfo anneau de marche $\square$ ò táá wó tàfo bwà nغ̀ é gaan ma on a mis un anneau de marche au pied de l'enfant

1705 tagaanle $<$ ta+gaanle> vt protéger qqn (empêcher qqn d'être exposé à un danger; de - lècे) $\square$ le à nòò tagàànnà fáázan mu lèc̀ la femme a protégé son mari de la police

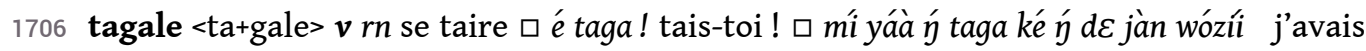
l'habitude de me taire quand mon père parlait

1707 tagbenle $<$ ta $+b \varepsilon n l e>v t$ attendre (un peu) $\square$ ý tagben ! attends-moi !

1708 tagbìnle $<$ ta+gbinle $>\boldsymbol{v}$ surveiller $\diamond$ tagbinmi surveillant, Christ. évêque

1709 taglòòle <ta+glòj̀le> $v t$ essuyer, époussetter $\square$ tàbàli é taglòo so kple lé essuie la table avec le chiffon

1710 taglinle $<$ ta+glinle $>\boldsymbol{v} 1$. $v$ t surveiller, observer qqch, qqn (avec attention: la sauce, l'enfant qui dort etc) 2. visiter, rendre visite $\square$ Zonma pégée Soma nwà ò de mu taglinle zòn Zonma et Soma sont venus rendre visite à leurs parents aujourd'hui

1711 tagole $<$ ta+gole $>\boldsymbol{v} \mathbf{1}$. nettoyer (une surface plate, comme la table) $\square$ tàbàli $\varepsilon$ tago! nettoie la table !2. vi maigrir $\square$ à tagwà ga $\varepsilon$ kóó elle a maigri à cause de la maladie

1712 takánle <ta+kánle> vt 1. traverser 2. couper $\square$ Po ò yj̀ò gboo É takánzíi poo É lé Po est en train de couper le fagot avec la hache $\diamond$ yo takánle extraire le vin de palme 3. défricher $\square$ ý maza ò é ma gba é yòj̀ ć takán za yí j’ai besoin de toi pour défricher le champ

1713 táklá $<$ Dioula $>\boldsymbol{n}$ allumettes $\diamond$ táklábh $\varepsilon$ allumette

1714 takláále <ta+káále> vi se calmer (diminuer d'intensité; phénomènes naturels) $\square$ la É ta ò kláázíi la pluie est en train de se calmer

1715 taklóale, takwláále $<$ ta + kwláále $>\boldsymbol{v}$ perdre du poids (un peu) $\square$ à taklwààlà il a perdu du poids

1716 takpáále <ta+kpáále> $\boldsymbol{r v}$ se courber (en passant sous qqch) \ mí ý takpàláá bhéc̀ ý káàn bhleklo ć lâ je me suis courbé pour passer sous la liane

1717 takpále <ta+kpále> vt ajouter (au marché), donner (ration supplémentaire) $\square$ án mlò $\varepsilon$ takpá ! ajoute-moi du riz !

1718 takulàle <ta+kulàle> vi s'abaisser (en passant au-dessous de qqch) $\square$ bhlekJo ò bj̀j̀ ć yí ébèbè lé kà ká takulà bhéc̀ ká kan il y a beaucoup de lianes dans la forêt, abaissez-vous pour passer

1719 tàmatá $n$ pipe $\square \grave{y} d \varepsilon$ yàa tàmatá mi mon père fumait la pipe

1720 tami $\boldsymbol{n}$ l'aîné, le supérieur (l'enfant le plus âgé de la famille)

1721 tàn $\boldsymbol{n}$ tresse

1722 táàné >> jàn ma táàné $\boldsymbol{n}$ devinette [au village on posait des devinettes les soirs après les travaux de champs] $\diamond$ táànć bole poser une devinette

1723 tánle $\boldsymbol{v} \mathbf{1}) v t$ 1. tresser (cheveux, panier, corde) $\diamond$ yrèlíi tánle fermer les yeux; sró tánle danser $\square$ ò sró tan fÉti yí on danse pendant la fête 2. tisser (lacets, corde) 2) vi être tressé

1724 tánle $v t$ 1. marcher (sur - ta) $\square$ Bie tààn Súa a kpòo ta Éléphant a piétiné la houe de Porc 2. écraser (d'une voiture; qqn, qqch - ta) $\square$ gòn ć tààn bhlà da $\varepsilon$ ta la voiture est passée sur le mouton 3. donner un coup de pied (à qqn, qqch - ma)

tánle $v t$ enterrer (tubercules) $\square$ ké ò bhé yaan là tánzí et voilà qu'ils plantent de l'igname 
1726 tànga $<$ Dioula tànká ' 50 centimes'> $\boldsymbol{n}$ pièce de 20 centimes

1727 táygófáá $\boldsymbol{n}$ Atractaspis vipère creusant

1728 táyîí, táín $a d v 1$. toujours $\square$ è yàa sìklèti mi táî́ il a toujours fumé $\square$ gwláan léc̀ nu sóle à lù ni táyíi sa fille aimera toujours ce garçon 2. un jour (dans des propositions conditionnelles)

1729 tape $n$ 1. couverture (tout ce qui peut se mettre sur qqch) 2. toit

1730 tapele $<$ ta+pele> $v t$ lire $\square$ Pićli pe Zàle ni è sÉwÉ tape Pierre dit à Zalé de lire sa lettre

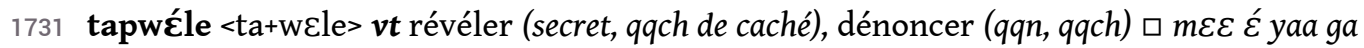
jàn É tapwèlà cette personne a annoncé la nouvelle de sa mort $\square$ Po nule ké Gbayro LĖgè tapwélé à gaanyre nò̀ quand Po est venu, Gbayro a dénoncé la cachette de Lengué $\diamond$ zà tapwéle révéler qqch

1732 tásá $<$ Dioula $>\boldsymbol{n}$ cuvette

1733 tásì $<$ Fr. tasse $>\boldsymbol{n}$ tasse

1734 tasíle $<$ ta+síle $>\boldsymbol{v}$ être bête (ne pas connaître toutes les règles de la communauté, les choses les plus élémentaires) $\square$ Tátà kla zà kpén yre ba, è we jàn kpén ma bhéć é jàn kpén yílróò kpa, bhÉyaćyí à tasíle ò Tata se mêle à tout, elle s'invite dans les causeries et pose des questions sur toutes les choses, en effet, elle est bête

1735 tàtabhíà $<$ Dioula tàsabiya $>\boldsymbol{n}$ chapelet, rosaire

1736 tatróóle <ta+tróóle> vi grandir, croître (enfants, jeunes plantes ou animaux; en taille) $\square$ $n \dot{\varepsilon}$

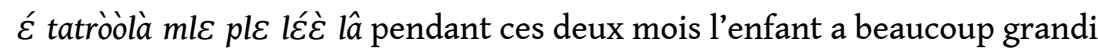

1737 tayròòle $<$ ta+yròòle> $\boldsymbol{v}$ passer à travers un filtre, filtrer (liquide avec du tissu propre) $\square$ mwà $m u$ wàa lòmlin yí tayroo tèmè yí les Mwans filtrent le jus d'orange avec un tamis

1738 tazonle <ta+zonle> vt nettoyer (le champ nouveau où la brousse est déjà brûlée) $\square$ Táatóo ò gbabhe tazonzi Tato prépare un nouveau champ

1739 té adv exactement (temps, argent, quantité et mesure), seulement $\square$ gòli vu na ý ni té donne-moi exactement cinquante francs $\square$ ý maa yaga té ye je vois exactement

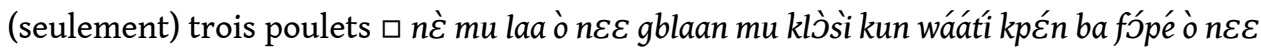
$m u$ té les enfants n'obéissent jamais à leurs grand-mères, ils n'obéissent qu'à leurs mères

1740 te $\boldsymbol{n}$ 1. cataracte (affection de l'œeil caractérisée par une opacité totale ou partielle du cristallin) 2. malheur

1741 téètéè intrj hélas (exclamation exprimant une plainte ou un regret)

1742 tèètéé, téétee adv ideo clignant (des yeux à cause de la lumière vive)

1743 tèlèvìziòn $<$ Fr. télévision $>\boldsymbol{n}$ télévision, téléviseur

1744 téy $a d v$ exactement, précisément, justement (position, disposition) $\square$ án fé $\varepsilon$ yò̀ Bàànluma wà ć dreyí tén ma maison se trouve exactement au centre du village de Bambalouma $\square$ f́́ $m u$ É wò̀ éke téy ta les maisons sont alignées soigneusement

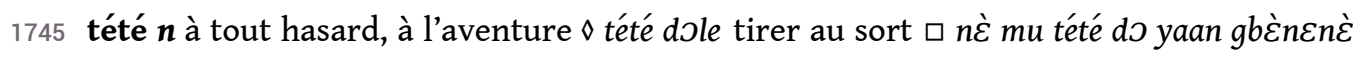
$m u$ ta les enfants font des tirages au hasard sur les petites ignames

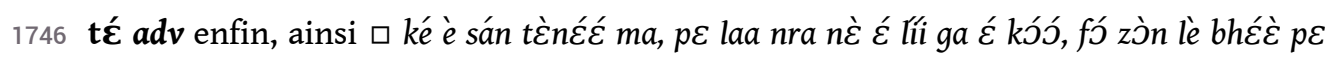
nràlà tỉi té depuis lundi l'enfant n'a pas eu d'appétit à cause de sa maladie, c'est aujourd'hui enfin qu'il a de l'appetit 


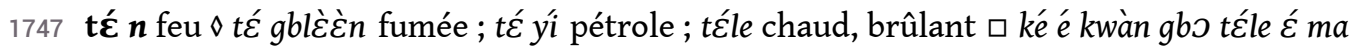
yée gbe ma blo wo si tu saisis le canari chaud, tu vas te brûler les mains; té bole allumer le feu; té bole bon yí feu de brousse (pour la chasse); t té bhíle lumière; té wlále incendie

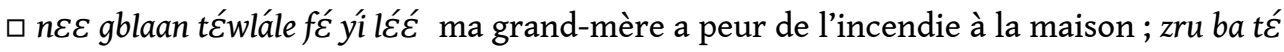
ò s'inquiéter

1748 Té ta bhla zi $\boldsymbol{n}$ janvier (temps de la chasse avec le " feu de brousse »)

1749 tébhléle $<$ tćtbhlele $>\boldsymbol{v}$ brûler

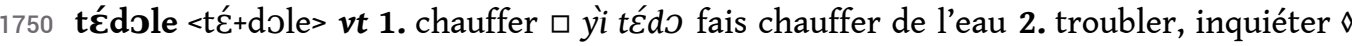
zru ba tédole donner sur les nerfs ; yrغ̀ ba tédole ennuyer, embêter

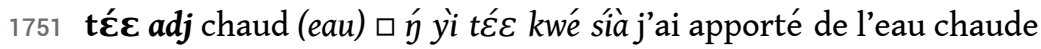

1752 tè̀े $\boldsymbol{n}$ rancune

1753 té غ̀ part c'est intéressant (marque de question générale avec une nuance de doute) $\square$ potáblù léc̀ $y$ nu sole à magaanlè téc̀ ? c'est intéressant, est-ce que je saurai réparer ce portable?

1754 téfúú <t<́+fúú> $\boldsymbol{n}$ chaleur $\square$ téfúú $\varepsilon$ kóó ó bwà fé lé ma à cause de la chaleur nous sommes restés à la maison

1755 tégblè ̀̀n $\boldsymbol{n}$ fumée

1756 téle adj chaud $\square$ zru gblan láá bàá téle bhle gros cœur ne mange pas de riz chaud (proverbe : même si tu es courageux, tu ne peux pas manger de riz chaud)

1757 t ṫ̀m $\boldsymbol{\varepsilon}<$ Fr. tamis> $\boldsymbol{n}$ tamis

1758 tén adj rouge (inclut tous les nuances de rouge, orange, jaune et brun clair) $\diamond$ tén klèćn rouge vif; tén fláóflao orange, jaune ; tén jilà̀ brun clair

1759 tèné, tènné <Dioula> $\boldsymbol{n}$ lundi $\diamond$ tènć $\varepsilon$ $m a$ le lundi

1760 tènén $\boldsymbol{n}$ insecte (esp.)

1761 ténkpále $v$ vr s'adosser (à - yí) ロé ténkpá yrì diin é yí adosse-toi à l'arbre

1762 ténle v 1) vi 1. rattraper (qqn - ma) $\square$ wáá sJ ténle Zàle ma nous ne pouvons pas rattraper Zalé 2 . arriver, se passer $(\grave{a}-m a) \square$ zà yóo gbèntèn do tiàn à ma il lui est arrivé un grand malheur 3. atteindre (aussi pour l'âge) (qqch - ma) 4. s'adosser (à - yí) $\square m \varepsilon \varepsilon \varepsilon$ ́́ tÉnle ò gble diin ć yí à na magben yre nò̀ l'homme s'est adossé au baobab en attendant

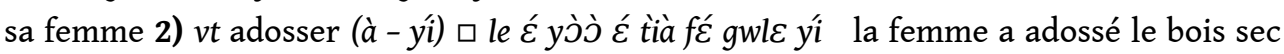
au mur

1763 tépléỳ $\boldsymbol{n}$ lieu du feu de brousse, brousse brûlée

1764 tétriîin, tétíni $\boldsymbol{n}$ charbon (de bois) प tétríin bo zièn é ba bhéċ à tón láá do mets du charbon dans la sauce pour qu'elle ne se gâte pas

1765 téyóòn $\boldsymbol{n}$ braise

1766 ti adj noir, sombre (inclut les tons sombres des toutes les couleurs, ainsi que les couleurs froides : bleu, vert, gris) $\diamond$ ti yrèyrè vert ; ti kpélikpéli noir ; yrèti pupille (d'œil)

1767 ti $\boldsymbol{r n}$ (contr) oncle maternel, oncle (Iv.) (frère cadet de la mère; par rapport au neveu drùanè) [il a des relations spéciales avec son neveu, le fils de sa sœur: le neveu travaille sur le champ de son oncle, mais est également autorisé à prendre n'importe quelle chose qu'il aime dans la maison de son oncle] 
1768 tiin $a d v$ soigneusement $\square$ Bhoo á yrćkpáa yo mu ć ba tiin Cabri a examiné les fétiches avec minutie

tilàle v 1) vi noircir, devenir noir $\square$ nè $\varepsilon$, yòo tilàzí cet enfant est en train de noircir 2) $v t$ rendre noir $\square$ yrèté $m \varepsilon \varepsilon$ tilá le soleil fait noircir les gens

1770 tìsà $\mathbf{n}$ Cassia sieberiana casse du Sénégal, casse de Siéber, casse flûte

1771 tìtì $a d v$ du tout, jamais $\square m \varepsilon \varepsilon$ gwe là ò ba titì il n'y a aucun vieux parmi eux

1772 to $\boldsymbol{n}$ roseau

1773 tòà $\boldsymbol{n}$ Galago $\mathbf{s p}$. galago

1774 toko $n$ Herpestes ichneumon, Crossarchus obscurus mangouste (on peut appeler comme ça un autre petit prédateur, une civette ou une genette)

1775 tóle vt 1. laisser (avec intention) $\square$ Màrí kàkàó bhaa é tóa gè bhéc è jilàa Marie a laissé les cabosses de cacao ici et elle est partie $\square$ ò pebhle vì to $n \varepsilon \grave{~} m u$ ni on laisse les restes de nourriture aux enfants $\square$ à tó sró laisse-le tranquille $\diamond m \varepsilon \varepsilon$ ma za tóle pardonner à

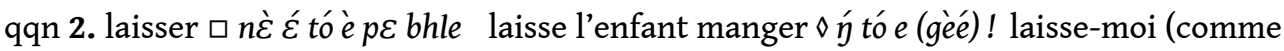
ça)! 3. Animist. s'abstenir de [habituellement cela suppose de ne pas manger certains aliments, mais aussi de s'abstenir, par exemple, de porter de choses en or] $\square$ Gòge mu ć mú yáa mlò to les Gogbé ne mangeaient pas du riz

1776 tólíi $<$ Fr. tôle $>\boldsymbol{n}$ tôle $\diamond$ tóli fé la maison à toit de tôle

1777 tóy $\boldsymbol{n}$ loi (traditionnelle) $\diamond$ tónta za règles de la vie traditionnelle $\square$ tónta za é séy ò $m \varepsilon \varepsilon m u$ É kpén ni respecter la loi est obligatoire pour tout le monde ; tón dulàle établir la loi

1778 too $n$ 1. jour (opposé à nuit) $\diamond$ too klinle arriver (jour), faire jour $\square$ bhé klele bhé too $\varepsilon$ klíinlà tout était comme ça et le jour est arrivé 2. demain $\square$ too kòó nu gele gba la demain nous irons au champ $\diamond$ lec too l'année prochaine

1779 too $\boldsymbol{n}$ espace derrière qqch $\diamond f \varepsilon$ too zi derrière la maison

1780 tòó $\boldsymbol{n}$ tô (pâte de farine de maïs ou de manioc, ou de leur mélange)

1781 tóo $r n$ homonyme

1782 tòòló $\boldsymbol{n}$ avertissement, annonce (annoncé par un crieur publique au village) $\diamond$ tòolódole crier

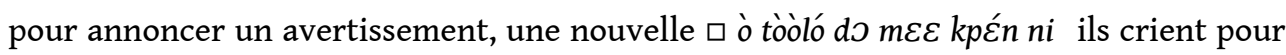
annoncer un avertissement pour tous ; tòolódo sćwé journal, magazine

1783 tòòlódomi <tòòló $+\mathrm{d} 0+m i>\boldsymbol{n}$ crieur public, héraut

1784 tootama $a d v$ après-demain

1785 toozi $n$ alentours (de la maison), abord (de la maison) $\square$ f́́ toozi mu $\varepsilon$ trîin ò le passage à la maison est remplie d'herbe

1786 tó $\boldsymbol{n}$ cuillère

1787 tó $n$ nom $\diamond$ tó kpále appeler ; accuser de $(q q n-m a)$ 口 è fààn tókpàa à ma il l’a accusé de vol ; Christ. tóbhole glorifier $\square$ kذ̀ó Wàànbhaa é tóbho glorifions Dieu!

1788 tóbhóleya <tó+bhóle+ya> $\boldsymbol{n}$ gloire

1789 tobhl $\varepsilon \boldsymbol{n}$ affluent

1790 tòòmàsíé $\boldsymbol{n}$ signe, symbole (scarification)

1791 tóónle $v 1$. s'épanouir, pousser (jeunes feuilles) $\square$ án yaan gban É tóónlà mes ignames ont pris de jeunes feuilles 2. faire pousser de jeunes feuilles $\square$ pónbhé yaan é tóónla l'engrais a accéléré l'apparition des jeunes feuilles d'igname 
1792 toflà $\boldsymbol{n}$ rivière, marigot (d'irrigation) $\diamond$ toflayí mlذ̀gba rizière irriguée

1793 tóle vt puiser, verser $\square$ yò yì tólé il veut puiser de l'eau $\square$ ò yàa yì to gbóngbó yí on puisait de l'eau avec un seau en fer

1794 tómi $\boldsymbol{n}$ un certain

1795 tón $\boldsymbol{n}$ 1. aigreur $\square$ lòmliy tón ò le citron est aigre $\diamond$ tónle aigre $\square$ lòmlin tónle $\varepsilon$ un citron aigre $\diamond$ tón dole avoir durillon $\square$ ké wi kélá pwélà bhé á tan, mú gaan ma tón do chaque animal qui sort danse cette danse, jusqu'à ce qu'il aille des durillons sur les pieds 2 . Fig.. irritable, peu communicatif

1796 tòn $\boldsymbol{n}$ forge (métier) $\diamond$ tòn kpáá forge (atelier) $\square$ ý gezí tòn kpáá lâ je vais à la forge

1797 tóni $<$ Fr. tonne> $\boldsymbol{n}$ tonne

1798 tònmi $\boldsymbol{n}$ forgeron $\square$ yaò tònmi lé il n'est pas forgeron

1799 tónwóle vi péter $\square$ dèle é tón wóa ? qui a pété ?

1800 tóy, tóymu $\boldsymbol{n}$ baoulé (ethnie, langue) $\diamond$ tóymi Baoulé (représentant de cette ethnie)

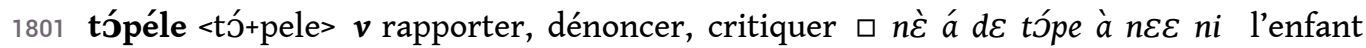
critique son père auprès de sa mère

1802 traa adj 1. plat (de vaisselle) 2. en forme d'assiette $\square$ wi mu ć kààn yì traa léc̀ bhé $\dot{\varepsilon}$ ba les animaux sont passés dans cette eau peu profonde

1803 traí: tráá $a d v$ en bas

1804 traalami rn l'aîné, le supérieur (l'enfant le plus âgé de la famille) $\square$ Zinì lè Màrìi tami lé Jules est l'aîné de Marie

1805 tráánle vi 1. rougir (prendre une teinte rougeâtre ou jaunâtre) 2. mûrir (souvent pour le riz, les mangues : pour les plantes dont les fruits changent de couleur) $\square$ blàànná mu ć tráánle ò les bananes sont mûres

1806 trala loc au-dessus $\square$ maanc̀ mu ć fé klâ à gwà trala les oiseaux ont fait leur nid sur le toit

$1807 \operatorname{tran} \boldsymbol{n}$ furoncle, abcès

1808 trèngale vi passer (toute la journée) $\square$ ó pegéé Záàn trèngàa ye líi Jean et moi, nous avons passé toute la journée au travail

1809 treprízì $<$ Fr. entreprise $>\boldsymbol{n}$ entreprise

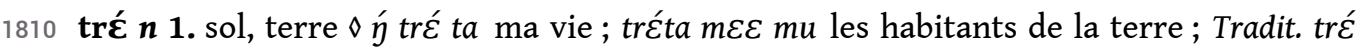
zan propiétaire de la terre 2. plancher $\diamond$ tré ma go ! balaye le plancher!

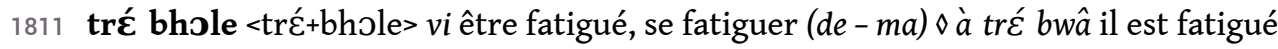

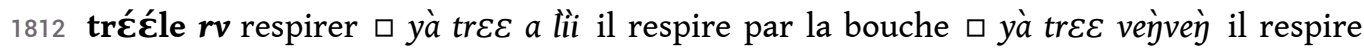
péniblement

$1813 \operatorname{tr} \grave{\varepsilon} \mathfrak{y}<$ Fr. train> $\boldsymbol{n}$ train

1814 trézan $\boldsymbol{n}$ propriétaire du terrain (le chef de la famille des fondateurs du village)

1815 trii adj petit et rond $\square$ ý tró láa triitrii mu yà j'ai vu beaucoup de petits champignons très ronds $\square$ Syn. grii

1816 trìitrìi $a d v$ ideo attentivement, en regardant autour de soi $\square$ dri zan $\varepsilon$ yò dri mu $\varepsilon^{\prime}$ taglinzí trìitrìi yì mí yre ć nój̀ le berger surveille les vaches quand elles s'abreuvent 
1817 tríy $n$ 1. $r n$ saleté $\diamond$ à tríy ò il est sale; trín dole se salir $\square$ ké $n \dot{\varepsilon}$ mu trín dwà, Zàle

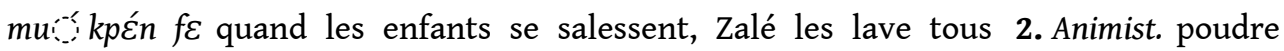
magique noire [on la met sur le front ou sur le corps contre la maladie ou les mauvais sorts] $\square \grave{e}$ tríy dwà à mlè líi il s'est mis de la poudre magique sur le front

1818 tró $\boldsymbol{n}$ champignon $\square$ le mu jà tró yróle bòn yíi les femmes sont allées ramasser des champignons en brousse $\diamond$ tróláá champignon (surtout sa partie supérieure) $\square$ zre ta tró láá

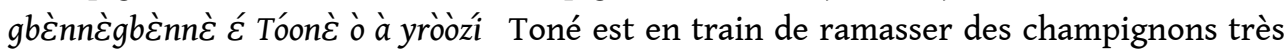
larges sur la termitière ; zù tró banc̀ termitière à étages

1819 trò $\boldsymbol{n}$ crête (d'oiseau) $\diamond$ maagwlen winta trò crête du coq

1820 trò $\boldsymbol{n}$ escargot $\diamond$ trò klo coquille d'escargot

1821 tróóle $\boldsymbol{v}$ 1) vi 1. être long, devenir long $\square$ trwâné $\varepsilon$ cíc pú mu troo les poils blancs de la panthère sont longs 2. être grand (de taille) $\square f^{\prime} \varepsilon$ tróole mu de grandes maisons $\square \grave{a}$ do troo bhéċ à do ò kpeteekpe une (fille) est grande et l'autre est petite 2) 1. vt élever 2. Fig. éduquer $\diamond$ tróómi éducateur

$1822 \operatorname{trop} \varepsilon<\operatorname{tro}+p \varepsilon>\boldsymbol{n}$ animal domestique $\square$ trope lè maa lé, trope l’e srooonc̀ lé le poulet et le chat sont des animaux domestiques

1823 tróontróongbà $\boldsymbol{n}$ papillon

1824 trón kàlá $r n$ arrière-petit fils, arrière-petite fille [ne doit pas toucher les oreilles de son arrière-grand père, on croit que quand on le fait le vieil homme meurt]

1825 tròn $\boldsymbol{n}$ bénéfice $\diamond$ tròn srój̀ wóle avoir du bénéfice

1826 tron $\boldsymbol{n}$ rhume, toux $\square$ tron ý trón kpén líi táàn ý ma le rhume m’a bouché les oreilles

1827 trón, tróón rn oreille $\diamond$ trón láá oreille, pavillon ; trónyí pe boucle d'oreille ; trón kpále écouter (à - ni à quoi - là) ; trónwimi sourd (personne)

1828 trú rn vagin $\diamond$ trú cí poil pubien (de femme)

1829 trúngóle trúngóle vi disparaître, s'enfuir, s'évader (de - kóó) ฯ fàànníi ć trún góa fáázan $m u$ ć kóó le voleur a échappé aux policiers

1830 truu adj rond $\square$ zàdomu pe tré ò truu les savants disent que la terre est ronde

1831 trúù $n$ bêtise $\square$ Màni ò trúu meE lé Mani est bête $\square$ trúù ò à ma bhîi glawò il est bête comme une hyène

1832 trwàné, trúàné n Panthera pardus léopard, panthère $\square$ Syn. srJp $\varepsilon$

1833 tùàbù <Dioula> $\boldsymbol{n}$ 1. personne de race blanche, personne blanche (y compris les Européens, Arabes, Japonais, Chinois etc.) $\diamond$ tùàbù yaan pomme de terre; tùàbù blàán banane

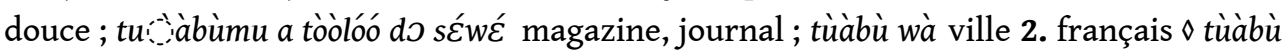

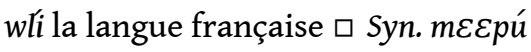

1834 túénغ̀ $\boldsymbol{n}$ orphelin

1835 tùغ̀ntùènn $\varepsilon$ n fourmi (noire, de taille moyenne, qui pique douloureusement)

1836 túytúy adv ideo considérablement (de distance en âge) $\square$ ý do ò kpén léć túytúy je suis beaucoup plus âgé qu'eux

1837 tútú $a d v$ très tôt $\diamond$ zrwanzi tútú très tôt le matin

1838 tútùle $n$ prostituée

1839 tùùbole <Dioula> vt embrasser, serrer dans les bras $\square n \varepsilon \grave{a}$ de tùùbùa l'enfant a embrassé son père 
1840 túúle vi être chiffonné, être usé (tissu, natte, toit de chaume etc.) प án fé É túúlà le toit de ma maison s'est froissé

\section{$\mathrm{U}-\mathrm{u}$, comme usa, úun, ùùwi}

\section{V - v, comme va, vaa, vaagblòó}

1844 va $n$ aigle

vaa $a d v$ désert plat (sans végétation)

vaagblòo $\boldsymbol{n}$ carpe

vàànvààn adv ideo avidement (façon de fumer)

vàn $\boldsymbol{n}$ miel (sauvage)

vàngàn $\boldsymbol{n}$ papayer $\diamond$ vàngàn kpúy papaye

vàvà $n$ zèle $\diamond$ zà vàvà dele mettre son zèle à vè̀vè̀ ideo péniblement (respirer)

véćlí <Fr. verre> $\boldsymbol{n}$ verre viande gâtée sent mauvais

vi $r n$ estomac, ventre, abdomen (animal, insecte)

viay onomatop sifflement (bruit de la balle) autre épouse) 4. l'autre objet (d'une paire)

víòn $<$ Fr. avion $>\boldsymbol{n}$ avion

vitivinè $a d v$ ideo petit et faible

vlevle, vrevre $\boldsymbol{n}$ herbe (esp.)

vàkà $a d v$ en sursaut $\square$ ý bwèlà vàkà je me suis réveillé en sursaut

vàkò $\boldsymbol{n}$ canne à sucre $\diamond$ vàkj̀ yrí canne à sucre (tiges abattues)

vamaa $\boldsymbol{n}$ Threskiornis aethiopica ibis sacré [ils accompagnent les groupes de bovins]

vè̀ $a d v$ tout de suite, aussitôt $\square \mathrm{ml \varepsilon}$ yòo lè bòn mle lé àmasròyí waa mado vè̀ le mamba vert est un serpent très dangereux parce qu'on ne le remarque pas tout de suite

vغ̀ $n$ Ceiba pentandra fromager (le plus grand arbre de l'Afrique de l'Ouest).

vèc̀le $v$ 1. diminuer, baisser 2. humilier (une personne)

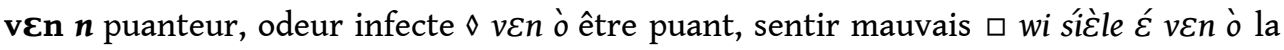

vìy adj autre, qui reste $\square m \varepsilon \varepsilon$ vìy $m u \varepsilon$ ć bwà Bíjà les autres gens sont restés à Abidjan

vìndomi $r n$ 1. voisin 2. Christ. prochain 3. coépouse (femme d'un homme par rapport à une

vléèvléè $\boldsymbol{n}$ sifflet $\diamond$ vléevlêe pénle souffler dans un sifflet

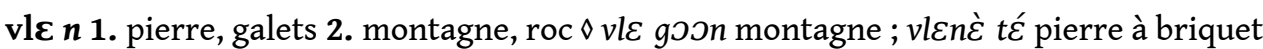

vle $n$ Cephalophus monticola céphalophe bleu, biche blanche 


\section{W - w, comme wà, wààn, wáátí}

1886 wà <ò+à> pron ils le (série contractée « sujet-objet direct »)

1887 wá <ó+à> pron nous (excl.) le (série contractée « sujet-objet direct »)

1888 wà $n$ village, ville

1889 waa <ò+ láá> pron ils ne... pas (3 pers. pl., série négative)

1890 wáá <ó+ laa> pron nous ne... pas (1 pers. pl., excl., série négative)

1891 waa <ò+a> pron leur (3 pers. pl., série possessive)

1892 wáa <ó+a> pron notre (1 pers. pl., excl., série possessive)

1893 waa diin $n$ Carapa procera okoto, touloukouna (arbre)

1894 wáá num mille $\diamond$ gòli wáá do cinq mille francs

1895 waa $\boldsymbol{r n}$ urine $\diamond$ waa wole uriner $\square$ waa laa wo g̀̀ il est interdit d'uriner ici !

1896 wààn $\boldsymbol{n}$ graine de palmier à huile (la noix qui donne de l'huile) $\diamond$ wààn yrón huile de palme ; wààn bhe une graine de palmier

1897 Waànbhaa $\boldsymbol{n}$ Christ. Dieu $\square$ áliké ó gàa oo, ó ge Waànbhaa ba même si nous mourrons, nous allons vers Dieu

wáátí <Arab>n 1. instant $\diamond$ wáátí ć kpén toujours; wáátí lá ba quand; wááti kpén ba toujours, jamais (avec la négation) $\square$ Syn. bhla

wákísì <Engl. super-wax> $\boldsymbol{n}$ tissu de coton de haute qualité, super-wax (Afr.)

wàla $<$ wà+la $>$ loc au village

wan $\boldsymbol{n}$ wan (ethnie, langue) $\diamond$ wanmi Wan (représentant de cette ethnie)

wànmì <Dioula> $\boldsymbol{n}$ beignet, galette (petite, sucrée) 
1903 wànnì $\boldsymbol{n}$ awalé (jeu) [consistant à déplacer des pions sur un plateau à 12 trous] $\diamond$ wànni bole jouer à l'awalé

1904 wànyán, wàyán $\boldsymbol{n}$ Trad. scarification (les marques sur le visage) $\diamond$ wànyán kánle faire la scarification

1905 wáò <ó+láá+ò> pron nous ne sommes pas (1 pers. pl., excl., série contractée négative avec la copule) $\square$ wáo gelè nous ne voulons pas partir

1906 wásó <Dioula> $\boldsymbol{n}$ vantardise $\diamond$ wásówóle se vanter (de - yí) $\square$ yà wásó wo yaa fé dre yíi il se vante de sa nouvelle maison

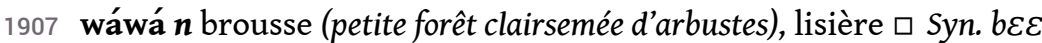

1908 wee $n$ Mallophaga pou

1909 wèè $\boldsymbol{n}$ mortier

1910 wee rn 1. queue (mammifère, oiseau, poisson, insecte) $\diamond$ bhlù wee queue de vipère 2. passé $\diamond$ $l \varepsilon \varepsilon$ wee ma l'avant-dernière année

1911 wèle vt (mob) produire un son, parler (gens), chanter (oiseaux) $\square$ delè wèí bhí lé ? qui parle de toi ? $\square$ maa gwlecn nu wèle zrwan zi le coq va chanter le matin $\diamond$ gòn $\varepsilon$ wéa la voiture s'est mise en marche ; yi-wéle résonner (de cravache); se faire une entorse $\square \dot{y}$ gaan yì-wèa je me suis fait une entorse au pied

1912 welewelematénć $\boldsymbol{n}$ petit poisson à queue rouge

1913 wén adj 1. honnête, juste, clair (d’homme) 2. Christ. saint $\diamond$ Màrìi wéy Notre-Dame, la Sainte-Vierge

1914 wésé $\boldsymbol{n}$ Ipomoea batatas patate douce, patate

1915 wewenc̀ $a d v$ ideo petits et nombreux

1916 we $n$ sel $\square$ we klá ziغ̀n $\varepsilon$ ba ajoute du sel à la sauce $\diamond w \varepsilon$ yi mer, océan

1917 weba blàán yáále $\boldsymbol{n}$ chips de banane (salé) [se préparent avec des bananes plantains non mûres] $\square$ Syn. blàa yáále

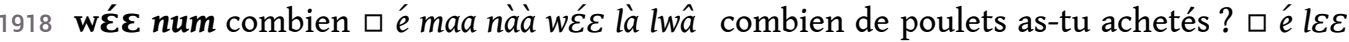
wéc lè gè ? quel âge as-tu?

1919 wغ่̀ onomat son émis par les termites

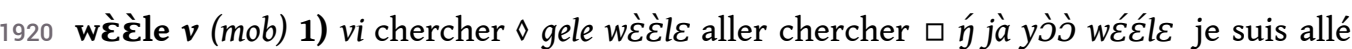
chercher du bois 2) vt Eufem. avoir des rapports sexuels (pour un homme) $\square$ Syn. yilà le ba

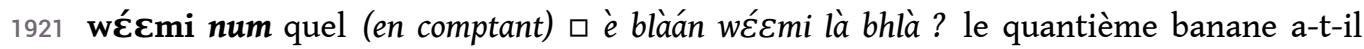
mangé ? (afr. "quelième banane...")

1922 wè̀ेn $\boldsymbol{n}$ (contr) vin de palme $\square$ wغ̇غ̀n danle ó kóó ć yáa éyóo lé le vin que nous avons goûté était mauvais $\diamond w \varepsilon \grave{\varepsilon} n d \varepsilon m i$ soûl ; wغ̇غ̀nmími buveur de vin de palme

1923 wعlغ̀ $\boldsymbol{n}$ étable (bâtiment où est logé le bétail)

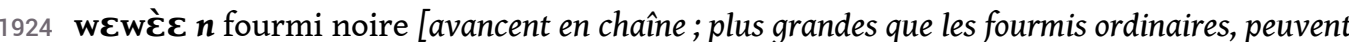
attaquer des termites]

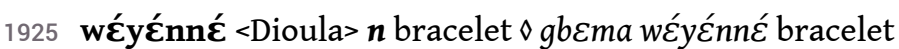

1926 wi $\boldsymbol{n}$ 1. animal $\diamond$ widemi chasseur 2. viande $\square$ trwânर́ wi wlècn bhle la panthère mange la viande crue $\diamond$ bloo wi la viande d'agouti

1927 wi $n$ pleurs 
1928 wiánle vt 1. répandre $\diamond$ yro wiánle jeter le filet; yi wiánle asperger d'eau [sur un enfant qui est malade : le guérisseur prend de l'eau dans sa bouche et asperge l'enfant] 2. semer (à la volée)

1929 wid $\boldsymbol{\varepsilon}$ doso $\boldsymbol{n}$ Megacephala, Coleoptera coléoptère (esp.)

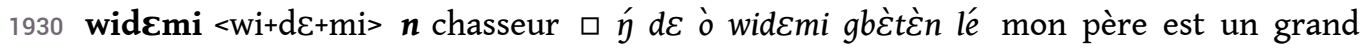
chasseur $\square$ Syn. doso

1931 wìle vt écraser, aplatir (déformer) $\square$ mángròo ć bàláá bhéċ è wìilà la mangue est tombée et écrasée

1932 wì̀n, wì̀y $\boldsymbol{n}$ 1. haut, dessus $\diamond$ wiì $m \varepsilon \varepsilon$ chef 2 . toit (d'une maison traditionnelle)

1933 wiìn adv loc (contr) 1. sur la tête $\diamond m \varepsilon \varepsilon$ wiin gale chauve (personne) 2. sur la tête (façon de transporter les objets) 3. sur la tête de $\square$ bòtò bébé ò ý wiin j'ai beaucoup de sacs sur la tête

1934 wiìy, wiin pstp (contr) sur (des objets qui ressemblent à une "tête») จ goJn ć wìi sur le sommet de la montagne

1935 wikpát ́́macienc̀ <wi+kpá+té+ma+cienc̀> $\boldsymbol{n}$ gril (pour sécher la viande)

1936 wìle $\boldsymbol{v}(m o b)$ 1) $v t$ 1. casser $\square$ yimípe $\varepsilon$ wía $n \dot{\varepsilon} m u$ ć kóó le verre a été cassé par les enfants 2. tirer avec (sur - ta) ם è màfá wía swâ ta il a tiré sur un porc 2) vi 1. se casser 2. sortir en courant (à l'encontre de qqn) $\square$ ké wà ć kpén wíle à zi et tout le village a couru vers lui

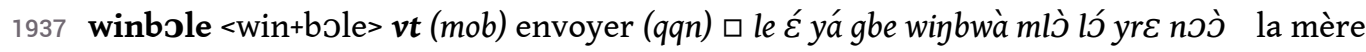
a envoyé son fils acheter du riz $\diamond$ winbowomi apôtre

1938 wiy, win $\boldsymbol{r n}$ (contr) 1. cheveux $\diamond$ win bhe un cheveu; winle cheveux $\square$ à winle ò ébèbèlé il est très chevelu; win yì-gole peigner $\square$ é wín yí go! peigne-toi !; win zi bliyle repousser qqn par la nuque

1939 wì̀gole <wìtgole> vt peigner

1940 wì̀gop $\boldsymbol{\varepsilon}<$ wìn+go+p $\varepsilon>\boldsymbol{n}$ peigne (d'un type africain) $\square$ Syn. sàkla

1941 wiyzàn <wị+zàn> $\boldsymbol{n}$ nuque $\diamond$ gele à wì̀zàn suivre qqn

1942 wisí $n$ pleurs

1943 wisíle vi pleurer, sangloter $\diamond n \varepsilon \grave{\varepsilon}$ wiśile l'enfant qui pleure $\square n \dot{\varepsilon}$ wisi a $n \varepsilon \varepsilon$ zan l'enfant pleure au dos de sa mère $\square$ ké $m \varepsilon \varepsilon$ gàà ò wisi quand quelqu'un meurt, on pleure

1944 wla $n$ couteau $\diamond$ wikáán wla couteau pour couper la carcasse d'un animal ; wla bhe lame de couteau

1945 wlàkàsà $n$ Alestes baremose péré (poisson)

1946 wlale $\boldsymbol{v}(\mathrm{mob})$ 1) vi 1. entrer, arriver $\square$ ò wà kpéé bòn ć kpén go kóókò mle mu laa wlale fála on enlève toutes les herbes du village pour que les serpents n'entrent pas dans les maisons 2. entrer (école, collège, association), se convertir (à une religion), $\square$ è wlàa baya yí il s'est converti à l'Islam 2) $v t$ faire entrer $\square$ ý gbàan wlàa fálà j'ai fait entrer le chien dans la maison $\diamond$ píké wla faire une injection; wlale à ygblo ma mettre (chapeau) $\square$ ýfáá wláa ý ýgblo ma j'ai mis un chapeau

1947 wláma <wlá+ma> loc place commune

1948 wlan $\boldsymbol{n}$ (contr) vérité $\square$ é zà wlán klâ tu as agi correctement $\diamond$ wlan kplankplan pure vérité ; $m \varepsilon \varepsilon$ wlan personne intègre ; wlan $\varepsilon$ r ma en vérité 
1949 wláy $\boldsymbol{n}$ 1. désert, Sahel 2. salon

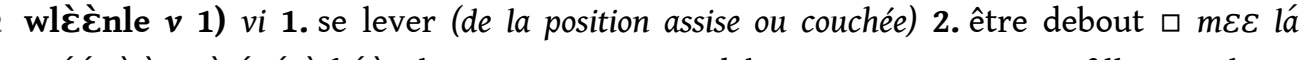
wléćnlà ò plè̀ ý lú lè kéle la personne qui est debout entre eux est ma fille 3. se lever

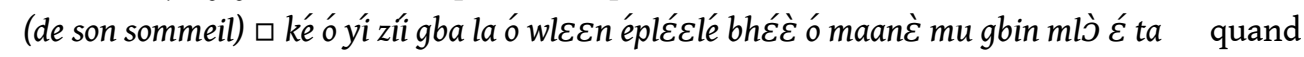
nous dormons au champ, nous nous levons tôt pour chasser les oiseaux du champ de riz 1) vt lever (une partie du corps) $\square$ kà ká gbe làa wlécn haut les mains!

1953 wlènné $\boldsymbol{n}$ chenille $\square$ ò yyỳi wlènné bhle on mange les chenilles de palmier

1954 wléwlé $\boldsymbol{n}$ pistache

1955 wleyí $n$ 1. $r n$ visage $\square$ é wleyí fé lave ton visage 2. air $\square$ à wleyí ò ké gademi lé il a l'air malade

1956 wli $\boldsymbol{r n}$ (contr) 1. son, bruit $\square \grave{a}$ gaan wli laa we ces pas ne font aucun bruit 2. langue (parole) $\diamond$ tùàbù wli ýi en français; mwàn wli la langue mwan $\square$ yóo mwàn wli pele yìdanzí j'essaie de parler la langue mwan

1957 wlin $n$ pilon

1958 wlo $\boldsymbol{n}$ boro (ancienne monnaie: bandes étroites de fer, élargies aux bouts) [auparavant, ils

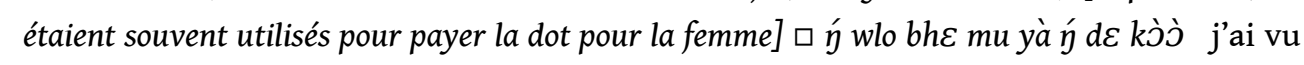
d'anciennes monnaies chez mon père

1959 wlósó < Dioula> rn obscénité $\diamond$ wlósó ma za acte obscène

1960 wlò $\boldsymbol{n}$ nasse, clôture de pêche [faite de roseaux et cannes pour pêcher dans les endroits marécageux] $\diamond$ wlò gbekpále mettre la clôture de pêche

1961 wlù $\boldsymbol{n}$ soufflet (de forge)

1962 wó pron eux (seulement dans les pronoms portemanteau)

1963 wòé <ò+é> pron ils te (série contractée « sujet-objet direct »)

1964 wólà <ò+là> pron eux, ce sont... eux que (3 pers. pl., série focalisée non-sujet)

1965 wólè <ò+lè> pron celui-là (3 pers. pl., série focalisée sujet)

1966 wole $\boldsymbol{v}($ mob) faire (verbe auxiliaire) $\diamond$ jàn wóle parler ; gba wole payer $(\grave{a}-$ ni) (pour - ma) ; frú wóle payer la dot; séli wóle prier; sríi wóle être triste; ye wole travailler; yran wole être féroce ; zran wole être jaloux

1967 wòý <ò+ý> pron ils me (série contractée « sujet-objet direct »)

1968 wóý <ò+ń> pron nous (excl.) me (série contractée « sujet-objet direct »)

1969 wóò <ó ò > pron nous (excl.) les (série contractée « sujet-objet direct »)

1970 wòò <ò+ò> pron <ò+ò> ils se, ile les (série contractée « sujet-objet direct »)

1971 wóò <ó+ò> pron nous sommes (1 pers. pl., excl., série contractée+copule)

1972 wòó <ò+ó> pron ils nous (excl.) (série contractée " sujet-objet direct »)

1973 wóó <ó+ó> pron nous (excl.) nous (excl.) (série contractée « sujet-objet direct »)

1974 wòò <ò+ò> pron wò ils sont (série contractée+copule)

1975 wóo pron ils ( 3 pers. pl., série emphatique) $\diamond$ wóomu eux

1976 wòò $\boldsymbol{n}$ singe (toutes les espèces sauf les chimpanzés)

Mandenkan, 60 | 2018 
1977 wóò bhe oo intrj au revoir ! (adieu à une personne)

1978 wò̀d $\boldsymbol{n}$ Dendroaspis mamba vert

1979 wò̀kpáán $n$ Lecaniodiscus cupanioides akika (arbre avec des fruits noirs presque pas mangéables)

\section{Y - y, comme yà, yàà, yáále}

1980 yà <è+à> pron il se, il le (série contractée « sujet-objet direct »)

1981 yá <é+a> pron ton (2 pers. sg., série possessive)

1982 yá <é+à> pron tu le (série contractée « sujet-objet direct »)

1983 yà voir ye voir

1984 yaa <è+ laa> pron il ne... pas (3 pers. sg., série négative)

1985 yaá <é+laa+ò> pron tu ne... pas (2 pers. sg., série négative avec la copule)

1986 yáá <é+laa> pron tu ne... pas (2 pers. sg., série négative)

1987 yàà <ò+à> $v$ était

1988 yàà $\boldsymbol{n}$ igname épineuse

1989 Yàà bhlé $\mathbf{m l} \boldsymbol{\varepsilon} \boldsymbol{n}$ septembre (le mois de la récolte d'igname épineuse)

1990 Yàà pá $\mathbf{m l} \boldsymbol{\varepsilon} \boldsymbol{n}$ avril (le mois où on butte les ignames épineuses)

1991 yaa pron <è+a> son (3 pers. sg., série possessive)

1992 yaà voir yalà s'asseoir

1993 yáále $\boldsymbol{n}$ >> blàán yáále banane (grillée ou frite)

1994 yààle $v \boldsymbol{t}(\mathrm{mob})$ rôtir, griller

1995 yáálí wole $<$ Dioula $>\boldsymbol{v}$ se réjouir, manifester sa joie

1996 Yaan go $\mathbf{m l} \boldsymbol{\varepsilon} \boldsymbol{n}$ février (le mois de la récolte de l'igname)

1997 yaan $n$ Dioscorea gen. igname $\diamond$ yaan pù bètèbètè (Afr. : sorte d'igname); tùàbù yaannc̀ pomme de terre

1998 yáán $\boldsymbol{n}$ savoir, connaissance

1999 yáángole $v$ connaître, savoir

2000 yàànle $\boldsymbol{v t}(\mathrm{mob})$ tailler, sculpter (bois) $\square$ yòo paan ć yáánzíi péćn ć lé il est en train de tailler l'arbre de " pan » avec un burin $\diamond$ són yáánle tailler les dents (pour l'esthétique)

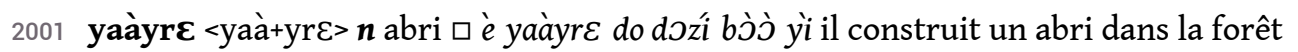

2002 yaga num trois

2003 yagami num troisième

2004 yaklà $\boldsymbol{n}$ (contr) ruse, malin

2005 yalàle, yaàle $\boldsymbol{v}$ 1) vi s'asseoir $\diamond$ yalàle à goo ba garder qqch, protéger qqn ; yalàle ò être assis $\square$ à yaàle yàa yrì kpu ta à lù lègben yre noj̀ il était assis sur le tronc d'arbre attendant sa fille 2) $v t$ 1. asseoir 2. épouser qqn $\diamond$ le yalàle épouser (pour un homme) $\square$ yóo à zi bhîi ý le yálà je veux me marier ; yalàle gwleEnna épouser (pour une femme)

2006 yale $\boldsymbol{v}$ (mob) 1. vt mettre au monde, accoucher (de qui - lé) $\square$ wòn yáà wàla je suis né au village $\square$ le $\varepsilon$ nè yàa gwláan do lé la femme a accouché d'un garçon 
2007 yàn, yáàn $\boldsymbol{n}$ 1. contenu $\diamond$ yàn do (en parlant du contenu de qch); tásá yàn do blè une cuvette d'arachide $\square$ ǵ véli yáàn do gàfé yí mîa zrwan é zi j'ai bu un verre de café le matin $\square$ à yàn do tó ý ni! sers-moi un verre! 2. coup $\diamond$ yàn do lé d'un coup $\square$ è maa dia gbe kpùy yáán do lé il a tué le poulet d'un coup de poing

2008 yànfèfè $\boldsymbol{n}$ épidermophytie, pied d'athlète

2009 yanle vi (mob) finir (de faire quoi - lé) प ý yáàn pebhlele lé j’ai fini de manger 2. vt finir $\square \grave{e}$ $f \varepsilon ́$ dole yààn il a fini de construire la maison

2010 yànna $\boldsymbol{n}$ plante (avec les feuilles amères utilisées comme l'assaisonnement au manioc, riz, igname, mil)

2011 yànnì $\boldsymbol{n}$ torche (traditionnelle) [faite d'un tronc du palmier sec]

2012 yànygán, yàyán $\boldsymbol{n}$ ananas $\square$ yàyán ć swà, kj̀ó à kán l'ananas est mûr, coupons-le

2013 yánsî́ det semblable $\square$ ý Zàle a so ć yánsi lwà j'ai acheté un pagne semblable à celui de Zalé

2014 yaò <è +laa+ò> pron il ne... pas (3 pers. sg., série contractée négative avec la copule)

2015 yáò <é+laa+ò> pron tu ne... pas (2 pers. sg., série contractée négative avec la copule)

2016 yàòbá $\boldsymbol{n}$ dan, yakouba (ethnie, langue) $\diamond$ yàobámi dan, yakouba (représentant de cette ethnie)

2017 yápe <é+à+pe> conj 1. comme $\square$ loonè pe bhle yápe vli le lièvre mange comme le rat (de la manière de manger) 2. comme si $\square$ yà kle yápe à síi láá ò il se comporte comme s'il n’y avait personne comme lui $\square$ Syn. bhîi

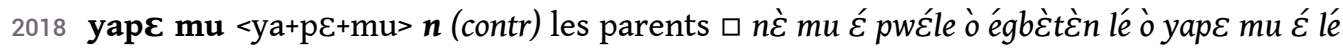
les enfants ressemblent beaucoup à leurs parents

2019 yasata adv ideo par-dessus la jambe, à la va-vite (de qqch fait négligemment ou laissé

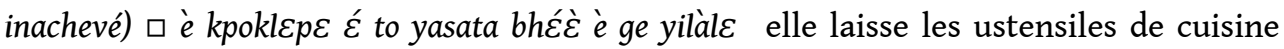
défaits et va se coucher $\square$ Syn. yabhlata

2020 ye $a d v$ là-bas (hors visibilité) $\diamond$ ó ba ye chez nous là-bas

2021 ye $\boldsymbol{n}$ travail $\diamond$ ye wole travailler

2022 yé pron lui (3 pers. sg., série contrastive focalisée)

2023 yé pron lui (seulement dans les pronoms portemanteau)

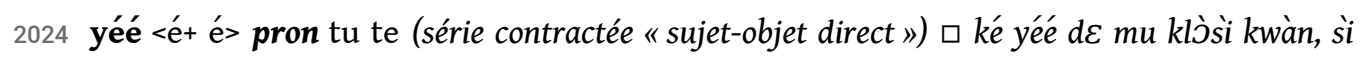
gboon ò é kóó si tu respectes tes parents, tu as une longue vie

2025 yèé <è+é> pron il te (série contractée « sujet-objet direct »)

2026 yée pron lui (3 pers. sg., série emphatique)

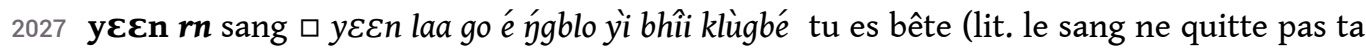

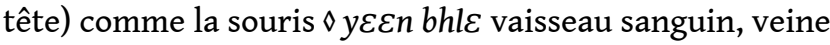

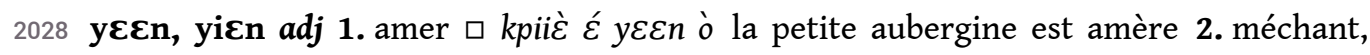

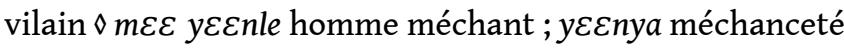

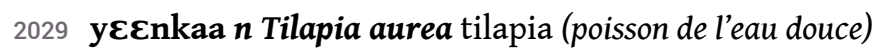

2030 yéc̀nt

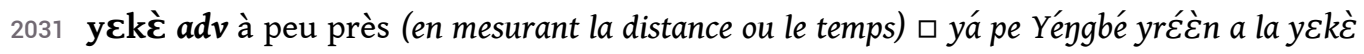
gbeغ̀n on dirait à peu près la distance d'ici au bord de Yéngbé 
yélà <è+là> pron lui, c'est... lui que (3 pers. sg., série focalisée non-sujet) $\square$ ý yéla pezí é ni gè c'est ce que je suis en train de te dire maintenant

yele $v$ 1. vt voir $\square$ klùbhòbhò yre ma ye bi zi les hiboux voient la nuit $\diamond \grave{a}$ ma fley yà je l'ai vu nu (impolitesse) ; yremayele voir clair, être clairvoyant (en sorcellerie) 2. vt considérer 口 Piéli á gbú ye élre lé Pierre se croit beau 3. trouver, décrocher, toucher $\square$ è pe è nu

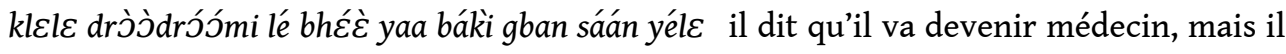
n'a pas encore obtenu son bac

2034 yélè <è+lè> pron celui-là ( 3 pers. sg., pronom sujet focalisé)

2035 yenpian $\boldsymbol{r n}$ bile

2036 yèý <è+ý> pron il me (série contractée « sujet-objet direct »)

2037 yéý <é+ý> pron tu me (série contractée " sujet-objet direct »)

2038 yéò <é+ò> pron tu es (2 pers. sg., série contractée avec la copule)

2039 yéò <é+ò> pron tu les (série contractée « sujet-objet direct »)

2040 yetanغ̀ $\boldsymbol{n}$ ouvrier agricole

2041 yewomi $<$ ye+wo+mi $>\boldsymbol{n}$ travailleur

2042 yì $\boldsymbol{n}$ (contr) 1. eau $\diamond$ yì té $\varepsilon$ eau chaude ; yi bhe goutte d'eau ; yi bhle rivière ; yi kláále faire une libation aux ancêtres $\square$ yi klálá é táa jeli mu ni, bhéč é dòj̀ bo il faut verser de l'eau à tes aïeux et tu restes en paix 2. jus $\diamond$ yàngán yí jus d'ananas; yi klele exprimer (le jus) 3. liquid $\diamond$ dùté yí thé ; zièn yí soupe ; maa yì soupe de poulet; té yí pétrole 4. sauce $\square$ pon ć lre ż̀ yí $\varepsilon^{z i}$ le foutou est bon grâce à la sauce de pangolin 5. sécrétion $\diamond$ yin yi morve ; lii yí salive

2043 yì $\boldsymbol{n}$ (contr) espace à l'intérieur (de qqch), contenu $\square$ tásá É yí ò éfley lé l'assiette est vide 口 gba yi gbaanle ò le champ est envahi par les herbes

2044 yi $\boldsymbol{n}$ jour (24 heures) $\diamond$ yi ta, yi ta bhe une fois, un jour; yi oo yi chaque jour; yinc̀ lá ta le jour où, un beau jour ; yi óó yi toujours; yi dole fixer le jour

2045 yi $\boldsymbol{n}$ sommeil

2046 ỳ̀ pstp (contr) 1. dans, en $\diamond$ gba $\varepsilon$ yí dans le champ

2047 yi vi 1. dormir 口 ó yáa yi bòn yí nous avions l'habitude de dormir dans la brousse 2. habiter, vivre $\square$ ómJว yi f́́ $\varepsilon^{\varepsilon}$ lâ nous habitons dans une maison

2048 yiàle (mob) $\boldsymbol{n}$ se porter, aller 口 é yíàa comment ça va ? kấ yíà comment ça va (chez vous)?

2049 yiàn $\boldsymbol{n}$ lombric, ver (de terre) $\square$ yiàn pwe la $\varepsilon$ ta le lombric sort sous la pluie

2050 yiàn $\boldsymbol{r n}$ oncle (frère cadet du père), tonton, petit frère du père (Afr.)

2051 yiàn yro $n$ Olax subscorpiordea santal (plante médicinale)

2052 yiba bhoorú $\boldsymbol{n}$ hippopotame

2053 yìba gbàádwà <yì+ba+gbàádwà> $\boldsymbol{n}$ Palacrocorax africanus grand cormoran

2054 yìba zu $\boldsymbol{n}$ lamproie (poisson à bouche arrondie, ayant l'apparence d'une anguille; comestible)

2055 yìbààle <yì +bààle> $r v$ faire le malin (Iv.), faire l'important $\square$ gblànغ่ $\varepsilon$, yà baa élre lé bhéc̀ yà yibaa la jeune fille s'habille bien et fait la maligne

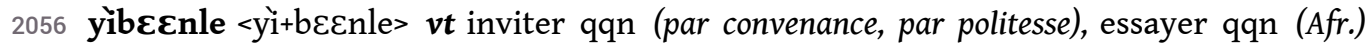
[quand on mange on ne manque pas d'inviter à manger quelqu'un qui passe; mais cette personne normalement décline l'invitation ; l'invitation n'est acceptée que si on insiste] $\square$ waa 


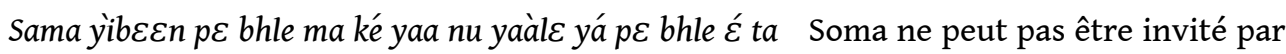
politesse, il va certainement s'asseoir pour manger

2057 yìbhole <yì+bhole> $v \boldsymbol{t}$ suffir $\square$ mlò bòtò ple ómoว yỉbho deux sacs de riz nous suffisent

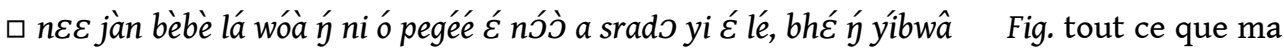
mère m'a dit le jour de notre querelle (entre mon mari et moi) m'a suffi

2058 yìbhóp $\boldsymbol{\varepsilon} \boldsymbol{n}$ Belostoma nèpe géante

2059 yìbhlole $<$ yìtbhlole $>\boldsymbol{v t} 1$. presser, serrer 2. tordre (tissu, vêtement)

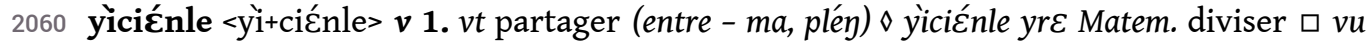
yicién yre sóó bhé kle ple dix divisé par cinq égale deux

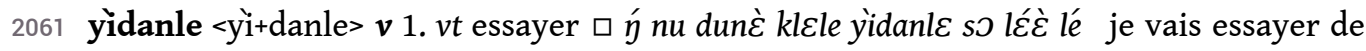
faire une chemise de ce tissu 2. tester, goûter (pour la première fois) $\square$ ǵ kókó lóa bhlele là yidanzí ż̀n aujourd'hui j'ai goûté du taro pour la première fois

2062 yìdòòle <yì+dò ̀le> vi être gentil, être doux $\nabla e$ yí kle édòj̀ lé ! sois gentil !

2063 yìdòòya $<$ yì + dòj̀+ya $>\boldsymbol{n}$ bonté

2064 yídole $<$ yi + dole $>\boldsymbol{v}$ mettre en, placer en

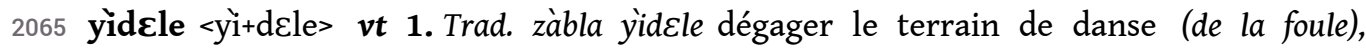
disperser les gens $\diamond$ zàbla yidemi personne qui dégage le terrain de danse 2. Fig. faire une

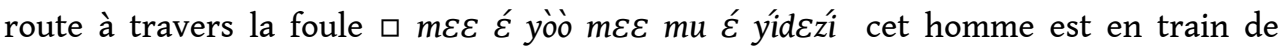
disperser les gens

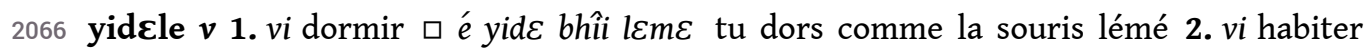
3. passer la nuit $\square$ ó yiâ sró ć tánle ta nous avons dansé toute la nuit

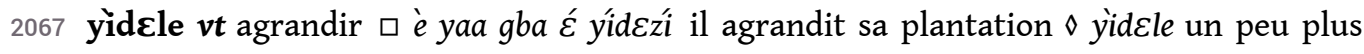
grand

2068 yid $\varepsilon \mathrm{yr} \varepsilon<\mathrm{yi}+\mathrm{d} \varepsilon+\mathrm{yr} \varepsilon>\boldsymbol{n}$ lieu à coucher

2069 yìdròàle, yìdrwààle <yì+dwrààle> vi s'apaiser $\square$ ké zà yí lrwààlà, ò pe mo kpén láá gole wà $\varepsilon$ ta si tout se calme, ils disent que les gens n'ont pas besoin de quitter le village

2070 yìdulàyr $\varepsilon<\mathrm{yì}+$ dulà+yr $\varepsilon>\boldsymbol{n}$ place pour garder de l'eau

2071 yièe $\boldsymbol{n}$ cendre [les cendres blanches sont utilisées pour couvrir le cou et le front en signe de joie]

2072 yièle $\boldsymbol{v}(\mathrm{mob}) \mathbf{1}) v t$ faire fondre $\square$ tònmi É piibhe É yie bhéć yà pii kpòo lé le forgeron fond le métal et forge la houe 2) vi fondre

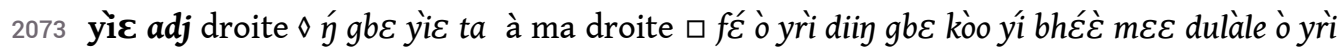
yì yí à gauche de l'arbre se trouve une maison et à droite un homme est debout

2074 yį̀ $\boldsymbol{n}$ Glossina tsé-tsé (agent d'infection de la maladie du sommeil)

2075 yícní $\boldsymbol{r n}$ ami (par opposition à l'ennemi)

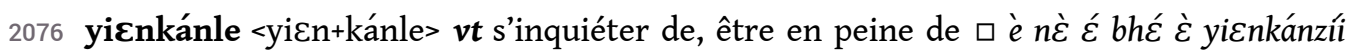
c'est l'enfant dont il s'inquiète $\square n \varepsilon \grave{\varepsilon} n \varepsilon \varepsilon$ á $n \varepsilon$ yicnkan la mère s'inquiète pour son enfant

2077 yiff́le <yì+féle> $\boldsymbol{v t}$ laver (à l'intérieur), faire un lavage, bassiner

2078 yìglàànle $<y \grave{i}+g l a ̀ a ̀ n l e>v 1 . v t$ tendre, tirer 2. $r v$ s'étirer $\square$ ké $m \varepsilon \varepsilon$ bwèla yipla, yà yìglaan quand on se sent endormi, on s'étire

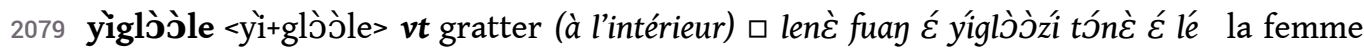
est en train de gratter dans la marmite avec la cuillère 
2080 yìglinle $\boldsymbol{v t}$ chercher le sens de qqch, savoir le sens de qqch $\square$ è jàn $\varepsilon$ yíglin dé il cherche à voir clair dans l'affaire (il essaie de trouver le sens secret de l'histoire)

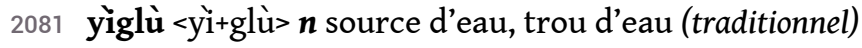

2082 yìgole $<y i ̀+g o l e>v$ 1) $v t$ 1. nettoyer à l'intérieur 2. peigner 2) $r v$ se peigner $\square$ mí $y$ wín yígo je me peigne

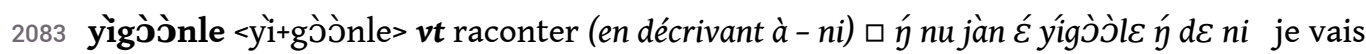
raconter cette histoire à mon père

2084 yigrònle vi ronfler

2085 yikán <yì+kán> $\boldsymbol{n}$ ruse, malice, astuce $\diamond$ yikanle rusé $\square$ Zale yikánle ò bhîi loonغ̀ Zalé est rusé comme un lapin

2086 yìkánle <yì+kánle> vi nager $\diamond$ yikánle zànta nager sur le dos

2087 yikánp $\varepsilon<\mathrm{yi}+\mathrm{ká} n+\mathrm{p} \varepsilon>\boldsymbol{n} 1$ 1. $n$ nageoire (de poisson) 2. rame

2088 yìklúúle <yì+klúúle> vi se rouler $\square$ ý mle yiklúúle yà j’ai vu un serpent enroulé $\diamond l e$ gblanc̀ dre yíklúúle la vieille femme au dos voûté

2089 yìkpále <yì +kpále> $v \boldsymbol{t}$ arracher des mauvaises herbes dans le champ (d’igname, de riz etc.) 口án mlò ć yíkpá zòn! désherbe mon champ de riz aujourd'hui !

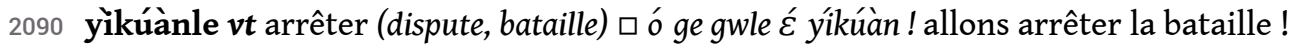

2091 yìkúnle $<\mathrm{yì}+$ kúnle $>\boldsymbol{v t}$ endurer, encaisser, supporter (recevoir une action déplaisante sans broncher) $\square$ ké twenc̀ lè é lé, é zà bèbè yíkun si tu es un orphelin, tu dois supporter beaucoup de choses $\diamond A$ yì-kún! du courage !

2092 yilablà $n$ 1. aise, soulagement (en état de paix, avoir ce dont on avait besoin depuis longtemps) $\square$ ý yilablà wòa je me suis senti à l'aise $\square$ sàyaàn mu gòa Bàànluma wà $\varepsilon$ ta, yilablà ziàn Bàànluma wà né mu é kpén ta quand les assaillant ont quitté le village, tous les gens de Bambalouma ont resenti un grand soulagement $\square$ yilablà ziàn ý ta j'ai ressenti une grande joie 2 . paix

2093 yilàle $\boldsymbol{v}$ 1. vi se coucher $\square$ yrèkpáa, è yilá lòmmli diin lii ć lâ à midi, il se couche à l'ombre de l'oranger 2. commettre adultère, coucher (avec - ba) प è yiláá dùtii ná $\varepsilon$ ba il a couché avec la femme du chef de village

2094 yilap $\varepsilon$ (yila $+p \varepsilon) n$ couchette

2095 yìmále <yì+mále> vt comprendre $\square$ náá é yíma je ne te comprends pas $\square$ tùàbù mu laa $m \varepsilon \varepsilon$ ti mu a tón yíma les Blancs ne comprennent pas les lois africaines

2096 yìmílrò <yì+mít+lrò> $\boldsymbol{n}$ soif

2097 yìmíp $\varepsilon<\mathrm{yì}+\mathrm{mí}+\mathrm{p} \varepsilon>\boldsymbol{n}$ verre (récipient)

2098 yìmláále <yì+mláále> vi 1. se gonfler 2. Fig. se gonfler d'orgueil $\square m \varepsilon \varepsilon$ É gòli gbèntèn yà, bhéc̀ yà yimláází l'homme a eu beaucoup d'argent et il se gonfle (d'orgueil)

2099 yin $\boldsymbol{r n}$ nez $\diamond$ yinlii narine ; yin yì morve

2100 Yìné $\boldsymbol{n}$ Animist. génie d'eau [est représenté comme une petite personne blanche]

2101 yinglù <yin+glù> $\boldsymbol{r n}$ narine

2102 yìniinàle <yì+niinàle> $v t$ tourner (pendant le braisage) $\square$ Zàle à bláán gon do màa bhéć yà yiníinàzí une face de la banane est cuite et Zalé est en train de la tourner sur l'autre face

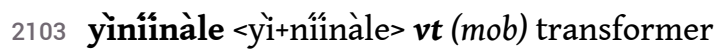


2104 yìnile $<y i$ +nile $>\boldsymbol{v}$ 1. vt tromper $\square$ bذ̀nyípe mu lè yàa kذ̀ó yíni c'étaient les génies de brousse qui nous faisaient tromper de chemin 2. vi se tromper (en faisant qqch d'autre) $\square$

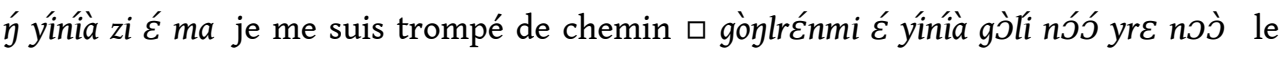
chauffeur s'est trompé de calcul

2105 yìpele <yì+pele> vt expliquer (à qui - ni) $\square$ án klegbeya ć yípía é ni je t'ai expliqué sa conduite

2106 yipla <yi+pla> adv en sommeil $\square$ ò p $\varepsilon$ ć kpén bhléa ké ý zrù ò yipla on a tout mangé pendant que ma tante dormait

2107 yìplóóle <yìplóóle> vt détacher (une corde nouée)

2108 yisata $a d v$ ideo grand et lourd

2109 yìsráále <yì+sráále> $\boldsymbol{v}$ t raccommoder (vêtement)

2110 yitakón <yìtta+kón> $\boldsymbol{n}$ pirogue

2111 yitas $\boldsymbol{\varepsilon} \varepsilon$ nì $\boldsymbol{n}$ 1. Odonata libellule, demoiselle 2. Embioptera embioptère

2112 yitréćle <yì+trććle> $\boldsymbol{v}$ se reposer $\square$ ké è yewozí, yaa yitréć vè quand il travaille, il ne s'arrête pas pour se reposer $\square$ yaa yitrćć tàmàtá míle ta il fume la pipe sans cesse

2113 yivlinle <yì+vlinle> vt distribuer $\square$ ò blúú lékpu kpánkpáy yívtilan ó ma ils nous ont distribué des morceaux de pain très durs

2114 yiwéle <yì+wéle> vt se fouler (le pied, la jambe)

2115 yiyále vt être supérieur (dans les constructions comparatives; qqn - ni) $\square$ é lrele yìya Zalé ni tu es plus jolie que Zalé

2116 yiyele <yì+yele> $\boldsymbol{v}$ 1) $v t$ savoir les pensées de qqn, voir clair dans la pensée de qqn $\square$ mí ý naagwlecn na a jàn ýíyâ, àmasròyí ý gbìy lròj̀ ò à ma je sais ce que pense la femme de mon grand frère, car elle a envie de me chasser 2) vi avoir règles $\square$ gblà mu yìye wááti $\varepsilon$ kpén ba ké mle gàà les jeunes filles ont leurs règles toutes les fins de mois

2117 yìrèèle <yì+yrèèle > vt attacher (la corde), se ceinturer (attacher la ceinture)

2118 yìzoonle <yì+zoonle> vt secouer (la racine des herbes arrachées, les tissus), remuer $\square$ kà bذ̀n $m u$ ć ýizoon la $\varepsilon^{\prime}$ lé $\varepsilon$ secouez les racines des herbes arrachées avant qu'il commence à pleuvoir (afin qu'elles ne poussent pas)

2119 yizic num quatre

2120 yiziદmi num quatrième

2121 yo $\boldsymbol{n}$ fétiche [objet, animal, végétal, minéral avec des pouvoirs surnaturels qui peuvent protéger contre la maladie, le malheur] $\square$ mùà mu yo kle mlè mu lé les Mwans font des fétiches avec des cornes d'animaux $\diamond$ yo bhe fétiche ; yoklemi féticheur qui fait les fétiches; yogbale adorer un fétiche

2122 yobhlele <yo+bhle> vi juger (en justice)

2123 yogbami <yo+gba+mi> $\boldsymbol{n}$ animiste (adepte des croyances et pratiques religieuses traditionnelles), féticheur (Afr.)

2124 yoklemi $\boldsymbol{n}$ guérisseur, charlatan (Afr.), savant [l'homme qui connaît la cause d'une maladie et quel sacrifice il faut faire pour se rétablir] $\square$ Syn. moJdomi

2125 yonغ̀ $\boldsymbol{n}$ jumeau (les jumeaux sont souvent des sorciers et sont capables de faire des choses extraordinaires; ils sont redoutables) $\diamond$ yole jumelle

2126 yòý <è+ý> pron il me (série contractée « sujet-objet direct ») 
2127 yòý $\boldsymbol{n}$ nœud coulant, boucle

2128 yòò <è+ ò> pron il les (série contractée « sujet-objet direct »)

2129 yòó <è+ó> pron il nous (excl.) (série contractée "sujet-objet direct »)

2130 yóò <é+ò> pron tu es (2 pers. sg., série contractée avec la copule)

2131 yòo $\boldsymbol{n}$ hivernage (de juillet à septembre) $\diamond$ yò bo bhla saison des pluies

2132 yòò pron il est (série contractée avec la copule) $\diamond$ yò bhîi épegée il faut, il faut que

2133 yóóyè, yóyè intrj non (réponse négative à une question)

2134 yo $\boldsymbol{n}$ palmier à huile

2135 yòo adj (contr) mauvais

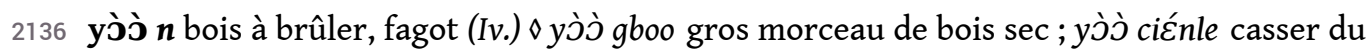
bois

2137 yoon $\boldsymbol{n}$ trace, empreinte $\diamond$ à gbe yoon empreinte de la main de qqn ; yoondcle écrire ; so yoonle pagne de couleurs traditionnelles (blanc et bleu indigo)

2138 yoondcle $v$ écrire

2139 yóónma $\boldsymbol{n}$ chameau

2140 yokop $\boldsymbol{n}$ branches de palmier (utilisées comme matériau pour la fabrication des objets)

2141 yòn $\boldsymbol{r n}$ sein, tétin, tétine (Afr.) $\diamond$ yòn líi tétin, bout de la mamelle ; yòn yíi gole traire, extraire le lait (Afr.)

2142 yòndóle <yòn+dóle> vi allaiter (des femmes) $\square$ le ć yòndóźi à nc̀ ć ni la femme allaite son enfant

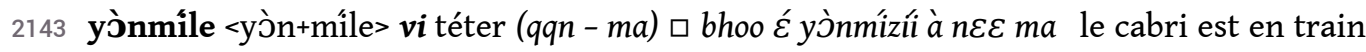
de téter les mamelles de sa mère

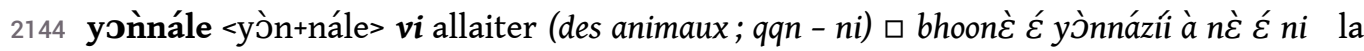
chèvre allaite son petit

2145 yota zalo $n$ Cicadellidae insecte (esp., parasite des champs de riz ; sa larve - yoyí wlènć)

2146 yowlé n yaouré (ethnie, langue) • yowlémi Yaouré (représentant de cette ethnie)

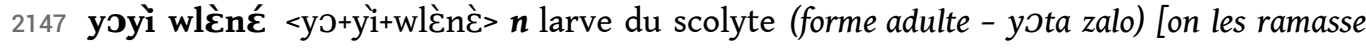
dans les palmiers abattus, et on les mange braisées ou grillées]

2148 yrà $\boldsymbol{n}$ allégresse, joie (grande joie) $\square$ à mi yrà gbú lè ò ta c'est un bonheur pour eux de (le) boire

2149 yràla $\boldsymbol{n}$ honte $\diamond$ yràla kpále avoir honte $\square$ yaa yràla kpa é léć il n'a pas honte de toi

2150 yràn $\boldsymbol{n}$ Onotragus leche, Onotragus mariae antilope (esp. : de moyenne taille, habite à côté de l'eau)

2151 yran $\boldsymbol{r n}$ colère, férocité $\diamond$ yranwole être féroce, devenir féroce

2152 yranle vi (mob) se fâcher, se mettre en colère, être en colère, flamber (feu) $\square$ ké é gbàan wee glàalà è yran é ò si tu tires la queue du chien il se met en colère $\square$ té yránzíi le feu est en train de flamboyer

2153 yranníí, nraníí $\boldsymbol{n}$ créature féroce $\square$ bhîi wi yranníi comme un animal féroce

2154 yràyrà $a d v$ ideo brillant, pétillant 
2155 yrè $\boldsymbol{n}$ lumière (de soleil), soleil $\diamond$ yromazi yrè lé après-midi (entre 16 et 17 h.) $\square$ è nwà yromazi yrè lé il est venu l'après-midi

2156 yrèèle $\boldsymbol{v t}(\mathrm{mob})$ attacher $\square$ è bhoonàa $\varepsilon$ yréézí yrì diin do $m a$ il attache la chèvre à un arbre $\diamond$ win yrèèle se tresser (ficeler des touffes de cheveux avec un fil spécial) $\square$ nu, mí é wín yrèe! viens, je vais te tresser !

2157 yrèkpáa $n$ loc (contr) midi (de 10 à 14 heures) $\diamond$ yrèkpáa do jour $\square$ è yrèkpáa yaga klà Kògòopla il a été à Kongasso pendant trois jours

2158 yrèté $n$ soleil (le contraire de l'ombre), chaleur de soleil $\diamond$ yrèté wlén bhla zi au lever du soleil ; yrèté baà bhla zi au coucher du soleil

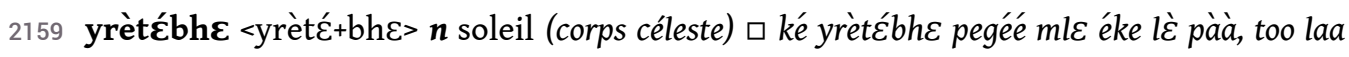
kliy quand le soleil et la lune se rencontrent, le jour ne vient pas (il y a éclipse) $\diamond$ yrètébhe púćle lever du soleil ; yrètźbhe báále coucher du soleil

2160 yrèyrè $a d v$ >> ti yrèyrè vert

2161 yrè klòàn bole, yrè klwàn bole $\boldsymbol{v}$ clignoter, cligner de l'œil $\square$ yaa yrè klwàn bwà ý ni il m'a fait un clin d'œil

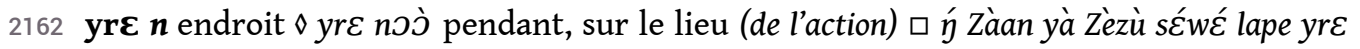
nò̀ j'ai vu Jean en train de lire la Bible $\diamond$ yre lá nój̀ la place où $\square$ án gòli é nía yre lá

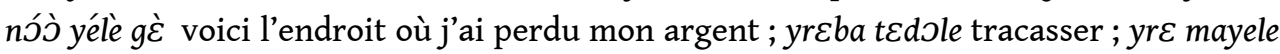
Animist. voir clair, être clairvoyant (en sorcellerie)

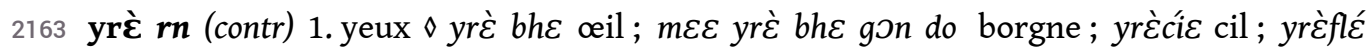
paupière ; yrèlíí paupières (lit. bouche de l'œeil) ; yrè ti pupille (d'œil) ; yrè yí larmes ; yrè ba dole regarder bien $\square$ é yré ba do yáá wi bonle lòle regarde bien, n'achète pas de la viande pourrie ; bole yrè ma vivre ; yrè tánle fermer les yeux $\square$ gbàannغ̀ mu yrèlíi tánle ò yale blaan les yeux des jeunes chiens sont fermés après leur naissance ; à yrغ̀ kóó pour plaire $\square$ án kláa é yré kóó je l'ai fait pour te plaire 2. couleur

2164 yrèbakánle adj adulte $\diamond$ meE yrèbakánle adulte

2165 yrèkpále <yrغ̀+kpále> v 1. vi regarder $(q c h-t a)$ 口 Syn. glin 2. surveiller $(q n-b a) \square e ́$ yrékpá é nè mu ba! surveille tes enfants !

2166 yrغ̀ kpámi <yrè+kpá+mi> $\boldsymbol{n}$ gardien

2167 yrema $<$ yrE+ma $>$ adj 1 . vivant 2. veillant

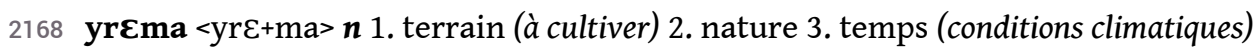

$2169 \operatorname{yr\varepsilon n} n$ 1. œuf (oiseau, poisson, reptile) 2. perle

2170 yrèn $n$ 1. sable $\diamond$ yrèn tré sable 2. souffrance $\diamond$ à yrèn klále... ma apitoyer qqn ; yrèn bhlele

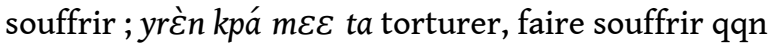

2171 yrènà $\boldsymbol{n}$ échelle [n'est jamais utilisée pour monter sur les arbres] ロ ò san yrènà ma ké wà zi bhîi ò ge fé wîin kpá on monte par l'échelle quand on veut mettre la paille sur le toit

2172 yrènblèn $\boldsymbol{n}$ amusement (petite moquerie ou plaisanterie entre des amis ou des parents) $\square$ è bhé pía yrèblèn lé il l'a dit par plaisanterie $\diamond$ yrènblèn wóle s'amuser

2173 yrèwíle $<$ yrè̀+wíle $>$ vi devenir aveugle

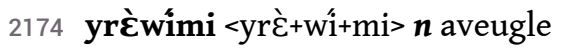

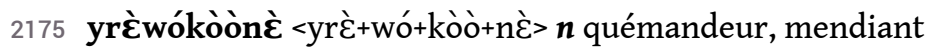




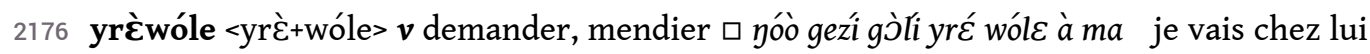
demander de l'argent

2177 yrèwómi <yrè+wó+mi> $\boldsymbol{n}$ mendiant

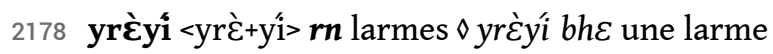

2179 yrì $\boldsymbol{n}$ (contr) 1. bois $\diamond$ yrìpómi bûcheron 2. bâton 2. bloc

2180 yri $\boldsymbol{n}$ (contr) espérances, attente, intérêt, pensée dessus (Afr.) $\diamond$ yri ò espérer (qqch - ta) $\square$ Zòzéfú da lá wóa Kànàdàa, mo kpén yrí yáà mú glaán ta quand Joseph est venu du Canada, tout le monde attendait des cadeaux

2181 yrìma kpòkpò <yrì+ma kpòkpò> $\boldsymbol{n}$ pic (oiseau)

2182 yrìma síćnú $\boldsymbol{n}$ Sahlbergella Miridae miride du cacaoyer (insecte nuisible)

2183 yrìpón koné $\boldsymbol{n}$ Anthia Carabidae carabe (esp.)

2184 yrìyáánmi <yrì+yáán+mi> $\boldsymbol{n}$ sculpteur sur bois (fait des masques, mortiers, pilons)

2185 yroma $n$ soir (de 15 à 18 heures) $\diamond$ yroma kpén zi tous les soirs

2186 yròòle $\boldsymbol{v}(\mathrm{mob}) \mathbf{1}) v t \mathbf{1}$. arracher $\square$ è yrì zikli mu ć yróóla il a déraciné les souches $\diamond$ sónbhe yröòle casser une dent à qqn., arracher une dent $\square$ Soti Sozù laaníma són bhe do yròola Soti a arraché une dent supérieure à Sozou 2. verser $\diamond$ yi yròòle verser l'eau chaude d'une marmite dans un seau pour se laver, vider une partie endiguée de la rivière (pour pêcher) 3. ramasser (prendre) [arachide, champignons qui poussent sur des termitières ou sur des arbres tombés] $\diamond$ tró yróóle ramasser des champignons; gàa yróóle couper de la paille 4. rouler, circuler $\diamond$ mí yròòà à ma bhe je m'en vais, hein !2) vi couler (de l'eau)

2187 yró̀̀ $\boldsymbol{n}$ couler (eau, rivière)

2188 yro $\boldsymbol{n}$ filet, hamac $\diamond$ kaade yro, kaadzle yro filet de pêche; niini yro toile d'araignée

2189 yrón $\boldsymbol{n}$ chaîne $\diamond$ càn yrónné chaîne d'or

2190 yròn $\boldsymbol{n}$ huile, graisse $\square \diamond$ kpén yròn graisse de biche ; zrò yròn miel ; yròn pú beurre de karité ; yròn bhe goutte d'huile

2191 yue $\boldsymbol{n}$ fleuve, lagune

\section{Z - z, comme zà, zaa, zàànweele}

2192 zà $\boldsymbol{n}$ affaire, chose $\square$ Táatóo zà do yà ze ć ýi Tato a vu quelque chose sur le palmier $\nabla$ zà blé chance, bonheur $\diamond a ̀$ zà dole être intelligent; kà sí zà ! quel dommage !; zà ké láá ò ye... il n'y avait aucune autre affaire là-bas (que...); Christ. zà yòo péché ; zà ò 1) être objet de désir (de qui - ma) $\square$ gòli bèbè zà ò Màní ma Mani veut beaucoup d'argent $\square \hat{\eta}$

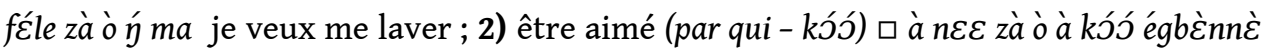
lé il aime beaucoup sa mère

2193 za $\boldsymbol{r n}$ cause, raison $\diamond d \varepsilon z a$ cause de la mort

2194 zaà conj réellement, en effet $\square$ zaà à $d \varepsilon$ lè c'est son père, donc !

2195 zaa $n$ 1. discussion, dispute, débat 2. concurrence, examen $\square$ gwláan mu É wòo zaa bJzí les hommes sont en train de se faire concurrence

2196 zaabole <zaa+bole> vi discuter (de - yí ; avec - lìi, ba) $\square$ zaa bole é ba ò églòj lé il est difficile de discuter avec toi $\diamond$ lèma zaabole contredire qqn 
2197 zaabomi <zaa+bo+mi> $\boldsymbol{n}$ discuteur, querelleur

2198 zààn n Aegoryx algazel, Gazella rufifrons, Gazella soemmeringi gazelle

2199 zàànweele $\boldsymbol{n}$ scorpion

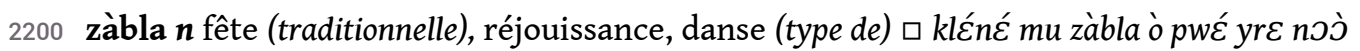
les danses en l'honneur des filles excisées ont lieu quand elles sortent (de la forêt) $\diamond$ zàblape jouet

2201 zablàle $\boldsymbol{v}$ s'amuser $\square$ ò zàblàlà ò gbú a tréta ma ils passaient leur vie en amusements

2202 zàblé $\boldsymbol{n}$ 1. bien $\square$ án zàblé láá ò tré ta je n'ai pas de bonheur sur la terre 2. cadeau $\diamond$ zàblé klele faire un cadeau (à-ni)

2203 zàdokleya $<$ zà+do+kle+ya> $\boldsymbol{n}$ ignorance

2204 zàdoleya <zà+dole+ya> $\boldsymbol{n}$ connaissance, savoir

2205 zàdomi <zà+do+mi> $\boldsymbol{n}$ divinateur, devin [fait la divination pour savoir la cause de la maladie]

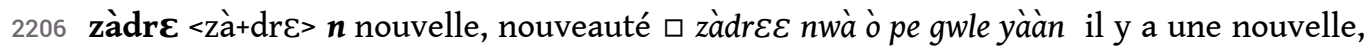
on dit que la guerre est finie

2207 zàglízàglí adv ideo zig-zag (en titubant)

2208 zàkaà $a d v$ alors, car

2209 zàké pron 1. quelque chose $\square$ zàké do kle ! fais quelque chose ! 2. rien $\square$ zàké lááò ý kóó je n'ai rien

2210 zàmáá <Dioula jàma> $\boldsymbol{n}$ foule $\square$ ý nwâ wáátí lá ba ké zàmáá sáá nwà gban ga yre ć nój quand je suis arrivé, il y avait déjà une foule de gens à la place mortuaire

2211 zan $n$ propriétaire $\square$ yaan gba $\varepsilon$ zan lè Táatóo lé le propriétaire de ce champ d'ignames est Tato $\square$ kpákò gba ć zan lè mí lé c'est moi qui suis le propriétaire de cette plantation de cocotiers

2212 zàn pstp derrière $\square$ mèlè klà bhéc̀ ká nwâ ká zàn wàla ćc̀ ? que s'est-il passé pour que vous reveniez au village?

2213 zàn $\boldsymbol{r n}$ espace derrière qqch $\diamond$ ò zan 1) revenir, s'approcher de nouveau (de - ta) $\square$ yòo zan ò ta il s'approche d'eux de nouveau $\square$ ýóo zan! j'arrive ! ; 2) commencer (faire qqch de nouveau) $\square$ bhéc̀ ké bhé ò zan banle il a commencé à pleuvoir encore

2214 záná n porte (faite de branches de palmier) [porte traditionnelle qui se lève et se baisse]

2215 Zanble $\boldsymbol{n}$ Zamblé (masque et danse de l'ethnie gouro) [se présente comme le museau d'animal]

2216 zandámù $<$ Fr. gendarme $>\boldsymbol{n}$ gendarme

2217 zànráále <zà+nráále> $\boldsymbol{v}$ s'amuser, taquiner $\square$ nè $m u$ É wòo zànráázíi gbèy $\varepsilon$ ýi les enfants s'amusent dans la cour

2218 zànta loc 1. sur le dos, au dos 2. derrière, arrière $\diamond$ f́́ $\varepsilon$ zàta derrière la maison

2219 zàntami <zànta+mi> adj dernier $\square$ ò nwà sòo é lé ká kpén zàntami là ni on a apporté le vélo à celui qui est le dernier d'entre vous

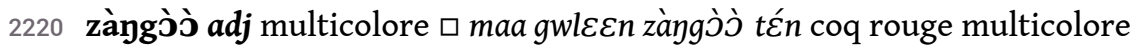

2221 zàsánle vi se quereller, se disputer $\square$ Màní pegée Zàlé zàsààn Mani et Zalé se sont querellés 
2222 zawli $n$ Zawli (masque et danse de l'ethnie gouro) [un masque avec le visage et la voix d'une belle femme porté seulement par des hommes; la jupe de fibres est très courte; la danse a un caractère divertissant et joyeux dans des événements significatifs, par exemple, aux enterrements]

2223 zayí, zaí <za+yì pstp à cause de, pour (avec le verbe) ם è yrì gbe ć kpá zíi éke ta kpàràkpàrà tán zayí il met les branches ensemble pour faire un brancard

2224 zèklè $\boldsymbol{n}$ grillon (mangeable), chenille rouge

2225 zeklee adv sans cesse, beaucoup $\square$ ý yewoà zeklee le ć zi, bhéc̀ náá le É gbú sròwóle j'ai beaucoup travaillé pour obtenir cette femme, mais je ne l'ai pas eue

2226 zèklè yùkú $\boldsymbol{n}$ chenille (couverte de poils rouges; comestible) [est mangée grillée; les poils étant préalablement enlevés au-dessus du feu]

2227 zey $\boldsymbol{r n}$ angle (extérieur) $\square$ è cienc̀ $\varepsilon$ dùláá tàbàli $\varepsilon$ zey ta il a mis le panier sur l'angle de la table

2228 zetì $n$ maître de maison, tuteur $\diamond$ zetimi maître de la maison ; zetile maîtresse de maison zèzè adj authentique, vrai $\square$ wigle zèzè lè gè c'est une chevelure vraie $\square$ mí ý náágwle zèzè yà j'ai vu mon vrai grand frère

$2230 \mathbf{z \varepsilon} \boldsymbol{n}$ raphia

2231 zEgbo $\boldsymbol{n}$ Polypterus senegalus polyptère du Sénégal (poisson) [poisson avec un corps ressemblant à un serpent]

2232 zènéyì moo $\boldsymbol{n}$ Dendrohyrax daman d'arbre

$2233 \mathbf{z} \boldsymbol{\varepsilon}$ nle $\boldsymbol{v} \mathbf{1})$ vt 1. décharger qqn (de la tête) $\square$ ý nwâle ý ziàn ma petite sœur m'a déchargé $\Delta$ yà kpó ziàn il s'est agenouillé 2. être premier (dans la course, dans le travail, dans la

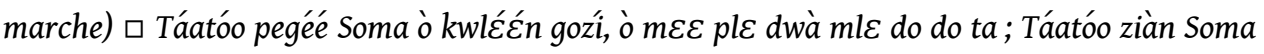
lè่ Tato et Soma récoltent le coton; chacun se met sur une ligne ; Taatoo finit avant Soma 2) vi 1. trouver porte close, manquer (qqn zànta) 2. tressaillir $\diamond$ zEnle zi entourer

$2234 \mathbf{z} \varepsilon$ nle $\boldsymbol{v}$ faire écho, retentir, résonner $\square$ à wele $\varepsilon$ z $z$ nzí fर́ ý́ fléy $\varepsilon$ ýi sa voix retentit dans une maison vide

$2235 \mathbf{z} \boldsymbol{\varepsilon} \boldsymbol{n} \boldsymbol{n}$ crinière

2236 zi $n$ sentier, chemin $\diamond$ zi gbe\& carrefour

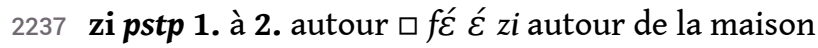

2238 zi pstp dans (un récipient, rivière) $\square$ è kaa yà Kaan $\varepsilon$ zi il a vu des poissons dans la rivière de Kaan $\diamond \grave{o}$ à zi (bhîi) vouloir

2239 zi $\boldsymbol{r n}$ fesses, derrière, partie postérieure $\square$ ǵ nu yaàle gòn $\varepsilon$ zi zàn je vais m'asseoir à l'arrière de la voiture $\diamond \dot{y}$ gele $y$ zi zi je vais déféquer

2240 zibòò rn enfant (qui est né immédiatement après un enfant qui est mort)

2241 ziboole <zi+boole> $\boldsymbol{v}$ passer sans se voir (l'un l'autre) $\square$ Mání pegéé à na zibj̀̀la Mani et sa femme sont passées sans se voir

2242 zibole $<\mathrm{zi}+\mathrm{b}$ le $>\boldsymbol{v} 1$. vt répliquer, rendre zi) $\square$ yèn zwàn wòa ébèbè lé, náá yaa jàn $\varepsilon$ zibJle il m'a beaucoup insulté, mais je n'ai pas répliqué 2 . $v t$ prendre son élan

2243 ziغ̀n $\boldsymbol{n}$ sauce $\diamond$ seyi zicn sauce graine (Iv.) ; ziغ̀n gba potager

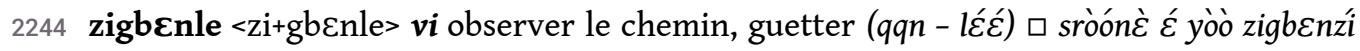
moว $m u$ lè่ le chat guette les souris 
2245 ziglìnle <zi+glinle> vt Pejor. être curieux, fourrer son nez $\square$ è za ziglin wáátí kpén ba il fourre son nez partout

2246 zigole <zi+gole> $v$ t rendre un coup $\square$ ké wò ý né $\varepsilon$ zwàn, è yaa $p \varepsilon$ zigo quand on frappe mon enfant, il rend le coup

2247 zikánle $<\mathrm{zi}+\mathrm{kay} l e>v$ 1. vi débroussailler (autour de qqch) 2. Fig. manquer (des pieds, bras; à cause de la fatigue), s'engourdir (doigts, mains) u le É gbe kpén zikààn à ma la femme a les mains engourdies

2248 zíkèlíkè $\boldsymbol{n}$ Myrmeleon fourmi-lion $\square$ zíkèlikè kle bwe yí la fourmi-lion vit dans le sable

2249 zikli $\boldsymbol{n}$ 1. $r n$ hanche 2 . souche

2250 zikpáále <zi+kpáále> vi tracer une voie (dans la brousse ou dans la forêt, pour la première fois)

2251 zikpále $<\mathrm{zi}+\mathrm{kpále}>\boldsymbol{v t}$ répliquer, renvoyer la parole, donner réponse (à la renverse) $\square$ à vìndomi á zwàn wòà, bhéć yaa bhé zikpále sa co-épouse l'a insultée, mais elle n'a pas répliqué

2252 zikùy $\boldsymbol{r n}$ fesses

2253 zilîí <zi+liî̀> $\boldsymbol{r n}$ marieur, intermédiaire (vient à la maison du père de la future fiancée) [habituellement c'est un homme, représentant d'un autre clan ou groupe ethnique; il utilise des expressions allégoriques; dans cette affaire il n'a aucun intérêt pécuniaire direct]

$2254 \mathbf{z i m i}<\mathrm{zi}+\mathrm{mi}>\boldsymbol{n}$ disciple, adèpte

2255 Zíná $n$ 1. Zina (le génie) [les danseurs de Zina s'habillent avec un tissu comportant des petits miroirs et des petites cornes d'animaux; ils se maquillent avec du kaolin; ils peuvent attraper des sorciers et prononcer des prophéties] Ziná tánle femme danseuse de magique dance pour Zina 2. diable $\diamond$ zínáné $m u$ démons

2256 zinàle $\boldsymbol{v}$ (const) 1) vi descendre (d'un arbre, d'une voiture - yí) $\diamond$ zinà à yí ! il faut y descendre ! 2) vt 1. faire descendre $\square$ zandámù ć gòolrénmi ć zináá gòo ć ý́ le gendarme a fait descendre le conducteur de la voiture 2. faire descendre, baisser $\square$ plàntii, kúé kpá

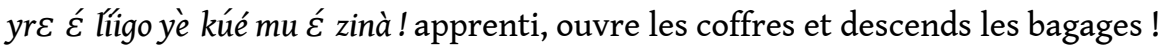

2257 zisánle <zi+sánle> $\boldsymbol{v t}$ débuter, commencer (dès le début) $\square$ ó ye é zisààn zrwan zi bhéc̀ wá léyáàn yromazi nous avons commencé le travail le matin et terminé le soir

2258 zitole $<$ zi+tole $>\boldsymbol{v}$ se venger, rendre la pareille

zitón $\boldsymbol{r n}$ aigre, tourné (aliments qui commencent à se gâter) $\square$ wèc̀n $\varepsilon$ zitón ò le vin a tourné $\diamond$ zitónle aigre

2260 ziwole <zi+wole> $\boldsymbol{v t}$ désherber, déblayer autour de qqch $\square$ bòn bhwà ébèbèlé Soma a f́é $\varepsilon$ $z i$, bhéċ è pe (káá) à gbe yaa fé ́́ zi wo beaucoup d'herbes ont poussé autour de la maison de Soma, et il dit à son fils de désherber autour de la maison

2261 zizi $\boldsymbol{n}$ besoins naturels $\diamond$ zizi kpále se soulager

2262 zo n Agama agama margouillat (lézard) [les petits Dioulas chassent et mangent les margouillats]

2263 zóó $\boldsymbol{r n}$ raison $\square$ zóó ò é zi tu as raison; zóó láá ò é zi tu as tort $\square$ zóó o dùtii zi wááti $\varepsilon$ kpén ba le chef du village a toujours raison ;é zóólè pardon, s'il te plaît; à zóólè qu'il me pardonne! 
2264 zózó $<$ Fr. ciseau> $\boldsymbol{n}$ burin, entailloir $\diamond$ plin go zózó entailloir (instrument qui sert à tailler l'intérieur d'un tambour) $\square \dot{y} d \varepsilon$ plin glù go zózó là lè c'est avec l'entailloir que mon père fait le tambour

2265 ż̀ $\boldsymbol{n}$ Manis tricuspis pangolin

2266 zo $n$ pioche $\square$ ò yJ po zJ lé on abat le palmier à huile avec une pioche

2267 zò bháliné, zò bháàné $\boldsymbol{n}$ Manis tetradactyla pangolin (à longue queue)

2268 zòkótànع n Manis tetradactyla, Manis tricuspis pangolin à longue queue, pangolin à écailles tricuspides

2269 Zòmàplé $\boldsymbol{n}$ jeudi

2270 zòn $\boldsymbol{n}$ aujourd'hui $\diamond$ ż̀n yi lè̀े $m a$ actuellement

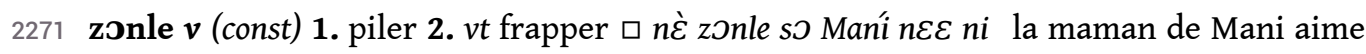
frapper les enfants $\square$ wò éke zwàn ils se sont battus

2272 zoonle $\boldsymbol{v t}$ remuer, agiter $\square$ yi póó ć yitakón é zoonzí les vagues agitent la pirogue $\square$ è magrooo gbe zoonzí kóókò à bhaa mu ć klálá il secoue une branche de manguier pour faire tomber le fruit

2273 zrá $\boldsymbol{n}$ tabac (plante, feuilles vertes)

2274 zra rn pénurie, manque $\square$ yi zra ò voJ bhla zi; ké voJ kààn, yi zra do il y a un manque d'eau pendant l'harmattan

$2275 \operatorname{zran} n$ jalousie $\diamond$ zranwole être jaloux $(d e-b a) \square$ lenc̀ $\varepsilon$ zran wozí à vìndomi ba la femme est jalouse de sa co-épouse

2276 zrazra adv ideo large, grande et pointue (feuilles des plantes, épluchures d'igname) $\square$ zrá láá $\varepsilon$ yò zrazra les feuilles de tabac sont longues et larges

$2277 \operatorname{zr} \boldsymbol{\varepsilon} \boldsymbol{n}$ 1. tas 2. termitière

2278 zro $n$ Dorylinae magnan (fourmis brunes très voraces et piquantes qui marchent en colonne; dangereuses même pour des animaux assez grands) $\diamond$ zro sáá une colonne de fourmis

2279 zro $n$ Tragelaphus scriptus, Boocercus euryceros guib harnaché, bongo (antilope de taille moyenne)

2280 zromaa $\boldsymbol{n}$ Phoenicopterus ruber flamant rose

2281 zrò bhóbhó $\boldsymbol{n}$ Bombyliida bombyle

2282 zrò $n$ abeille (collectif) $\diamond$ zrò bhe une abeille; zrò yrón miel ; zrò kpáfź ruche

2283 zròòle vi s'évanouir $\square$ srop lè̀ è è zròj̀là en voyant la panthère, elle s'est évanouie

2284 zroOnle $v \mathbf{1}) v t \mathbf{1}$. montrer $(\grave{a}-n i) \diamond$ gbe zroonle montrer du doigt $\square$ é gbe zroon a ta! montre du doigt ! 2) rv se vanter $\square$ yà gbú zron il est vantard

2285 zron $\boldsymbol{n}$ Gnathonemus tamandus poisson-éléphant

2286 zròn $\boldsymbol{n}$ haricot vert

2287 zroỳbole $n$ Hemichronis bimaculatus cichlidé à deux taches

2288 zru $n$ 1. $r n$ foie 2. Fig. cœur $\square$ Zàle zru lre le cœur de Zalé est bon $\diamond \grave{a} z r u$ ba té ò s'inquiéter ; à zru ba zinà se calmer ; zru glò̀ lé courageux ; zru kpáále être content, être heureux; zru ma le la femme aimée $\square$ Piéli á zru ma le yàláá Pierre a épousé la femme qu'il aimait ; ý zru ò é ni je compte sur toi ; zru wlale à kpéé encourager qqn

2289 zrù rn tante (sceur du père) 
2290 zruy $\boldsymbol{n}$ >> zruy gbe coussinet (tissu enroulé pour poser une charge sur la tête), bourrelet (mis sur la tête pour porter le fardeau)

2291 zruplìile <zru+plìile> vt se fâcher contre $\square$ Màní neE zruplìilà zòn Mani s'est fâché contre sa femme aujourd'hui

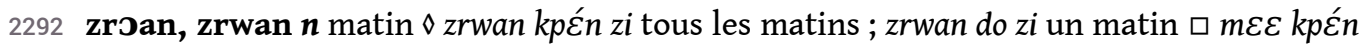
$m u ́ f \varepsilon$ zrwanzi tout le monde se lave le matin $\square$ ó nu zrwan là zi c'est le matin que nous venons

2293 zù $\boldsymbol{n}$ termitière (petite) $\diamond$ zù tróbanc̀ termitière à étages

2294 zùàngbetakánwla $<$ zuəaən+gbe+ta+kán+wla> $\boldsymbol{n}$ poisson (esp.; de rivière)

2295 zùfú <Fr. juifs $\boldsymbol{n}$ juif

2296 zùzù n Animist. génie, esprit (les puissances surnaturelles) $\diamond$ zùzù yóo mauvais esprit

2297 zùàn, zwàn $\boldsymbol{n}$ garçon, jeune homme (de 14-30 ans) $\square$ zwàn mu jà les jeunes hommes sont partis $\diamond$ zùànǹ̀ petit garçon

2298 zùàn, zwàn $\boldsymbol{n}$ injure, insulte $\diamond$ zwànwóle insulter, injurier $\square$ zwan mu ò na mu zwànwó bhla kpغ̀n ba les jeunes hommes insultent toujours leurs femmes

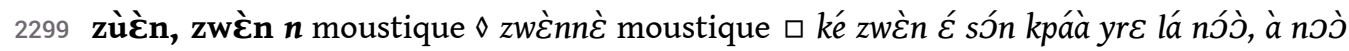
kaa là où le moustique pique, ça démange

\section{Dictionnaire des noms propres}

2300 Bāsāplā $n$ Bassapla (un des villages mwan)

2301 Bàànbòj̀ <Gouro 'forêt des singes'> $n$ Bambo (la forêt entre les villages Bambalouma et Soukourougban)

2302 Bàànluma <Gouro baan 'singe'> $n$ Bambalouma (le plus gros village mwan, nommé par le nom de la forêt - Bàànbj̀j ; clans: Yààyrí, Siágbe, Zj̀j̀gbe, Kaangbe, Yiććgbe, Gògbe, Dìgbe, Gògbe) wòn yáa Bàànluma je suis né à Bambalouma

2303 Bíjàn Bíjà <Fr. Abidjan> $n$ Abidjan $\diamond$ Bíjàn wà yíi d'Abidjan

2304 Blo $n$ Béré (rivière dans le pays mwan)

2305 Bhaakópla $n$ Bakopla (un des villages mwan; clans : Zrغ̀gbe)

2306 Bhaapla $n$ Bapla (un des villages mwan; clans : Yààyrí, Gwáà, Jiéébi) ó drúanc̀ Màní ò dùùtìi lé Bhaapla notre neveu Mani est le chef du village de Bapla

2307 Bhalèpla $n$ Balepla (un des villages mwan; clans : Yààyrí, Gògbe, Kwààngbe, Bomiso)

2308 Bhวò $n$ Boh (nom personnel, donné à l'enfant né après des jumeaux) [la personne née après des jumeaux possède un pouvoir magique extraordinaire plus que les jumeaux] Bhò̀le Bolé (femme née après des jumeaux)

2309 Caluma $n$ Tialouman (un des villages mwan ; clans : Yààyrí, Kwààngbe, Gwáà)

2310 Dalowa $n$ Daloa (ville en Côte d'Ivoire)

2311 Dàngbè $n$ Dangbé (un des clans des mwan ; forme féminine - Dànlú)

2312 Dànlú $n$ Danlu (femme du clan Dàngbè)

2313 Declú $n$ Delu (femme des clans Kwaangbè, Yegbe) [ces clans ont les mêmes tabous alimentaires : ils ne mangent pas le léopard et d'autres félins, le silure] 
2314 Digbe, Drigbe $n$ Digbé (un des clans des mwan ; forme féminine - Dilú ; groupes totémiques $m w \grave{\varepsilon}$, tàn)

2315 Dìlú $n$ Dilu (femme des clans Dìgbe, Kaangbe)

2316 Fàsàpla $n$ Fassapla (un des villages mwan; ; clans : Siágbe)

2317 Fòfúc̀ Bwàyîi $n$ Houphouet Boigny (président de la Côte d'Ivoire)

2318 Frànzi $<$ Fr. Frances $n 1$ France ò yàa Frànzi ils étaient en France 2 Français (nation) $\diamond$ fràzì $m u$ les Français

2319 Glàáyìpla $n$ Grahipla (un des villages mwan ; clans : Siágbe, Kààngbe)

2320 Glaòglùta $n$ Gblagblasso (un des villages mwan; clans : Siágbe, Zrègbē)

2321 Gògbe $n$ Gogbé (un des clans mwan ; forme féminine - Gòolú ; groupes totémiques - fì̀yj̀̀̀, yey, nrà̀, waà̄)

2322 Gòòlú $n$ Golu (femme du clan Gòogbe)

2323 Gògbe $n$ Gogbé (un des clans des mwan ; forme féminine - Gbèngba)

2324 Gòy $n$ Gon (prénom personnel d'homme) [traditionnellement donné à un enfant qui doit adorer la montagne]

2325 Goy $n$ Gông (la rivière près de Bambalouma) $\square$ Bàànluma Táátoo pégeé Po lè yàa dúléy bozí Goy ta Tato et Po de Bambalouma font la pêche à la rivière Gông

2326 Gròpla $n$ Gropla (quartier dans la sous-préfecture Kongasso)

2327 Gwâ $n$ Goua (un des clans des mwan ; forme féminine - Gwàna ; groupe totémique - bàó)

2328 Gwâna $n$ Gouana (femme du clan Gwâ)

2329 Gbàbhèlè $n$ Gbamelé (nom donné à rouquin)

2330 Gbèngba $n$ Gbengba (femme du clan Gògbe)

2331 Gbèkغ̀dù $n$ Bouaké (une grande ville de Côte d'Ivoire)

2332 Gwánà $n$ Konandidougou (un des villages mwan; clans : Siágbēe, Jàacè, Sumaro)

2333 Jiélù $n$ Jielu (femme du clan Yààri)

2334 Jię < masque Jīe > Djé (prénom des hommes)

2335 KaamJo $n$ Karamoko (un des clans des mwan; forme féminine - Kaamoว ; groupe totémique -zذ̀)

2336 Kawaa $n$ Kaoua (nom personnel d'homme)

2337 Kaangbe $n$ Kangbé (un des clans des mwan; forme féminine - Dìlú ; groupes totémiques mò̀̀n, bhooy)

2338 Klanyí $n$ Kounahiri (le village principal wan)

2339 Klغ̀ygbćta $n$ Tienigbe (un des villages mwan; clans: Yèz̀gbe, Gògbe, Dìgbe, Ġ̀gbe, Trawole, Kwààngbe, Gwáà)

2340 Klòópla $n$ Korokopla (un des villages mwan; clans: Yèc̀gbe, Yààyrí, Trawole, Kwààngbe, Gwáa)

2341 Kòngòpla $n$ Kongasso (sous-préfecture, centre administratif des mwan; clans: Y⿱亠凶̇̀g ge, Zj̀̀̀gbè, Gògbe, Siágbe, Yààyri, Zrègbe, Dìgbe, Gògbe, Taawole)

2342 Koyàa $n$ Mankono (ville au nord de la région mwan)

2343 Kwata $n$ Kouata (un des villages mwan; clans : Zigbe) 
2344 Kwangbe $n$ Kwangbé (un des clans des mwan; forme féminine - Declú ; groupes totémiques - gòla, kwé)

2345 Kwaánpla $n$ Kominapla (un des villages mwan; clans : Zj̀j̀gbe, Siágbe, Yàayrí)

2346 Lroyla, Lrooyna $n$ Soukourougban (un des villages mwan; clans: Gògbe, Siágbe, Mlègbè, Zj̀ògbe, Kwààngbē, Drigbe) Attii da Lrogla Artur vient de Soukourougban

2347 Màní $n$ Mani (nom de l'homme; celui qui porte le masque que les femmes ne doivent pas voir]

2348 Mlègbè $n$ Menegbé (un des clans des mwan; forme féminine - Mlغ̀lú)

2349 Mlèlú $n$ Mlenlu (femme de clan Mlègbè)

2350 Po $n$ Po (prénom masculin)

2351 SekÈsc̀pla $n$ Gbaziasso (un des villages mwan; clans : Zògbe, Gذ̀gbe, Gwáá, Jààjè, Sumawro)

2352 Seygè $n$ Sengé (mare dans les alentours du village de Banbalouma)

2353 Secnfla $n$ Sinfra (ville des Gouro)

2354 Siágbe $n$ Siagbé (un des clans des mwan; forme féminine - Siálú ; groupes totémiques - dày, dò̀̀n)

2355 Siálú $n$ Sialu (femme des clans Siágbe, Yààyrí)

2356 Sogbena $n$ Sogbeni (un des villages mwan; clans : Yààrí, Yèc̀gbe, Kwààngbe, Gwáa)

2357 Sùúpla $n$ Supla (un des villages mwan; son nom officiel est Bataso)

2358 Táátoo $n$ Tato (nom personnel masculin) [c'est un nom euphémistique d'origine remplaçant le vrai nom de la personne]

2359 Tángófáá $n$ Tangofa (nom propre masculin)

2360 Tògbapla $n$ Togbasso (un des villages mwan; clans : Zògbe, Gذ̀gbe, Gwáà, Jàajèc, Sumawro)

2361 Tóo $n$ Too (prénom masculin)

2362 Tóone $n$ Toné (prénom masculin)

2363 Trawole, Trawale $n$ Traoré (un des clans des Mwan; forme féminine - Zj̀òlú; groupe totémique - mعغे)

2364 TukJoma $n$ Tukoma (forêt entre Bambalouma et Bataso)

2365 Vlelà $n$ Kavakro (un des villages mwan; clans : Zògbè, Trawale)

2366 Yààyrí, Yàyrì $n$ Yayiri (un des clans des mwan; forme féminine - Siálú ; groupes totémiques $-z r o$, gùà)

2367 Yéngbé $n$ Yengbé (hydronyme)

2368 Yéćgbe, Yićgbe $n$ Yegbé (un des clans des mwan; forme féminine - Declú ; groupe totémique $-z$ ron)

2369 Yòpúgon $n$ Yopougon (quartier populaire en Abidjan)

2370 Yàámìsókolo $n$ Yamoussoukro (capitale de la Côte d'Ivoire)

2371 Zàànpla $n$ Kavagouma (un des villages mwan; clans : Digbe, Gwáa)

2372 Zenúfla $n$ Zwenula (ville dans le pays Gouro)

2373 Zèzù <Fr. Jésus> $n$ Christ. Jésus $\diamond$ Zèzù sćwé la Bible; Zèzùmi chrétien, -ienne; Zèzùya christianisme ; Zèsù Krisi Jesus Christ

2374 Zગ̀ògbè, Zòògbe $n$ Zogbé (un des clans des mwan; forme féminine - Zذ̀òlú ; groupes totémiques - gbèe, longay) [ne mangent pas de rat] 
2375 Zذ̀j̀lú $n$ Zôlou (femme du clan Zj̀gbè)

2376 ZrÈgbe $n$ Zregbé (un des clans des mwan; forme féminine - Zrèlú ; groupes totémiques - gun, lroog)

2377 Zrèlú $n$ Zrelou (femme du clan Zrègbe)

\section{RÉSUMÉS}

Cette publication est un dictionnaire bilingue (mwan-français), qui contient les informations suivantes sur les unités lexicales de la langue mwan: la forme phonologique du mot, sa signification lexicale, la partie du discours, la valence, la caractéristique stylistique et les schémas d'utilisation. Les tournures idiomatiques dans lesquelles le mot est utilisé sont données à la fin de l'entrée. Certains mots sont dotés des synonymes et des quasi-synonymes. De nombreuses entrées comportent des exemples illustratifs.

L'orthographe utilisée a été élaborée en 2009, les tons sont marqués avec des signes diacritiques. Le dictionnaire est basé sur les dialectes des villages Bambaluma et Kongasso, les données ont été collectées pendant la période de 2003 à 2018. Le dictionnaire des noms propres inclut des toponymes et des anthroponymes.

La préface au dictionnaire fournit les informations nécessaires sur la grammaire de la langue mwan.

The present publication is a bilingual dictionary (Mwan-French), which contains the following information about Mwan lexical units: the phonological form of a word, its lexical meaning, valence, stylistic characteristics and usage peculiarities. It is followed by figurative expressions with the word in question; for some words synonyms and quasi-synonyms are also given. Many entries include illustrative examples in Mwan with a French translation.

The spelling of Mwan words follows the 2009 orthography, where tones are marked with diacritics.

The dictionary is based on the varieties of the villages of Bambalouma and Kongasso, with the data collected between 2003 and 2018. A separate dictionary of personal names is included, which contains toponyms and anthroponyms.

The preface to the dictionary provides the necessary information on Mwan grammar.

ДАННАЯ ПУБЛИКАЦИЯ ПРЕДСТАВЛЯЕТ СОБОЙ ДВУЯЗЫЧНЫЙ СЛОВАРЬ (МУАН-ФРАНЦУЗСКИЙ), СОДЕРЖАЩИЙ СЛЕДУЮЩУЮ ИНФОРМАЦИЮ О ЛЕКСИЧЕСКИХ ЕДИНИЦАХ МУАН: ЗВУКОВАЯ ФОРМА СЛОВА, ЛЕКСИЧЕСКОЕ ЗНАЧЕНИЕ, ЧАСТЕРЕЧНАЯ ПРИНАДЛЕЖНОСТЬ, ВАЛЕНТНОСТЬ, СТИЛИСТИЧЕСКАЯ ХАРАКТЕРИСТИКА И ОСОБЕННОСТИ УПОТРЕБЛЕНИЯ. ДАНЫ ИДИОМАТИЧЕСКИЕ ОБОРОТЫ, В КОТОРЫХ УПОТРЕБЛЯЕТСЯ ДАННОЕ СЛОВО; ДЛЯ НЕКОТОРЫХ СЛОВ ПРИВЕДЕНЫ СИНОНИМЫ И КВАЗИСИНОНИМЫ. МНОГИЕ СЛОВАРНЫЕ СТАТЬИ ВКЛЮЧАЮТ ИЛЛЮСТРАТИВНЫЕ ПРИМЕРЫ НА ЯЗЫКЕ МУАН С ПЕРЕВОДОМ НА ФРАНЦУЗСКИЙ ЯЗЫК.

ПРИ НАПИСАНИИ СЛОВ МУАН ИСПОЛЬЗУЕТСЯ ОРФОГРАФИЯ 2009 ГОДА, ТОНЫ МАРКИРУЮТСЯ ДИАКРИТИЧЕСКИМИ ЗНАКАМИ.

В ОСНОВУ СЛОВАРЯ ПОЛОЖЕНЫ ГОВОРЫ ДЕРЕВЕНЬ БАМБАЛУМА И КОНГАССО, ДАННЫЕ ПО КОТОРЫМ СОБИРАЛИСЬ В ПЕРИОД С 2003 ПО 2018 ГОД. ОТДЕЛЬНО ДАН СЛОВАРЬ ЛИЧНЫХ ИМЕН, 
ВКЛЮЧАЮЩИЙ В СЕБЯ ТОПОНИМЫ И АНТРОПОНИМЫ.

В ПРЕДИСЛОВИИ К СЛОВАРЮ ДАЮТСЯ НЕОБХОДИМЫЕ СВЕДЕНИЯ ПО ГРАММАТИКЕ ЯЗЫКА МУАН.

\section{INDEX}

Mots-clés : lexicographie bilingue, langues mandé, mandé-sud, mwan orthographe, notation tonale

motsclesru ДВУЯЗЫЧНАЯ ЛЕКСИКОГРАФИЯ, ЯЗЫКИ МАНДЕ, ЮЖНЫЕ МАНДЕ, МУАН, ОРФОГРАФИЯ, ОБОЗНАЧЕНИЕ ТОНОВ

Keywords : bilingual lexicography, Mande languages, South Mande, Mwan, orthography, tone marking 